\title{
Modelos de sobrevivência com fração de cura e efeitos aleatórios
}

Célia Mendes Carvalho Lopes

\author{
TESE APRESENTADA \\ $\mathrm{AO}$, \\ Instituto de Matemática E EstatísticA \\ DA \\ Universidade DE SÃo Paulo \\ PARA \\ OBTENÇÃO DO TÍTULO \\ Doutor EM CIÊNCIAS \\ Área de Concentração: Estatística \\ Orientador: Prof. Dr. Heleno Bolfarine
}

São Paulo, maio de 2008 



\section{Modelos de sobrevivência com fração de cura e efeitos aleatórios}

Este exemplar corresponde à redação final da tese devidamente corrigida e defendida por Célia Mendes Carvalho Lopes

e aprovada pela Comissão Julgadora.

Banca Examinadora:

- Prof. Dr. Heleno Bolfarine (orientador) - IME-USP

- Prof. Dr. Antonio Carlos Pedroso de Lima - IME-USP

- Prof. Dr. Francisco Louzada Neto - UFSCar

- Prof. Dr. Enrico Antonio Colosimo - UFMG

- Prof. Dr. Dalton Francisco de Andrade - UFSC 

Ao Gizelton,

Aos meus pais Ceci e Plínio 


\section{Agradecimentos}

Gostaria de agradecer a todos que, direta ou indiretamente, me ajudaram durante este longo período em que fiz o doutorado. Em especial, gostaria de agradecer:

Ao Prof. Dr. Heleno Bolfarine, meu orientador, pelos ensinamentos e pela paciência.

Ao Prof. Dr. Antonio Carlos Pedroso de Lima, pelas valiosas sugestões durante meu exame de qualificação.

Ao membros da Banca Examinadora, pelas preciosas sugestões, considerações, correções e incentivos que recebi durante a defesa desta Tese.

Ao Gizelton, pelo carinho, pela compreensão, por estar ao meu lado e por todo o super apoio e incentivo na confecção deste trabalho.

Aos amigos Lane Alencar e Marcelo Rocha, pelo super apoio, amizade, ensinamentos e paciência.

Aos meus pais Ceci e Plínio, minhas irmãs, meus avós (in memorian), sogros, cunhados (valeu Ros!), sobrinhos e demais familiares, pelo apoio.

Às minhas amigas do Santa Cruz: Simone Hashimoto, Luciana Bertini, Priscila Bara e Renata Marson - pela amizade e pela torcida.

Aos amigos Solange, Alcides, Lucia, Prof. Marcel e demais colegas do Mackenzie, obrigada pela força!

Ao Prof. Dr. Plinio Simões, meu orientador de mestrado, pelo eterno apoio que nos proporciona.

Aos meus amigos imeanos, sempre vou lembrar-me de vocês com carinho.

Finalmente, agradeço a todos que, mesmo eu não tendo citado aqui nominalmente, me ajudaram com apoio, incentivo e torcida. Muito obrigada! 


\section{Resumo}

Neste trabalho são apresentados dois modelos de sobrevivência com fração de cura e efeitos aleatórios, um baseado no modelo de Chen-Ibrahim-Sinha para fração de cura e o outro, no modelo de mistura. São estudadas abordagens clássica e bayesiana. Na inferência clássica são utilizados estimadores REML. Para a bayesiana foi utilizado Metropolis-Hastings. Estudos de simulação são feitos para avaliar a acurácia das estimativas dos parâmetros e seus respectivos desvios-padrão. O uso dos modelos é ilustrado com uma análise de dados de câncer na orofaringe.

Palavras-chave: Fração de cura, efeitos aleatórios, REML, Metropolis-Hastings. 


\section{Abstract}

In this work, it is shown two survival models with long term survivors and random effects, one based on Chen-Ibrahim-Sinha model for models with surviving fraction and the other, on mixture model. We present bayesian and classical approaches. In the first one, we use Metropolis-Hastings. For the second one, we use the REML estimators. A simulation study is done to evaluate the accuracy of the applied techniques for the estimatives and their standard deviations. An example on orofaringe cancer is used to illustrate the models considered in the study.

Keywords: Surviving fraction, random effects, REML, Metropolis-Hastings. 


\section{Sumário}

Lista de Figuras $\quad$ xi

$\begin{array}{ll}\text { Introdução } & 1\end{array}$

1 Modelos de sobrevivência com fração de cura $\quad 3$

1.1 Modelo de mistura . . . . . . . . . . . . . . . . . . . . . . . 3

1.2 Modelo de Chen-Ibrahim-Sinha . . . . . . . . . . . . . . . . . . 4

1.3 Modelo de mistura com efeitos aleatórios . . . . . . . . . . . . . . . . . 6

2 Modelo de Chen-Ibrahim-Sinha com efeitos aleatórios $\quad 7$

2.1 Definindo o modelo . . . . . . . . . . . . . . . . . . . . . . . . . 7

2.2 Inferência bayesiana . . . . . . . . . . . . . . . . . . . . . 10

2.3 Análise clássica . . . . . . . . . . . . . . . . . . . . . . . . 15

3 Estudos de simulação - modelo de Chen-Ibrahim-Sinha com efeitos aleatórios 19

3.1 Características . . . . . . . . . . . . . . . . . . . . . . . . . 19

3.2 Simulação - abordagem bayesiana . . . . . . . . . . . . . . . . . 20

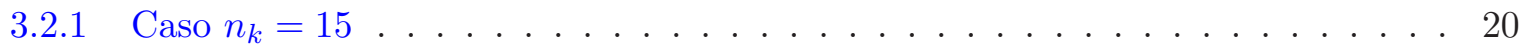

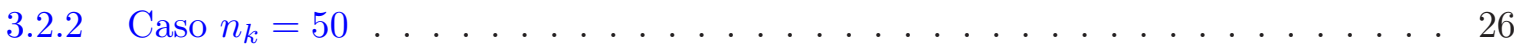

3.3 Simulação - abordagem clássica . . . . . . . . . . . . . . . . . 32

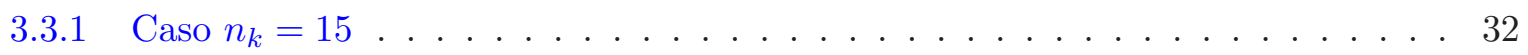

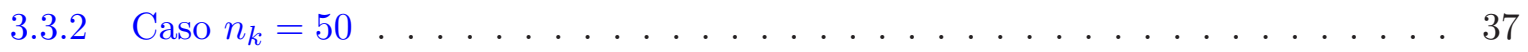

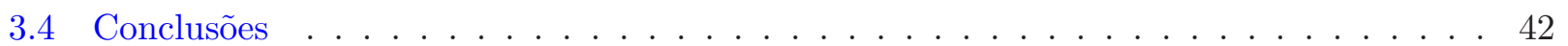

4 Análise de dados - modelo de Chen-Ibrahim-Sinha com efeitos aleatórios 45

4.1 Dados utilizados . . . . . . . . . . . . . . . . . . . . . . . 45

4.2 Inferência bayesiana via Metropolis-Hastings ～. . . . . . . . . . . . . . . . 48

4.3 Análise clássica . . . . . . . . . . . . . . . . . . . . . . . 51 


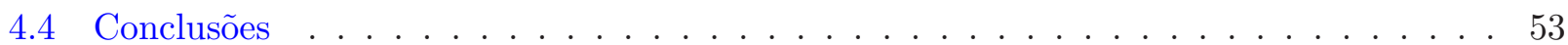

5 Modelo de mistura com efeitos aleatórios $\quad 55$

5.1 Definindo o modelo . . . . . . . . . . . . . . . . . . . . . 55

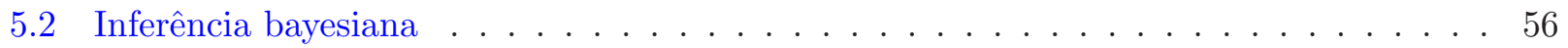

5.3 Análise clássica . . . . . . . . . . . . . . . . . . . . 58

6 Estudos de simulação - modelo de mistura com efeitos aleatórios $\quad 61$

6.1 Características . . . . . . . . . . . . . . . . . . . . . . . 61

6.2 Simulação - abordagem bayesiana . . . . . . . . . . . . . . . . . . . 62

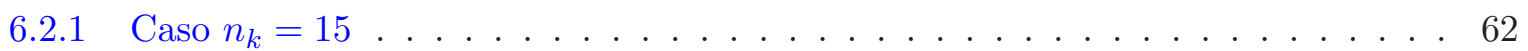

$6.2 .2 \quad$ Caso $n_{k}=50 \ldots \ldots \ldots \ldots \ldots \ldots \ldots \ldots$

6.3 Simulação - abordagem clássica . . . . . . . . . . . . . . . . . . . . . 74

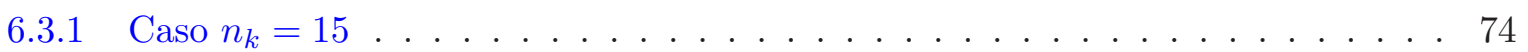

$6.3 .2 \quad$ Caso $n_{k}=50 \ldots \ldots \ldots \ldots \ldots \ldots \ldots \ldots$

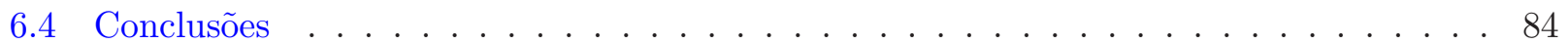

7 Análise de dados - modelo de mistura com efeitos aleatórios $\quad 87$

7.1 Inferência bayesiana via Metropolis-Hastings f . . . . . . . . . . . . . . 87

7.2 Análise clássica . . . . . . . . . . . . . . . . . . . . . . . . . . 89

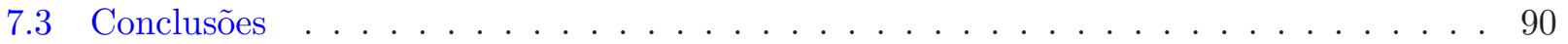

8 Conclusões $\quad 91$

8.1 Resultados e conclusões . . . . . . . . . . . . . . . . . . . . . . . . 91

8.2 Perspectivas futuras $\ldots \ldots \ldots \ldots \ldots \ldots \ldots$

$\begin{array}{ll}\text { A Programas } & 95\end{array}$

A.1 Gerando os dados para a simulação - modelo de Chen-Ibrahim-Sinha com efeitos

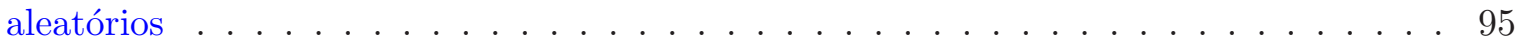

A.2 Comandos em Winbugs - modelo de Chen-Ibrahim-Sinha com efeitos aleatórios . . . . 97

A.3 Comandos em Maple - modelo de Chen-Ibrahim-Sinha com efeitos aleatórios _ . . . 98

A.4 Gerando os dados para a simulação - modelo de mistura com efeitos aleatórios . . . . 101

A.5 Comandos em Winbugs - modelo de mistura com efeitos aleatórios . . . . . . . . . 102

A.6 Comandos em Maple - modelo de mistura com efeitos aleatórios . . . . . . . . . . . . 103

$\begin{array}{ll}\text { Referências Bibliográficas } & 109\end{array}$ 


\section{Lista de Figuras}

3.1 Histogramas das estimativas dos parâmetros estimados via Metropolis-Hastings $-n_{k}=$

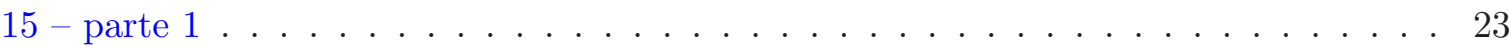

3.2 Histogramas das estimativas dos parâmetros estimados via Metropolis-Hastings $-n_{k}=$

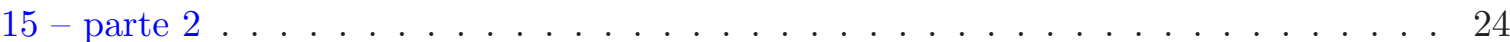

3.3 Histogramas das estimativas dos parâmetros estimados via Metropolis-Hastings $-n_{k}=$

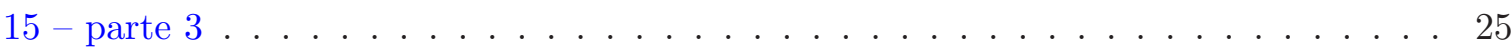

3.4 Histogramas das estimativas dos parâmetros estimados via Metropolis-Hastings $-n_{k}=$

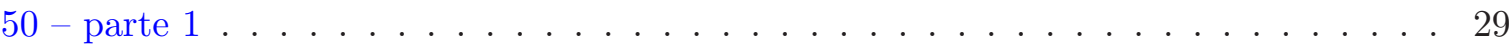

3.5 Histogramas das estimativas dos parâmetros estimados via Metropolis-Hastings $-n_{k}=$

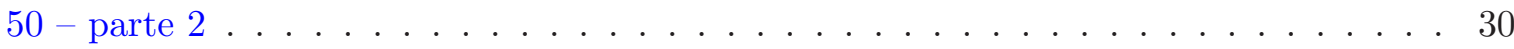

3.6 Histogramas das estimativas dos parâmetros estimados via Metropolis-Hastings $-n_{k}=$

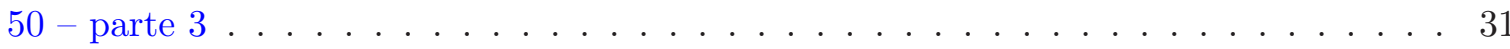

3.7 Histogramas das estimativas dos parâmetros estimados via REML $-n_{k}=15$ - parte 135

3.8 Histogramas das estimativas dos parâmetros estimados via REML $-n_{k}=15$ - parte 236

3.9 Histogramas das estimativas dos parâmetros estimados via REML $-n_{k}=15$ - parte 337

3.10 Histogramas das estimativas dos parâmetros estimados via REML $-n_{k}=50$ - parte 140

3.11 Histogramas das estimativas dos parâmetros estimados via REML $-n_{k}=50$ - parte 241

3.12 Histogramas das estimativas dos parâmetros estimados via REML $-n_{k}=50$ - parte 342

4.1 Gráficos de Kaplan-Meier para cada uma das seis clínicas . . . . . . . . . . . . . . 48

4.2 Histogramas - câncer na orofaringe - modelo de Chen-Ibrahim-Sinha com efeitos aleatórios - Metropolis-Hastings ． . . . . . . . . . . . . . . 49

4.3 Histogramas - câncer na orofaringe - modelo de Chen-Ibrahim-Sinha com efeitos aleatórios - Metropolis-Hastings - com alfa incluído . . . . . . . . . . . 50

$4.4 \sqrt{\hat{R}}$ - câncer na orofaringe - modelo de Chen-Ibrahim-Sinha com efeitos aleatórios Metropolis-Hastings . . . . . . . . . . . . . . . . . . . . . . 51

6.1 Histogramas das estimativas dos parâmetros estimados via Metropolis-Hastings $-n_{k}=$ 15 - modelo de mistura - parte $1 \ldots \ldots \ldots$. . . . . . . . . . . 65 
6.2 Histogramas das estimativas dos parâmetros estimados via Metropolis-Hastings $-n_{k}=$ 15 - modelo de mistura- parte 2 . . . . . . . . . . . . . . . . . . . . . 66

6.3 Histogramas das estimativas dos parâmetros estimados via Metropolis-Hastings $-n_{k}=$

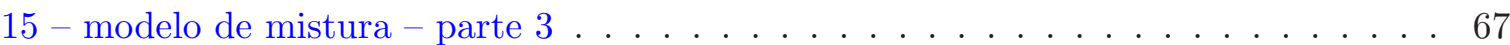

6.4 Histogramas das estimativas dos parâmetros estimados via Metropolis-Hastings $-n_{k}=$ 50 - modelo de mistura - parte 1 . . . . . . . . . . . . . . . . . . . 71

6.5 Histogramas das estimativas dos parâmetros estimados via Metropolis-Hastings $-n_{k}=$

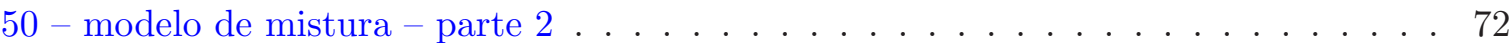

6.6 Histogramas das estimativas dos parâmetros estimados via Metropolis-Hastings $-n_{k}=$ 50 - modelo de mistura - parte 3 ....................... . 73

6.7 Histogramas das estimativas dos parâmetros estimados via REML $-n_{k}=15$ - modelo

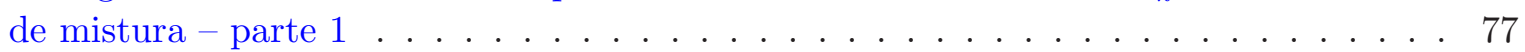

6.8 Histogramas das estimativas dos parâmetros estimados via REML $-n_{k}=15$ - modelo

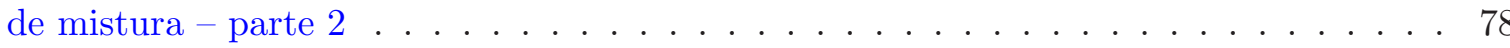

6.9 Histogramas das estimativas dos parâmetros estimados via REML $-n_{k}=15$ - modelo de mistura - parte 3 . . . . . . . . . . . . . . . . . . 79

6.10 Histogramas das estimativas dos parâmetros estimados via REML $-n_{k}=50$ - modelo de mistura - parte 1 . . . . . . . . . . . . . . . . . 82

6.11 Histogramas das estimativas dos parâmetros estimados via REML $-n_{k}=50$ - modelo

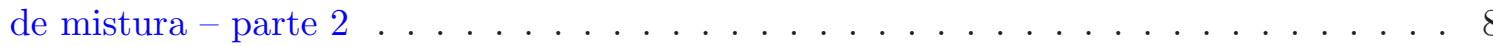

6.12 Histogramas das estimativas dos parâmetros estimados via REML $-n_{k}=50-$ modelo de mistura - parte 3 ............................

7.1 Histogramas - câncer na orofaringe - modelo de mistura com efeitos aleatórios Metropolis-Hastings .............................

$7.2 \sqrt{\hat{R}}-$ câncer na orofaringe - modelo de mistura com efeitos aleatórios - MetropolisHastings ................................. 


\section{Introdução}

Em estudos de análise de sobrevivência, avalia-se o tempo até que certo evento ocorra. Entretanto, existem casos em que parte dos indivíduos pode não apresentar o evento. Por exemplo, se um grupo de pacientes que passou por um tratamento de um mesmo tipo de câncer é acompanhado para se conhecer o tempo até o reaparecimento da doença, uma parcela deles poderá ter recidivas e outra não apresentar a recorrência do evento. Por isso o interesse em se estudar a análise de sobrevivência com fração de cura, que inclui a presença de imunes no estudo.

Muitas vezes, ao se analisar os dados de sobrevivência, percebe-se que há um grande número de indivíduos censurados à direita. Esse fato pode ser uma indicação de que, em parte dos indivíduos em estudo, o evento de interesse nunca acontecerá, ou seja, pode se ter uma fração da população que é imune (ou que se cura).

Muitos pesquisadores vêm estudando modelos de sobrevivência com fração de cura.

Em 1952, os pesquisadores J. Berkson e R. Gage publicaram um artigo (Berkson; Gage, 1952) em que apresentam um modelo de mistura para modelar os casos em que há uma proporção $p$ de indivíduos curados. Esse modelo será apresentado na seção 1.1.

Em 1999, foi publicado, por M.-H. Chen, J. G. Ibrahim e D. Sinha, um artigo (Chen et al., 1999) em que os modelos de sobrevivência com fração de cura são modelados baseado-se no número não observado de células susceptíveis a ter câncer que o indivíduo possui. Neste artigo, são abordadas algumas diferenças em relação ao modelo de mistura. Esse modelo, apresentado na seção 1.2 será denominado modelo de Chen-Ibrahim-Sinha.

Em muitos casos de análise de sobrevivência, os dados do estudo são provenientes de alguns grupos específicos, por exemplo, de várias clínicas, sendo que as respostas dos indivíduos em uma mesma clínica podem estar correlacionadas. Nesses casos, são adequados estudos via modelos com efeitos aleatórios, de modo que se possa avaliar o efeito da clínica no tratamento, por exemplo, do câncer.

Em 2001, foi publicado um trabalho de K. K. W. Yau e A. S. K. Ng (Yau; Ng, 2001) em que o modelo de mistura é analisado acrescentando-se efeitos aleatórios que pudessem medir o efeito do tratamento feito pelas diferentes clínicas nos pacientes que se curam e nos que não se curam. Tal modelo será relatado na seção 1.3 . 
Neste trabalho, é proposto o uso de efeitos aleatórios no modelo de Chen-Ibrahim-Sinha. O estudo é feito com enfoques bayesiano e clássico. Também é apresentado um estudo de simulação para ambas as abordagens. Para ilustrar a aplicação do modelo, é apresentada uma análise com os dados de Kalbfleisch e Prentice (1980, pp. 225-229), o mesmo conjunto de dados estudados no artigo de Yau e Ng (2001).

Também será analisado o modelo de mistura com efeitos aleatórios atribuídos somente à parcela de curados. Este segundo modelo será analisado com abordagens bayesiana e clássica. Do mesmo modo que para o modelo de Chen-Ibrahim-Sinha com efeitos aleatórios, será apresentado um estudo de simulação e uma aplicação a dados reais para ilustrar o uso do modelo. 


\section{Capítulo 1}

\section{Modelos de sobrevivência com fração de cura}

Neste capítulo são apresentados modelos já existentes na literatura. Primeiro é apresentado o modelo de mistura (Berkson; Gage, 1952) para estudos de casos em que há presença de imunes (ou com fração de cura). Em seguida, tem-se o modelo de Chen-Ibrahim-Sinha (Chen et al., 1999), com uma abordagem alternativa para os casos com presença de imunes. Para finalizar, é apresentado o modelo de mistura com efeitos aleatórios para curados e para não curados (Yau; Ng, 2001).

\section{$1.1 \quad$ Modelo de mistura}

Berkson e Gage (1952) propõem um modelo de mistura para os casos em que uma parte da população apresenta a possibilidade de ser curada. Este modelo também é descrito de modo sucinto em Yau e Ng (2001).

Suponha que há uma fração $p$ de curados na população e que os restantes $\pi=1-p$ são não curados. Seja $T$ o tempo de ocorrência do evento (falha). Suponha que $T=\infty$ para os indivíduos curados, de modo que a correspondente função de sobrevivência condicional é 1, para todos os valores finitos de tempo, ou seja, se $S_{1}$ é a função de sobrevivência para o grupo de curados, tem-se que $S_{1}(t)=1, \forall t \geq 0$.

Suponha ainda que a função de sobrevivência da população inteira seja dada por $S(t)$ e dos não curados seja dada por $S_{2}(t)$. Assim,

$$
\begin{aligned}
S(t) & =p S_{1}(t)+(1-p) S_{2}(t) \\
& =1-\pi+\pi S_{2}(t) .
\end{aligned}
$$

Defina uma variável indicadora de não-curados por $Y=0$, se o indivíduo for, eventualmente, curado e $Y=1$, caso contrário. Considere, ainda, o vetor de covariáveis $\mathbf{x}_{j}$ para o indivíduo $j$. Assim, a proporção $\pi$ de não curados pode ser modelada por uma função logística em $\mathbf{x}$, de modo que

$$
\pi\left(\mathbf{x}_{j}\right)=P\left(Y=1 \mid \mathbf{x}_{j}\right)=\frac{1}{1+e^{\mathbf{w}_{j}^{\top} \boldsymbol{\gamma}}}
$$


em que $\mathbf{w}_{j}=\left[1 \mathbf{x}_{j}^{\top}\right]^{\top}$ e $\boldsymbol{\gamma}$ é um vetor de parâmetros.

Se assumirmos que a função de risco condicional de $T$ para o grupo de não curados, $h_{2}(t ; \mathbf{x})$, segue o modelo de riscos proporcionais de Cox (Cox, 1972) então

$$
h_{2}(t ; \mathbf{x})=h_{20}(t) \cdot e^{\mathbf{x}^{\top} \boldsymbol{\beta}}
$$

em que $\boldsymbol{\beta}$ é o vetor de parâmetros da regressão e $h_{20}(t)$ é a função de riscos basal para o grupo de não curados. Assim, a função de risco não condicional de $T$ é dada por

$$
h(t ; \mathbf{x})=\pi(\mathbf{x}) h_{20}(t) e^{\mathbf{x}^{\top} \boldsymbol{\beta}} \frac{S_{20}(t)^{\exp \left(\mathbf{x}^{\top} \boldsymbol{\beta}\right)}}{S(t ; \mathbf{x})}
$$

Neste caso, abordagens paramétricas e semi-paramétricas podem ser adotadas. No caso de uma abordagem paramétrica, a função de risco basal é modelada parametricamente, por exemplo, seguindo uma distribuição Weibull ou log-normal.

Se considerarmos $\nu_{j}$ o indicador de falha para a $j$-ésima observação, e se $N$ é o número total de observações então a verossimilhança é dada por

$$
\begin{aligned}
L & =\prod_{j=1}^{N} f\left(t_{j} ; \mathbf{x}_{j}\right)^{\nu_{j}} S\left(t_{j} ; \mathbf{x}_{j}\right)^{1-\nu_{j}} \\
& =\prod_{j=1}^{N} S\left(t_{j} ; \mathbf{x}_{j}\right)^{1-\nu_{j}}\left(h\left(t_{j} ; \mathbf{x}_{j}\right) S\left(t_{j} ; \mathbf{x}_{j}\right)\right)^{\nu_{j}} \\
& =\prod_{j=1}^{N} S\left(t_{j} ; \mathbf{x}_{j}\right)^{1-\nu_{j}}\left(\pi(\mathbf{x}) h_{20}(t) e^{\mathbf{x}^{\top} \boldsymbol{\beta}} S_{20}(t)^{\exp \left(\mathbf{x}^{\top} \boldsymbol{\beta}\right)}\right)^{\nu_{j}}
\end{aligned}
$$

de modo que a log-verossimilhança é dada por

$$
l=\sum_{j=1}^{N}\left\{\left(1-\nu_{j}\right) \ln \left(S\left(t_{j} ; \mathbf{x}_{j}\right)\right)+\nu_{j} \ln \left(\pi(\mathbf{x}) h_{20}(t) e^{\mathbf{x}^{\top} \boldsymbol{\beta}} S_{20}(t)^{\exp \left(\mathbf{x}^{\top} \boldsymbol{\beta}\right)}\right)\right\}
$$

\subsection{Modelo de Chen-Ibrahim-Sinha}

O modelo proposto em Chen et al. (1999) é definido do seguinte modo: seja $N$ o número de células susceptíveis ao aparecimento de câncer (chamadas células carcinogênicas). Suponha que $N$ tem distribuição Poisson com média $\theta$. Seja $Z_{c}$ a variável aleatória para o tempo até que a $c$-ésima célula carcinogênica produza um câncer detectável. Supõe-se que as variáveis $Z_{c}, c=1,2, \ldots$ são iid com função de distribuição $F(t)=1-S(t)$ e também são independentes de $N$. Assim, o tempo para o aparecimento do câncer pode ser definido pela variável aleatória $T=\min \left\{Z_{c}, 0 \leq c \leq N\right\}$ em que $P\left(Z_{0}=\infty\right)=1$ e $N$ é independente da seqüência $Z_{1}, Z_{2}, \ldots$ Desse modo, a função de sobrevivência 
de $T$ é dada por

$$
\begin{aligned}
S_{p}(t) & =P(T>t) \\
& =\sum_{k=0}^{\infty} P\left(\min \left\{Z_{1}, Z_{2}, \ldots, Z_{k}\right\}>t\right) \cdot P(N=k)= \\
& =P(N=0)+\sum_{k=1}^{\infty} P\left(Z_{1}>t, Z_{2}>t, \ldots, Z_{k}>t\right) \cdot P(N=k)= \\
& =P(N=0)+\sum_{k=1}^{\infty} P\left(Z_{1}>t\right) P\left(Z_{2}>t\right) \cdots P\left(Z_{k}>t\right) \cdot P(N=k)= \\
& =e^{-\theta}+\sum_{k=1}^{\infty} S(t)^{k} \frac{e^{-\theta} \theta^{k}}{k !}=e^{-\theta}\left[1+\sum_{k=1}^{\infty} \frac{(S(t) \theta)^{k}}{k !}\right]= \\
& =e^{-\theta}\left[\sum_{k=0}^{\infty} \frac{(\theta S(t))^{k}}{k !}\right]=e^{-\theta} e^{\theta S(t)}=e^{-\theta+\theta S(t)}= \\
& =e^{-\theta(1-S(t))}=e^{-\theta F(t)}
\end{aligned}
$$

Note que, portanto, a fração de cura é calculada por

$$
S_{p}(\infty)=\lim _{t \rightarrow \infty} S_{p}(t)=\lim _{t \rightarrow \infty} e^{-\theta F(t)}=e^{-\theta}
$$

Para o indivíduo $j, \operatorname{com} j=1,2, \ldots, n$, considere $y_{j}=\min \left\{T_{j}, C_{j}\right\}$ em que $T_{j}=\min \left\{Z_{j 0}, Z_{j 1}, \ldots, Z_{j N_{j}}\right\}$, sendo $N_{j} \sim \operatorname{Poisson}\left(\theta_{j}\right)$, e $C_{j}$ a variável de censura à direita. Considere também que as censuras não informativas $C_{j}$ são independentes. Considere, ainda, a função indicadora de falha $\nu_{j}$. Para $N_{j}=n_{j}$, Chen et al. (1999) e Mizoi (2004) mostram que

$$
f\left(y_{j}, \nu_{j} \mid n_{j}\right)=S\left(y_{j}\right)^{n_{j}-\nu_{j}}\left[n_{j} f\left(y_{j}\right)\right]^{\nu_{j}}
$$

Para modelar $\theta_{j}$, podemos utilizar covariáveis $\mathbf{x}_{j}$. Como $\theta_{j}$ é positivo, pode utilizar, por exemplo, $\theta_{j}=e^{\mathbf{w}_{j}^{\top} \boldsymbol{\gamma}}$.

Além disso, pode-se especificar um modelo paramétrico para o tempo de sobrevivência. Nesse caso, tem-se que $f\left(y_{j}\right)=f\left(y_{j} \mid \boldsymbol{\lambda}_{j}\right)$. Muitas vezes, segundo Chen et al. (1999), a distribuição escolhida é Weibull ou Gama. Note que nesses casos, $\boldsymbol{\lambda}_{j}=(\lambda, \alpha)$.

A distribuição Weibull utilizada em Chen at al (1999) tem função densidade dada por $f(y)=$ $\alpha y^{\alpha-1} \exp \left\{\lambda-y^{\alpha} \exp (\lambda)\right\}$, de modo que $\alpha>0$ e $\lambda \in R$.

Para modelar $\lambda_{j}$, podemos utilizar covariáveis $\mathbf{x}_{j}$. Por exemplo, $\lambda_{j}=\mathbf{x}_{j}^{\top} \boldsymbol{\beta}$. 


\subsection{Modelo de mistura com efeitos aleatórios}

Yau e Ng (2001) apresentam uma forma de se trabalhar o modelo de mistura com efeitos aleatórios, que muitas vezes são necessários em estudos quando um as respostas dos indivíduos em uma mesma clínica podem estar correlacionadas e um dos interesses do estudo é avaliar a diferença de tratamento entre clínicas diferentes.

as respostas dos indivíduos em uma mesma clínica podem estar correlacionadas

Yau e Ng (2001) consideram o modelo de mistura com fração de cura dada por $1-\pi$ :

$$
S(t ; \mathbf{x})=1-\pi(\mathbf{x})+\pi(\mathbf{x}) S_{2}(t ; \mathbf{x}),
$$

em que $S_{2}$ é a função de sobrevivência da parcela da população que não se cura e $\mathbf{x}$ são covariáveis.

Nesse caso, $\pi$ é estimado via função logística do seguinte modo. Suponha que há $M$ clínicas e que $T_{j k}$ é tempo observado de falha/censura para o $j$-ésimo indivíduo da $k$-ésima clínica. Seja ainda $n_{k}$ o número de observações na $k$-ésima clínica e $N=\sum_{k=1}^{M} n_{k}$ o número total de observações. Então,

$$
\pi\left(\mathbf{x}_{j k}\right)=\frac{1}{1+e^{\xi_{j k}}}
$$

com

$$
\xi_{j k}=\mathbf{w}_{j k}^{\top} \gamma+V_{k}
$$

para $k=1,2, \ldots, M ; j=1,2, \ldots, n_{k} ; \mathbf{w}_{j k}=\left[\begin{array}{ll}1 & \mathbf{x}_{j k}^{\top}\end{array}\right]^{\top}$ e $V_{k}$ é o efeito aleatório correspondente à $k$-ésima clínica e que está relacionado com a fração de cura. É suposto que $V_{k}, k=1, \ldots, M$ são iid $N\left(0, \theta_{1}\right)$.

Em relação ao modelo de riscos proporcionais de Cox, em que $h_{2}(t ; \mathbf{x})$ é a função de riscos proporcionais de $T$, e $h_{20}$ é a função de risco basal para o grupo de não curados, tem-se

$$
h_{2}\left(t_{j k} ; \mathbf{x}_{j k}\right)=h_{20}\left(t_{j k}\right) e^{\eta_{j k}}
$$

com

$$
\eta_{j k}=\mathbf{x}_{j k}^{\top} \beta+U_{k}
$$

para $k=1,2, \ldots, M ; j=1,2, \ldots, n_{k} ;$ e $U_{k}$ é a variável não observável correspondente ao efeito aleatório da $k$-ésima clínica, que determina o risco de formação de tumor para indivíduos não curados. É suposto que $U_{k}$ são iid $\mathrm{N}\left(0, \theta_{2}\right)$.

Considerando $\mathbf{V}=\left[V_{1}, V_{2}, \ldots, V_{M}\right]$ e $\mathbf{U}=\left[U_{1}, U_{2}, \ldots, U_{M}\right]$, o estimador para $(\boldsymbol{\gamma}, \boldsymbol{\beta}, \mathbf{V}, \mathbf{U})$ é obtido maximizando-se a verossimilhança via BLUP (Henderson, 1975) em um primeiro passo e então estendido para se obter estimadores REML para $\left(\boldsymbol{\gamma}, \boldsymbol{\beta}, \mathbf{V}, \mathbf{U}, \theta_{1}, \theta_{2}\right)$. 


\section{Capítulo 2}

\section{Modelo de Chen-Ibrahim-Sinha com efeitos aleatórios}

Neste capítulo é apresentado o modelo de Chen-Ibrahim-Sinha com efeitos aleatórios, que visam avaliar se as respostas de indivíduos em uma mesma clínica estão correlacionadas, por exemplo, no tratamento de câncer de certa população que tem presença de imunes. É feita a caracterização do modelo e apresentada uma abordagem bayesiana e uma clássica para a obtenção de estimadores.

\subsection{Definindo o modelo}

Suponha que foram estudados casos em $M$ clínicas e que para a $k$-ésima clínica havia $n_{k}$ indivíduos, $k=1,2, \ldots, M$. Para $j=1,2, \ldots, n_{k}$, considere $N_{j k}$ o número de células susceptíveis a terem câncer que o indivíduo $j$ da clínica $k$ possui. Suponha ainda que $N_{j k}$ são independentes e com distribuição de Poisson com média $\theta_{j k}$. Para cada $k=1,2, \ldots, M$, defina o vetor $\mathbf{N}_{k}=\left[N_{1 k}, N_{2 k}, \ldots, N_{n_{k} k}\right]^{\top}$.

Seja $Z_{c j k}$ a variável aleatória para o tempo até que a $c$-ésima célula carcinogênica do indivíduo $j$ da clínica $k$ produza um câncer detectável. Suponha que as variáveis $Z_{c j k}$, são iid com função de distribuição $F(t \mid \boldsymbol{\lambda})=1-S(t \mid \boldsymbol{\lambda})$ e também são independentes de $N_{j k}$. Assim, o tempo para o aparecimento do câncer pode ser definido pela variável aleatória $T_{j k}=\min \left\{Z_{c j k}, 1 \leq c \leq N_{j k}\right\}$ mas $T_{j k}=\infty$ com probabilidade 1 , se $N_{j k}=0$.

Nesse trabalho, vamos utilizar formas paramétricas para $F(\cdot)$, como, por exemplo, Weibull. Neste caso, $\boldsymbol{\lambda}=(\lambda, \alpha)$ e $f(y \mid \boldsymbol{\lambda})=\alpha y^{\alpha-1} e^{\lambda-y^{\alpha} e^{\lambda}}$ e $S(y \mid \boldsymbol{\lambda})=e^{-y^{\alpha} e^{\lambda}}$, com $\alpha>0$ e $\lambda \in R$.

Como mostrado na seção 1.2, a fração de cura é obtida por $e^{-\theta_{j k}}$. Aqui, os valores de $\theta_{j k}$ dependerão das covariáveis $\mathbf{x}_{j k}$ por meio da relação $\theta_{j k}\left(\mathbf{w}_{j k}\right)=e^{\mathbf{w}_{j k}^{\top} \boldsymbol{\gamma}+V_{k}}$, em que $\mathbf{w}_{j k}=\left[\begin{array}{ll}1 & \mathbf{x}_{j k}^{\top}\end{array}\right]^{\top}$ são covariáveis fixas, $V_{k}$ é a variável correspondente ao efeito aleatório referente à clínica $k$, e vamos supor que $V_{1}, \ldots, V_{M}$ são independentes com $V_{k} \sim N\left(0, \sigma_{V}^{2}\right)$.

Para se determinar a verossimilhança, considere $C_{j k}$ o tempo de censura (à direita) para o $j$-ésimo indivíduo da clínica $k$. Seja $Y_{j k}=\min \left\{T_{j k}, C_{j k}\right\}$ e seja $\nu_{j k}$ o indicador de falha, ou seja, $\nu_{j k}=1$ se $y_{j k}$ é falha e 0 se for censura.

Assim, os dados observados são $D_{o b s}=(\mathbf{y}, \boldsymbol{\nu}, \mathbf{X})$ em que $\mathbf{y}=\left(y_{j k}\right)_{j=1, \ldots, n_{k}}^{k=1, \ldots, M}, \boldsymbol{\nu}=\left(\nu_{j k}\right)_{j=1, \ldots, n_{k}}^{k=1, \ldots, M}$ e $\mathbf{X}$ 
é a matriz de covariáveis.

Os dados completos são $D_{c}=(\mathbf{y}, \boldsymbol{\nu}, \mathbf{N}, \mathbf{V}, \mathbf{X})$ que incluem os dados observados e os dados não observados $(\mathbf{N}, \mathbf{V})$, com $\mathbf{N}=\left[\mathbf{N}_{1}, \mathbf{N}_{2}, \ldots, \mathbf{N}_{M}\right]$ e $\mathbf{V}=\left[V_{1}, V_{2}, \ldots, V_{M}\right]$. O vetor de parâmetros é dado por $\boldsymbol{\phi}=\left(\alpha, \boldsymbol{\beta}^{\top}, \boldsymbol{\gamma}^{\top}, \sigma_{V}^{2}\right)$.

Assim, utilizando (1.3), temos

$$
\begin{aligned}
f(\mathbf{y}, \mathbf{N}, \mathbf{V})= & f(\mathbf{y} \mid \mathbf{N}, \mathbf{V}) \cdot f(\mathbf{N} \mid \mathbf{V}) \cdot f(\mathbf{V}) \\
= & f(\mathbf{y} \mid \mathbf{N}, \mathbf{V}) \cdot f(\mathbf{N}) \cdot f(\mathbf{V}) \\
= & \prod_{k=1}^{M} \prod_{j=1}^{n_{k}}\left\{S\left(y_{j k} \mid \boldsymbol{\lambda}\right)^{N_{j k}-\nu_{j k}}\left[N_{j k} f\left(y_{j k} \mid \boldsymbol{\lambda}\right)\right]^{\nu_{j k}}\right\} \cdot \prod_{k=1}^{M} \prod_{j=1}^{n_{k}}\left\{\frac{\theta_{j k}^{N_{j k}}}{N_{j k} !} e^{-\theta_{j k}}\right\} . \\
& \cdot \prod_{k=1}^{M}\left\{\frac{1}{\sqrt{2 \pi \sigma_{V}^{2}}} e^{\frac{-V_{k}^{2}}{2 \sigma_{V}^{2}}}\right\} \\
= & \prod_{k=1}^{M}\left\{\frac{1}{\sqrt{2 \pi \sigma_{V}^{2}}} e^{\frac{-V_{k}^{2}}{2 \sigma_{V}^{2}}}\right\} . \\
& \cdot \prod_{k=1}^{M} \prod_{j=1}^{n_{k}}\left\{S\left(y_{j k} \mid \boldsymbol{\lambda}\right)^{N_{j k}-\nu_{j k}}\left[N_{j k} f\left(y_{j k} \mid \boldsymbol{\lambda}\right)\right]^{\nu_{j k}} \cdot \frac{\theta_{j k}^{N_{j k}}}{N_{j k} !} e^{-\theta_{j k}}\right\} \\
= & \prod_{k=1}^{M}\left\{* *_{k}\right\} \cdot \prod_{k=1}^{M} \prod_{j=1}^{n_{k}}\left\{\star_{j k}\right\}
\end{aligned}
$$

em que $*_{k}=\frac{1}{\sqrt{2 \pi \sigma_{V}^{2}}} e^{\frac{-V_{k}^{2}}{2 \sigma_{V}^{2}}}$ e $\star_{j k}=S\left(y_{j k} \mid \boldsymbol{\lambda}\right)^{N_{j k}-\nu_{j k}}\left[N_{j k} f\left(y_{j k} \mid \boldsymbol{\lambda}\right)\right]^{\nu_{j k}} \cdot \frac{\theta_{j k}^{N_{j k}}}{N_{j k} !} e^{-\theta_{j k}}$

Assim, a verossimilhança completa é dada por

$$
L\left(\boldsymbol{\phi} \mid D_{c}\right)=\prod_{k=1}^{M}\left\{\frac{e^{\frac{-V_{k}^{2}}{2 \sigma_{V}^{2}}}}{\sqrt{2 \pi \sigma_{V}^{2}}}\right\} \prod_{k=1}^{M} \prod_{j=1}^{n_{k}}\left\{S\left(y_{j k} \mid \boldsymbol{\lambda}\right)^{N_{j k}-\nu_{j k}}\left[N_{j k} f\left(y_{j k} \mid \boldsymbol{\lambda}\right)\right]^{\nu_{j k}} \frac{\theta_{j k}^{N_{j k}} e^{-\theta_{j k}}}{N_{j k} !}\right\}
$$

As variáveis correspondentes aos efeitos aleatórios $(\mathbf{V})$ e os números de células carcinogênicas $(\mathbf{N})$ são não observáveis. Então o estimador de máxima verossimilhança pode ser obtido maximizando-se a verossimilhança marginal, ou seja, integrando (somando) com relação às variáveis não observadas. Uma expressão simplificada pode ser obtida somando-se somente com relação ao vetor $\mathbf{N}$. 
Desse modo, tem-se que

$$
\begin{aligned}
\sum_{\mathbf{N}} L(\phi \mid \mathbf{y}, \boldsymbol{\nu}, \mathbf{V}, \mathbf{X}) & =\sum_{N}\left[\prod_{k=1}^{M}\left(*_{k}\right) \cdot \prod_{k=1}^{M} \prod_{j=1}^{n_{k}}\left(\star_{j k}\right)\right] \\
& =\prod_{k=1}^{M}\left(*_{k}\right) \cdot \prod_{k=1}^{M} \prod_{j=1}^{n_{k}} \sum_{N_{j k}=0}^{\infty}\left(\star_{j k}\right)
\end{aligned}
$$

Mas,

$$
\begin{aligned}
\sum_{N_{j k}=0}^{\infty}\left(\star_{j k}\right)= & \sum_{N_{j k}=0}^{\infty}\left\{S\left(y_{j k} \mid \boldsymbol{\lambda}\right)^{N_{j k}-\nu_{j k}}\left[N_{j k} f\left(y_{j k} \mid \boldsymbol{\lambda}\right)\right]^{\nu_{j k}} \cdot \frac{\theta_{j k}^{N_{j k}}}{N_{j k} !} e^{-\theta_{j k}}\right\} \\
= & \sum_{N_{j k}=0}^{\infty}\left\{S\left(y_{j k} \mid \boldsymbol{\lambda}\right)^{N_{j k}} S\left(y_{j k} \mid \boldsymbol{\lambda}\right)^{-\nu_{j k}}\left[N_{j k}\right]^{\nu_{j k}}\left[f\left(y_{j k} \mid \boldsymbol{\lambda}\right)\right]^{\nu_{j k}} \frac{\theta_{j k}^{N_{j k}}}{N_{j k} !} e^{-\theta_{j k}}\right. \\
& \left.\cdot e^{-\theta_{j k} S\left(y_{j k} \mid \boldsymbol{\lambda}\right)} e^{\theta_{j k} S\left(y_{j k} \mid \boldsymbol{\lambda}\right)}\right\} \\
= & \sum_{N_{j k}=0}^{\infty}\left\{\frac{\left[N_{j k}\right]^{\nu_{j k}}\left[\theta_{j k} S\left(y_{j k} \mid \boldsymbol{\lambda}\right)\right]^{N_{j k}} e^{-\theta_{j k} S\left(y_{j k} \mid \boldsymbol{\lambda}\right)}}{N_{j k} !}\right. \\
& \left.\cdot S\left(y_{j k} \mid \boldsymbol{\lambda}\right)^{-\nu_{j k}} e^{-\theta_{j k}} e^{\theta_{j k} S\left(y_{j k} \mid \boldsymbol{\lambda}\right)}\left[f\left(y_{j k} \mid \boldsymbol{\lambda}\right)\right]^{\nu_{j k}}\right\} \\
= & \theta_{j k} \nu_{j k} e^{-\theta_{j k}} e^{\theta_{j k} S\left(y_{j k} \mid \boldsymbol{\lambda}\right)}\left[f\left(y_{j k} \mid \boldsymbol{\lambda}\right)\right]^{\nu_{j k}}
\end{aligned}
$$

Portanto,

$$
\begin{aligned}
\sum_{\mathbf{N}} L(\boldsymbol{\phi} \mid \mathbf{y}, \boldsymbol{\nu}, \mathbf{V}, \mathbf{X}) & =\prod_{k=1}^{M}\left(*_{k}\right) \cdot \prod_{k=1}^{M} \prod_{j=1}^{n_{k}} \sum_{N_{j k}=0}^{\infty}\left(\star_{j k}\right) \\
& =\prod_{k=1}^{M}\left\{\frac{e^{\frac{-V_{k}^{2}}{2 \sigma_{V}^{2}}}}{\sqrt{2 \pi \sigma_{V}^{2}}}\right\} \prod_{k=1}^{M} \prod_{j=1}^{n_{k}}\left\{\theta_{j k}{ }^{\nu_{j k}} e^{-\theta_{j k}} e^{\theta_{j k} S\left(y_{j k} \mid \boldsymbol{\lambda}\right)}\left[f\left(y_{j k} \mid \boldsymbol{\lambda}\right)\right]^{\nu_{j k}}\right\}
\end{aligned}
$$

Se $y_{j k} \sim W$ eibull $\left(\alpha, \lambda_{j k}\right)$, então

$$
\begin{aligned}
& f\left(y_{j k} \mid \boldsymbol{\lambda}\right)=\alpha y_{j k}^{\alpha-1} e^{\lambda_{j k}-y_{j k}^{\alpha} e^{\lambda_{j k}}}, \\
& S\left(y_{j k} \mid \boldsymbol{\lambda}\right)=e^{-y_{j k}^{\alpha} e^{\lambda_{j k}}}
\end{aligned}
$$

em que

$$
\lambda_{j k}=\mathbf{x}_{j k}^{\top} \boldsymbol{\beta} .
$$


Assim,

$$
\begin{aligned}
\sum_{\mathbf{N}} L(\phi \mid \mathbf{y}, \boldsymbol{\nu}, \mathbf{V}, \mathbf{X})= & \prod_{k=1}^{M}\left\{\frac{e^{\frac{-V_{k}^{2}}{2 \sigma_{V}^{2}}}}{\sqrt{2 \pi \sigma_{V}^{2}}}\right\} . \\
& \cdot \prod_{k=1}^{M} \prod_{j=1}^{n_{k}}\left\{\left(e^{\mathbf{w}_{j k}^{\top} \boldsymbol{\gamma}+V_{k}}\right)^{\nu_{j k}} e^{-e^{\mathbf{w}_{j k}^{\top} \boldsymbol{\gamma}+V_{k}}} e^{\left(e^{\mathbf{w}_{j k}^{\top} \boldsymbol{\gamma}+V_{k}}\right)\left(e^{-y_{j k}^{\alpha} e^{\lambda_{j k}}}\right)}\left[\alpha y_{j k}^{\alpha-1} e^{\lambda_{j k}-y_{j k}^{\alpha} e^{\lambda_{j k}}}\right]^{\nu_{j k}}\right\} .
\end{aligned}
$$

\subsection{Inferência bayesiana}

Na abordagem bayesiana, a presença de quantidades não observáveis não representa um problema de inferência já que toda e qualquer quantidade desconhecida é uma variável aleatória. Assim, tanto para as quantidades de interesse (parâmetros), quanto para os efeitos aleatórios, é necessário obter sua distribuição a posteriori, ou seja, condicional aos dados observados. Basicamente, a distribuição a posteriori é proporcional ao produto da distribuição a priori para as quantidades desconhecidas e da função de verossimilhança. Uma completa apresentação da teoria bayesiana pode ser encontrada em Berger (1985).

Para o modelo de Chen-Ibrahim-Sinha com efeitos aleatórios, supondo prioris não informativas para $\boldsymbol{\gamma}$ e para $\boldsymbol{\beta}$, isto é, $\pi\left(\boldsymbol{\gamma}^{\top}\right) \propto 1$ e $\pi\left(\boldsymbol{\beta}^{\top}\right) \propto 1$, consideremos as distribuições a priori para o vetor de parâmetros $\phi$ :

$$
\pi(\phi)=\pi\left(\alpha, \boldsymbol{\beta}^{\top}, \boldsymbol{\gamma}^{\top}, \sigma_{V}^{2}\right) \propto \pi(\alpha) \pi\left(\sigma_{V}^{2}\right),
$$

em que as distribuições a priori são tais que

1. $\alpha \sim \operatorname{Gama}\left(\delta_{0}, \tau_{0}\right)$

$$
\pi(\alpha) \propto \alpha^{\delta_{0}-1} e^{-\tau_{0} \alpha}
$$

2. $\sigma_{V}^{2} \sim$ Gama Inversa $\left(a_{V}, b_{V}\right)$

$$
\pi\left(\sigma_{V}^{2}\right) \propto\left(\sigma_{V}^{2}\right)^{-\left(a_{V}+1\right)} e^{-\frac{b_{V}}{\sigma_{V}^{2}}} .
$$


Considerando a função de verossimilhança em (2.2), a distribuição a posteriori é dada por

$$
\begin{aligned}
\pi\left(\boldsymbol{\phi}, \mathbf{V} \mid D_{o b s}\right)= & \sum_{\mathbf{N}} L\left(\boldsymbol{\phi} \mid D_{c}\right) \pi(\boldsymbol{\phi}) \\
\propto & \prod_{k=1}^{M}\left\{\frac{e^{\frac{-V_{k}^{2}}{2 \sigma_{V}^{2}}}}{\sqrt{2 \pi \sigma_{V}^{2}}}\right\} \cdot \\
& \cdot \prod_{k=1}^{M} \prod_{j=1}^{n_{k}}\left\{\left(e^{\mathbf{w}_{j k}^{\top} \boldsymbol{\gamma}+V_{k}}\right)^{\nu_{j k}} e^{-e^{\mathbf{w}_{j k}^{\top} \gamma+V_{k}}} e^{\left.\left(e^{\mathbf{w}_{j k}^{\top} \gamma+V_{k}}\right)\left(e^{-y_{j k}^{\alpha} e^{\lambda_{j k}}}\right)\left[\alpha y_{j k}^{\alpha-1} e^{\lambda_{j k}-y_{j k}^{\alpha} e^{\lambda_{j k}}}\right]^{\nu_{j k}}\right\}}\right. \\
& \cdot \alpha^{\delta_{0}-1} e^{-\tau_{0} \alpha}\left(\sigma_{V}^{2}\right)^{-\left(a_{V}+1\right)} e^{-\frac{b_{V}}{\sigma_{V}^{2}}} .
\end{aligned}
$$

Uma forma de se obter estimativas dos parâmetros é via utilização de amostrador de Gibbs (Geman; Geman, 1984), método de simulação MCMC para aproximar as distribuições conjuntas e marginais, amostrando das distribuições condicionais. Assim, o próximo passo é tentar identificar as distribuições condicionais, a partir de (2.3).

Primeiro, vamos mostrar que $\sigma_{V}^{2} \mid \alpha, \boldsymbol{\gamma}, \boldsymbol{\beta}, \mathbf{V} \sim$ Gama Inversa $\left(a_{V}+\frac{1}{2}, b_{V}+\sum_{k=1}^{M} \frac{V_{k}^{2}}{2}\right)$. De fato:

$$
\begin{aligned}
\pi\left(\sigma_{V}^{2} \mid \alpha, \boldsymbol{\beta}, \boldsymbol{\gamma}, \mathbf{V}, D_{o b s}\right) & \propto \prod_{k=1}^{M}\left\{\frac{e^{\frac{-V_{k}^{2}}{2 \sigma_{V}^{2}}}}{\sqrt{2 \pi \sigma_{V}^{2}}}\right\} \cdot\left(\sigma_{V}^{2}\right)^{-\left(a_{V}+1\right)} e^{-\frac{b_{V}}{\sigma_{V}^{2}}} \\
& =\frac{1}{\sqrt{2 \pi \sigma_{V}^{2}}} \cdot\left(\sigma_{V}^{2}\right)^{-\left(a_{V}+1\right)} \cdot e^{-\frac{1}{\sigma_{V}^{2}} b_{V}} \cdot \prod_{k=1}^{M} e^{-\frac{1}{\sigma_{V}^{2}} \frac{V_{k}^{2}}{2}} \\
& \propto\left(\sigma_{V}^{2}\right)^{-1 / 2}\left(\sigma_{V}^{2}\right)^{-\left(a_{V}+1\right)} e^{-b_{V}\left(\sigma_{V}^{2}\right)^{-1}} e^{-\left(\sigma_{V}^{2}\right)^{-1} \sum_{k=1}^{M} \frac{V_{k}^{2}}{2}} \\
& =\left(\sigma_{V}^{2}\right)^{-\left(1 / 2+a_{V}+1\right)} e^{\left(\sigma_{V}^{2}\right)^{-1}\left[-b_{V}-\sum_{k=1}^{M} \frac{V_{k}^{2}}{2}\right]} \\
& =\left(\sigma_{V}^{2}\right)^{-\left(a_{V}+\frac{1}{2}\right)} e^{-\frac{1}{\sigma_{V}^{2}}\left[b_{V}+\sum_{k=1}^{M} \frac{V_{k}^{2}}{2}\right]}
\end{aligned}
$$

o que caracteriza a distribuição de $\sigma_{V}^{2} \mid \alpha, \boldsymbol{\beta}^{\top}, \boldsymbol{\gamma}^{\top}, \mathbf{V}$ como sendo uma Gama Inversa com parâmetros Gama Inversa $\left(a_{V}+\frac{1}{2}, b_{V}+\sum_{k=1}^{M} \frac{V_{k}^{2}}{2}\right)$.

Mas, nem todas as distribuições condicionais terão forma fechada. Nestes casos, uma alternativa é usar algum algoritmo de aceitação/rejeição ou "Metropolis-Hastings"(Chib \& Greenberg, 1995).

Seja $d_{j k}=\left(e^{\mathbf{w}_{j k}^{\top} \boldsymbol{\gamma}+V_{k}}\right)^{\nu_{j k}} e^{-e^{\mathbf{w}_{j k}^{\top} \boldsymbol{\gamma}+V_{k}}}$. A distribuição a posteriori condicional de $\alpha \mid \boldsymbol{\beta}^{\top}, \boldsymbol{\gamma}^{\top}, \sigma_{V}^{2}, \mathbf{V}$, é dada por 


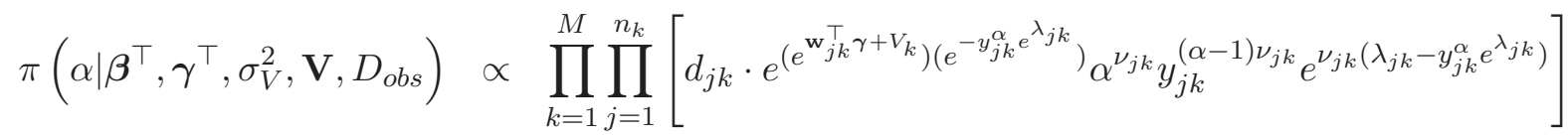

$$
\begin{aligned}
& \cdot \alpha^{\delta_{0}-1} e^{-\tau_{0} \alpha} \\
& =\prod_{k=1}^{M} \prod_{j=1}^{n_{k}}\left[d_{j k} \alpha^{\nu_{j k}} y_{j k}^{\alpha \nu_{j k}} y_{j k}^{-\nu_{j k}} e^{e^{\mathbf{w}_{j k}^{\top} \gamma+V_{k}-y_{j k}^{\alpha} e^{\lambda_{j k}}}+\nu_{j k} \lambda_{j k}-\nu_{j k} y_{j k}^{\alpha} e^{\lambda_{j k}}}\right] \\
& \cdot \alpha^{\delta_{0}-1} e^{-\tau_{0} \alpha} \\
& \propto \quad \alpha^{\delta_{0}-1} \cdot e^{-\tau_{0} \alpha} \cdot \alpha^{\sum_{k=1}^{M} \sum_{j=1}^{n_{k}} \nu_{j k}} \cdot y_{j k}^{\alpha \sum_{k=1}^{M} \sum_{j=1}^{n_{k}} \nu_{j k}} . \\
& \cdot \exp \left\{\sum_{k=1}^{M} \sum_{j=1}^{n_{k}} e^{\mathbf{w}_{j k}^{\top} \boldsymbol{\gamma}+V_{k}-y_{j k}^{\alpha} e^{\lambda_{j k}}}+\nu_{j k} \lambda_{j k}-\nu_{j k} y_{j k}^{\alpha} e^{\lambda_{j k}}\right\} \\
& =\alpha^{\delta_{0}-1+\sum_{k=1}^{M} \sum_{j=1}^{n_{k}} \nu_{j k}} \cdot \exp \left\{\sum_{k=1}^{M} \sum_{j=1}^{n_{k}} \nu_{j k} \lambda_{j k}\right\} \text {. } \\
& \cdot \exp \left\{-\tau_{0} \alpha+\alpha \sum_{k=1}^{M} \sum_{j=1}^{n_{k}} \nu_{j k} \cdot \ln y_{j k}\right. \\
& \left.+\sum_{k=1}^{M} \sum_{j=1}^{n_{k}}\left[e^{\mathbf{w}_{j k}^{\top} \boldsymbol{\gamma}+V_{k}-y_{j k}^{\alpha} e^{\lambda_{j k}}}-\nu_{j k} y_{j k}^{\alpha} e^{\lambda_{j k}}\right]\right\} \\
& \propto \quad \alpha^{\left(\delta_{0}+\sum_{k=1}^{M} \sum_{j=1}^{n_{k}} \nu_{j k}\right)-1} \cdot \exp \left\{-\alpha\left(\tau_{0}-\sum_{k=1}^{M} \sum_{j=1}^{n_{k}} \nu_{j k} \ln y_{j k}\right)\right\} \text {. } \\
& \cdot \exp \left\{\sum_{k=1}^{M} \sum_{j=1}^{n_{k}}\left[e^{\mathbf{w}_{j k}^{\top} \boldsymbol{\gamma}+V_{k}-y_{j k}^{\alpha} e^{\lambda_{j k}}}-\nu_{j k} y_{j k}^{\alpha} e^{\lambda_{j k}}\right]\right\} \\
& =\operatorname{Gama}\left(\delta_{0}+\sum_{k=1}^{M} \sum_{j=1}^{n_{k}} \nu_{j k} ; \quad \tau_{0}-\sum_{k=1}^{M} \sum_{j=1}^{n_{k}} \nu_{j k} \ln y_{j k}\right) \text {. } \\
& \cdot \exp \left\{\sum_{k=1}^{M} \sum_{j=1}^{n_{k}}\left[e^{\mathbf{w}_{j k}^{\top} \boldsymbol{\gamma}+V_{k}-y_{j k}^{\alpha} e^{\lambda_{j k}}}-\nu_{j k} y_{j k}^{\alpha} e^{\lambda_{j k}}\right]\right\} \text {, }
\end{aligned}
$$

desde que $\tau_{0}-\sum_{k=1}^{M} \sum_{j=1}^{n_{k}}\left(\nu_{j k} \ln y_{j k}\right)>0$

Para a distribuição a posteriori condicional de $\mathbf{V} \mid \alpha, \boldsymbol{\beta}, \boldsymbol{\gamma}, \sigma_{V}^{2}$ tem-se: 


$$
\begin{aligned}
\pi\left(\mathbf{V} \mid \alpha, \boldsymbol{\beta}, \boldsymbol{\gamma}, \sigma_{V}^{2}, D_{o b s}\right) & \propto \prod_{k=1}^{M} \frac{e^{\frac{-V_{k}^{2}}{2 \sigma_{V}^{2}}}}{\sqrt{2 \pi \sigma_{V}^{2}}} \cdot \prod_{k=1}^{M} \prod_{j=1}^{n_{k}}\left[e^{V_{k} \nu_{j k}} \cdot e^{-e^{\mathbf{w}_{j k}^{\top} \gamma+V_{k}}} \cdot e^{e^{\mathbf{w}_{j k}^{\top} \gamma+V_{k}-y_{j k}^{\alpha} e^{\lambda_{j k}}}}\right] \\
& \propto \prod_{k=1}^{M}\left\{\frac{e^{\frac{-V_{k}^{2}}{2 \sigma_{V}^{2}}}}{\sqrt{2 \pi \sigma_{V}^{2}}} \cdot \prod_{j=1}^{n_{k}}\left[e^{V_{k} \nu_{j k}} \cdot e^{-e^{\mathbf{w}_{j k}^{\top} \gamma+V_{k}}} \cdot e^{e^{\mathbf{w}_{j k}^{\top} \gamma+V_{k}-y_{j k}^{\alpha} e^{\lambda} j k}}\right]\right\} \\
& =\prod_{k=1}^{M} \pi\left(V_{k} \mid \alpha, \boldsymbol{\beta}, \boldsymbol{\gamma}, \sigma_{V}^{2}\right)
\end{aligned}
$$

Note que a última igualdade é verdadeira visto que $\mathbf{V}=\left[V_{1}, V_{2}, \ldots, V_{M}\right]$ e para $k=1,2, \ldots, M$ tem-se $V_{k}$ iid. Assim, para cada $k=1,2, \ldots, M$

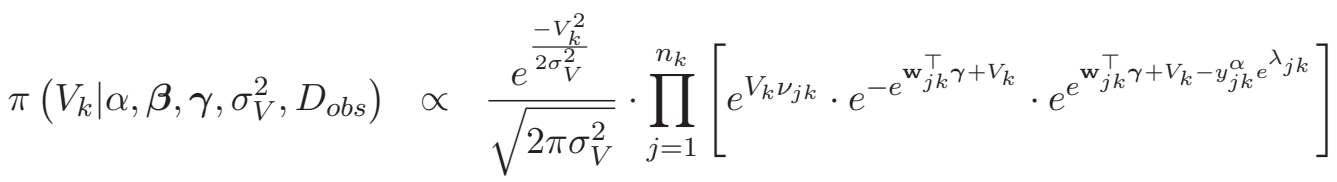

$$
\begin{aligned}
& =\exp \left\{\frac{-V_{k}^{2}}{2 \sigma_{V}^{2}}+\sum_{j=1}^{n_{k}}\left(V_{k} \nu_{j k}-e^{\mathbf{w}_{j k}^{\top} \boldsymbol{\gamma}+V_{k}}+e^{\mathbf{w}_{j k}^{\top} \boldsymbol{\gamma}+V_{k}} \cdot e^{-y_{j k}^{\alpha} e^{\lambda_{j k}}}\right)\right\} \\
& =\exp \left\{\frac{-V_{k}^{2}}{2 \sigma_{V}^{2}}\right\} \cdot \exp \left\{\sum_{j=1}^{n_{k}}\left[V_{k} \nu_{j k}-e^{\mathbf{w}_{j k}^{\top} \boldsymbol{\gamma}+V_{k}} \cdot\left(1-e^{-y_{j k}^{\alpha} e^{\lambda_{j k}}}\right)\right]\right\} \\
& =\operatorname{Normal}\left(0, \sigma_{V}^{2}\right) \cdot \exp \left\{\sum_{j=1}^{n_{k}}\left[V_{k} \nu_{j k}-e^{\mathbf{w}_{j k}^{\top} \boldsymbol{\gamma}+V_{k}} \cdot\left(1-e^{-y_{j k}^{\alpha} e^{\lambda_{j k}}}\right)\right]\right\}
\end{aligned}
$$

Para a distribuição a posteriori condicional de $\boldsymbol{\gamma}^{\top} \mid \alpha, \boldsymbol{\beta}^{\top}, \sigma_{V}^{2}$, $\mathbf{V}$ tem-se:

$$
\begin{aligned}
\pi\left(\boldsymbol{\gamma}^{\top} \mid \alpha, \boldsymbol{\beta}^{\top}, \sigma_{V}^{2}, \mathbf{V}, D_{\text {obs }}\right) & \propto \prod_{k=1}^{M} \prod_{j=1}^{n_{k}}\left[e^{\nu_{j k}\left(\mathbf{w}_{j k}^{\top} \boldsymbol{\gamma}+V_{k}\right)} e^{-e^{\mathbf{w}_{j k}^{\top} \boldsymbol{\gamma}+V_{k}}} e^{\left(e^{\left.\mathbf{w}_{j k}^{\top} \boldsymbol{\gamma}+V_{k}-y_{j k}^{\alpha} e^{\lambda_{j k}}\right)}\right]=}\right. \\
& =\exp \left\{\sum_{k=1}^{M} \sum_{j=1}^{n_{k}} \nu_{j k}\left(\mathbf{w}_{j k}^{\top} \boldsymbol{\gamma}+V_{k}\right)-e^{\mathbf{w}_{j k}^{\top} \boldsymbol{\gamma}+V_{k}}+e^{\mathbf{w}_{j k}^{\top} \boldsymbol{\gamma}+V_{k}-y_{j k}^{\alpha} e^{\lambda_{j k}}}\right\}= \\
& =\exp \left\{\sum_{k=1}^{M} \sum_{j=1}^{n_{k}}\left(\left(w_{j k}^{\top} \boldsymbol{\gamma}+V_{k}\right) \nu_{j k}-e^{\left(w_{j k}^{\top} \boldsymbol{\gamma}+V_{k}\right)}\left(-1-e^{\left.-y_{j k}^{\alpha} e^{\lambda_{j k}}\right)}\right)\right\}\right.
\end{aligned}
$$


Lembrando que $\lambda_{j k}=\mathbf{x}_{j k}^{\top} \boldsymbol{\beta}$, para a distribuição a posteriori condicional de $\boldsymbol{\beta}^{\top} \mid \alpha, \boldsymbol{\gamma}^{\top}, \sigma_{V}^{2}, \mathbf{V}$ tem-se:

$$
\begin{aligned}
\pi\left(\boldsymbol{\beta}^{\top} \mid \alpha, \boldsymbol{\gamma}^{\top}, \sigma_{V}^{2}, \mathbf{V}, D_{o b s}\right) & \propto \prod_{k=1}^{M} \prod_{j=1}^{n_{k}} \exp \left\{e^{\mathbf{w}_{j k}^{\top} \boldsymbol{\gamma}+V_{k}-y_{j k}^{\alpha} e^{\lambda_{j k}}}\right\} e^{\nu_{j k}\left(\lambda_{j k}-y_{j k}^{\alpha} e^{\lambda_{j k}}\right)}= \\
& =\prod_{k=1}^{M} \prod_{j=1}^{n_{k}} \exp \left\{e^{\mathbf{w}_{j k}^{\top} \boldsymbol{\gamma}+V_{k}-y_{j k}^{\alpha} e^{\lambda_{j k}}}+\nu_{j k} \lambda_{j k}-\nu_{j k} y_{j k}^{\alpha} e^{\lambda_{j k}}\right\} \\
& =\exp \left\{\sum_{k=1}^{M} \sum_{j=1}^{n_{k}}\left(e^{\mathbf{w}_{j k}^{\top} \boldsymbol{\gamma}+V_{k}-y_{j k}^{\alpha} e^{\mathbf{x}_{j k}^{\top} \boldsymbol{\beta}}}+\nu_{j k} \mathbf{x}_{j k}^{\top} \boldsymbol{\beta}-\nu_{j k} y_{j k}^{\alpha} e^{\mathbf{x}_{j k}^{\top} \boldsymbol{\beta}}\right)\right\}
\end{aligned}
$$

Para se avaliar se a convergência do modelo está adequada, Gelman e Rubin (1992) e Cowless e Carlin (1996) sugerem, dentre outras, a utilização do valor $\sqrt{\hat{R}}$. Para estes cálculos somente são considerados os últimos $50 \%$ valores da iteração, de modo a se obter uma melhor convergência.

Considere $m$ o número de cadeias utilizadas com valores iniciais distintos, em que cada uma apresenta $2 n$ valores, mas para o diagnóstico de convergência, serão utilizados os $n$ últimos valores. Sejam $B / n$ a variância entre as médias $\left(\bar{u}_{i}\right)_{i=1,2, \ldots, m}$ das $m$ cadeias, cada uma baseada em $n$ valores de $u$ e $W$ a média das variâncias $\left(s_{i}^{2}\right)_{i=1,2, \ldots, m}$ entre as cadeias, cada uma baseada em $n-1$ graus de liberdade. Ou seja,

$$
\begin{aligned}
\frac{B}{n} & =\sum_{i=1}^{m} \frac{\left(\bar{u}_{i}-\bar{u}_{. .}\right)^{2}}{m-1} \\
W= & =\sum_{i=1}^{m} \frac{s_{i}^{2}}{m}
\end{aligned}
$$

Considere ainda

$$
\begin{aligned}
\hat{\sigma^{2}=} & \frac{(n-1) W}{n}+\frac{B}{n} \\
\hat{v}= & \hat{\sigma}+\frac{B}{m n} \\
\hat{\operatorname{Var}}(\hat{v})= & \left(\frac{n-1}{n}\right)^{2} \frac{\hat{s}_{i}^{2}}{m}+\left(\frac{m+1}{m n}\right)^{2} \frac{2 B^{2}}{m-1}+ \\
& +\frac{2(m+1)(n-1)}{m^{2} n}\left(\hat{\operatorname{Cov}}\left(s_{i}^{2}, \bar{u}_{i}^{2}\right)-2 \bar{u}_{. .} \hat{\operatorname{Cov}}\left(s_{i}^{2}, \bar{u}_{i}\right)\right) \\
d f= & \frac{2 \hat{v}^{2}}{\hat{\operatorname{Var}}(\hat{v})}
\end{aligned}
$$


Assim, define-se

$$
\sqrt{\hat{R}}=\sqrt{\frac{n-1}{n}+\frac{m+1}{m} \frac{B / n}{W} \frac{d f}{d f-2}} .
$$

Valores de $\sqrt{\hat{R}}$ próximos a 1 para todas as estimativas de interesse indicam que houve convergência da cadeia e que foi atingida a distribuição de cada estimador. Para maiores detalhes, ver Gelman e Rubin (1992).

\subsection{Análise clássica}

A equação (2.2) não é exatamente uma verossimilhança devido à presença de quantidades não observadas, dadas pelos efeitos aleatórios V. Um modo de se encontrar estimadores para os parâmetros seria a utilização do algoritmo EM (McLachan e Krishnan, 1997). Mas, as integrais envolvidas não são tratáveis de um modo analítico direto, sendo necessário o uso de alguma técnica, por exemplo numérica, de aproximação de integrais.

Outro método possível de estimação se baseia em primeiro calcular o melhor preditor linear nãoviesado (best linear unbiased predictor - BLUP) de Henderson (1975) e estendê-lo para se obter estimadores de máxima verossimilhança residual (residual maximum likelihood estimators - REML) aproximados (Thompson, 1980). Informações complementares podem ser encontradas em McGilchrist (1994) e em McGilchrist e Yau (1995).

Para se encontrar o BLUP, considere:

- $\boldsymbol{\phi}_{E}=\left(\alpha, \boldsymbol{\beta}^{\top}, \boldsymbol{\gamma}^{\top}, \sigma_{V}^{2}, \mathbf{V}^{\top}\right)^{\top}$

- $l$ o logaritmo neperiano de (2.2), ou seja, $l=\ln \left(\sum_{\mathbf{N}} L(\phi \mid \mathbf{y}, \boldsymbol{\nu}, \mathbf{V}, \mathbf{X})\right)$;

- U o vetor escore de $l$ em $\phi_{E}$, ou seja, o vetor das primeiras derivadas de $l$ em relação à $\phi_{E}$;

- $H$ a matriz hessiana de $l$ em $\phi_{E}$, ou seja, a matriz das segundas derivadas de $l$ em relação à $\phi_{E}$.

O BLUP para $\mathbf{V}$ e estimadores para $\phi$ serão encontrados a partir das raízes do vetor escore $\mathbf{U}$. 
Para $l$ tem-se:

$$
\begin{aligned}
l= & \ln \left(\sum_{\mathbf{N}} L(\phi \mid \mathbf{y}, \boldsymbol{\nu}, \mathbf{V}, \mathbf{X})\right) \\
= & \sum_{k=1}^{M}\left(\frac{-1}{2} \ln \left(2 \pi \sigma_{V}^{2}\right)-\frac{V_{K}^{2}}{2 \pi \sigma_{V}^{2}}\right)+ \\
& +\sum_{k=1}^{M} \sum_{j=1}^{n_{k}}\left\{\left(\mathbf{w}_{j k}^{\top} \gamma+V_{k}\right) \cdot \nu_{j k}-\exp \left(\mathbf{w}_{j k}^{\top} \gamma+V_{k}\right)+\exp \left(\mathbf{w}_{j k}^{\top} \gamma+V_{k}\right) \cdot \exp \left(-y_{j k}^{\alpha} e^{\lambda_{j k}}\right)+\right. \\
& \left.+\nu_{j k} \cdot\left(\ln (\alpha)+(\alpha-1) \ln \left(y_{j k}\right)+\lambda_{j k}-y_{j k}^{\alpha} e^{\lambda_{j k}}\right)\right\}
\end{aligned}
$$

A derivada de $l$ em $\alpha$ é dada por

$$
\begin{aligned}
\frac{\partial l}{\partial \alpha}= & \sum_{k=1}^{M} \sum_{j=1}^{n_{k}}\left\{\exp \left(\mathbf{w}_{j k}^{\top} \boldsymbol{\gamma}+V_{k}\right) \cdot \exp \left(-y_{j k}^{\alpha} e^{\lambda_{j k}}\right) \cdot \exp \left(\lambda_{j k}\right) \cdot\left(-\alpha \cdot y_{j k}^{\alpha-1}\right)+\right. \\
& \left.+\nu_{j k} \cdot\left[\frac{1}{\alpha}+\ln \left(y_{j k}\right)-e^{\lambda_{j k}} \cdot\left(-\alpha \cdot y_{j k}^{\alpha-1}\right)\right]\right\}
\end{aligned}
$$

A derivada de $l$ em $\boldsymbol{\beta}$ é dada por

$$
\frac{\partial l}{\partial \boldsymbol{\beta}}=\sum_{k=1}^{M} \sum_{j=1}^{n_{k}}\left\{\exp \left(\mathbf{w}_{j k}^{\top} \boldsymbol{\gamma}+V_{k}\right) \cdot \exp \left(-y_{j k}^{\alpha} e^{\lambda_{j k}}\right) \cdot\left(-y_{j k}^{\alpha} e^{\lambda_{j k}} x_{j k}\right)+\nu_{j k} x_{j k}-\nu_{j k} y_{j k}^{\alpha} e^{\lambda_{j k}} x_{j k}\right\}
$$

A derivada de $l$ em $\gamma$ é dada por

$$
\begin{aligned}
\frac{\partial l}{\partial \boldsymbol{\gamma}} & =\sum_{k=1}^{M} \sum_{j=1}^{n_{k}}\left\{\mathbf{w}_{j k} \cdot \nu_{j k}-\exp \left(\mathbf{w}_{j k}^{\top} \boldsymbol{\gamma}+V_{k}\right) \cdot \mathbf{w}_{j k}+\exp \left(\mathbf{w}_{j k}^{\top} \boldsymbol{\gamma}+V_{k}\right) \cdot \mathbf{w}_{j k} \cdot \exp \left(-y_{j k}^{\alpha} e^{\lambda_{j k}}\right)\right\} \\
& =\sum_{k=1}^{M} \sum_{j=1}^{n_{k}}\left\{\mathbf{w}_{j k} \cdot \nu_{j k}-\exp \left(\mathbf{w}_{j k}^{\top} \boldsymbol{\gamma}+V_{k}\right) \cdot \mathbf{w}_{j k} \cdot\left(1-\exp \left(-y_{j k}^{\alpha} e^{\lambda_{j k}}\right)\right)\right\} \\
& =\sum_{k=1}^{M} \sum_{j=1}^{n_{k}} \mathbf{w}_{j k} \cdot \nu_{j k}-\sum_{k=1}^{M} \sum_{j=1}^{n_{k}}\left\{\exp \left(\mathbf{w}_{j k}^{\top} \boldsymbol{\gamma}+V_{k}\right) \cdot \mathbf{w}_{j k} \cdot\left(1-\exp \left(-y_{j k}^{\alpha} e^{\lambda_{j k}}\right)\right)\right\}
\end{aligned}
$$

A derivada de $l$ em $\sigma_{V}^{2}$ é dada por

$$
\begin{aligned}
\frac{\partial l}{\partial \sigma_{V}^{2}} & =\sum_{k=1}^{M}\left\{\frac{-1}{2} \frac{1}{\sigma_{V}^{2}}-\frac{V_{k}^{2}}{2}\left(\frac{-1}{\left(\sigma_{V}^{2}\right)^{2}}\right)\right\}=\sum_{k=1}^{M}\left\{\frac{-1}{2 \sigma_{V}^{2}}+\frac{V_{k}^{2}}{2\left(\sigma_{V}^{2}\right)^{2}}\right\}=\frac{1}{2 \sigma_{V}^{2}} \sum_{k=1}^{M}\left\{-1+\frac{V_{k}^{2}}{\sigma_{V}^{2}}\right\} \\
& =\frac{1}{2 \sigma_{V}^{2}}\left\{\sum_{k=1}^{M}(-1)+\frac{1}{\sigma_{V}^{2}} \sum_{k=1}^{M} V_{k}^{2}\right\}=\frac{1}{2 \sigma_{V}^{2}}\left\{-M+\frac{1}{\sigma_{V}^{2}} \sum_{k=1}^{M} V_{k}^{2}\right\}=\frac{-M \sigma_{V}^{2}+\sum_{k=1}^{M} V_{k}^{2}}{2 \sigma_{V}^{2}}
\end{aligned}
$$


Finalmente, a derivada de $l$ em $\mathbf{V}$ é o vetor das derivadas de $l$ em $V_{k}$, em que cada componente é dada por

$$
\begin{aligned}
\frac{\partial l}{\partial V_{k}} & =\frac{-1}{2 \sigma_{V}^{2}} \cdot 2 V_{k}+\sum_{j=1}^{n_{k}}\left\{\nu_{j k}-\exp \left(\mathbf{w}_{j k}^{\top} \boldsymbol{\gamma}+V_{k}\right) \cdot 1+\exp \left(\mathbf{w}_{j k}^{\top} \boldsymbol{\gamma}+V_{k}\right) \cdot \exp \left(-y_{j k}^{\alpha} e^{\lambda_{j k}}\right)\right\} \\
& =\frac{-V_{k}}{\sigma_{V}^{2}}+\sum_{j=1}^{n_{k}} \nu_{j k}-\sum_{j=1}^{n_{k}}\left\{\exp \left(\mathbf{w}_{j k}^{\top} \boldsymbol{\gamma}+V_{k}\right) \cdot\left(1-\exp \left(-y_{j k}^{\alpha} e^{\lambda_{j k}}\right)\right)\right\} \\
& =\frac{-V_{k}}{\sigma_{V}^{2}}+\sum_{j=1}^{n_{k}} \nu_{j k}-\sum_{j=1}^{n_{k}}\left\{e^{V_{k}} \cdot e^{\mathbf{w}_{j k}^{\top} \boldsymbol{\gamma}} \cdot\left(1-\exp \left(-y_{j k}^{\alpha} e^{\lambda_{j k}}\right)\right)\right\} \\
& =\frac{-V_{k}}{\sigma_{V}^{2}}+\sum_{j=1}^{n_{k}} \nu_{j k}-e^{V_{k}} \cdot \sum_{j=1}^{n_{k}}\left\{e^{\mathbf{w}_{j k}^{\top} \boldsymbol{\gamma}} \cdot\left(1-\exp \left(-y_{j k}^{\alpha} e^{\lambda_{j k}}\right)\right)\right\}
\end{aligned}
$$

O vetor escore $\mathbf{U}$ é dado por $\mathbf{U}=\left(\frac{\partial l}{\partial \alpha}, \frac{\partial l}{\partial \boldsymbol{\beta}^{\top}}, \frac{\partial l}{\partial \boldsymbol{\gamma}^{\top}}, \frac{\partial l}{\partial \sigma_{V}^{2}}, \frac{\partial l}{\partial \mathbf{V}^{\top}}\right)^{\top}$.

Para se encontrar o BLUP, deve-se encontrar a solução de $\mathbf{U}=\overrightarrow{0}$. Mas, isto não é de fácil solução. Entretanto, note que

$$
\begin{aligned}
\frac{\partial l}{\partial \sigma_{V}^{2}} & =0 \Longleftrightarrow \frac{-M \sigma_{V}^{2}+\sum_{k=1}^{M} V_{k}^{2}}{2 \sigma_{V}^{2}}=0 \Longleftrightarrow-M \sigma_{V}^{2}+\sum_{k=1}^{M} V_{k}^{2}=0 \Longleftrightarrow \\
\sigma_{V}^{2} & =\frac{\sum_{k=1}^{M} V_{k}^{2}}{M}
\end{aligned}
$$

Como a solução das demais equações não são analitamente tratáveis, uma alternativa é utilizar o método de Newton-Raphson, que diz que, se $\phi_{E}(t+1)$ são as estimativas em $\phi_{E}$ no passo $t+1$, se $\phi_{E}(t)$ são as estimativas em $\phi_{E}$ no passo $t$, se $\mathbf{U}(t)$ é o vetor escore calculado em $\phi_{E}(t)$ e se $H(t)$ é a matriz hessiana calculada em $\phi_{E}(t)$, então

$$
\phi_{E}(t+1)=\phi_{E}(t)-H(t)^{-1} \cdot \mathbf{U}(t)
$$

em que (2.5) é inicializado com valores para $\phi_{E}$ e deve ser calculado até que $\left\|\phi_{E}(t+1)-\phi_{E}(t)\right\|<\varepsilon$, $\operatorname{com} \varepsilon$ suficientemente pequeno. Pode-se, por exemplo, usar $\varepsilon=10^{-3}$.

Se $\tilde{\mathbf{V}}$ são os preditores para $\mathbf{V}$, e $\tilde{\phi_{E}}$ são as estimativas para $\phi_{E}$, obtidos por BLUP e se a matriz hessiana-inversa calculada em $\tilde{\phi_{E}}$ é decomposta relativamente a $\left(\alpha, \boldsymbol{\beta}^{\top}, \boldsymbol{\gamma}^{\top}\right)\left|\sigma_{V}^{2}\right| \mathbf{V}^{\top}$ por

$$
H^{-1}=\left[\begin{array}{ccc}
A_{11} & A_{12} & A_{13} \\
A_{21} & A_{22} & A_{23} \\
A_{31} & A_{32} & A_{33}
\end{array}\right]
$$


então, segundo McGilchrist e Yau (1995) e Yau e Ng (2001), as estimativas para $\sigma_{V}^{2}$ via REML serão calculadas por

$$
\hat{\sigma}_{V}^{2}=\frac{1}{M}\left(-\operatorname{tr}\left(A_{33}\right)+\tilde{\mathbf{V}}^{\top} \tilde{\mathbf{V}}\right)
$$

A variância de $\hat{\sigma_{V}^{2}}$ é determinada por

$$
\operatorname{Var}\left(\hat{\sigma_{V}^{2}}\right)=2\left(\frac{1}{\sigma_{V}^{2}}\left(M+2 \frac{1}{\sigma_{V}^{2}} \operatorname{tr}\left(A_{33}\right)\right)-\frac{1}{\sigma_{V}^{4}} \operatorname{tr}\left(A_{33}^{2}\right) .\right)^{-1}
$$

As variâncias dos demais parâmetros são obtidas através da matriz informação de Fisher, ou seja, são dadas por

$$
\operatorname{Var}\left(\left[\alpha, \boldsymbol{\beta}^{\top}, \boldsymbol{\gamma}^{\top}\right]^{\top}\right)=-\left[A_{11}\right]
$$




\section{Capítulo 3}

\section{Estudos de simulação - modelo de Chen-Ibrahim-Sinha com efeitos aleatórios}

Neste capítulo são apresentados estudos de simulação feitos para o Modelo de Chen-IbrahimSinha com efeitos aleatórios, apresentado no Capítulo 2, tanto para a abordagem bayesiana quanto para a clássica.

Para este estudo foram utilizados os programas R (versão 2.5.1) (Venables et al., 2008), Winbugs (versão 1.4) (Spiegelhalter, 2007) e Maple (versão 10) (Maplesoft, 2005).

\subsection{Características}

Para este estudo, em uma primeira situação, são assumidas as mesmas condições básicas apresentadas em Yau-Ng (2001):

- $M=10$, ou seja, 10 clínicas;

- $n_{k}=15$ para todo $k=1,2, \ldots, M$, ou seja, cada clínica possui 15 indivíduos;

- em cada clínica, 5 pacientes são aleatoriamente designados para o grupo de tratamento $\left(x_{j k}=1\right)$ e os 10 restantes, para o grupo controle $\left(x_{j k}=0\right)$;

- 12 grupos de parâmetros a serem analisados:

1. $\left(\alpha=1 ; \sigma_{V}^{2}=0,5\right.$; Censura $\left.=500 ; \beta_{1}=-0,5 ; \gamma_{0}=0 ; \gamma_{1}=0,5\right)$,

2. $\left(\alpha=1,3 ; \sigma_{V}^{2}=0,5\right.$; Censura $\left.=500 ; \beta_{1}=-0,5 ; \gamma_{0}=0 ; \gamma_{1}=0,5\right)$,

3. $\left(\alpha=1 ; \sigma_{V}^{2}=1 ;\right.$ Censura $\left.=500 ; \beta_{1}=-0,5 ; \gamma_{0}=0 ; \gamma_{1}=0,5\right)$,

4. $\left(\alpha=1 ; \sigma_{V}^{2}=0,5\right.$; Censura $\left.=300 ; \beta_{1}=-0,5 ; \gamma_{0}=0 ; \gamma_{1}=0,5\right)$,

5. $\left(\alpha=1 ; \sigma_{V}^{2}=0,5\right.$; Censura $\left.=500 ; \beta_{1}=-0,5 ; \gamma_{0}=-0,5 ; \gamma_{1}=0,5\right)$,

6. $\left(\alpha=1,3 ; \sigma_{V}^{2}=0,5\right.$; Censura $\left.=500 ; \beta_{1}=-0,5 ; \gamma_{0}=-0,5 ; \gamma_{1}=0,5\right)$,

7. $\left(\alpha=1 ; \sigma_{V}^{2}=1 ;\right.$ Censura $\left.=500 ; \beta_{1}=-0,5 ; \gamma_{0}=-0,5 ; \gamma_{1}=0,5\right)$, 

8. $\left(\alpha=1 ; \sigma_{V}^{2}=0,5 ;\right.$ Censura $\left.=300 ; \beta_{1}=-0,5 ; \gamma_{0}=-0,5 ; \gamma_{1}=0,5\right)$,
9. $\left(\alpha=1 ; \sigma_{V}^{2}=0,5 ;\right.$ Censura $\left.=500 ; \beta_{1}=-1 ; \gamma_{0}=-1 ; \gamma_{1}=1\right)$,
10. $\left(\alpha=1,3 ; \sigma_{V}^{2}=0,5\right.$; Censura $\left.=500 ; \beta_{1}=-1 ; \gamma_{0}=-1 ; \gamma_{1}=1\right)$,
11. $\left(\alpha=1 ; \sigma_{V}^{2}=1 ;\right.$ Censura $\left.=500 ; \beta_{1}=-1 ; \gamma_{0}=-1 ; \gamma_{1}=1\right)$,
12. $\left(\alpha=1 ; \sigma_{V}^{2}=0,5\right.$; Censura $\left.=300 ; \beta_{1}=-1 ; \gamma_{0}=-1 ; \gamma_{1}=1\right)$;

- 50 simulações para cada grupo de parâmetros.

Em um segundo momento foi estudado o caso em $n_{k}=50$ para todo $k=1,2, \ldots, 15$ com as demais condições mantidas. Ou seja, a alteração feita nos permite analisar melhor resultados assintóticos, já que houve um aumento no número de indivíduos de cada clínica.

O que se espera é que:

- o desempenho das estimativas melhore, quando $\alpha$ aumente de 1 para 1,3 , porque quanto menor o valor de $\alpha$, maior será a média da distribuição de $Z_{c j k}$, Weibull $(\alpha, \lambda)$.

- uma diminuição na censura (de 500 para 300) piore a desempenho das estimativas, já que esta diminuição poderá fazer com que se tenha um aumento de indivíduos não curados que formam censurados;

- um aumento em $\sigma_{V}^{2}$ (de 0,5 para 1) produza uma maior variabilidade entre as clínicas, acarretando estimativas não tão boas quanto as obtidas para valores menores de $\sigma_{V}^{2}$;

- o aumento no número de indivíduos por clínica (de 15 para 50) melhore a precisão dos resultados assintóticos.

\subsection{Simulação - abordagem bayesiana}

Em um primeiro momento foi feito um estudo de simulação com a utilização do programa Winbugs, versão 1.4. Os comandos utilizados encontram-se no apêndice A.2. Os dados utilizados foram gerados com a utilização do programa R e encontram-se no apêndice A.1.

Os dados foram compilados sempre a partir de três cadeias iniciadas em valores distintos, com 30 mil iterações cada.

\subsubsection{Caso $n_{k}=15$}

Os resultados obtidos estão na Tabela 3.1, em que a terceira coluna apresenta a média das estimativas obtidas nas 50 simulações. Na quarta coluna está o desvio padrão calculado a partir dos 50 valores de estimativas de cada parâmetro. E, a quinta coluna contém a média dos desvios-padrão encontrados em cada uma das 50 simulações. 
Tabela 3.1: Resultado de 50 simulações via Metropolis-Hastings para $n_{k}=15$

\begin{tabular}{|c|c|c|c|c|}
\hline \multicolumn{5}{|c|}{ Grupo 1: $\left(\alpha=1 ; \sigma_{V}^{2}=0,5 ;\right.$ Censura $\left.=500 ; \beta_{1}=-0,5 ; \gamma_{0}=0 ; \gamma_{1}=0,5\right)$} \\
\hline Parâmetro & Valor verdadeiro & Média da estimativa & DP da estimativa & Média dos DPs \\
\hline$\alpha$ & 1 & 1,0013 & 0,0729 & 0,0758 \\
\hline$\beta_{1}$ & $-0,5$ & $-0,4761$ & 0,2584 & 0,2280 \\
\hline$\gamma_{0}$ & 0 & 0,0298 & 0,2295 & 0,1913 \\
\hline$\gamma_{1}$ & 0,5 & 0,5203 & 0,2438 & 0,2326 \\
\hline$\sigma_{V}^{2}$ & 0,5 & 0,4018 & 0,1689 & 0,1878 \\
\hline \multicolumn{5}{|c|}{ Grupo 2: $\left(\alpha=1,3 ; \sigma_{V}^{2}=0,5 ;\right.$ Censura $\left.=500 ; \beta_{1}=-0,5 ; \gamma_{0}=0 ; \gamma_{1}=0,5\right)$} \\
\hline Parâmetro & Valor verdadeiro & Média da estimativa & DP da estimativa & Média dos DPs \\
\hline$\alpha$ & 1,3 & 1,2782 & 0,0902 & 0,0988 \\
\hline$\beta_{1}$ & $-0,5$ & $-0,6180$ & 0,2329 & 0,2309 \\
\hline$\gamma_{0}$ & 0 & $-0,0323$ & 0,2763 & 0,2019 \\
\hline$\gamma_{1}$ & 0,5 & 0,4625 & 0,2440 & 0,2350 \\
\hline$\sigma_{V}^{2}$ & 0,5 & 0,4764 & 0,2361 & 0,2234 \\
\hline \multicolumn{5}{|c|}{ Grupo 3: $\left(\alpha=1 ; \sigma_{V}^{2}=1 ;\right.$ Censura $\left.=500 ; \beta_{1}=-0,5 ; \gamma_{0}=0 ; \gamma_{1}=0,5\right)$} \\
\hline Parâmetro & Valor verdadeiro & Média da estimativa & DP da estimativa & Média dos DPs \\
\hline$\alpha$ & 1 & 0,9914 & 0,0803 & 0,0755 \\
\hline$\beta_{1}$ & $-0,5$ & $-0,4312$ & 0,2756 & 0,2434 \\
\hline$\gamma_{0}$ & 0 & 0,0974 & 0,3264 & 0,2350 \\
\hline$\gamma_{1}$ & 0,5 & 0,4153 & 0,2657 & 0,2452 \\
\hline$\sigma_{V}^{2}$ & 1 & 0,7686 & 0,3940 & 0,3497 \\
\hline \multicolumn{5}{|c|}{ Grupo 4: $\left(\alpha=1 ; \sigma_{V}^{2}=0,5 ;\right.$ Censura $\left.=300 ; \beta_{1}=-0,5 ; \gamma_{0}=0 ; \gamma_{1}=0,5\right)$} \\
\hline Parâmetro & Valor verdadeiro & Média da estimativa & DP da estimativa & Média dos DPs \\
\hline$\alpha$ & 1 & 0,9918 & 0,0712 & 0,0756 \\
\hline$\beta_{1}$ & $-0,5$ & $-0,5121$ & 0,2570 & 0,2255 \\
\hline$\gamma_{0}$ & 0 & 0,0049 & 0,1872 & 0,1912 \\
\hline$\gamma_{1}$ & 0,5 & 0,4752 & 0,2143 & 0,2310 \\
\hline$\sigma_{V}^{2}$ & 0,5 & 0,3985 & 0,1367 & 0,1878 \\
\hline \multicolumn{5}{|c|}{ Grupo 5: $\left(\alpha=1 ; \sigma_{V}^{2}=0,5 ;\right.$ Censura $\left.=500 ; \beta_{1}=-0,5 ; \gamma_{0}=-0,5 ; \gamma_{1}=0,5\right)$} \\
\hline Parâmetro & Valor verdadeiro & Média da estimativa & DP da estimativa & Média dos DPs \\
\hline$\alpha$ & 1 & 1,0124 & 0,0828 & 0,0878 \\
\hline$\beta_{1}$ & $-0,5$ & $-0,4360$ & 0,2357 & 0,2249 \\
\hline$\gamma_{0}$ & $-0,5$ & $-0,5155$ & 0,2291 & 0,2007 \\
\hline$\gamma_{1}$ & 0,5 & 0,4561 & 0,2149 & 0,2475 \\
\hline$\sigma_{V}^{2}$ & 0,5 & 0,3446 & 0,1114 & 0,1720 \\
\hline \multicolumn{5}{|c|}{ Grupo 6: $\left(\alpha=1,3 ; \sigma_{V}^{2}=0,5 ;\right.$ Censura $\left.=500 ; \beta_{1}=-0,5 ; \gamma_{0}=-0,5 ; \gamma_{1}=0,5\right)$} \\
\hline Parâmetro & Valor verdadeiro & Média da estimativa & DP da estimativa & Média dos DPs \\
\hline$\alpha$ & 1,3 & 1,3221 & 0,1337 & 0,1140 \\
\hline$\beta_{1}$ & $-0,5$ & $-0,5985$ & 0,2450 & 0,2278 \\
\hline$\gamma_{0}$ & $-0,5$ & $-0,4857$ & 0,2461 & 0,1997 \\
\hline$\gamma_{1}$ & 0,5 & 0,4587 & 0,2358 & 0,2466 \\
\hline$\sigma_{V}^{2}$ & 0,5 & 0,3500 & 0,1774 & 0,1710 \\
\hline
\end{tabular}




\begin{tabular}{|c|c|c|c|c|}
\hline \multicolumn{5}{|c|}{ Grupo 7: $\left(\alpha=1 ; \sigma_{V}^{2}=1 ;\right.$ Censura $\left.=500 ; \beta_{1}=-0,5 ; \gamma_{0}=-0,5 ; \gamma_{1}=0,5\right)$} \\
\hline Parâmetro & Valor verdadeiro & Média da estimativa & DP da estimativa & Média dos DPs \\
\hline$\alpha$ & 1 & 1,0123 & 0,0960 & 0,0860 \\
\hline$\beta_{1}$ & $-0,5$ & $-0,4852$ & 0,2662 & 0,2378 \\
\hline$\gamma_{0}$ & $-0,5$ & $-0,3757$ & 0,3325 & 0,2332 \\
\hline$\gamma_{1}$ & 0,5 & 0,4674 & 0,2294 & 0,2522 \\
\hline$\sigma_{V}^{2}$ & 1 & 0,6556 & 0,3618 & 0,3116 \\
\hline \multicolumn{5}{|c|}{ Grupo 8: $\left(\alpha=1 ; \sigma_{V}^{2}=0,5 ;\right.$ Censura $\left.=300 ; \beta_{1}=-0,5 ; \gamma_{0}=-0,5 ; \gamma_{1}=0,5\right)$} \\
\hline Parâmetro & Valor verdadeiro & Média da estimativa & DP da estimativa & Média dos DPs \\
\hline$\alpha$ & 1 & 1,0148 & 0,0937 & 0,0876 \\
\hline$\beta_{1}$ & $-0,5$ & $-0,4553$ & 0,2364 & 0,2276 \\
\hline$\gamma_{0}$ & $-0,5$ & $-0,4264$ & 0,2709 & 0,2060 \\
\hline$\gamma_{1}$ & 0,5 & 0,4434 & 0,2409 & 0,2461 \\
\hline$\sigma_{V}^{2}$ & 0,5 & 0,4148 & 0,2717 & 0,2006 \\
\hline \multicolumn{5}{|c|}{ Grupo 9: $\left(\alpha=1 ; \sigma_{V}^{2}=0,5 ;\right.$ Censura $\left.=500 ; \beta_{1}=-1 ; \gamma_{0}=-1 ; \gamma_{1}=1\right)$} \\
\hline Parâmetro & Valor verdadeiro & Média da estimativa & DP da estimativa & Média dos DPs \\
\hline$\alpha$ & 1 & 1,0078 & 0,0909 & 0,0958 \\
\hline$\beta_{1}$ & -1 & $-0,9968$ & 0,2373 & 0,2433 \\
\hline$\gamma_{0}$ & -1 & $-0,9794$ & 0,2831 & 0,2279 \\
\hline$\gamma_{1}$ & 1 & 0,9486 & 0,2905 & 0,2679 \\
\hline$\sigma_{V}^{2}$ & 0,5 & 0,3824 & 0,2114 & 0,1956 \\
\hline \multicolumn{5}{|c|}{ Grupo 10: $\left(\alpha=1,3 ; \sigma_{V}^{2}=0,5 ;\right.$ Censura $\left.=500 ; \beta_{1}=-1 ; \gamma_{0}=-1 ; \gamma_{1}=1\right)$} \\
\hline Parâmetro & Valor verdadeiro & Média da estimativa & DP da estimativa & Média dos DPs \\
\hline$\alpha$ & 1,3 & 1,2952 & 0,1154 & 0,1193 \\
\hline$\beta_{1}$ & -1 & $-1,2092$ & 0,2762 & 0,2479 \\
\hline$\gamma_{0}$ & -1 & $-0,8451$ & 0,3201 & $-0,2165$ \\
\hline$\gamma_{1}$ & 1 & 0,9331 & 0,2662 & 0,2609 \\
\hline$\sigma_{V}^{2}$ & 0,5 & 0,3413 & 0,1461 & 0,1728 \\
\hline \multicolumn{5}{|c|}{ Grupo 11: $\left(\alpha=1 ; \sigma_{V}^{2}=1 ;\right.$ Censura $\left.=500 ; \beta_{1}=-1 ; \gamma_{0}=-1 ; \gamma_{1}=1\right)$} \\
\hline Parâmetro & Valor verdadeiro & Média da estimativa & DP da estimativa & Média dos DPs \\
\hline$\alpha$ & 1 & 1,0149 & 0,1164 & 0,0951 \\
\hline$\beta_{1}$ & -1 & $-1,0114$ & 0,2464 & 0,2509 \\
\hline$\gamma_{0}$ & -1 & $-0,9043$ & 0,3185 & 0,2587 \\
\hline$\gamma_{1}$ & 1 & 0,9532 & 0,2435 & 0,2712 \\
\hline$\sigma_{V}^{2}$ & 1 & 0,7230 & 0,5260 & 0,3477 \\
\hline \multicolumn{5}{|c|}{ Grupo 12: $\left(\alpha=1 ; \sigma_{V}^{2}=0,5 ;\right.$ Censura $\left.=300 ; \beta_{1}=-1 ; \gamma_{0}=-1 ; \gamma_{1}=1\right)$} \\
\hline Parâmetro & Valor verdadeiro & Média da estimativa & DP da estimativa & Média dos DPs \\
\hline$\alpha$ & 1 & 1,0280 & 0,0899 & 0,0959 \\
\hline$\beta_{1}$ & -1 & $-0,9455$ & 0,2482 & 0,2383 \\
\hline$\gamma_{0}$ & -1 & $-0,8869$ & 0,3019 & 0,2207 \\
\hline$\gamma_{1}$ & 1 & 0,9310 & 0,3103 & 0,2617 \\
\hline$\sigma_{V}^{2}$ & 0,5 & 0,3598 & 0,1823 & 0,1827 \\
\hline
\end{tabular}

Note que a diferença entre os valores médios estimados e os valores verdadeiros são quase sempre menores do que um desvio-padrão, o que indica que os resultados obtidos via simulação estão adequados. 
As Figuras de 3.1 a 3.3 mostram os histogramas feitos a partir das estimativas calculadas nas 50 simulações, para cada um dos parâmetros.
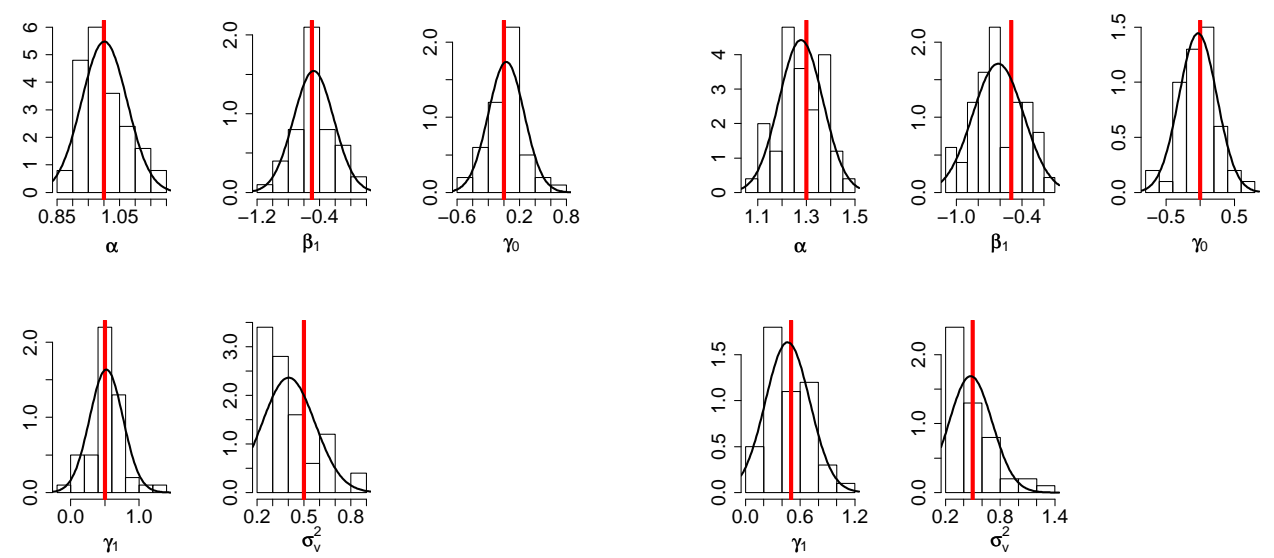

(a) Grupo 1

(b) Grupo 2
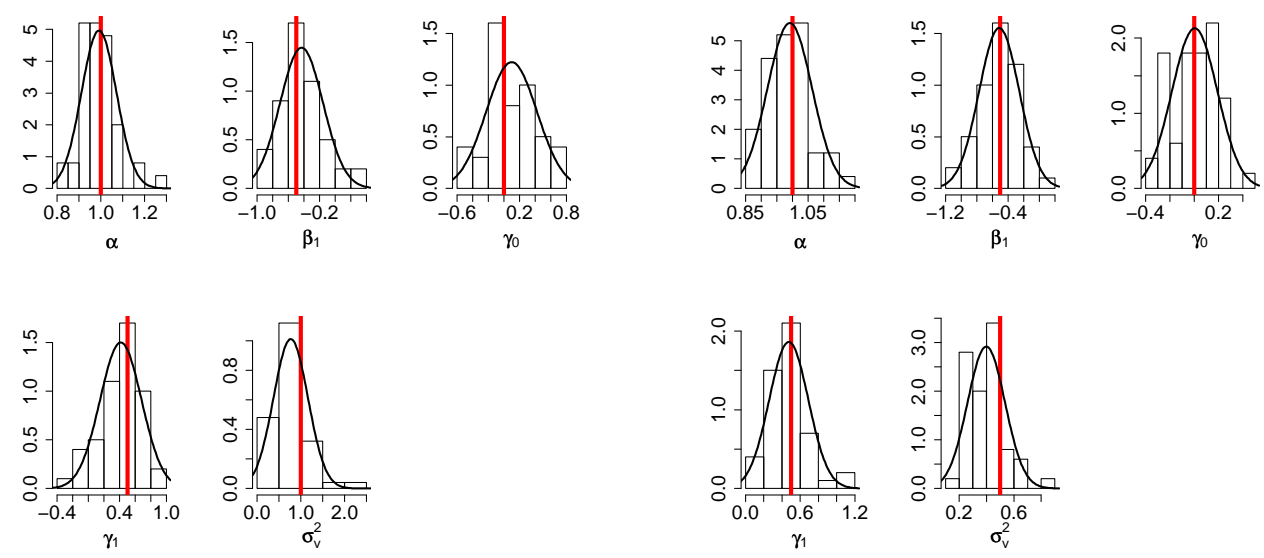

(c) Grupo 3

(d) Grupo 4

Figura 3.1: Histogramas das estimativas dos parâmetros estimados via Metropolis-Hastings $-n_{k}=15-$ parte 1 

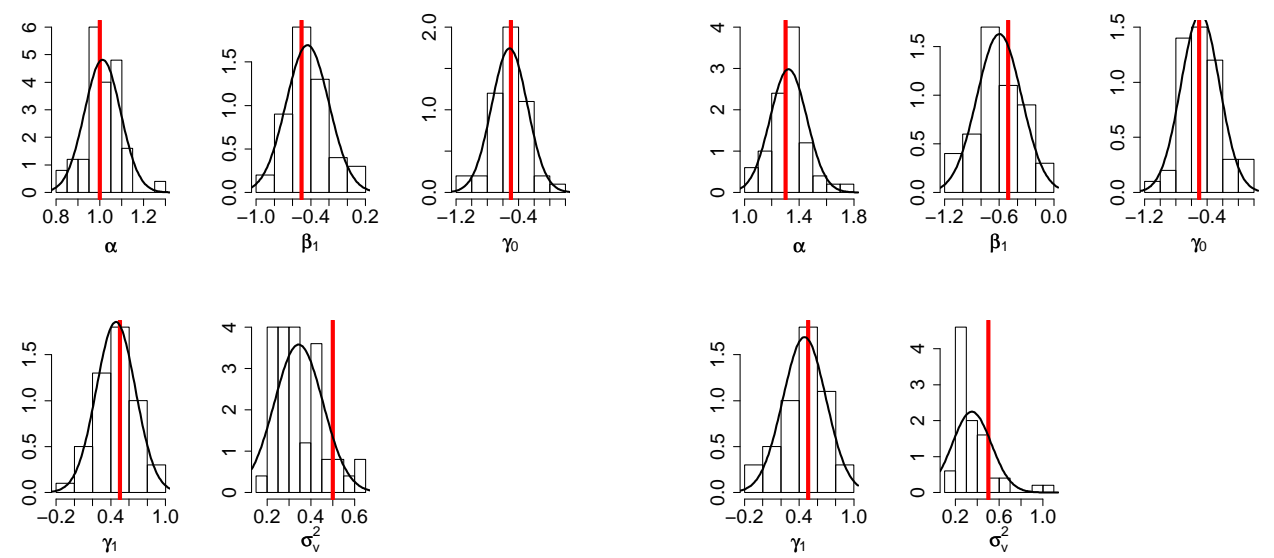

(a) Grupo 5

(b) Grupo 6
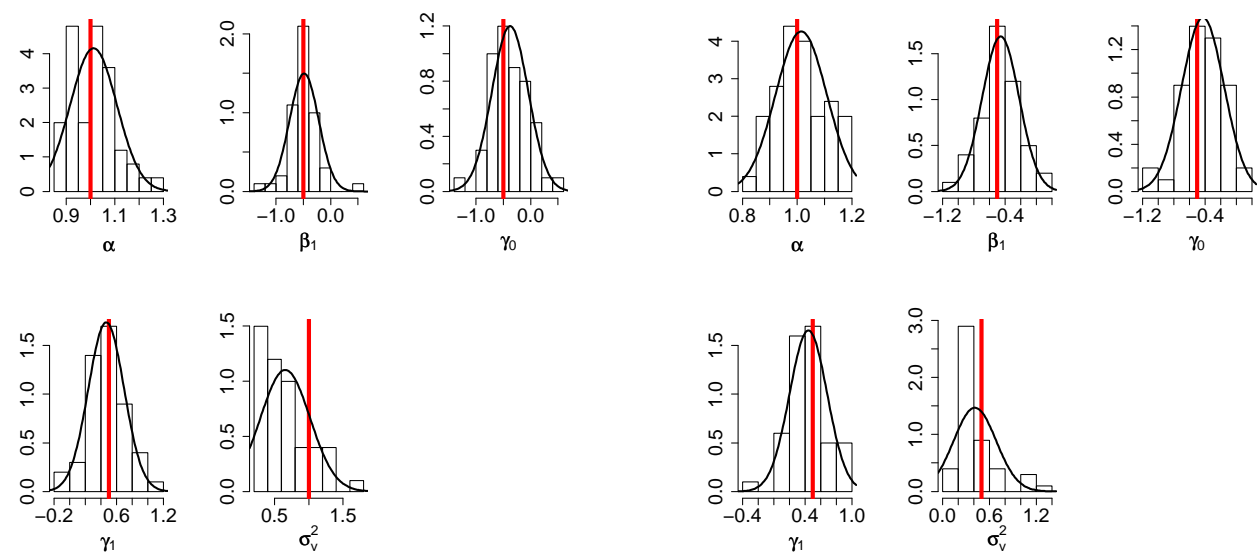

(c) Grupo 7

(d) Grupo 8

Figura 3.2: Histogramas das estimativas dos parâmetros estimados via Metropolis-Hastings $-n_{k}=15-$ parte 2 

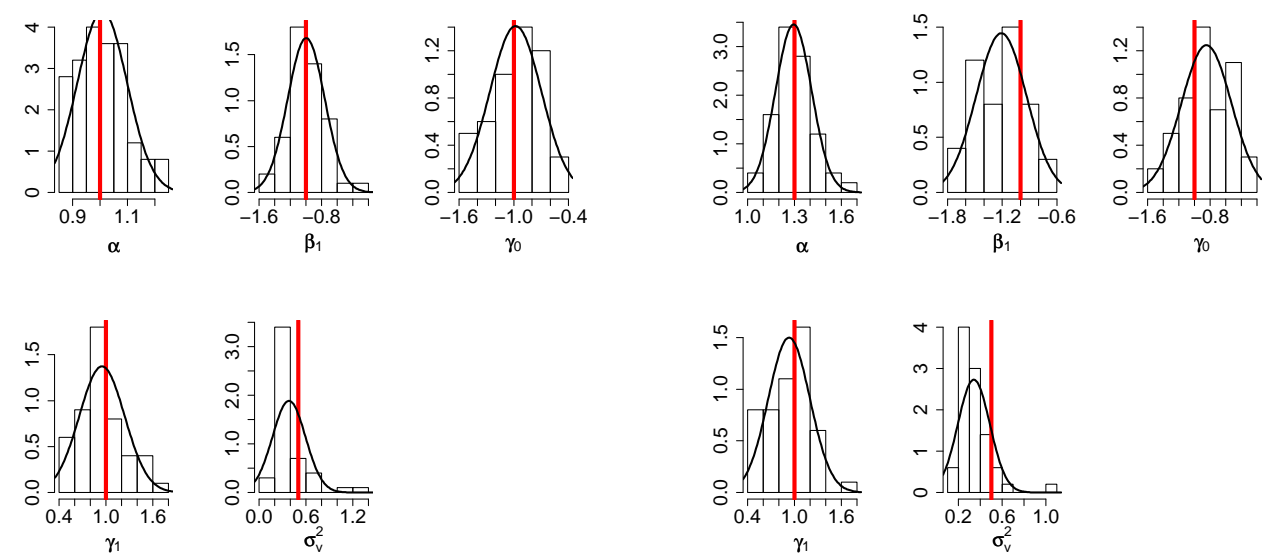

(a) Grupo 9

(b) Grupo 10
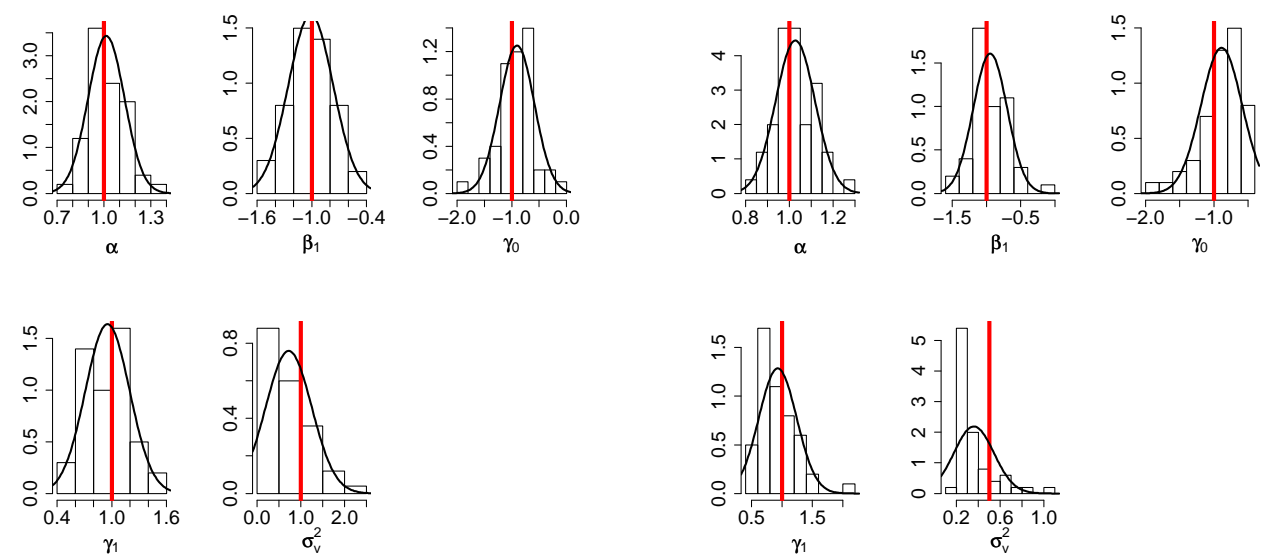

(c) Grupo 11

(d) Grupo 12

Figura 3.3: Histogramas das estimativas dos parâmetros estimados via Metropolis-Hastings $-n_{k}=15-$ parte 3

Estes histogramas indicam que as estimativas obtidas estão em torno dos valores verdadeiros, que estão representados pelas retas verticais. No caso de $\sigma_{V}^{2}$, há um indício de que os valores estão sendo subestimados.

Para se avaliar se a convergência do modelo está adequada, calcula-se os valores de $\sqrt{\hat{R}}$, como definido na seção 2.2. Para isso, considere $m=3$ o número de cadeias utilizadas com valores iniciais distintos, em que cada uma apresenta $2 n=30000$ valores, ou seja, $n=15000$. Foram calculados os valores de $\sqrt{\hat{R}}$ para simulações em cada um dos 12 grupos. Os resultados obtidos encontram-se na Tabela 3.2 . 
Tabela 3.2: Valores de $\sqrt{\hat{R}}-$ caso $n_{k}=15$

\begin{tabular}{ccccccc}
\hline parâmetro & $\mathbf{1}$ & $\mathbf{2}$ & $\mathbf{3}$ & $\mathbf{4}$ & $\mathbf{5}$ & $\mathbf{6}$ \\
\hline$\alpha$ & 1,00156 & 1,00045 & 1,00134 & 1,00086 & 1,00142 & 1,00124 \\
$\beta_{1}$ & 1,00030 & 1,00394 & 1,00410 & 1,00030 & 1,00229 & 1,00013 \\
$\gamma_{0}$ & 1,00158 & 1,00045 & 1,00606 & 1,00292 & 1,00303 & 1,00151 \\
$\gamma_{1}$ & 1,00051 & 1,00228 & 1,00071 & 1,00035 & 1,00345 & 1,00285 \\
$\sigma_{V}^{2}$ & 1,00271 & 1,00074 & 1,00137 & 1,00277 & 1,00178 & 1,00201 \\
\hline \multicolumn{7}{c}{} \\
parâmetro & $\mathbf{7}$ & $\mathbf{8}$ & $\mathbf{9}$ & $\mathbf{1 0}$ & $\mathbf{1 1}$ & $\mathbf{1 2}$ \\
\hline$\alpha$ & 1,00106 & 1,00026 & 1,00076 & 1,00158 & 1,00101 & 1,00174 \\
$\beta_{1}$ & 1,00115 & 1,00164 & 1,00052 & 1,00017 & 1,00063 & 1,00094 \\
$\gamma_{0}$ & 1,00176 & 1,00141 & 1,00419 & 1,00145 & 1,00148 & 1,00139 \\
$\gamma_{1}$ & 1,00050 & 1,00104 & 1,00036 & 1,00026 & 1,00142 & 1,00070 \\
$\sigma_{V}^{2}$ & 1,00196 & 1,00104 & 1,00668 & 1,00245 & 1,00034 & 1,00365 \\
\hline
\end{tabular}

É perceptível que todos os valores estão bem próximos a 1 para todas as estimativas de interesse indicando que houve convergência da cadeia e que foi atingida a distribuição de cada estimador.

\subsubsection{Caso $n_{k}=50$}

Os resultados obtidos estão na Tabela 3.3, em que a terceira coluna apresenta a média das estimativas obtidas nas 50 simulações. Na quarta coluna está o desvio padrão calculado a partir dos 50 valores de estimativas de cada parâmetro. E, a quinta coluna contém a média dos desvios-padrão encontrados em cada uma das 50 simulações. 
Tabela 3.3: Resultado de 50 simulações via Metropolis-Hastings para $n_{k}=50$

\begin{tabular}{|c|c|c|c|c|}
\hline \multicolumn{5}{|c|}{ Grupo 1: $\left(\alpha=1 ; \sigma_{V}^{2}=0,5 ;\right.$ Censura $\left.=500 ; \beta_{1}=-0,5 ; \gamma_{0}=0 ; \gamma_{1}=0,5\right)$} \\
\hline Parâmetro & Valor verdadeiro & Média da estimativa & DP da estimativa & Média dos DPs \\
\hline$\alpha$ & 1 & 0.9980 & 0.0386 & 0.0404 \\
\hline$\beta_{1}$ & -0.5 & -0.5076 & 0.1101 & 0.1241 \\
\hline$\gamma_{0}$ & 0 & 0.0465 & 0.1894 & 0.1812 \\
\hline$\gamma_{1}$ & 0.5 & 0.4840 & 0.1052 & 0.1262 \\
\hline$\sigma_{V}^{2}$ & 0.5 & 0.5675 & 0.2428 & 0.2169 \\
\hline \multicolumn{5}{|c|}{ Grupo 2: $\left(\alpha=1,3 ; \sigma_{V}^{2}=0,5 ;\right.$ Censura $\left.=500 ; \beta_{1}=-0,5 ; \gamma_{0}=0 ; \gamma_{1}=0,5\right)$} \\
\hline Parâmetro & Valor verdadeiro & Média da estimativa & DP da estimativa & Média dos DPs \\
\hline$\alpha$ & 1.3 & 1.3011 & 0.0549 & 0.0534 \\
\hline$\beta_{1}$ & -0.5 & -0.6273 & 0.1299 & 0.1233 \\
\hline$\gamma_{0}$ & 0 & -0.0123 & 0.2333 & 0.1743 \\
\hline$\gamma_{1}$ & 0.5 & 0.5099 & 0.1239 & 0.1255 \\
\hline$\sigma_{V}^{2}$ & 0.5 & 0.5215 & 0.2317 & 0.1991 \\
\hline \multicolumn{5}{|c|}{ Grupo 3: $\left(\alpha=1 ; \sigma_{V}^{2}=1 ;\right.$ Censura $\left.=500 ; \beta_{1}=-0,5 ; \gamma_{0}=0 ; \gamma_{1}=0,5\right)$} \\
\hline Parâmetro & Valor verdadeiro & Média da estimativa & DP da estimativa & Média dos DPs \\
\hline$\alpha$ & 1 & 0.9923 & 0.0385 & 0.0410 \\
\hline$\beta_{1}$ & -0.5 & -0.4895 & 0.1489 & 0.1304 \\
\hline$\gamma_{0}$ & 0 & 0.0201 & 0.2876 & 0.2299 \\
\hline$\gamma_{1}$ & 0.5 & 0.4829 & 0.1293 & 0.1314 \\
\hline$\sigma_{V}^{2}$ & 1 & 1.0096 & 0.4277 & 0.3798 \\
\hline \multicolumn{5}{|c|}{ Grupo 4: $\left(\alpha=1 ; \sigma_{V}^{2}=0,5 ;\right.$ Censura $\left.=300 ; \beta_{1}=-0,5 ; \gamma_{0}=0 ; \gamma_{1}=0,5\right)$} \\
\hline Parâmetro & Valor verdadeiro & Média da estimativa & DP da estimativa & Média dos DPs \\
\hline$\alpha$ & 1 & 1.0043 & 0.0427 & 0.0414 \\
\hline$\beta_{1}$ & -0.5 & -0.5092 & 0.1489 & 0.1212 \\
\hline$\gamma_{0}$ & 0 & -0.0155 & 0.2228 & 0.1659 \\
\hline$\gamma_{1}$ & 0.5 & 0.4956 & 0.1064 & 0.1246 \\
\hline$\sigma_{V}^{2}$ & 0.5 & 0.4647 & 0.1786 & 0.1784 \\
\hline \multicolumn{5}{|c|}{ Grupo 5: $\left(\alpha=1 ; \sigma_{V}^{2}=0,5 ;\right.$ Censura $\left.=500 ; \beta_{1}=-0,5 ; \gamma_{0}=-0,5 ; \gamma_{1}=0,5\right)$} \\
\hline Parâmetro & Valor verdadeiro & Média da estimativa & DP da estimativa & Média dos DPs \\
\hline$\alpha$ & 1 & 0.9922 & 0.0444 & 0.0467 \\
\hline$\beta_{1}$ & -0.5 & -0.4811 & 0.1373 & 0.1229 \\
\hline$\gamma_{0}$ & -0.5 & -0.4965 & 0.2411 & 0.1806 \\
\hline$\gamma_{1}$ & 0.5 & 0.5050 & 0.1182 & 0.1335 \\
\hline$\sigma_{V}^{2}$ & 0.5 & 0.5314 & 0.2238 & 0.2094 \\
\hline \multicolumn{5}{|c|}{ Grupo 6: $\left(\alpha=1,3 ; \sigma_{V}^{2}=0,5 ;\right.$ Censura $\left.=500 ; \beta_{1}=-0,5 ; \gamma_{0}=-0,5 ; \gamma_{1}=0,5\right)$} \\
\hline Parâmetro & Valor verdadeiro & Média da estimativa & DP da estimativa & Média dos DPs \\
\hline$\alpha$ & 1.3 & 1.3055 & 0.0692 & 0.0611 \\
\hline$\beta_{1}$ & -0.5 & -0.6222 & 0.1050 & 0.1239 \\
\hline$\gamma_{0}$ & -0.5 & -0.5114 & 0.2547 & 0.1783 \\
\hline$\gamma_{1}$ & 0.5 & 0.5190 & 0.1424 & 0.1341 \\
\hline$\sigma_{V}^{2}$ & 0.5 & 0.5084 & 0.2183 & 0.1994 \\
\hline
\end{tabular}


28CAPÍTULO 3. ESTUDOS DE SIMULAÇÃO - MODELO DE CHEN-IBRAHIM-SINHA COM EFEITOS A

\begin{tabular}{|c|c|c|c|c|}
\hline \multicolumn{5}{|c|}{ Grupo 7: $\left(\alpha=1 ; \sigma_{V}^{2}=1 ;\right.$ Censura $\left.=500 ; \beta_{1}=-0,5 ; \gamma_{0}=-0,5 ; \gamma_{1}=0,5\right)$} \\
\hline Parâmetro & Valor verdadeiro & Média da estimativa & DP da estimativa & Média dos DPs \\
\hline$\alpha$ & 1 & 0.9955 & 0.0464 & 0.0452 \\
\hline$\beta_{1}$ & -0.5 & -0.4896 & 0.1391 & 0.1287 \\
\hline$\gamma_{0}$ & -0.5 & -0.3946 & 0.3294 & 0.2287 \\
\hline$\gamma_{1}$ & 0.5 & 0.4964 & 0.1163 & 0.1355 \\
\hline$\sigma_{V}^{2}$ & 1 & 0.9379 & 0.4815 & 0.3619 \\
\hline \multicolumn{5}{|c|}{ Grupo 8: $\left(\alpha=1 ; \sigma_{V}^{2}=0,5 ;\right.$ Censura $\left.=300 ; \beta_{1}=-0,5 ; \gamma_{0}=-0,5 ; \gamma_{1}=0,5\right)$} \\
\hline Parâmetro & Valor verdadeiro & Média da estimativa & DP da estimativa & Média dos DPs \\
\hline$\alpha$ & 1 & 0.9990 & 0.0495 & 0.0465 \\
\hline$\beta_{1}$ & -0.5 & -0.5196 & 0.1332 & 0.1224 \\
\hline$\gamma_{0}$ & -0.5 & -0.4687 & 0.2476 & 0.1755 \\
\hline$\gamma_{1}$ & 0.5 & 0.5060 & 0.1156 & 0.1324 \\
\hline$\sigma_{V}^{2}$ & 0.5 & 0.4969 & 0.2288 & 0.1945 \\
\hline \multicolumn{5}{|c|}{ Grupo 9: $\left(\alpha=1 ; \sigma_{V}^{2}=0,5 ;\right.$ Censura $\left.=500 ; \beta_{1}=-1 ; \gamma_{0}=-1 ; \gamma_{1}=1\right)$} \\
\hline Parâmetro & Valor verdadeiro & Média da estimativa & DP da estimativa & Média dos DPs \\
\hline$\alpha$ & 1 & 0.9982 & 0.0579 & 0.0513 \\
\hline$\beta_{1}$ & -1 & -0.9847 & 0.1273 & 0.1305 \\
\hline$\gamma_{0}$ & -1 & -0.9924 & 0.2204 & 0.1770 \\
\hline$\gamma_{1}$ & 1 & 1.0009 & 0.1332 & 0.1435 \\
\hline$\sigma_{V}^{2}$ & 0.5 & 0.4385 & 0.1959 & 0.1781 \\
\hline \multicolumn{5}{|c|}{ Grupo 10: $\left(\alpha=1,3 ; \sigma_{V}^{2}=0,5 ;\right.$ Censura $\left.=500 ; \beta_{1}=-1 ; \gamma_{0}=-1 ; \gamma_{1}=1\right)$} \\
\hline Parâmetro & Valor verdadeiro & Média da estimativa & DP da estimativa & Média dos DPs \\
\hline$\alpha$ & 1.3 & 1.3085 & 0.0880 & 0.0682 \\
\hline$\beta_{1}$ & -1 & -1.2937 & 0.1781 & 0.1388 \\
\hline$\gamma_{0}$ & -1 & -1.0285 & 0.2445 & -0.1861 \\
\hline$\gamma_{1}$ & 1 & 1.0057 & 0.1617 & 0.1450 \\
\hline$\sigma_{V}^{2}$ & 0.5 & 0.5046 & 0.2381 & 0.2031 \\
\hline \multicolumn{5}{|c|}{ Grupo 11: $\left(\alpha=1 ; \sigma_{V}^{2}=1 ;\right.$ Censura $\left.=500 ; \beta_{1}=-1 ; \gamma_{0}=-1 ; \gamma_{1}=1\right)$} \\
\hline Parâmetro & Valor verdadeiro & Média da estimativa & DP da estimativa & Média dos DPs \\
\hline$\alpha$ & 1 & 1.0063 & 0.0493 & 0.0517 \\
\hline$\beta_{1}$ & -1 & -0.9732 & 0.1195 & 0.1356 \\
\hline$\gamma_{0}$ & -1 & -1.0086 & 0.3214 & 0.2327 \\
\hline$\gamma_{1}$ & 1 & 0.9694 & 0.1340 & 0.1476 \\
\hline$\sigma_{V}^{2}$ & 1 & 0.8984 & 0.4011 & 0.3593 \\
\hline \multicolumn{5}{|c|}{ Grupo 12: $\left(\alpha=1 ; \sigma_{V}^{2}=0,5 ;\right.$ Censura $\left.=300 ; \beta_{1}=-1 ; \gamma_{0}=-1 ; \gamma_{1}=1\right)$} \\
\hline Parâmetro & Valor verdadeiro & Média da estimativa & DP da estimativa & Média dos DPs \\
\hline$\alpha$ & 1 & 1.0063 & 0.0511 & 0.0528 \\
\hline$\beta_{1}$ & -1 & -0.9977 & 0.1368 & 0.1317 \\
\hline$\gamma_{0}$ & -1 & -1.0350 & 0.2439 & 0.1809 \\
\hline$\gamma_{1}$ & 1 & 0.9932 & 0.1480 & 0.1458 \\
\hline$\sigma_{V}^{2}$ & 0.5 & 0.4615 & 0.2147 & 0.1889 \\
\hline
\end{tabular}

Note que a diferença entre as estimativas médias estimados e os valores verdadeiros são menores do que um desvio-padrão, em quase todos os casos, o que indica que os resultados obtidos via simulação estão bons. 
Note ainda que as estimativas obtidas para este caso $n_{k}=50$ estão mais próximos dos valores verdadeiros do que no caso $n_{k}=15$, ou seja, o fato de se ter mais indivíduos em cada clínica faz com que as estimativas fiquem mais acuradas.

As Figuras de 3.4 a 3.6 mostram os histogramas feitos a partir das estimativas calculadas nas 50 simulações, para cada um dos parâmetros.
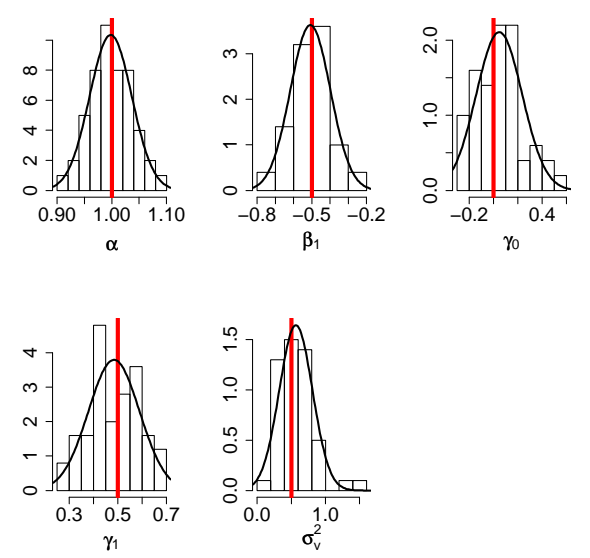

(a) Grupo 1
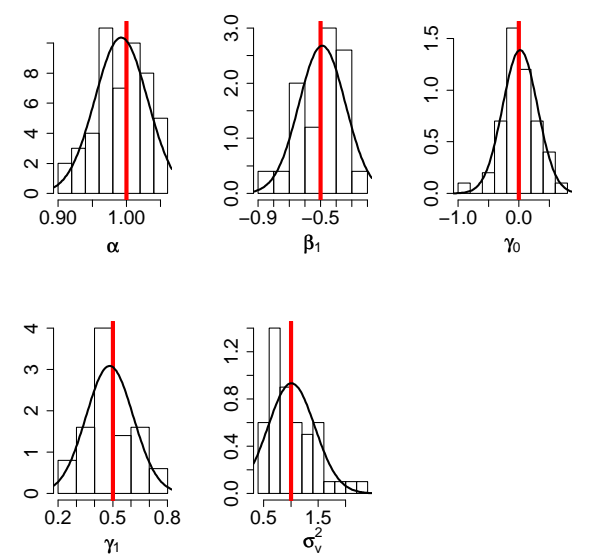

(c) Grupo 3
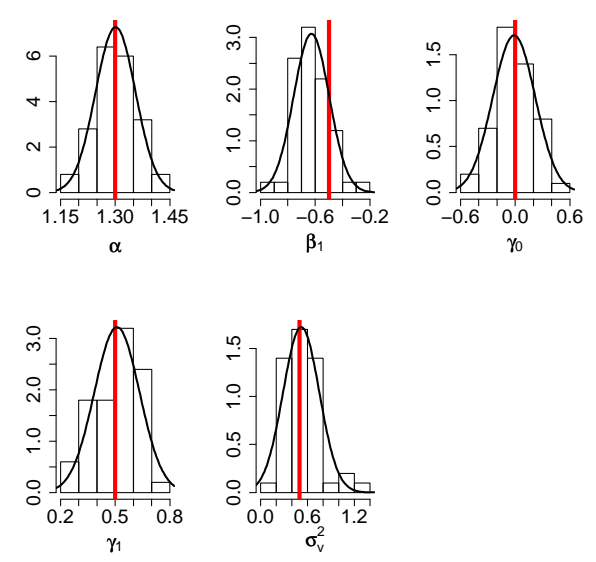

(b) Grupo 2
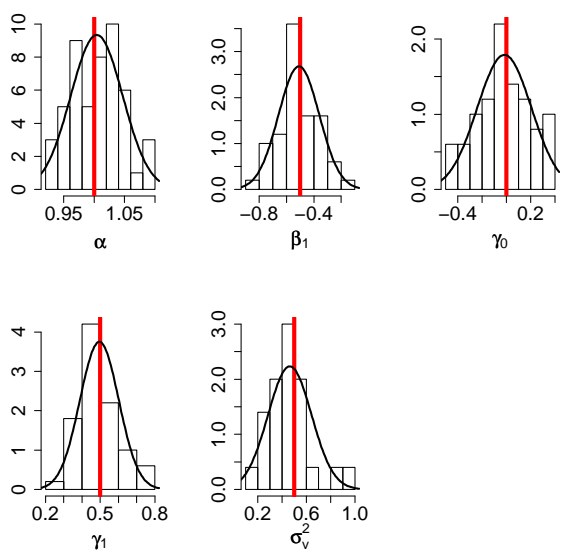

(d) Grupo 4

Figura 3.4: Histogramas das estimativas dos parâmetros estimados via Metropolis-Hastings $-n_{k}=50-$ parte 1 

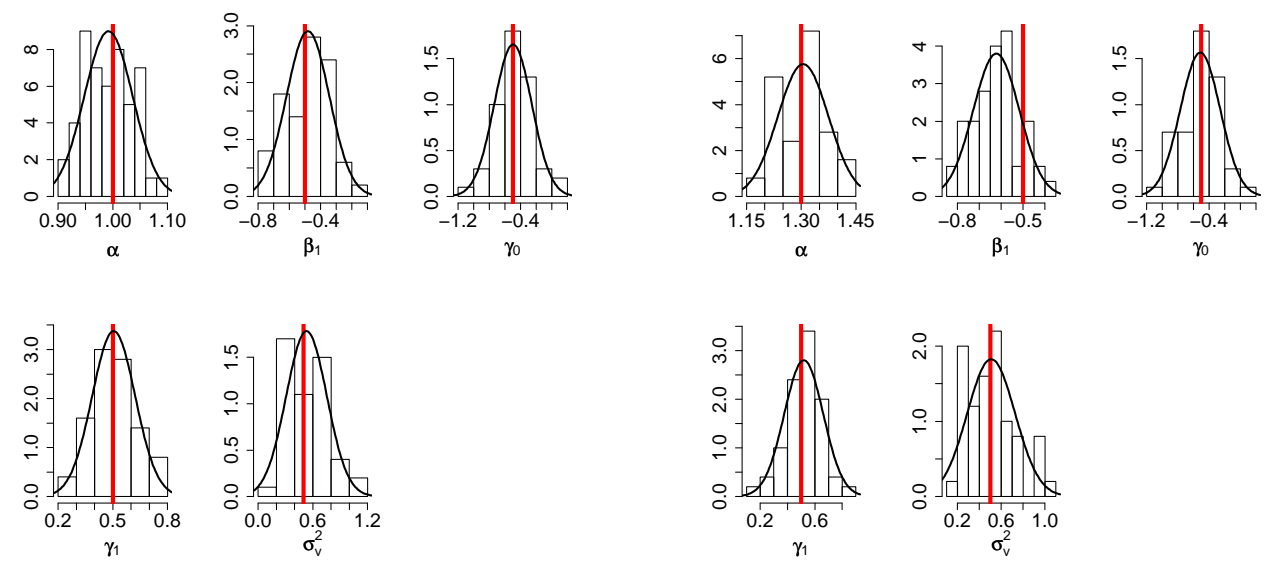

(a) Grupo 5

(b) Grupo 6
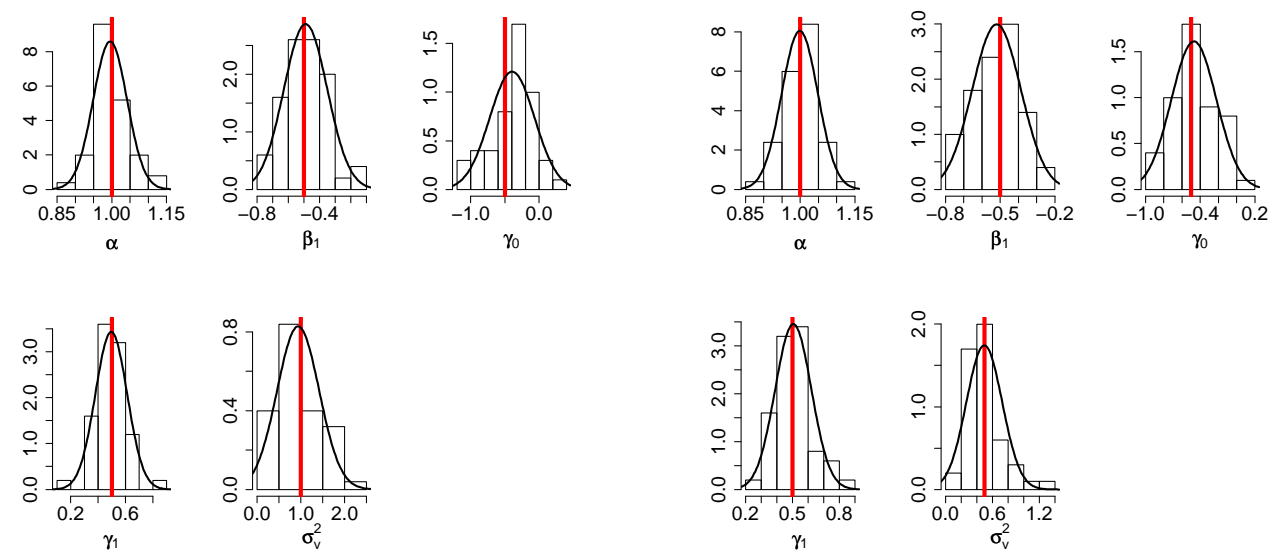

(c) Grupo 7

(d) Grupo 8

Figura 3.5: Histogramas das estimativas dos parâmetros estimados via Metropolis-Hastings $-n_{k}=50-$ parte 2 

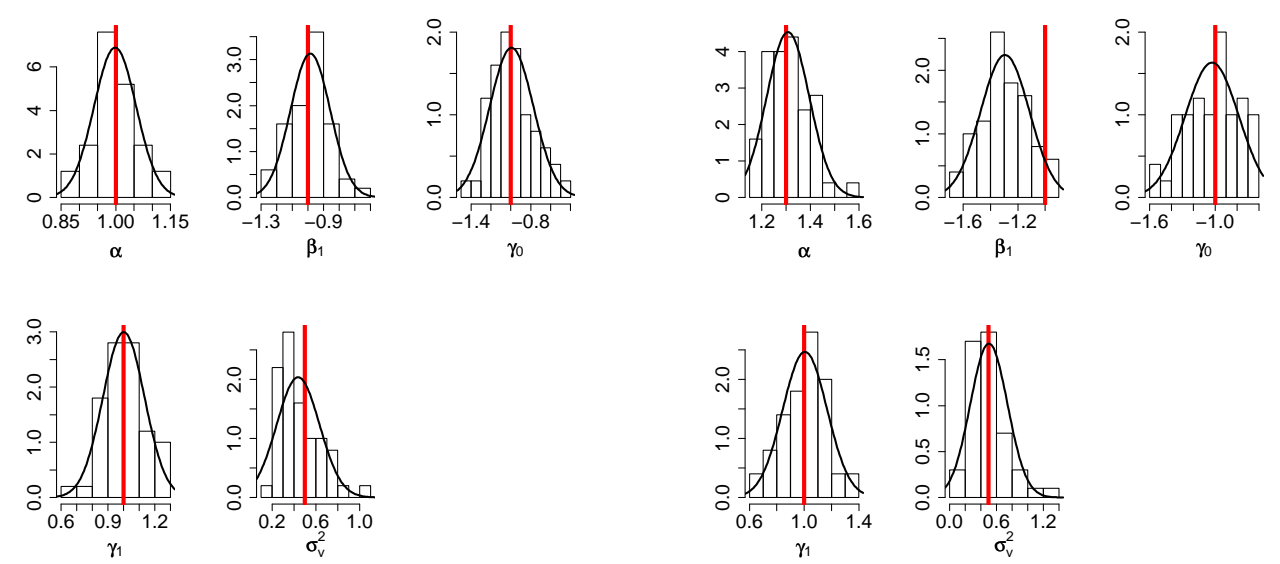

(a) Grupo 9

(b) Grupo 10
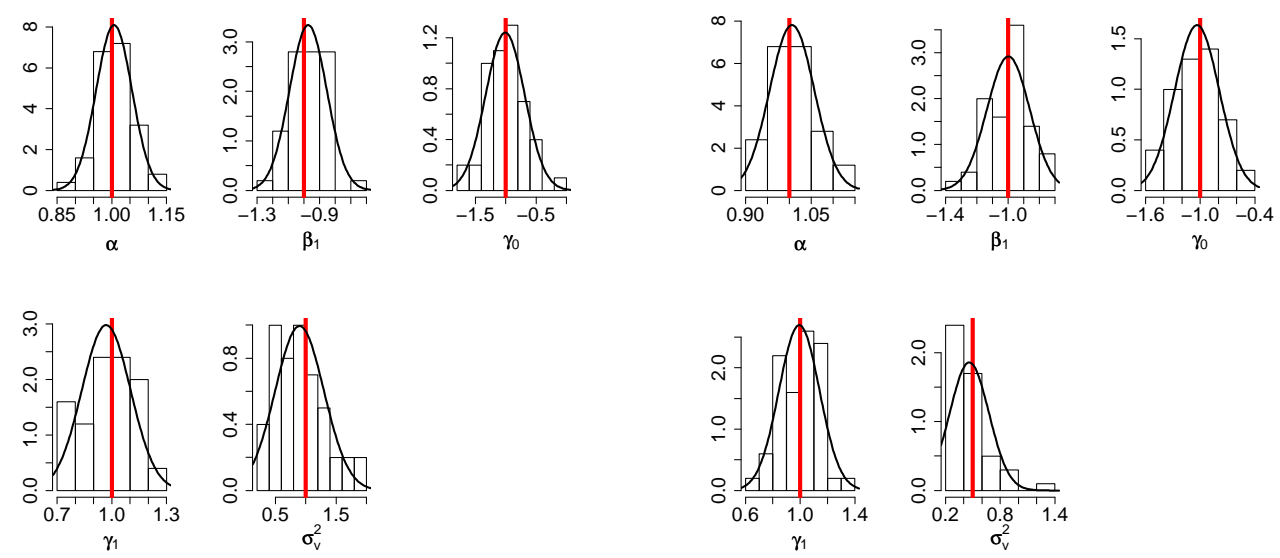

(c) Grupo 11

(d) Grupo 12

Figura 3.6: Histogramas das estimativas dos parâmetros estimados via Metropolis-Hastings $-n_{k}=50-$ parte 3

Estes histogramas indicam que as estimativas obtidas estão em torno dos valores verdadeiros, representados pelas retas verticais. Indicam também que as estimativas não estão muito longe da normalidade assintótica.

O histograma do grupo 10, aparentemente, não está muito bom para o parâmetro $\beta_{1}$, mas a estimativa média difere do valor verdadeiro $(|-1,2937-(-1)|=0,2937)$ por menos de dois desviospadrão $(2 * 0,1781=0,3562)$, o que, portanto, não é nenhum grande problema.

Para se avaliar se a convergência do modelo está adequada, calcula-se os valores de $\sqrt{\hat{R}}$, como definido na seção 2.2. Para isso, considere $m=3$ o número de cadeias utilizadas com valores iniciais distintos, em que cada uma apresenta $2 n=30000$ valores, ou seja, $n=15000$. Foram calculados os valores de $\sqrt{\hat{R}}$ para simulações em cada um dos 12 grupos. Os resultados obtidos encontram-se na Tabela 3.4. 
Tabela 3.4: Valores de $\sqrt{\hat{R}}-$ caso $n_{k}=50$

\begin{tabular}{ccccccc}
\hline parâmetro & $\mathbf{1}$ & $\mathbf{2}$ & $\mathbf{3}$ & $\mathbf{4}$ & $\mathbf{5}$ & $\mathbf{6}$ \\
\hline$\alpha$ & 1,00033 & 1,00018 & 1,00002 & 1,00000 & 0,99998 & 1,00002 \\
$\beta_{1}$ & 1,00083 & 1,00140 & 1,00040 & 1,00005 & 1,00018 & 1,00008 \\
$\gamma_{0}$ & 1,00674 & 1,01705 & 1,01473 & 1,01240 & 1,00145 & 1,00542 \\
$\gamma_{1}$ & 1,00152 & 1,00082 & 1,00055 & 1,00034 & 1,00042 & 1,00131 \\
$\sigma_{V}^{2}$ & 1,00065 & 1,00036 & 1,00132 & 1,00050 & 1,00011 & 1,00087 \\
\hline \multicolumn{7}{c}{} \\
parâmetro & $\mathbf{7}$ & $\mathbf{8}$ & $\mathbf{9}$ & $\mathbf{1 0}$ & $\mathbf{1 1}$ & $\mathbf{1 2}$ \\
\hline$\alpha$ & 1,00006 & 1,00026 & 1,00011 & 1,00010 & 1,00007 & 1,00012 \\
$\beta_{1}$ & 1,00040 & 1,00060 & 1,00061 & 1,00029 & 1,00070 & 1,00028 \\
$\gamma_{0}$ & 1,01302 & 1,00234 & 1,00499 & 1,00240 & 1,01053 & 1,00788 \\
$\gamma_{1}$ & 1,00023 & 1,00200 & 1,00137 & 1,00059 & 1,00244 & 1,00007 \\
$\sigma_{V}^{2}$ & 1,00069 & 1,00026 & 1,00012 & 1,00023 & 1,00090 & 1,00064 \\
\hline
\end{tabular}

É perceptível que todos os valores estão bem próximos a 1 para todas as estimativas de interesse indicando que houve convergência da cadeia e que foi atingida a distribuição de cada estimador.

\subsection{Simulação - abordagem clássica}

Um estudo de simulação da parte clássica foi feito com o auxílio do programa Maple, versão 10. Os dados foram gerados com o programa $\mathrm{R}$ e são os mesmos utilizados na seção 3.2. Os comandos utilizados encontram-se no apêndice A.3.

\subsubsection{Caso $n_{k}=15$}

Os resultados obtidos estão na Tabela 3.5, em que a terceira coluna apresenta a média das estimativas obtidas nas 50 simulações. Na quarta coluna está o desvio padrão calculado a partir dos 50 valores de estimativas de cada parâmetro. E, a quinta coluna contém a média dos desvios-padrão encontrados em cada uma das 50 simulações. Estes desvios-padrão foram obtidos pela raiz quadrada dos elementos da diagonal principal da matriz de informação de Fisher. 
Tabela 3.5: Resultado de 50 simulações via REML para $n_{k}=15$

\begin{tabular}{|c|c|c|c|c|}
\hline \multicolumn{5}{|c|}{ Grupo 1: $\left(\alpha=1 ; \sigma_{V}^{2}=0,5 ;\right.$ Censura $\left.=500 ; \beta_{1}=-0,5 ; \gamma_{0}=0 ; \gamma_{1}=0,5\right)$} \\
\hline Parâmetro & Valor verdadeiro & Média da estimativa & DP da estimativa & Média dos DPs \\
\hline$\alpha$ & 1 & 1,0220 & 0,0736 & 0,0768 \\
\hline$\beta_{1}$ & $-0,5$ & $-0,5302$ & 0,2702 & 0,2365 \\
\hline$\gamma_{0}$ & 0 & 0,0356 & 0,2386 & 0,3122 \\
\hline$\gamma_{1}$ & 0,5 & 0,5744 & 0,2566 & 0,2398 \\
\hline$\sigma_{V}^{2}$ & 0,5 & 0,5914 & 0,1775 & 0,1621 \\
\hline \multicolumn{5}{|c|}{ Grupo 2: $\left(\alpha=1,3 ; \sigma_{V}^{2}=0,5 ;\right.$ Censura $\left.=500 ; \beta_{1}=-0,5 ; \gamma_{0}=0 ; \gamma_{1}=0,5\right)$} \\
\hline Parâmetro & Valor verdadeiro & Média da estimativa & DP da estimativa & Média dos DPs \\
\hline$\alpha$ & 1,3 & 1,3052 & 0,0942 & 0,1001 \\
\hline$\beta_{1}$ & $-0,5$ & $-0,6689$ & 0,2402 & 0,2378 \\
\hline$\gamma_{0}$ & 0 & $-0,0288$ & 0,2850 & 0,3138 \\
\hline$\gamma_{1}$ & 0,5 & 0,5084 & 0,2596 & 0,2401 \\
\hline$\sigma_{V}^{2}$ & 0,5 & 0,6691 & 0,2185 & 0,1829 \\
\hline \multicolumn{5}{|c|}{ Grupo 3: $\left(\alpha=1 ; \sigma_{V}^{2}=1 ;\right.$ Censura $\left.=500 ; \beta_{1}=-0,5 ; \gamma_{0}=0 ; \gamma_{1}=0,5\right)$} \\
\hline Parâmetro & Valor verdadeiro & Média da estimativa & DP da estimativa & Média dos DPs \\
\hline$\alpha$ & 1 & 1,0111 & 0,0813 & 0,0759 \\
\hline$\beta_{1}$ & $-0,5$ & $-0,4710$ & 0,2865 & 0,2493 \\
\hline$\gamma_{0}$ & 0 & 0,1150 & 0,3385 & 0,3135 \\
\hline$\gamma_{1}$ & 0,5 & 0,4577 & 0,2824 & 0,2499 \\
\hline$\sigma_{V}^{2}$ & 1 & 0,9118 & 0,2934 & 0,2401 \\
\hline \multicolumn{5}{|c|}{ Grupo 4: $\left(\alpha=1 ; \sigma_{V}^{2}=0,5 ;\right.$ Censura $\left.=300 ; \beta_{1}=-0,5 ; \gamma_{0}=0 ; \gamma_{1}=0,5\right)$} \\
\hline Parâmetro & Valor verdadeiro & Média da estimativa & DP da estimativa & Média dos DPs \\
\hline$\alpha$ & 1 & 1,0125 & 0,0734 & 0,0765 \\
\hline$\beta_{1}$ & $-0,5$ & $-0,5630$ & 0,2640 & 0,2339 \\
\hline$\gamma_{0}$ & 0 & 0,0089 & 0,1917 & 0,3124 \\
\hline$\gamma_{1}$ & 0,5 & 0,5241 & 0,2304 & 0,2377 \\
\hline$\sigma_{V}^{2}$ & 0,5 & 0,5957 & 0,1541 & 0,1640 \\
\hline \multicolumn{5}{|c|}{ Grupo 5: $\left(\alpha=1 ; \sigma_{V}^{2}=0,5 ;\right.$ Censura $\left.=500 ; \beta_{1}=-0,5 ; \gamma_{0}=-0,5 ; \gamma_{1}=0,5\right)$} \\
\hline Parâmetro & Valor verdadeiro & Média da estimativa & DP da estimativa & Média dos DPs \\
\hline$\alpha$ & 1 & 1,0296 & 0,0848 & 0,0889 \\
\hline$\beta_{1}$ & $-0,5$ & $-0,4746$ & 0,2465 & 0,2299 \\
\hline$\gamma_{0}$ & $-0,5$ & $-0,5379$ & 0,2414 & 0,3232 \\
\hline$\gamma_{1}$ & 0,5 & 0,5049 & 0,2296 & 0,2503 \\
\hline$\sigma_{V}^{2}$ & 0,5 & 0,5964 & 0,1392 & 0,1746 \\
\hline \multicolumn{5}{|c|}{ Grupo 6: $\left(\alpha=1,3 ; \sigma_{V}^{2}=0,5 ;\right.$ Censura $\left.=500 ; \beta_{1}=-0,5 ; \gamma_{0}=-0,5 ; \gamma_{1}=0,5\right)$} \\
\hline Parâmetro & Valor verdadeiro & Média da estimativa & DP da estimativa & Média dos DPs \\
\hline$\alpha$ & 1,3 & 1,3467 & 0,1369 & 0,1156 \\
\hline$\beta_{1}$ & $-0,5$ & $-0,6374$ & 0,2518 & 0,2327 \\
\hline$\gamma_{0}$ & $-0,5$ & $-0,4990$ & 0,2537 & 0,3223 \\
\hline$\gamma_{1}$ & 0,5 & 0,5003 & 0,2511 & 0,2497 \\
\hline$\sigma_{V}^{2}$ & 0,5 & 0,5763 & 0,1903 & 0,1675 \\
\hline
\end{tabular}


34CAPÍTULO 3. ESTUDOS DE SIMULAÇÃO - MODELO DE CHEN-IBRAHIM-SINHA COM EFEITOS A

\begin{tabular}{|c|c|c|c|c|}
\hline \multicolumn{5}{|c|}{ Grupo 7: $\left(\alpha=1 ; \sigma_{V}^{2}=1 ;\right.$ Censura $\left.=500 ; \beta_{1}=-0,5 ; \gamma_{0}=-0,5 ; \gamma_{1}=0,5\right)$} \\
\hline Parâmetro & Valor verdadeiro & Média da estimativa & DP da estimativa & Média dos DPs \\
\hline$\alpha$ & 1 & 1,0303 & 0,0987 & 0,0868 \\
\hline$\beta_{1}$ & $-0,5$ & $-0,5235$ & 0,2805 & 0,2428 \\
\hline$\gamma_{0}$ & $-0,5$ & $-0,3825$ & 0,3470 & 0,3221 \\
\hline$\gamma_{1}$ & 0,5 & 0,5103 & 0,2456 & 0,2552 \\
\hline$\sigma_{V}^{2}$ & 1 & 0,8610 & 0,2732 & 0,2362 \\
\hline \multicolumn{5}{|c|}{ Grupo 8: $\left(\alpha=1 ; \sigma_{V}^{2}=0,5 ;\right.$ Censura $\left.=300 ; \beta_{1}=-0,5 ; \gamma_{0}=-0,5 ; \gamma_{1}=0,5\right)$} \\
\hline Parâmetro & Valor verdadeiro & Média da estimativa & DP da estimativa & Média dos DPs \\
\hline$\alpha$ & 1 & 1,0315 & 0,0974 & 0,0887 \\
\hline$\beta_{1}$ & $-0,5$ & $-0,4925$ & 0,2428 & 0,2330 \\
\hline$\gamma_{0}$ & $-0,5$ & $-0,4379$ & 0,2807 & 0,3215 \\
\hline$\gamma_{1}$ & 0,5 & 0,4865 & 0,2521 & 0,2495 \\
\hline$\sigma_{V}^{2}$ & 0,5 & 0,6311 & 0,2436 & 0,1798 \\
\hline \multicolumn{5}{|c|}{ Grupo 9: $\left(\alpha=1 ; \sigma_{V}^{2}=0,5 ;\right.$ Censura $\left.=500 ; \beta_{1}=-1 ; \gamma_{0}=-1 ; \gamma_{1}=1\right)$} \\
\hline Parâmetro & Valor verdadeiro & Média da estimativa & DP da estimativa & Média dos DPs \\
\hline$\alpha$ & 1 & 1,0283 & 0,0942 & 0,0974 \\
\hline$\beta_{1}$ & -1 & $-1,0647$ & 0,2447 & 0,2504 \\
\hline$\gamma_{0}$ & -1 & $-1,0201$ & 0,2927 & 0,3391 \\
\hline$\gamma_{1}$ & 1 & 1,0323 & 0,3174 & 0,2723 \\
\hline$\sigma_{V}^{2}$ & 0,5 & 0,6599 & 0,2032 & 0,1976 \\
\hline \multicolumn{5}{|c|}{ Grupo 10: $\left(\alpha=1,3 ; \sigma_{V}^{2}=0,5 ;\right.$ Censura $\left.=500 ; \beta_{1}=-1 ; \gamma_{0}=-1 ; \gamma_{1}=1\right)$} \\
\hline Parâmetro & Valor verdadeiro & Média da estimativa & DP da estimativa & Média dos DPs \\
\hline$\alpha$ & 1,3 & 1,3235 & 0,1197 & 0,1212 \\
\hline$\beta_{1}$ & -1 & $-1,2813$ & 0,2887 & 0,2548 \\
\hline$\gamma_{0}$ & -1 & $-0,8808$ & 0,3313 & 0,3340 \\
\hline$\gamma_{1}$ & 1 & 1,0142 & 0,2894 & 0,2652 \\
\hline$\sigma_{V}^{2}$ & 0,5 & 0,6123 & 0,1582 & 0,1830 \\
\hline \multicolumn{5}{|c|}{ Grupo 11: $\left(\alpha=1 ; \sigma_{V}^{2}=1 ;\right.$ Censura $\left.=500 ; \beta_{1}=-1 ; \gamma_{0}=-1 ; \gamma_{1}=1\right)$} \\
\hline Parâmetro & Valor verdadeiro & Média da estimativa & DP da estimativa & Média dos DPs \\
\hline$\alpha$ & 1 & 1,0329 & 0,1216 & 0,0962 \\
\hline$\beta_{1}$ & -1 & $-1,0606$ & 0,2655 & 0,2549 \\
\hline$\gamma_{0}$ & -1 & $-0,9178$ & 0,3302 & 0,3372 \\
\hline$\gamma_{1}$ & 1 & 1,0138 & 0,2545 & 0,2731 \\
\hline$\sigma_{V}^{2}$ & 1 & 0,9002 & 0,3870 & 0,2520 \\
\hline \multicolumn{5}{|c|}{ Grupo 12: $\left(\alpha=1 ; \sigma_{V}^{2}=0,5 ;\right.$ Censura $\left.=300 ; \beta_{1}=-1 ; \gamma_{0}=-1 ; \gamma_{1}=1\right)$} \\
\hline Parâmetro & Valor verdadeiro & Média da estimativa & DP da estimativa & Média dos DPs \\
\hline$\alpha$ & 1 & 1,0473 & 0,0927 & 0,0973 \\
\hline$\beta_{1}$ & -1 & $-1,0102$ & 0,2561 & 0,2456 \\
\hline$\gamma_{0}$ & -1 & $-0,9244$ & 0,3161 & 0,3357 \\
\hline$\gamma_{1}$ & 1 & 1,0093 & 0,3292 & 0,2658 \\
\hline$\sigma_{V}^{2}$ & 0,5 & 0,6279 & 0,1943 & 0,1871 \\
\hline
\end{tabular}

Note que, como no caso bayesiano, a diferença entre as médias das estimativas e os valores verdadeiros são, quase sempre, menores do que um desvio-padrão, o que indica que os resultados obtidos via simulação estão bons. 
Note ainda que os desvios-padrão das médias estão próximos aos desvios-médio em praticamente todos os casos. Isso é um bom indício de que os desvios estão sendo bem estimados pelas segundasderivadas da log-verossimilhança, ou seja, pela informação de Fisher.

As Figuras de 3.7 a 3.9 mostram os histogramas feitos a partir das estimativas calculadas nas 50 simulações, para cada um dos parâmetros.
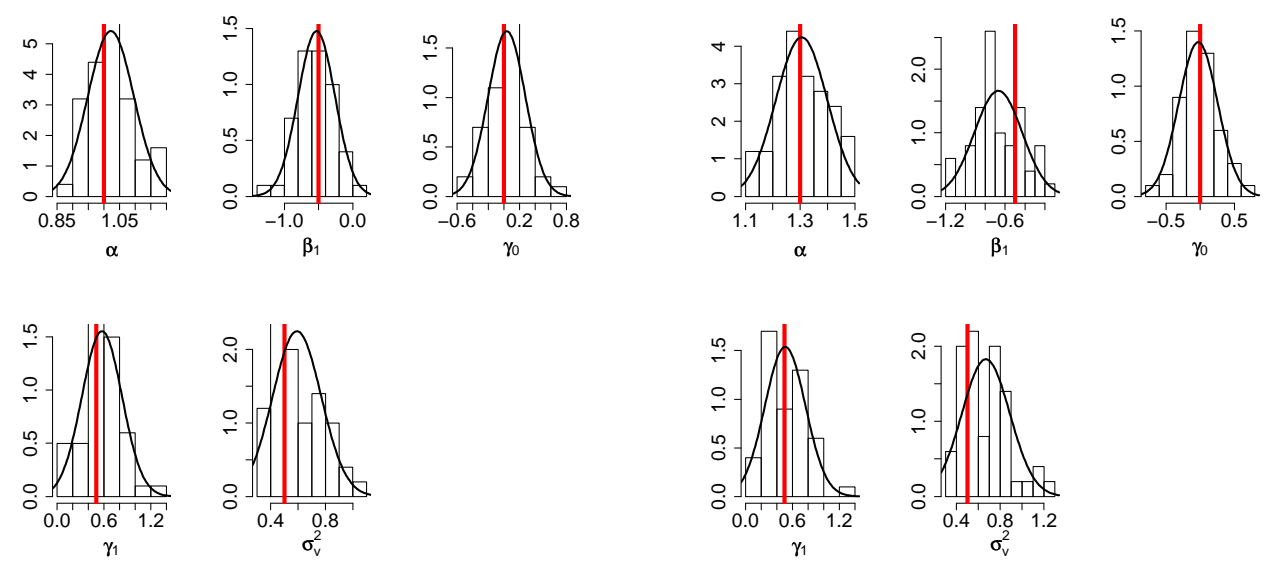

(a) Grupo 1

(b) Grupo 2
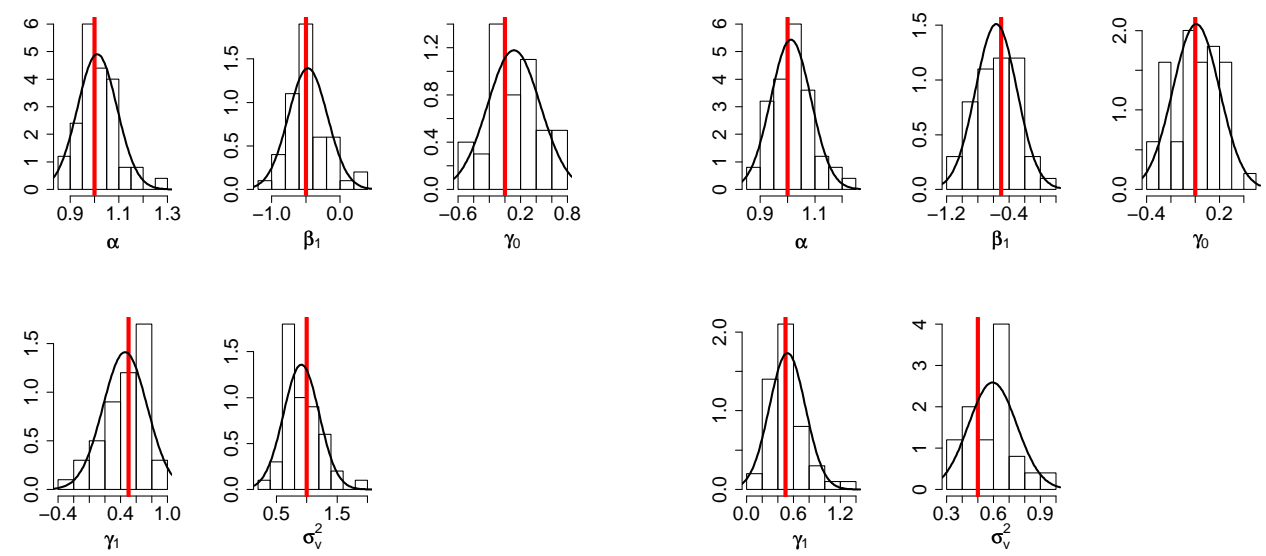

(c) Grupo 3

(d) Grupo 4

Figura 3.7: Histogramas das estimativas dos parâmetros estimados via REML $-n_{k}=15$ - parte 1 

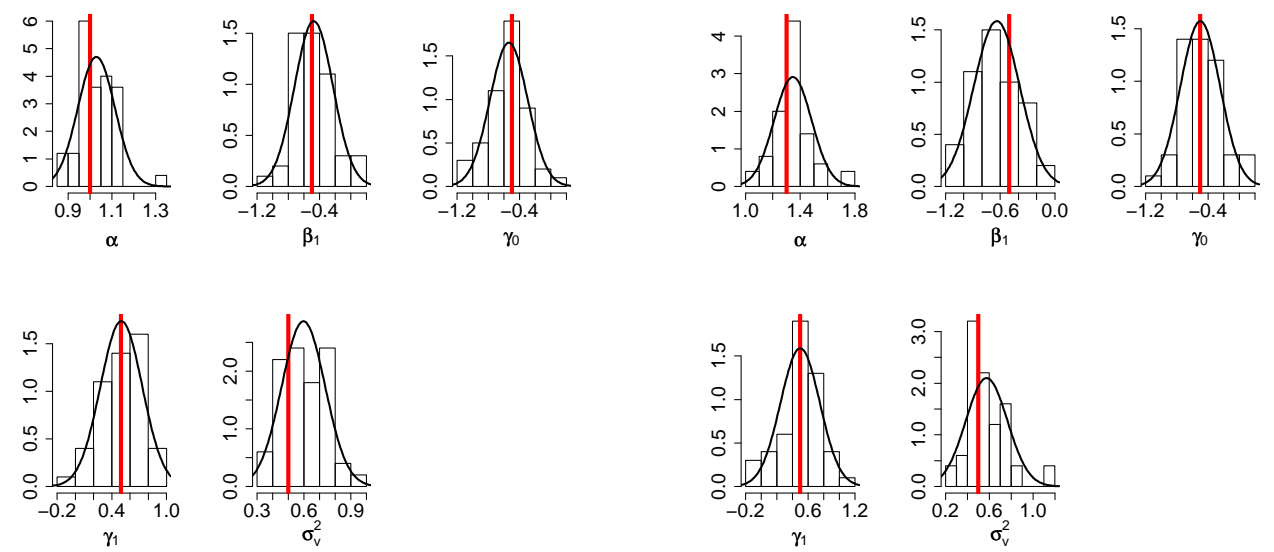

(a) Grupo 5

(b) Grupo 6
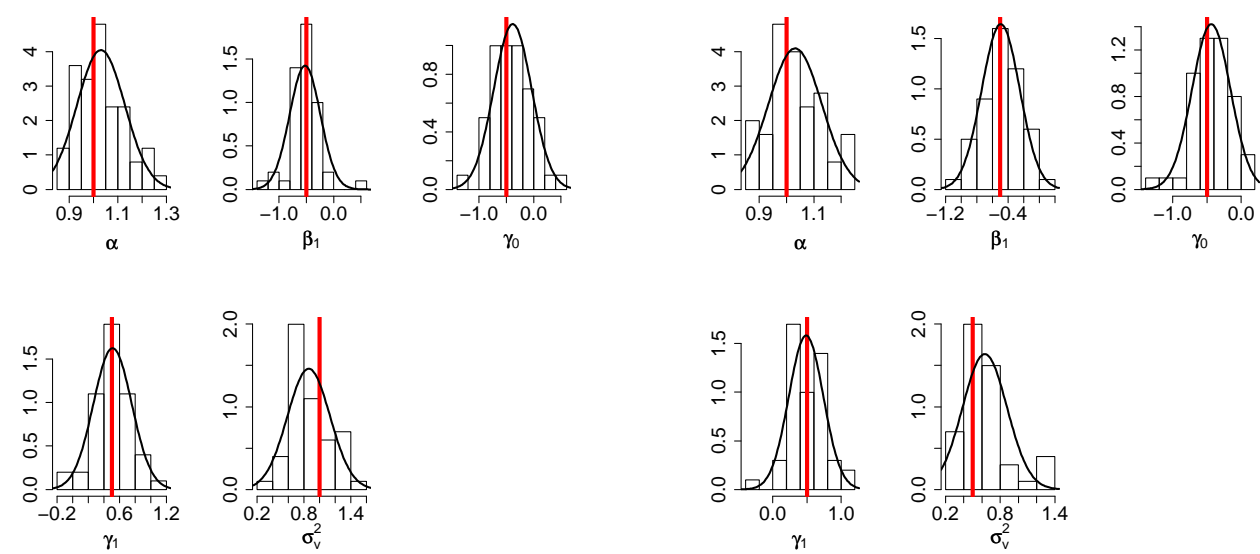

(c) Grupo 7

(d) Grupo 8

Figura 3.8: Histogramas das estimativas dos parâmetros estimados via REML $-n_{k}=15$ - parte 2 

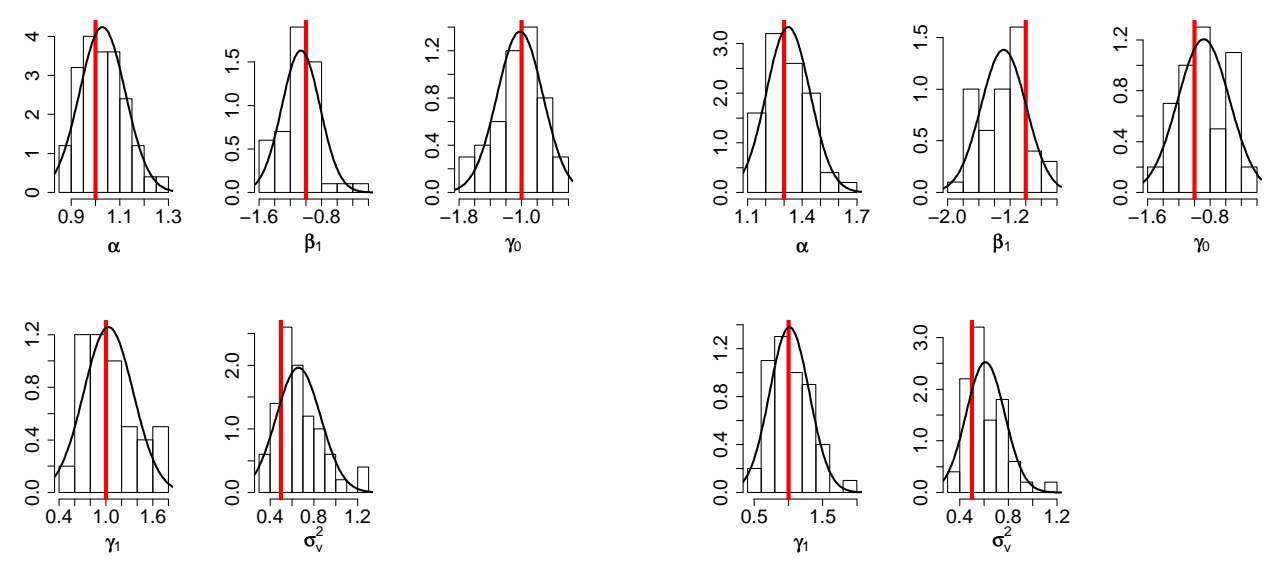

(a) Grupo 9

(b) Grupo 10
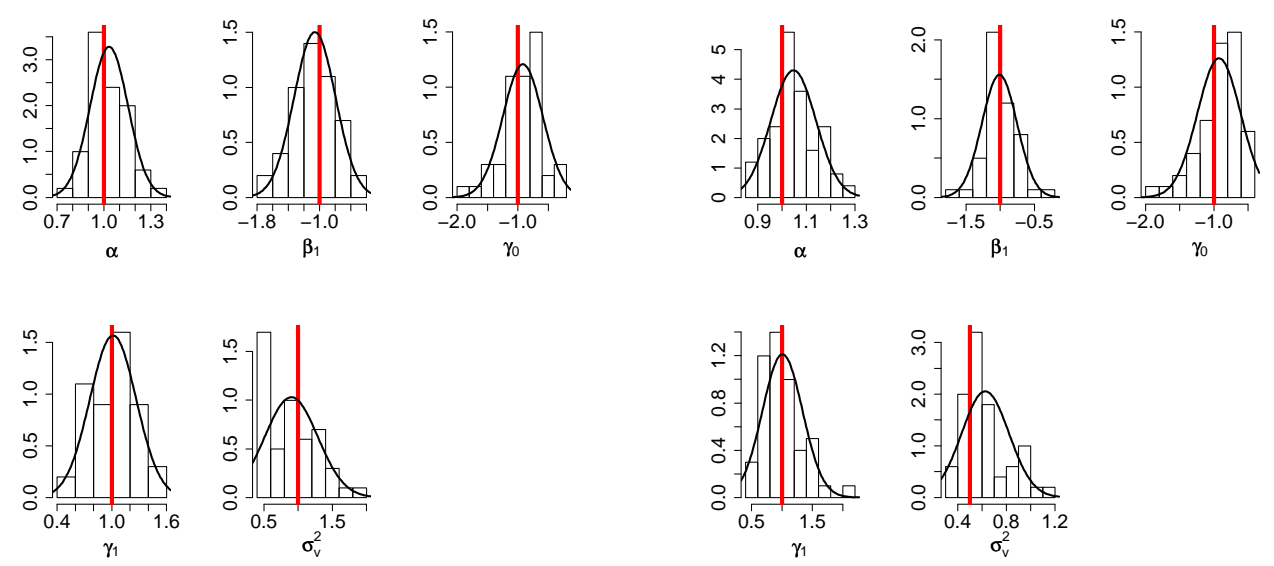

(c) Grupo 11

(d) Grupo 12

Figura 3.9: Histogramas das estimativas dos parâmetros estimados via REML $-n_{k}=15$ - parte 3

Estes histogramas indicam que as estimativas obtidas estão em torno dos valores verdadeiros, representados pelas retas verticais. Indicam também que as estimativas não estão muito longe da normalidade assintótica.

\subsubsection{Caso $n_{k}=50$}

Os resultados obtidos estão na Tabela 3.6, em que a terceira coluna apresenta a média das estimativas obtidas nas 50 simulações. Na quarta coluna está o desvio padrão calculado a partir dos 50 valores de estimativas de cada parâmetro. E, a quinta coluna contém a média dos desvios-padrão encontrados em cada uma das 50 simulações. Estes desvios-padrão foram obtidos pela raiz quadrada dos elementos da diagonal principal da matriz de informação de Fisher. 
Tabela 3.6: Resultado de 50 simulações via REML para $n_{k}=50$

\begin{tabular}{|c|c|c|c|c|}
\hline \multicolumn{5}{|c|}{ Grupo 1: $\left(\alpha=1 ; \sigma_{V}^{2}=0,5 ;\right.$ Censura $\left.=500 ; \beta_{1}=-0,5 ; \gamma_{0}=0 ; \gamma_{1}=0,5\right)$} \\
\hline Parâmetro & Valor verdadeiro & Média da estimativa & DP da estimativa & Média dos DPs \\
\hline$\alpha$ & 1 & 1,0027 & 0,0385 & 0,0405 \\
\hline$\beta_{1}$ & $-0,5$ & $-0,5195$ & 0,1106 & 0,1252 \\
\hline$\gamma_{0}$ & 0 & 0,0468 & 0,1924 & 0,2920 \\
\hline$\gamma_{1}$ & 0,5 & 0,4971 & 0,1059 & 0,1268 \\
\hline$\sigma_{V}^{2}$ & 0,5 & 0,5940 & 0,2235 & 0,1447 \\
\hline \multicolumn{5}{|c|}{ Grupo 2: $\left(\alpha=1,3 ; \sigma_{V}^{2}=0,5 ;\right.$ Censura $\left.=500 ; \beta_{1}=-0,5 ; \gamma_{0}=0 ; \gamma_{1}=0,5\right)$} \\
\hline Parâmetro & Valor verdadeiro & Média da estimativa & DP da estimativa & Média dos DPs \\
\hline$\alpha$ & 1,3 & 1,3079 & 0,0554 & 0,0536 \\
\hline$\beta_{1}$ & $-0,5$ & $-0,6408$ & 0,1311 & 0,1245 \\
\hline$\gamma_{0}$ & 0 & $-0,0138$ & 0,2375 & 0,2923 \\
\hline$\gamma_{1}$ & 0,5 & 0,5232 & 0,1266 & 0,1264 \\
\hline$\sigma_{V}^{2}$ & 0,5 & 0,5499 & 0,2178 & 0,1335 \\
\hline \multicolumn{5}{|c|}{ Grupo 3: $\left(\alpha=1 ; \sigma_{V}^{2}=1 ;\right.$ Censura $\left.=500 ; \beta_{1}=-0,5 ; \gamma_{0}=0 ; \gamma_{1}=0,5\right)$} \\
\hline Parâmetro & Valor verdadeiro & Média da estimativa & DP da estimativa & Média dos DPs \\
\hline$\alpha$ & 1 & 0,9963 & 0,0384 & 0,0410 \\
\hline$\beta_{1}$ & $-0,5$ & $-0,4956$ & 0,1486 & 0,1312 \\
\hline$\gamma_{0}$ & 0 & 0,0275 & 0,2867 & 0,2931 \\
\hline$\gamma_{1}$ & 0,5 & 0,4911 & 0,1301 & 0,1322 \\
\hline$\sigma_{V}^{2}$ & 1 & 0,9873 & 0,3615 & 0,2399 \\
\hline \multicolumn{5}{|c|}{ Grupo 4: $\left(\alpha=1 ; \sigma_{V}^{2}=0,5 ;\right.$ Censura $\left.=300 ; \beta_{1}=-0,5 ; \gamma_{0}=0 ; \gamma_{1}=0,5\right)$} \\
\hline Parâmetro & Valor verdadeiro & Média da estimativa & DP da estimativa & Média dos DPs \\
\hline$\alpha$ & 1 & 1,0094 & 0,0432 & 0,0415 \\
\hline$\beta_{1}$ & $-0,5$ & $-0,5219$ & 0,1506 & 0,1223 \\
\hline$\gamma_{0}$ & 0 & $-0,0185$ & 0,2279 & 0,2922 \\
\hline$\gamma_{1}$ & 0,5 & 0,5089 & 0,1097 & 0,1253 \\
\hline$\sigma_{V}^{2}$ & 0,5 & 0,4991 & 0,1759 & 0,1209 \\
\hline \multicolumn{5}{|c|}{ Grupo 5: $\left(\alpha=1 ; \sigma_{V}^{2}=0,5 ;\right.$ Censura $\left.=500 ; \beta_{1}=-0,5 ; \gamma_{0}=-0,5 ; \gamma_{1}=0,5\right)$} \\
\hline Parâmetro & Valor verdadeiro & Média da estimativa & DP da estimativa & Média dos DPs \\
\hline$\alpha$ & 1 & 0,9965 & 0,0446 & 0,0468 \\
\hline$\beta_{1}$ & $-0,5$ & $-0,4912$ & 0,1379 & 0,1236 \\
\hline$\gamma_{0}$ & $-0,5$ & $-0,5062$ & 0,2489 & 0,2957 \\
\hline$\gamma_{1}$ & 0,5 & 0,5172 & 0,1200 & 0,1341 \\
\hline$\sigma_{V}^{2}$ & 0,5 & 0,5851 & 0,2127 & 0,1453 \\
\hline \multicolumn{5}{|c|}{ Grupo 6: $\left(\alpha=1,3 ; \sigma_{V}^{2}=0,5 ;\right.$ Censura $\left.=500 ; \beta_{1}=-0,5 ; \gamma_{0}=-0,5 ; \gamma_{1}=0,5\right)$} \\
\hline Parâmetro & Valor verdadeiro & Média da estimativa & DP da estimativa & Média dos DPs \\
\hline$\alpha$ & 1,3 & 1,3122 & 0,0698 & 0,0614 \\
\hline$\beta_{1}$ & $-0,5$ & $-0,6343$ & 0,1059 & 0,1249 \\
\hline$\gamma_{0}$ & $-0,5$ & $-0,5215$ & 0,2583 & 0,2958 \\
\hline$\gamma_{1}$ & 0,5 & 0,5331 & 0,1436 & 0,1342 \\
\hline$\sigma_{V}^{2}$ & 0,5 & 0,5582 & 0,2069 & 0,1384 \\
\hline
\end{tabular}




\begin{tabular}{|c|c|c|c|c|}
\hline \multicolumn{5}{|c|}{ Grupo 7: $\left(\alpha=1 ; \sigma_{V}^{2}=1 ;\right.$ Censura $\left.=500 ; \beta_{1}=-0,5 ; \gamma_{0}=-0,5 ; \gamma_{1}=0,5\right)$} \\
\hline Parâmetro & Valor verdadeiro & Média da estimativa & DP da estimativa & Média dos DPs \\
\hline$\alpha$ & 1 & 0,9992 & 0,0470 & 0,0454 \\
\hline$\beta_{1}$ & $-0,5$ & $-0,4957$ & 0,1401 & 0,1290 \\
\hline$\gamma_{0}$ & $-0,5$ & $-0,3928$ & 0,3272 & 0,2959 \\
\hline$\gamma_{1}$ & 0,5 & 0,5053 & 0,1181 & 0,1357 \\
\hline$\sigma_{V}^{2}$ & 1 & 0,9287 & 0,3993 & 0,2275 \\
\hline \multicolumn{5}{|c|}{ Grupo 8: $\left(\alpha=1 ; \sigma_{V}^{2}=0,5 ;\right.$ Censura $\left.=300 ; \beta_{1}=-0,5 ; \gamma_{0}=-0,5 ; \gamma_{1}=0,5\right)$} \\
\hline Parâmetro & Valor verdadeiro & Média da estimativa & DP da estimativa & Média dos DPs \\
\hline$\alpha$ & 1 & 1,0032 & 0,0497 & 0,0467 \\
\hline$\beta_{1}$ & $-0,5$ & $-0,5298$ & 0,1347 & 0,1232 \\
\hline$\gamma_{0}$ & $-0,5$ & $-0,4773$ & 0,2540 & 0,2954 \\
\hline$\gamma_{1}$ & 0,5 & 0,5178 & 0,1187 & 0,1329 \\
\hline$\sigma_{V}^{2}$ & 0,5 & 0,5470 & 0,2131 & 0,1353 \\
\hline \multicolumn{5}{|c|}{ Grupo 9: $\left(\alpha=1 ; \sigma_{V}^{2}=0,5 ;\right.$ Censura $\left.=500 ; \beta_{1}=-1 ; \gamma_{0}=-1 ; \gamma_{1}=1\right)$} \\
\hline Parâmetro & Valor verdadeiro & Média da estimativa & DP da estimativa & Média dos DPs \\
\hline$\alpha$ & 1 & 1,0030 & 0,0582 & 0,0515 \\
\hline$\beta_{1}$ & -1 & $-1,0020$ & 0,1279 & 0,1313 \\
\hline$\gamma_{0}$ & -1 & $-1,0107$ & 0,2245 & 0,3008 \\
\hline$\gamma_{1}$ & 1 & 1,0208 & 0,1367 & 0,1439 \\
\hline$\sigma_{V}^{2}$ & 0,5 & 0,5186 & 0,1883 & 0,1304 \\
\hline \multicolumn{5}{|c|}{ Grupo 10: $\left(\alpha=1,3 ; \sigma_{V}^{2}=0,5 ;\right.$ Censura $\left.=500 ; \beta_{1}=-1 ; \gamma_{0}=-1 ; \gamma_{1}=1\right)$} \\
\hline Parâmetro & Valor verdadeiro & Média da estimativa & DP da estimativa & Média dos DPs \\
\hline$\alpha$ & 1,3 & 1,3160 & 0,0887 & 0,0685 \\
\hline$\beta_{1}$ & -1 & $-1,3137$ & 0,1803 & 0,1396 \\
\hline$\gamma_{0}$ & -1 & $-1,0474$ & 0,2483 & 0,3015 \\
\hline$\gamma_{1}$ & 1 & 1,0258 & 0,1656 & 0,1458 \\
\hline$\sigma_{V}^{2}$ & 0,5 & 0,5740 & 0,2247 & 0,1447 \\
\hline \multicolumn{5}{|c|}{ Grupo 11: $\left(\alpha=1 ; \sigma_{V}^{2}=1 ;\right.$ Censura $\left.=500 ; \beta_{1}=-1 ; \gamma_{0}=-1 ; \gamma_{1}=1\right)$} \\
\hline Parâmetro & Valor verdadeiro & Média da estimativa & DP da estimativa & Média dos DPs \\
\hline$\alpha$ & 1 & 1,0108 & 0,0497 & 0,0520 \\
\hline$\beta_{1}$ & -1 & $-0,9855$ & 0,1199 & 0,1363 \\
\hline$\gamma_{0}$ & -1 & $-1,0215$ & 0,3297 & 0,3022 \\
\hline$\gamma_{1}$ & 1 & 0,9861 & 0,1367 & 0,1478 \\
\hline$\sigma_{V}^{2}$ & 1 & 0,9252 & 0,3280 & 0,2313 \\
\hline \multicolumn{5}{|c|}{ Grupo 12: $\left(\alpha=1 ; \sigma_{V}^{2}=0,5 ;\right.$ Censura $\left.=300 ; \beta_{1}=-1 ; \gamma_{0}=-1 ; \gamma_{1}=1\right)$} \\
\hline Parâmetro & Valor verdadeiro & Média da estimativa & DP da estimativa & Média dos DPs \\
\hline$\alpha$ & 1 & 1,0109 & 0,0515 & 0,0530 \\
\hline$\beta_{1}$ & -1 & $-1,0146$ & 0,1390 & 0,1327 \\
\hline$\gamma_{0}$ & -1 & $-1,0572$ & 0,2504 & 0,3016 \\
\hline$\gamma_{1}$ & 1 & 1,0147 & 0,1505 & 0,1459 \\
\hline$\sigma_{V}^{2}$ & 0,5 & 0,5416 & 0,2029 & 0,1371 \\
\hline
\end{tabular}

Note que, a diferença entre os valores médios estimados e os valores verdadeiros são menores do que um desvio-padrão, o que indica que os resultados obtidos via simulação estão bons. Note também que esta diferença é menor do que no caso $n_{k}=15$. Ou seja, como no caso bayesiano, o fato de termos mais indivíduos em cada clínica nos fornece valores estimados mais acurados. 
Note ainda que, como no caso $n_{k}=15$, os desvios-padrão das médias estão próximos aos desviosmédio em praticamente todos os casos. Isso é um bom indício de que os desvios estão sendo bem estimados pelas segundas-derivadas da log-verossimilhança, ou seja, pela informação de Fisher. Somente para o parâmetro $\sigma_{V}^{2}$ esta diferença é um pouco maior.

As Figuras de 3.10 a 3.12 mostram os histogramas feitos a partir das estimativas calculadas nas 50 simulações, para cada um dos parâmetros.
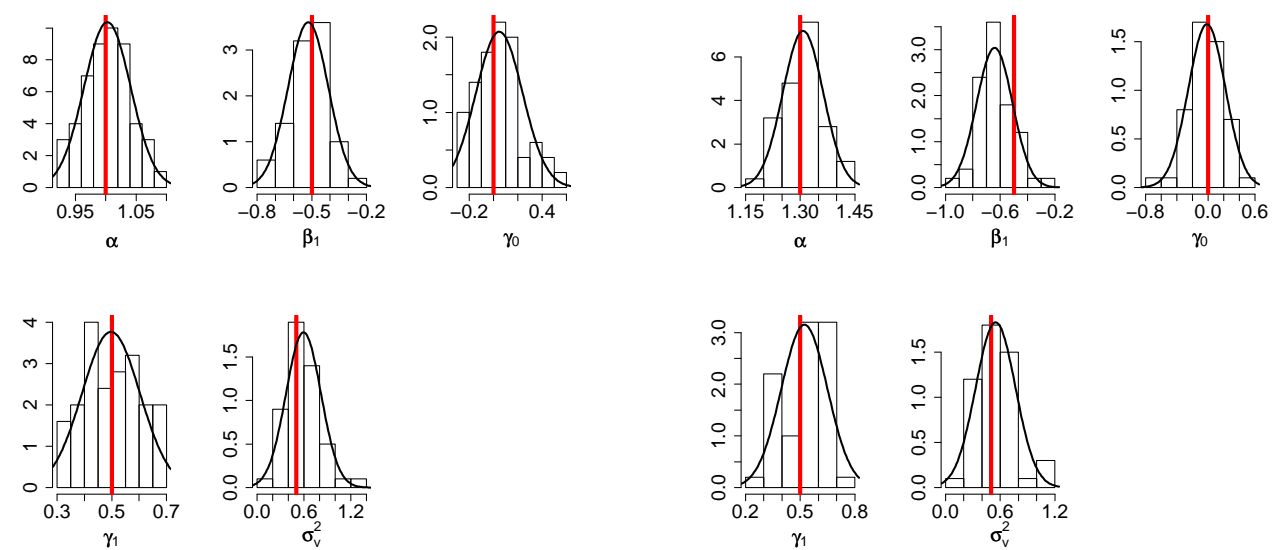

(a) Grupo 1

(b) Grupo 2
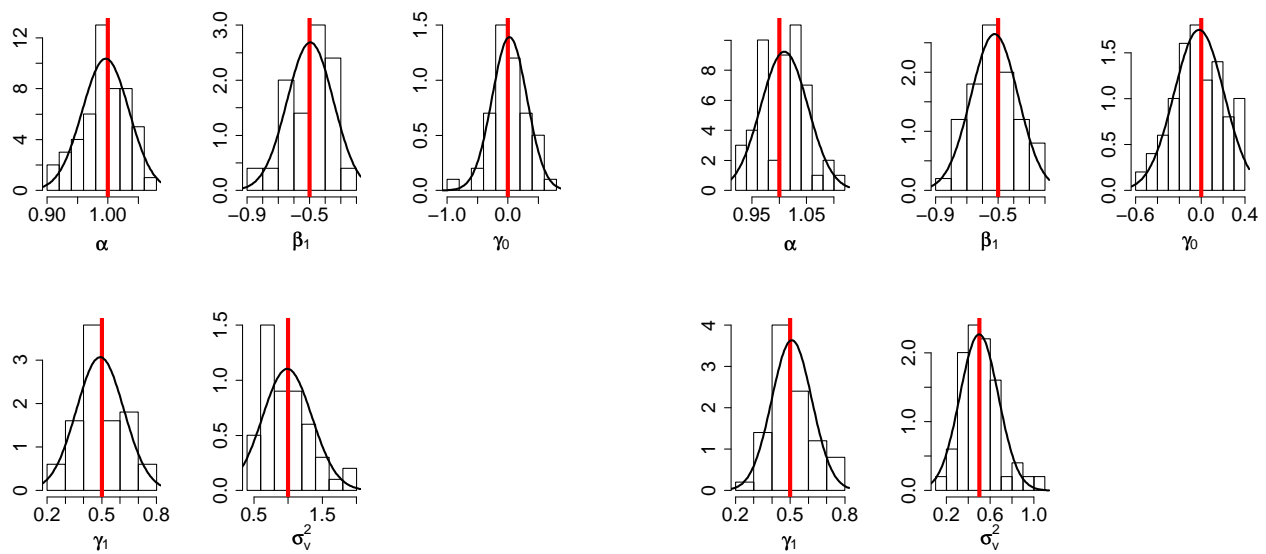

(c) Grupo 3

(d) Grupo 4

Figura 3.10: Histogramas das estimativas dos parâmetros estimados via REML $-n_{k}=50-$ parte 1 

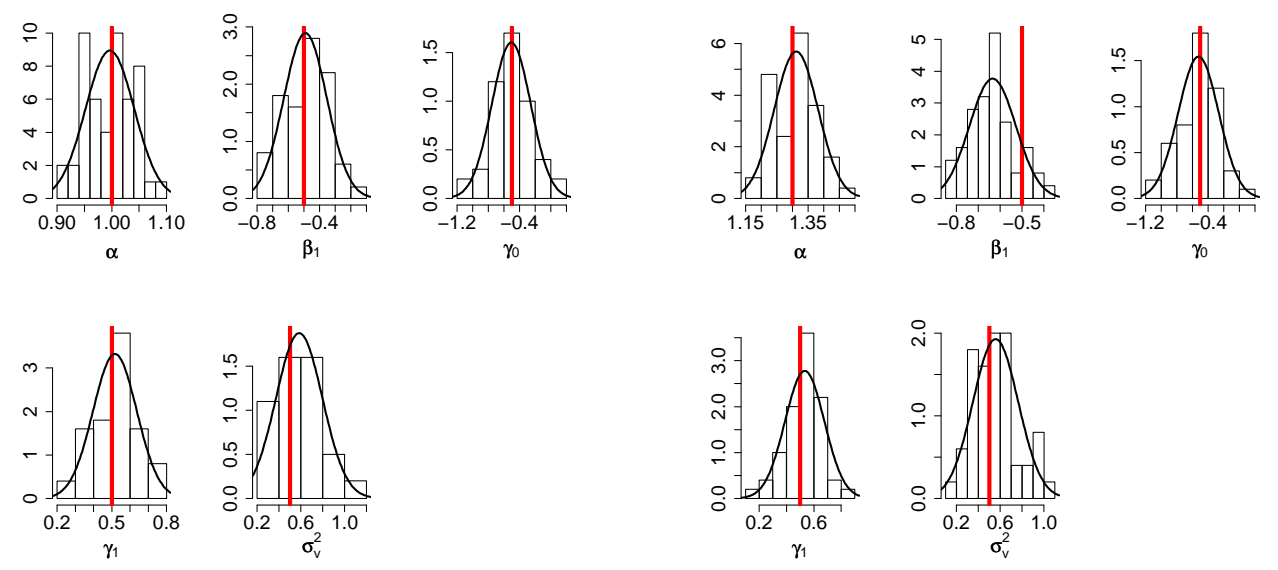

(a) Grupo 5

(b) Grupo 6
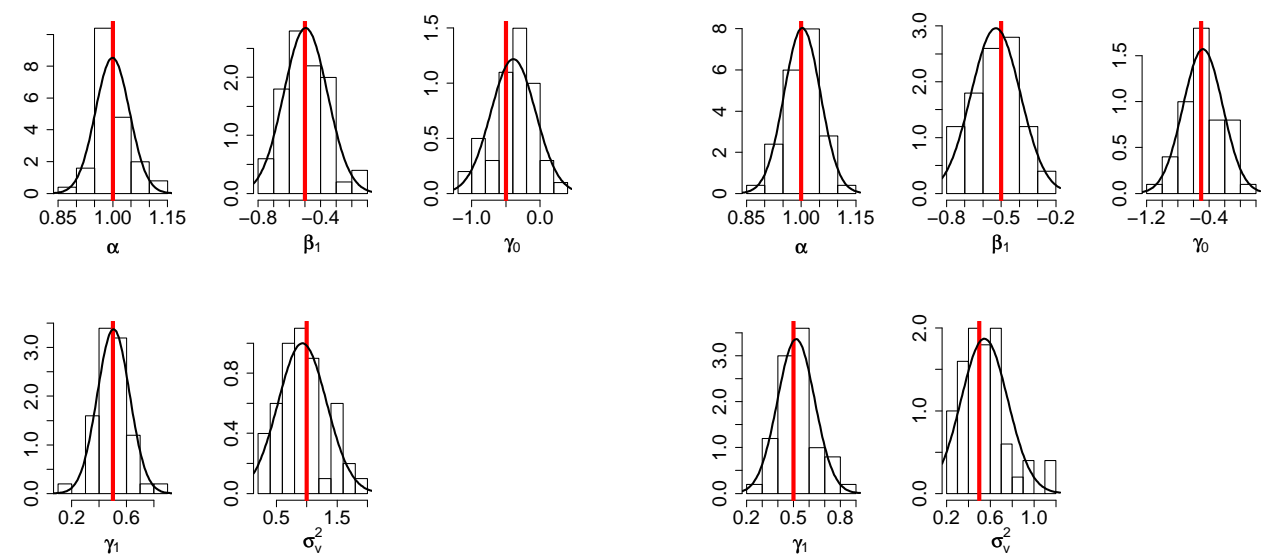

(c) Grupo 7

(d) Grupo 8

Figura 3.11: Histogramas das estimativas dos parâmetros estimados via REML $-n_{k}=50$ - parte 2 

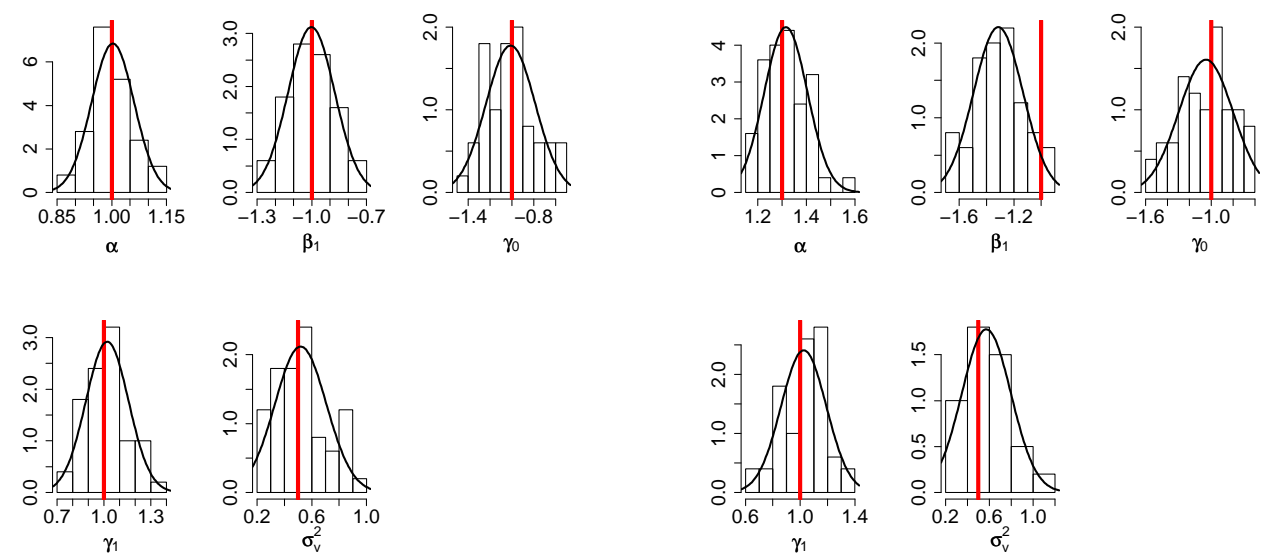

(a) Grupo 9

(b) Grupo 10
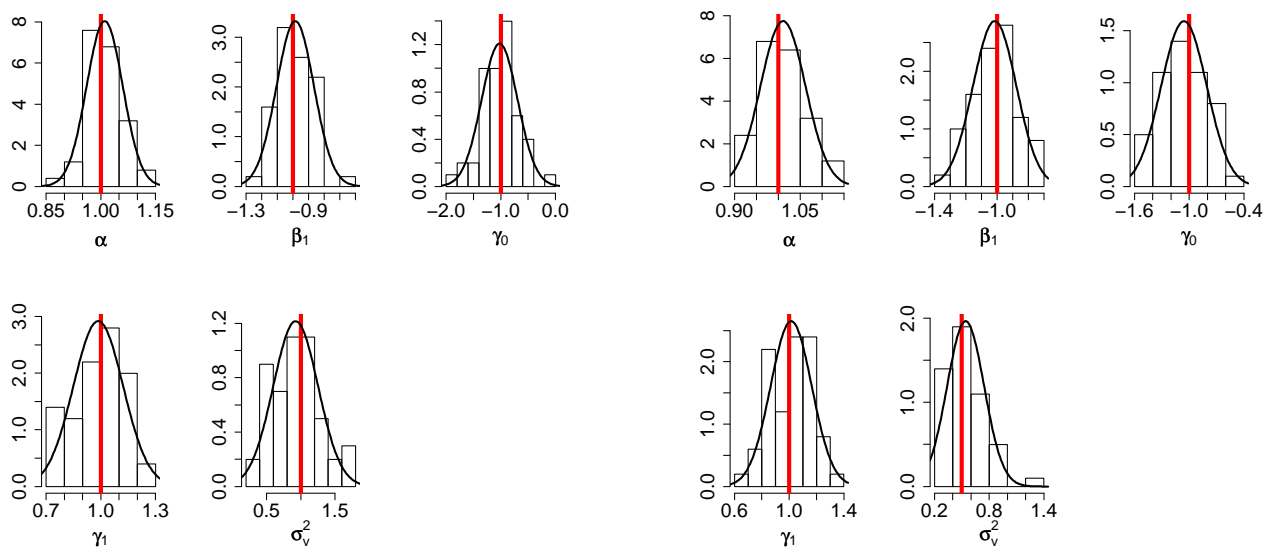

(c) Grupo 11

(d) Grupo 12

Figura 3.12: Histogramas das estimativas dos parâmetros estimados via REML $-n_{k}=50-$ parte 3

Estes histogramas indicam que as estimativas obtidas estão em torno dos valores verdadeiros, representados pelas retas verticais. Indicam, também, que as estimativas não estão muito longe da normalidade assintótica, como em todos os casos estudados.

\subsection{Conclusões}

Os resultados, tanto bayesianos quanto clássicos, mostram que os valores obtidos nas estimativas estão próximos aos valores reais, o que sugere que as estimações utilizando ambos os métodos de inferência implementados foram adequadas. Para se afirmar isso com mais segurança, o ideal seria fazer mais simulações, possivelmente para um número maior de valores de parâmetros.

Praticamente em todos os casos, a diferença entre a média do valor estimado e o valor verdadeiro é menor do que um desvio-padrão, o que sugere que os resultados estão razoavelmente precisos. 
Os histogramas feitos com os valores estimados nas simulações ilustram que os valores verdadeiros, representados pelas retas verticais, estão dentro do esperado. E, que os valores têm distribuições aproximadamente normais.

Com relação à convergência das cadeias, na abordagem bayesiana, os valores de $\sqrt{\hat{R}}$ estão bem próximos a 1 para todas as estimativas de interesse indicando que houve a convergência das cadeias e que foi atingida a distribuição de cada estimador.

Nas simulações clássica e bayesiana, os valores estimados no caso $n_{k}=50$ estão mais próximos dos valores verdadeiros do que no caso $n_{k}=15$, mas em ambos os casos os valores estimados e verdadeiros diferem, quase sempre, por uma quantidade menor que um desvio-padrão.

Note, também, que, em ambas as abordagens, a diferença entre a média das estimativas e o valor verdadeiro para o caso $n_{k}=50$ é menor do que no caso $n_{k}=15$. Ou seja, o fato de termos mais indivíduos em cada clínica nos fornece valores estimados mais acurados.

Com relação aos desvios-padrão das estimativas, é perceptível que os valores estão, quase sempre, menores no caso $n_{k}=50$ em comparação com $n_{k}=15$. A exceção ocorre no caso de $\sigma_{v}^{2}$ em que essa situação se inverte, mas a acurácia do valor estimado é melhor em $n_{k}=50$ do que em $n_{k}=15$. Isso em ambas as inferências bayesiana e clássica. 
44CAPÍTULO 3. ESTUDOS DE SIMULAÇÃO - MODELO DE CHEN-IBRAHIM-SINHA COM EFEITOS A 


\section{Capítulo 4}

\section{Análise de dados - modelo de Chen-Ibrahim-Sinha com efeitos aleatórios}

Para ilustrar uma aplicação de uso do modelo de mistura com efeitos aleatórios, Yau e Ng (2001) utilizaram um conjunto de dados em Kalbfleisch e Prentice (1980, pp.225-229). Tais dados são parte de um estudo feito pelo Radiation Therapy Oncology Group nos Estados Unidos. A seguir, é apresentada a utilização deste mesmo conjunto de dados no modelo apresentado no Capítulo 2, para fração de cura com efeitos aleatórios, a partir do modelo de Chen-Ibrahim-Sinha.

\subsection{Dados utilizados}

Os dados são de pacientes, de seis clínicas, com carcinomas na orofaringe. Ao entrarem no estudo, os pacientes eram aleatoriamente encaminhados para um dos dois grupos de tratamentos: radioterapia somente ou radioterapia associada a um agente quimioterápico. Foram coletados dados de muitas covariáveis que poderiam estar relacionadas com a sobrevivência do paciente, dentre elas estão sexo, estágio do tumor ("estadio"), estágio de nódulos linfáticos, idade, grau de diferenciação do tumor, e condições funcionais do paciente na hora do diagnóstico.

O estudo visava identificar fatores de risco, determinar a extensão do efeito dos fatores de risco e comparar os dois tipos de tratamentos em relação à sobrevivência do paciente. Os tratamentos foram aplicados nos pacientes por um período de 90 dias, após os quais cada paciente recebeu cuidados médicos pelas instituições participantes do estudo. Neste período, após os 90 dias, a única restrição para as clínicas era o tipo de tratamento em estudo. Por não haver mais nenhuma limitação quanto à continuação no tratamento, as diferentes clínicas puderam apresentar diversos tratamentos e facilidades. Isto indica que possa ter havido uma diferença de resultados (efeitos) para as diferentes clínicas, sendo importante que o modelo a estudar os resultados considere o efeito da clínica no tempo de sobrevivência dos pacientes e no número de indivíduos que se curam, levando-se em consideração que há uma correlação entre os pacientes que estavam na mesma clínica.

Em Kalbfleisch e Prentice (1980) os efeitos de clínicas foram tratados como efeitos fixos. Em Yau e Ng (2001), os efeitos foram tratados com efeitos aleatórios, no modelo de mistura. 
Como em Yau e Ng (2001), para a ilustração do uso do modelo apresentado no Capítulo 2, serão considerados somente os casos de carcinoma na parte faríngea da língua e o estágio do tumor para todos os pacientes, curados ou não.

O estágio do tumor mede a extensão do tumor em que

- $T_{1}$ se refere a tumores primários, em que o maior diâmetro é menor ou igual a $2 \mathrm{~cm}$;

- $T_{2}$ se refere a tumores primários, em que o maior diâmetro mede de $2 \mathrm{~cm}$ a $4 \mathrm{~cm}$;

- $T_{3}$ se refere a tumores primários, em que o maior diâmetro é maior ou igual a $4 \mathrm{~cm}$;

- $T_{4}$ se refere a tumores maciços espalhados para tecidos adjacentes.

A partir dos dados de estágio do tumor, é considerada a função indicadora de estágio do tumor, dada por

$$
X= \begin{cases}0, & \text { se } T_{1}, T_{2}, \text { ou } T_{3} \\ 1, & \text { se } T_{4}\end{cases}
$$

O evento "falha"é considerado morte pelo carcinoma específico. Mortes por outras causas são tratadas como censuras. Por esta definição, alguns pacientes podem nunca apresentar o evento, principalmente aqueles que respondem de modo satisfatório ao tratamento. Estes pacientes podem ser considerados curados, sendo adequado o uso de um modelo que permita a inclusão de uma fração de curados. É razoável pensar que a fração de cura depende do estágio do tumor, sendo este fator levado em consideração na modelagem do problema.

Na Tabela 4.1 são apresentados os dados a serem utilizados, em que Tempo é o número de dias que o paciente sobreviveu desde a data do diagnóstico, $X$ é a função definida em (4.1) e $\nu$ é a função indicadora de falha, ou seja

$$
\nu= \begin{cases}0, & \text { se censura } \\ 1, & \text { se falha }\end{cases}
$$


Tabela 4.1: Dados de Tratamento de Carcinoma na Orofaringe

\begin{tabular}{|c|c|c|c|c|c|c|c|}
\hline Clínica & Tempo & $X$ & $\nu$ & Clínica & Tempo & $X$ & $\nu$ \\
\hline 1 & 1312 & 0 & 0 & 3 & 1307 & 0 & 0 \\
\hline 1 & 1219 & 0 & 0 & 3 & 800 & 0 & 1 \\
\hline 1 & 264 & 1 & 1 & 3 & 666 & 0 & 1 \\
\hline 1 & 11 & 1 & 1 & 3 & 1060 & 0 & 0 \\
\hline 1 & 170 & 0 & 1 & 3 & 465 & 0 & 1 \\
\hline 1 & 372 & 0 & 1 & 3 & 518 & 0 & 1 \\
\hline 1 & 182 & 0 & 0 & 4 & 235 & 0 & 1 \\
\hline 1 & 723 & 1 & 0 & 4 & 324 & 0 & 1 \\
\hline 1 & 90 & 0 & 0 & 4 & 1565 & 0 & 0 \\
\hline 2 & 270 & 0 & 1 & 4 & 1234 & 0 & 0 \\
\hline 2 & 162 & 0 & 1 & 4 & 293 & 0 & 1 \\
\hline 2 & 228 & 1 & 1 & 4 & 593 & 1 & 0 \\
\hline 2 & 370 & 1 & 1 & 4 & 213 & 0 & 1 \\
\hline 2 & 805 & 1 & 1 & 5 & 245 & 1 & 1 \\
\hline 2 & 517 & 0 & 1 & 5 & 262 & 0 & 1 \\
\hline 2 & 599 & 0 & 1 & 5 & 915 & 0 & 1 \\
\hline 2 & 89 & 1 & 1 & 5 & 1377 & 1 & 0 \\
\hline 2 & 99 & 0 & 1 & 5 & 172 & 1 & 1 \\
\hline 2 & 219 & 1 & 1 & 5 & 15 & 1 & 1 \\
\hline 2 & 154 & 1 & 1 & 5 & 382 & 1 & 1 \\
\hline 2 & 266 & 1 & 1 & 5 & 363 & 0 & 1 \\
\hline 2 & 407 & 0 & 1 & 5 & 107 & 1 & 1 \\
\hline 2 & 751 & 0 & 0 & 5 & 757 & 1 & 1 \\
\hline 3 & 184 & 0 & 1 & 5 & 38 & 0 & 1 \\
\hline 3 & 1064 & 0 & 1 & 6 & 222 & 0 & 1 \\
\hline 3 & 1472 & 0 & 0 & 6 & 1644 & 0 & 0 \\
\hline 3 & 526 & 0 & 1 & 6 & 785 & 0 & 1 \\
\hline 3 & 1495 & 0 & 0 & 6 & 11 & 1 & 1 \\
\hline 3 & 661 & 0 & 1 & 6 & 1095 & 0 & 0 \\
\hline 3 & 407 & 0 & 1 & 6 & 914 & 0 & 0 \\
\hline 3 & 548 & 0 & 1 & 6 & 346 & 0 & 1 \\
\hline 3 & 1317 & 0 & 0 & 6 & 608 & 0 & 1 \\
\hline 3 & 1317 & 0 & 0 & 6 & 112 & 0 & 1 \\
\hline
\end{tabular}

Fonte: Adaptado de Kalbfleisch e Prentice (1980)

A partir dos dados da Tabela 4.1, foram feitos os gráficos de Kaplan-Meier para cada uma das seis clínicas. O fato de as curvas estabilizarem em alturas diferentes de zero pode ser um indício de que haja uma parcela de pacientes curados. Outro indício de que é adequado um estudo com efeitos de clínica é o fato de que os gráficos de Kaplan-Meier (Figura 4.1) para cada clínica estabilizarem em alturas distintas. 

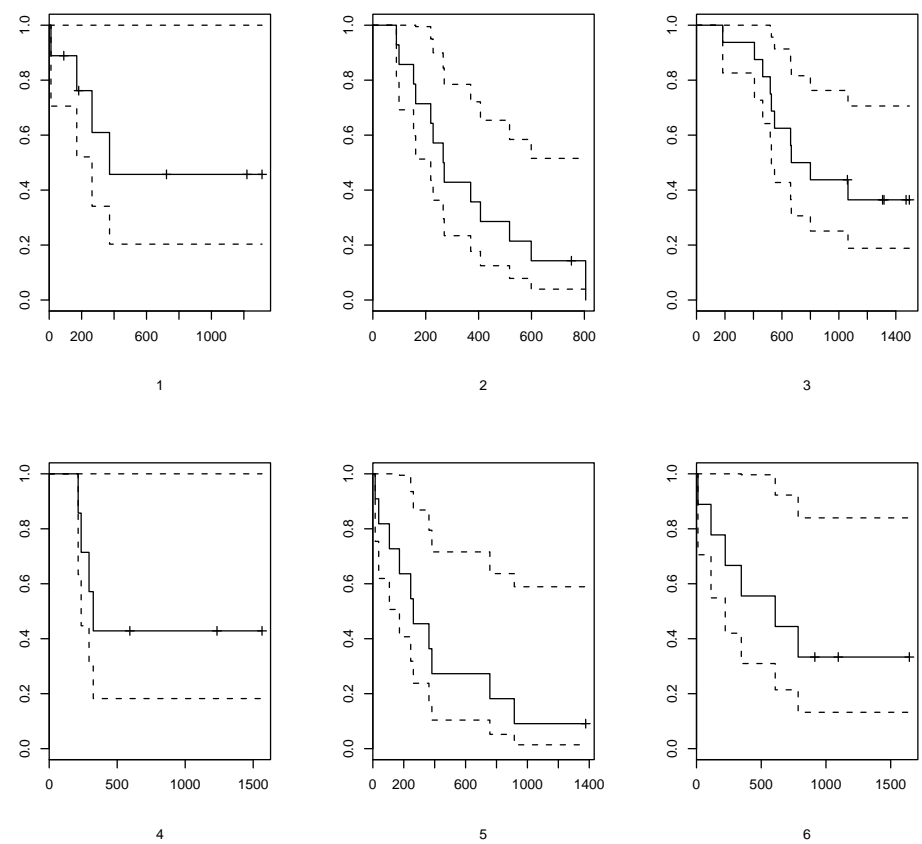

Figura 4.1: Gráficos de Kaplan-Meier para cada uma das seis clínicas

Para este conjunto de dados, o modelo apresentado no Capítulo 2 assume a forma dada em (2.2) em que

- $y_{j k} \sim \operatorname{Weibull}\left(\alpha, \lambda_{j k}\right)$;

- $\boldsymbol{\beta}=\beta_{1}$, portanto, $\lambda_{j k}=\mathbf{x}_{j k}^{\top} \boldsymbol{\beta}=\mathbf{x}_{j k} \beta_{1}$;

- $\gamma=\left[\gamma_{0}, \gamma_{1}\right]^{\top}$

- os valores de $\mathbf{x}_{j k}$ são lidos a partir da coluna $X$ da Tabela 4.1, em que $M=6$, (ou seja, $k=1,2, \ldots, 6), j=1,2, \ldots, n_{k}$ e $n_{1}=9, n_{2}=14, n_{3}=16, n_{4}=7, n_{5}=11$ e $n_{6}=9$.

\subsection{Inferência bayesiana via Metropolis-Hastings}

Uma primeira análise desses dados foi feita com a utilização do programa Winbugs (versão 1.4). Foi adotado $\alpha=1$ fixado, ou seja, $y_{j k} \sim \operatorname{Weibull}\left(\alpha, \lambda_{j k}\right)$ ou, equivalentemente, $y_{j k} \sim$ Exponencial $\left(\lambda_{j k}\right)$. Os comandos utilizados encontram-se no apêndice A.2. Os resultados obtidos estão apresentados na Tabela 4.2, em que a coluna de média apresenta o valor da média entre os valores amostrados, DP apresenta o desvio-padrão e erro MC está mostrando os valores obtidos pelo erro padrão de Monte Carlo para a média. 
Tabela 4.2: Resultados - câncer na orofaringe - modelo de Chen-Ibrahim-Sinha com efeitos aleatórios - Metropolis-Hastings

\begin{tabular}{ccccccccc}
\hline \multicolumn{7}{c}{ Resultados das posterioris, baseados em 30000 amostras } \\
\hline parâmetro & média & DP & erro MC & $\mathbf{2 , 5 0 \%}$ & mediana & $\mathbf{9 7 , 5 0 \%}$ & início & amostras \\
\hline$\beta_{1}$ & $-6,8400$ & 0,7873 & 0,0358 & $-8,7420$ & $-6,6950$ & $-5,7040$ & 4001 & 26000 \\
$\gamma_{0}$ & $-0,4617$ & 0,2365 & 0,0063 & $-0,9453$ & $-0,4572$ & $-0,0242$ & 4001 & 26000 \\
$\gamma_{1}$ & 1,4960 & 0,6874 & 0,0313 & 0,4221 & 1,3900 & 3,1560 & 4001 & 26000 \\
$\sigma_{V}^{2}$ & 0,2559 & 0,1344 & 0,00162 & 0,1022 & 0,2241 & 0,5981 & 4001 & 26000 \\
\hline
\end{tabular}

Note que $\sigma_{V}^{2}$ é muito pequeno o que indicaria que a componente de variância não é significativamente diferente de zero, de modo que não há efeito de clínica na parte da população de curados. Este resultado já tinha sido apontado em Yau e Ng (2001), em que modelaram os dados via modelo de mistura com efeitos aleatórios tanto na parcela da população que se cura quanto na que não se cura. Eles se utilizaram de uma abordagem clássica, em que os estimadores foram obtidos via máxima verossimilhança e via REML.

A Figura 4.2 mostra os histogramas feitos com os dados amostrados pelo programa Winbugs.
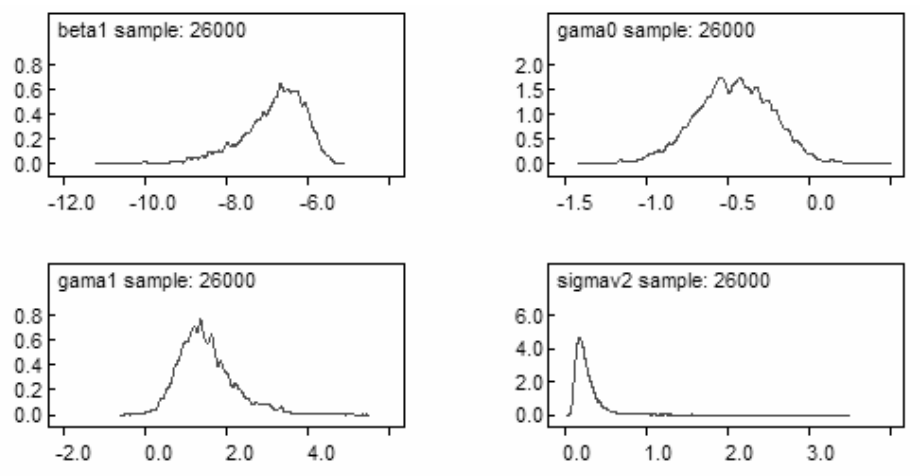

Figura 4.2: Histogramas - câncer na orofaringe - modelo de Chen-Ibrahim-Sinha com efeitos aleatórios Metropolis-Hastings

O software Winbugs, neste exemplo, executou o algoritmo Metropolis-Hastings.

Para se incluir o caso em que $\alpha \neq 1$, ou seja, a distribuição é uma Weibull não exponencial, foram feitas alterações no programa para o Winbugs, que foi então recompilado. Os novos resultados estão apresentados na Tabela 4.3. 
Tabela 4.3: Resultados - câncer na orofaringe - modelo de Chen-Ibrahim-Sinha com efeitos aleatórios - Metropolis-Hastings - com alfa incluído

\begin{tabular}{ccccccccc}
\hline \multicolumn{7}{c}{ Resultados das posterioris, baseados em 30000 amostras } \\
\hline parâmetro & média & DP & erro MC & $\mathbf{2 , 5 0 \%}$ & mediana & $\mathbf{9 7 , 5 0 \%}$ & início & amostras \\
\hline$\alpha$ & 0,1501 & 0,0151 & 0,0001 & 0,1207 & 0,1498 & 0,1798 & 4001 & 26000 \\
$\beta_{1}$ & $-3,8410$ & 1,1330 & 0,0709 & $-6,4730$ & $-3,7400$ & $-1,9800$ & 4001 & 26000 \\
$\gamma_{0}$ & $-0,3496$ & 0,2358 & 0,0059 & $-0,8307$ & $-0,3405$ & 0,0938 & 4001 & 26000 \\
$\gamma_{1}$ & 3,1090 & 1,1330 & 0,0715 & 1,2210 & 3,0120 & 5,7090 & 4001 & 26000 \\
$\sigma_{V}^{2}$ & 0,2529 & 0,1321 & 0,0015 & 0,1031 & 0,2214 & 0,5949 & 4001 & 26000 \\
\hline
\end{tabular}

Novamente, o valor estimado para $\sigma_{V}^{2}$ é muito pequeno, indicando que pode não haver diferença de efeitos entre as clínicas, para os curados.

A Figura 4.3 mostra os histogramas feitos com os dados gerados pelo programa Winbugs, neste caso em que $\alpha$ não está fixado em 1.
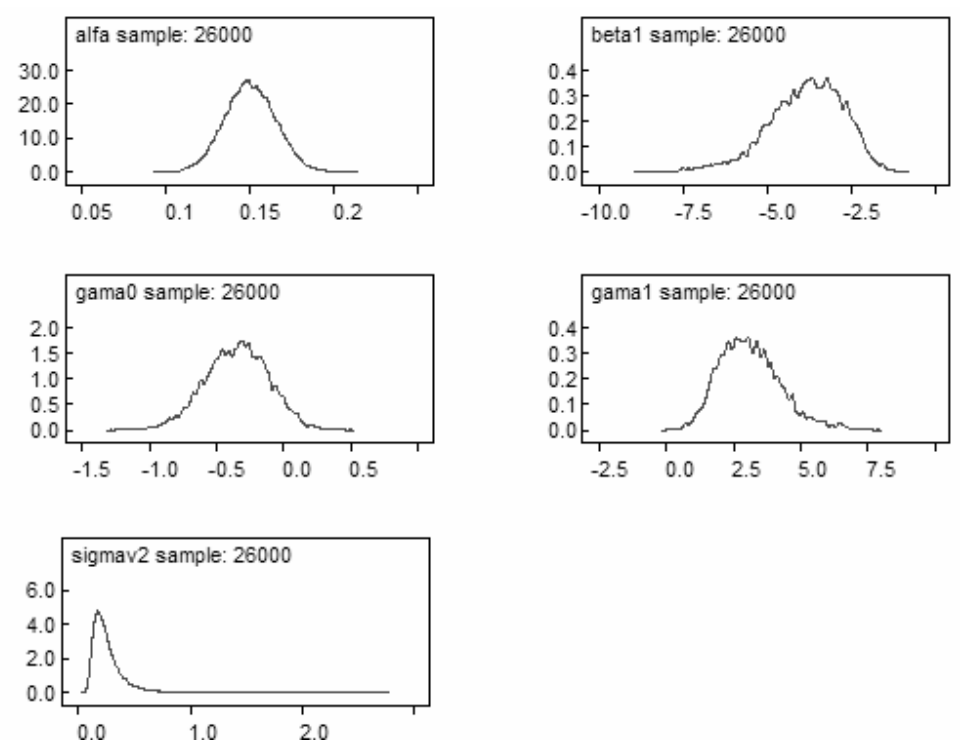

Figura 4.3: Histogramas - câncer na orofaringe - modelo de Chen-Ibrahim-Sinha com efeitos aleatórios Metropolis-Hastings - com alfa incluído

A Figura 4.4 mostra os gráficos dos valores de $\sqrt{\hat{R}}$ (Gelman e Rubin, 1992), como definidos na seção 2.2 . 

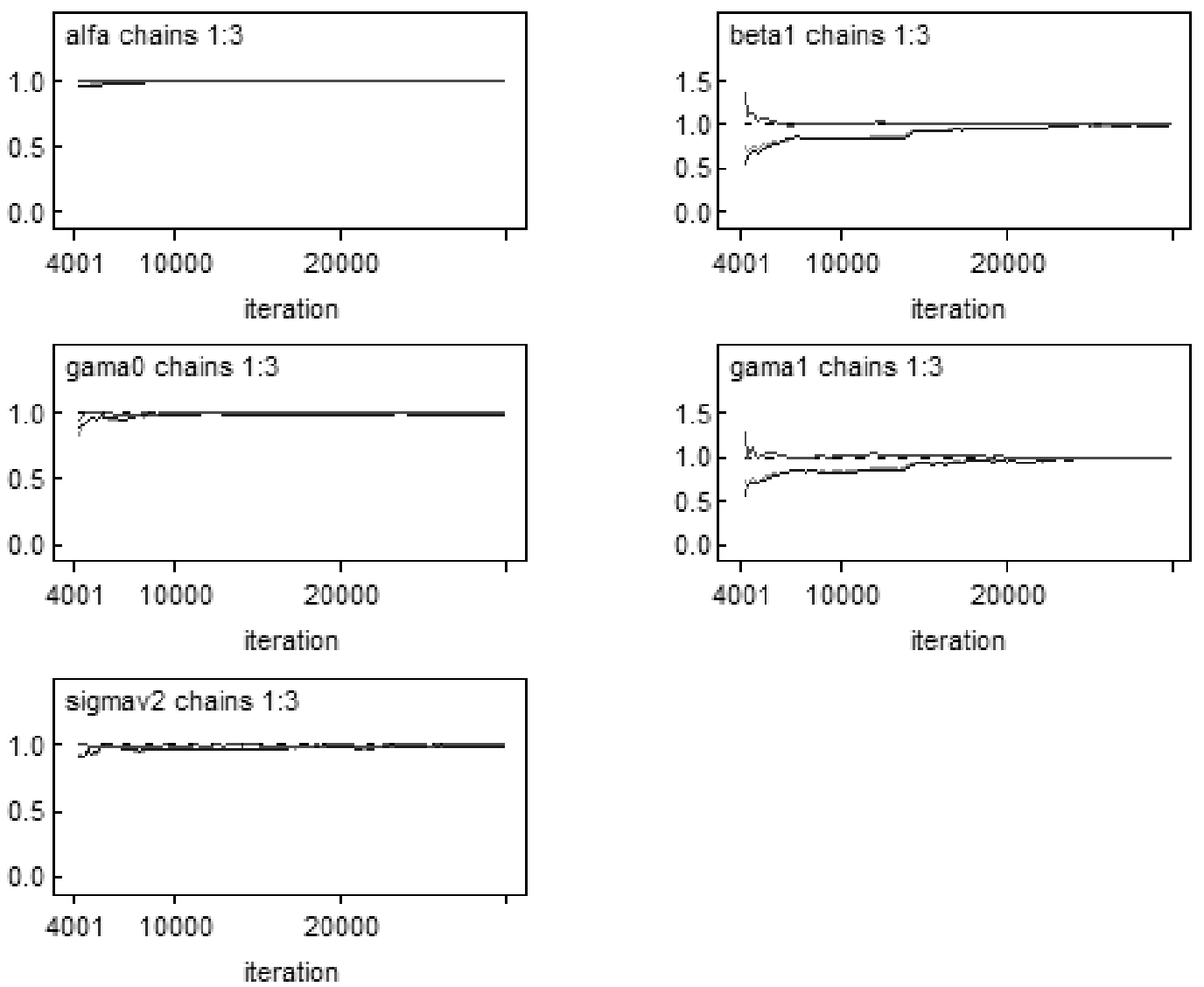

Figura 4.4: $\sqrt{\hat{R}}$ - câncer na orofaringe - modelo de Chen-Ibrahim-Sinha com efeitos aleatórios - MetropolisHastings

Note que para todos os parâmetros, os valores estão tendendo ao valor 1, o que indica que as cadeias estão convergindo e que foi atingida a distribuição de cada estimador.

\subsection{Análise clássica}

O conjunto de dados, apresentado na seção 4.1, foi novamente utilizado sendo feita a abordagem relatada na seção 2.3. Desta vez, o software utilizado para a compilação dos dados foi Maple versão 10. Os comandos estão apresentados no apêndice A.3.

Do mesmo modo que foi feito anteriormente, foram analisadas duas situações: $\alpha=1$ fixado (distribuição exponencial) e $\alpha$ não fixado, a ser estimado pelo modelo.

No primeiro caso ( $\alpha=1$ fixado), os resultados obtidos estão na Tabela 4.4. 
Tabela 4.4: Resultados - câncer na orofaringe - modelo de Chen-Ibrahim-Sinha com efeitos aleatórios - REML, com alfa fixado 1

\begin{tabular}{ccc}
\hline parâmetros & estimativa & desvio-padrão \\
\hline$\alpha$ (fixado) & 1 & - \\
$\beta_{1}$ & $-6,8388$ & 0,9432 \\
$\gamma_{0}$ & $-0,4273$ & 0,3466 \\
$\gamma_{1}$ & 1,4321 & 0,7809 \\
$\sigma_{V}^{2}$ (BLUP) & 0,0353 & - \\
$\sigma_{V}^{2}$ (REML) & 0,2187 & 0,0931 \\
\hline \multicolumn{4}{|}{} \\
\hline efeito de clínica & preditores (Blup) \\
\hline$V_{1}$ & $-0,2834$ \\
$V_{2}$ & 0,2696 \\
$V_{3}$ & $-0,0346$ \\
$V_{4}$ & $-0,1775$ \\
$V_{5}$ & 0,1270 \\
$V_{6}$ & 0,0988 \\
\end{tabular}

Note que os resultados da Tabela 4.4 estão bem próximos aos da Tabela 4.2.

Para o segundo caso, em que $\alpha$ é estimado, os resultados obtidos estão apresentados na Tabela 4.5 .

Tabela 4.5: Resultados - câncer na orofaringe - modelo de Chen-Ibrahim-Sinha com efeitos aleatórios - REML

\begin{tabular}{ccc}
\hline parâmetros & estimativa & desvio-padrão \\
\hline$\alpha$ & 0,1519 & 0,0153 \\
$\beta_{1}$ & $-11,3699$ & 52,8053 \\
$\gamma_{0}$ & $-0,3280$ & 0,3466 \\
$\gamma_{1}$ & 10,6126 & 52,8050 \\
$\sigma_{V}^{2}$ (BLUP) & 0,0311 & - \\
$\sigma_{V}^{2}$ (REML) & 0,2129 & 0,0884 \\
\hline \multicolumn{3}{c}{} \\
\hline efeito de clínica & preditores (Blup) \\
\hline$V_{1}$ & $-0,2680$ & \\
$V_{2}$ & $-0,0589$ & \\
$V_{3}$ & $-0,0589$ & \\
$V_{4}$ & $-0,1281$ & \\
$V_{5}$ & 0,1875 & \\
$V_{6}$ & 0,0245 & \\
\hline
\end{tabular}

Como no caso bayesiano, os valores estimados para $\sigma_{V}^{2}$ estão próximos de zero, indicando que pode não haver diferença entre as clínicas, para os indivíduos curados. 
Note que, comparando-se os valores nas Tabelas 4.5 e 4.3, somente os valores estimados para $\alpha$, $\gamma_{0}$ e $\sigma_{V}^{2}$ estão parecidos. Os valores estimados para $\beta_{1}$ e $\gamma_{1}$ estão muito diferentes.

Note, também, que os desvios-padrão para $\beta_{1}$ e $\gamma_{1}$, na Tabela 4.5, estão muita elevadas, o que indica que a inferência não está boa. Um motivo para a obtenção destas variâncias é o fato de os dados serem muito heterogêneos entre as clínicas: o número de pacientes nas diferentes clínicas varia de 7 a 16; a clínica 2 possui somente 1 dos 14 pacientes censurado; a clínica 3 apresenta todos os 16 pacientes com tumor primário; os tempos de falha variam de 11 a 1644, sendo o valor médio aproximadamente 575 dias.

\subsection{Conclusões}

Os resultados obtidos com a estimação bayesiana, via Metropolis-Hastings estão próximos aos obtidos com a abordagem clássica, via REML.

É nítida uma diferença de estimativas para os parâmetros $\beta_{1}$ e $\gamma_{1}$, quando estimados pelos dois métodos apresentados. Mas, para ambos, as variâncias estimadas classicamente estão muito elevadas, fato que pode ter ocorrido devido às características dos dados utilizados, como, por exemplo, baixo número de falhas em algumas clínicas, poucas observações com estágio avançado do tumor. 
54CAPÍTULO 4. ANÁLISE DE DADOS - MODELO DE CHEN-IBRAHIM-SINHA COM EFEITOS ALEATC 


\section{Capítulo 5}

\section{Modelo de mistura com efeitos aleatórios}

Neste capítulo é apresentado o modelo de mistura com efeitos aleatórios na fração de curados, que visam avaliar o efeito que determinada clínica tem, por exemplo, no tratamento de câncer de certa população que tem presença de imunes. É feita a caracterização do modelo e apresentada uma abordagem bayesiana e uma clássica para a obtenção de estimadores.

\subsection{Definindo o modelo}

Considere o modelo de mistura apresentado na seção 1.1. Suponha que foram estudados casos em $M$ clínicas e que para a $k$-ésima clínica havia $n_{k}$ indivíduos, $k=1,2, \ldots, M$. Para $j=1,2, \ldots, n_{k}$ e $k=1,2, \ldots, M$, considere $T_{j k}$ o tempo observado de falha para o indivíduo $j$ da clínica $k$. Considere ainda $N=\sum_{k=1}^{M} n_{k}$ o número total de observações. Seja $\mathbf{x}_{j k}$ o vetor de covariáveis correspondentes a $T_{j k}$. Assim, a proporção $p$ (curados), pode ser modelada por

$$
p_{j k}=1-\pi\left(\mathbf{x}_{j k}\right)=1-\frac{1}{1+e^{\mathbf{w}_{j k}^{\top} \boldsymbol{\gamma}+V_{k}}}
$$

em que $\mathbf{w}_{i}=\left[1 \mathbf{x}_{i}^{\top}\right]^{\top}, \boldsymbol{\gamma}$ é um vetor de parâmetros, $V_{k}$ é o efeito aleatório não observável da $k$-ésima clínica determinando a proporção de curados, sendo considerado que $V_{1}, \ldots, V_{M}$ são independentes com $V_{k} \sim N\left(0, \sigma_{V}^{2}\right)$. Note que valores maiores de $V_{k}$ indicam que aqueles pacientes em tratamento pela $k$-ésima clínica terão uma probabilidade maior de cura.

Para $k=1,2, \ldots, M$ e $j=1,2, \ldots, n_{k}$, conforme apresentado na seção 1.1, para o modelo de riscos proporcionais de Cox, tem-se

$$
h_{2}\left(t_{j k} ; \mathbf{x}_{j k}\right)=h_{20}\left(t_{j k}\right) \cdot e^{\mathbf{x}_{j k}^{\top} \boldsymbol{\beta}}
$$

em que $\boldsymbol{\beta}$ é o vetor de parâmetros da regressão e $h_{20}(t)$ é a função de riscos basal para os indivíduos não curados.

Neste trabalho, vamos supor que a função de risco basal para os não curados segue conforme a 
distribuição Weibull dada por

$$
h_{20}\left(t_{j k}\right)=\lambda \alpha\left(t_{j k}\right)^{\alpha-1}
$$

em que $\alpha>0$ e $\lambda>0$.

Para se determinar a verossimilhança, considere $C_{j k}$ o tempo de censura (à direita) para o $j$-ésimo indivíduo da clínica $k$. Seja $Y_{j k}=\min \left\{T_{j k}, C_{j k}\right\}$ e seja $\nu_{j k}$ o indicador de falha, ou seja, $\nu_{j k}=1$ se $y_{j k}$ é falha e 0 se for censura.

Assim, os dados observados são $D_{o b s}=(\mathbf{y}, \boldsymbol{\nu}, \mathbf{X})$ em que $\mathbf{y}=\left(y_{j k}\right)_{j=1, \ldots, n_{k}}^{k=1, \ldots, M}, \boldsymbol{\nu}=\left(\nu_{j k}\right)_{j=1, \ldots, n_{k}}^{k=1, \ldots, M}$ e $\mathbf{X}$ são as covariáveis.

Os dados completos são $D_{c}=(\mathbf{y}, \boldsymbol{\nu}, \mathbf{V}, \mathbf{X})$ que incluem os dados observados e os dados não observados $\mathbf{V}=\left[V_{1}, V_{2}, \ldots, V_{M}\right]$. O vetor de parâmetros é dado por $\boldsymbol{\phi}=\left(\alpha, \lambda, \boldsymbol{\beta}^{\top}, \boldsymbol{\gamma}^{\top}, \sigma_{V}^{2}\right)$.

Assim, utilizando (1.1), temos

$$
\begin{aligned}
f(\mathbf{y}, \mathbf{V})= & f(\mathbf{y} \mid \mathbf{V}) \cdot f(\mathbf{V}) \\
= & \prod_{k=1}^{M} \prod_{j=1}^{n_{k}}\left\{S\left(y_{j k} ; \mathbf{x}_{j k}\right)^{1-\nu_{j k}}\left(\pi\left(\mathbf{x}_{j k}\right) h_{20}\left(y_{j k}\right) e^{\mathbf{x}_{j k}^{\top} \boldsymbol{\beta}} S_{20}\left(y_{j k}\right)^{\exp \left(\mathbf{x}_{j k}^{\top} \boldsymbol{\beta}\right)}\right)^{\nu_{j k}}\right\} . \\
& \cdot \prod_{k=1}^{M}\left\{\frac{1}{\sqrt{2 \pi \sigma_{V}^{2}}} e^{\frac{-V_{k}^{2}}{2 \sigma_{V}^{2}}}\right\}
\end{aligned}
$$

De modo que a log-verossimilhança completa é dada por

$$
\begin{aligned}
l\left(\boldsymbol{\phi} \mid D_{c}\right) & =\sum_{k=1}^{M} \sum_{j=1}^{n_{k}}\left\{\left(1-\nu_{j k}\right) \ln \left(S\left(y_{j k} ; \mathbf{x}_{j k}\right)\right)+\nu_{j k} \ln \left(\pi\left(\mathbf{x}_{j k}\right) h_{20}\left(y_{j k}\right) e^{\mathbf{x}_{j k}^{\top} \boldsymbol{\beta}} S_{20}\left(y_{j k}\right)^{\exp \left(\mathbf{x}_{j k}^{\top} \boldsymbol{\beta}\right)}\right)\right\}+ \\
& +\sum_{k=1}^{M}\left\{\frac{-M}{2} \ln \left(2 \pi \sigma_{V}^{2}\right)-\frac{V_{k}^{2}}{2 \sigma_{V}^{2}}\right\}
\end{aligned}
$$

\subsection{Inferência bayesiana}

Com já afirmado anteriormente, na abordagem bayesiana, a presença de quantidades não observáveis não representa um problema de inferência já que toda e qualquer quantidade desconhecida é uma variável aleatória. Assim, tanto para as quantidades de interesse (parâmetros), quanto para os efeitos aleatórios, é necessário obter sua distribuição a posteriori, ou seja, condicional aos dados observados. Basicamente, a distribuição a posteriori é proporcional ao produto da distribuição a priori para as quantidades desconhecidas e da função de verossimilhança.

Para o modelo de mistura com efeitos aleatórios definido na seção 5.1, supondo prioris não 
informativas para $\boldsymbol{\gamma}$ e para $\boldsymbol{\beta}$, consideremos as distribuições a priori para o vetor de parâmetros $\phi$ :

$$
\pi(\phi)=\pi\left(\alpha, \lambda, \boldsymbol{\beta}^{\top}, \boldsymbol{\gamma}^{\top}, \sigma_{V}^{2}\right) \propto \pi(\alpha) \pi(\lambda) \pi\left(\sigma_{V}^{2}\right)
$$

em que as distribuições a priori são tais que

1. $\alpha \sim \operatorname{Gama}\left(\delta_{0}, \tau_{0}\right)$

$$
\pi(\alpha) \propto \alpha^{\delta_{0}-1} e^{-\tau_{0} \alpha}
$$

2. $\lambda \sim$ Gama Inversa $(a, b)$

$$
\pi(\lambda) \propto(\lambda)^{-(a+1)} e^{-\frac{b}{\lambda}}
$$

3. $\sigma_{V}^{2} \sim$ Gama Inversa $\left(a_{V}, b_{V}\right)$

$$
\pi\left(\sigma_{V}^{2}\right) \propto\left(\sigma_{V}^{2}\right)^{-\left(a_{V}+1\right)} e^{-\frac{b_{V}}{\sigma_{V}^{2}}}
$$

Considerando a função de verossimilhança em (5.2), a distribuição a posteriori é dada por

$$
\begin{aligned}
\pi\left(\boldsymbol{\phi}, \mathbf{V} \mid D_{o b s}\right)= & \prod_{k=1}^{M} \prod_{j=1}^{n_{k}}\left\{S\left(y_{j k} ; \mathbf{x}_{j k}\right)^{1-\nu_{j k}}\left(\pi\left(\mathbf{x}_{j k}\right) h_{20}\left(y_{j k}\right) e^{\mathbf{x}_{j k}^{\top} \boldsymbol{\beta}} S_{20}\left(y_{j k}\right)^{\exp \left(\mathbf{x}_{j k}^{\top} \boldsymbol{\beta}\right)}\right)^{\nu_{j k}}\right\} . \\
& \cdot \prod_{k=1}^{M}\left\{\frac{1}{\sqrt{2 \pi \sigma_{V}^{2}}} e^{\frac{-V_{k}^{2}}{2 \sigma_{V}^{2}}}\right\} \\
& \cdot \alpha^{\delta_{0}-1} e^{-\tau_{0} \alpha}(\lambda)^{-(a+1)} e^{-\frac{b}{\lambda}}\left(\sigma_{V}^{2}\right)^{-\left(a_{V}+1\right)} e^{-\frac{b_{V}}{\sigma_{V}^{2}}}
\end{aligned}
$$

Uma forma de se obter estimativas dos parâmetros seria via utilização de amostrador de Gibbs (Geman; Geman, 1984), método de simulação MCMC para aproximar as distribuições conjuntas e marginais, amostrando das distribuições condicionais. Assim, o próximo passo seria tentar identificar as distribuições condicionais, a partir de (5.4). Do mesmo modo que no modelo de Chen-IbrahimSinha com efeitos aleatórios, nem todas as distribuições condicionais terão forma fechada. Novamente, a alternativa escolhida é utilização de "Metropolis-Hastings"(Chib \& Greenberg, 1995).

Para a verificação da adequação da convergência do modelo, serão calculados os valores de $\sqrt{\hat{R}}$, já definido na seção 2.2, lembrando que valores de $\sqrt{\hat{R}}$ próximos a 1 para todas as estimativas de interesse indicam que houve convergência das cadeias e que foi atingida a distribuição de cada estimador. 


\subsection{Análise clássica}

Devido à presença de quantidades não observadas, dadas pelos efeitos aleatórios $\mathbf{V}$,a equação (5.2) não é exatamente uma verossimilhança. O modo escolhido para se encontrar estimadores para os parâmetros se baseia em primeiro calcular o "melhor preditor linear não-viesado"(BLUP) e estendêlo para se obter estimadores de máxima verossimilhança residual (REML) aproximados, como já ocorrido no modelo de Chen-Ibrahim-Sinha com efeitos aleatórios.

Para se encontrar o BLUP, considere:

- $\boldsymbol{\phi}_{E}=\left(\alpha, \lambda, \boldsymbol{\beta}^{\top}, \boldsymbol{\gamma}^{\top}, \sigma_{V}^{2}, \mathbf{V}^{\top}\right)^{\top}$;

- $l$ o logaritmo neperiano de (5.2), ou seja, a expressão dada em (5.3);

- U o vetor escore de $l$ em $\phi_{E}$, ou seja, o vetor das primeiras derivadas de $l$ em relação à $\phi_{E}$;

- $H$ a matriz hessiana de $l$ em $\phi_{E}$, ou seja, a matriz das segundas derivadas de $l$ em relação à $\phi_{E}$.

O BLUP para $\mathbf{V}$ e estimadores para $\phi$ serão encontrados a partir das raízes do vetor escore $\mathbf{U}$.

Lembre-se que $l$ é dada por

$$
\begin{aligned}
l\left(\boldsymbol{\phi} \mid D_{c}\right) & =\sum_{k=1}^{M} \sum_{j=1}^{n_{k}}\left\{\left(1-\nu_{j k}\right) \ln \left(S\left(y_{j k} ; \mathbf{x}_{j k}\right)\right)+\nu_{j k} \ln \left(\pi\left(\mathbf{x}_{j k}\right) h_{20}\left(y_{j k}\right) e^{\mathbf{x}_{j k}^{\top} \boldsymbol{\beta}} S_{20}\left(y_{j k}\right)^{\exp \left(\mathbf{x}_{j k}^{\top} \boldsymbol{\beta}\right)}\right)\right\}+ \\
& +\sum_{k=1}^{M}\left\{\frac{-M}{2} \ln \left(2 \pi \sigma_{V}^{2}\right)-\frac{V_{k}^{2}}{2 \sigma_{V}^{2}}\right\} .
\end{aligned}
$$

O vetor escore $\mathbf{U}$ é dado por $\mathbf{U}=\left(\frac{\partial l}{\partial \alpha}, \frac{\partial l}{\partial \lambda}, \frac{\partial l}{\partial \boldsymbol{\beta}^{\top}}, \frac{\partial l}{\partial \boldsymbol{\gamma}^{\top}}, \frac{\partial l}{\partial \sigma_{V}^{2}}, \frac{\partial l}{\partial \mathbf{V}^{\top}}\right)^{\top}$.

Para se encontrar o BLUP, deve-se encontrar a solução de $\mathbf{U}=\overrightarrow{0}$. Mas, isto não é de fácil solução.

Entretanto, note que a derivada de $l$ em $\sigma_{V}^{2}$ é dada por

$$
\begin{aligned}
\frac{\partial l}{\partial \sigma_{V}^{2}} & =\sum_{k=1}^{M}\left\{\frac{-1}{2} \frac{1}{\sigma_{V}^{2}}-\frac{V_{k}^{2}}{2}\left(\frac{-1}{\left(\sigma_{V}^{2}\right)^{2}}\right)\right\}=\sum_{k=1}^{M}\left\{\frac{-1}{2 \sigma_{V}^{2}}+\frac{V_{k}^{2}}{2\left(\sigma_{V}^{2}\right)^{2}}\right\}=\frac{1}{2 \sigma_{V}^{2}} \sum_{k=1}^{M}\left\{-1+\frac{V_{k}^{2}}{\sigma_{V}^{2}}\right\} \\
& =\frac{1}{2 \sigma_{V}^{2}}\left\{\sum_{k=1}^{M}(-1)+\frac{1}{\sigma_{V}^{2}} \sum_{k=1}^{M} V_{k}^{2}\right\}=\frac{1}{2 \sigma_{V}^{2}}\left\{-M+\frac{1}{\sigma_{V}^{2}} \sum_{k=1}^{M} V_{k}^{2}\right\}=\frac{-M \sigma_{V}^{2}+\sum_{k=1}^{M} V_{k}^{2}}{2 \sigma_{V}^{2}}
\end{aligned}
$$


Portanto,

$$
\begin{aligned}
\frac{\partial l}{\partial \sigma_{V}^{2}} & =0 \Longleftrightarrow \frac{-M \sigma_{V}^{2}+\sum_{k=1}^{M} V_{k}^{2}}{2 \sigma_{V}^{2}}=0 \Longleftrightarrow-M \sigma_{V}^{2}+\sum_{k=1}^{M} V_{k}^{2}=0 \Longleftrightarrow \\
\sigma_{V}^{2} & =\frac{\sum_{k=1}^{M} V_{k}^{2}}{M}
\end{aligned}
$$

Como, analiticamente, as soluções das demais equações não são viáveis, uma alternativa é utilizar o método de Newton-Raphson, que já foi apresentado na seção 2.3 e é repetido aqui, para maior clareza do leitor.

Se $\phi_{E}(t+1)$ são as estimativas em $\phi_{E}$ no passo $t+1$, se $\phi_{E}(t)$ são as estimativas em $\phi_{E}$ no passo $t$, se $\mathbf{U}(t)$ é o vetor escore calculado em $\phi_{E}(t)$ e se $H(t)$ é a matriz hessiana calculada em $\phi_{E}(t)$, então

$$
\phi_{E}(t+1)=\phi_{E}(t)-H(t)^{-1} \cdot \mathbf{U}(t)
$$

em que (5.5) é inicializado com valores para $\phi_{E}$ e deve ser calculado até que $\left\|\phi_{E}(t+1)-\phi_{E}(t)\right\|<\varepsilon$, $\operatorname{com} \varepsilon$ suficientemente pequeno. Pode-se, por exemplo, usar $\varepsilon=10^{-3}$.

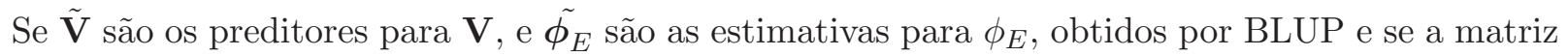
hessiana-inversa calculada em $\tilde{\phi_{E}}$ é decomposta relativamente a $\left(\alpha, \lambda, \boldsymbol{\beta}^{\top}, \boldsymbol{\gamma}^{\top}\right)\left|\sigma_{V}^{2}\right| \mathbf{V}^{\top}$ por

$$
H^{-1}=\left[\begin{array}{ccc}
A_{11} & A_{12} & A_{13} \\
A_{21} & A_{22} & A_{23} \\
A_{31} & A_{32} & A_{33}
\end{array}\right]
$$

então, segundo McGilchrist e Yau (1995) e Yau e Ng (2001), as estimativas para $\sigma_{V}^{2}$ via REML serão calculadas por

$$
\hat{\sigma_{V}^{2}}=\frac{1}{M}\left(-\operatorname{tr}\left(A_{33}\right)+\tilde{\mathbf{V}}^{\top} \tilde{\mathbf{V}}\right)
$$

A variância de $\hat{\sigma}_{V}^{2}$ é determinada por

$$
\operatorname{Var}\left(\hat{\sigma_{V}^{2}}\right)=2\left(\frac{1}{\sigma_{V}^{2}}\left(M+2 \frac{1}{\sigma_{V}^{2}} \operatorname{tr}\left(A_{33}\right)\right)-\frac{1}{\sigma_{V}^{4}} \operatorname{tr}\left(A_{33}^{2}\right) .\right)^{-1}
$$

As variâncias dos demais parâmetros são obtidas através da matriz informação de Fisher, ou seja, são dadas por

$$
\operatorname{Var}\left(\left[\alpha, \lambda, \boldsymbol{\beta}^{\top}, \boldsymbol{\gamma}^{\top}\right]^{\top}\right)=-\left[A_{11}\right]
$$




\section{Capítulo 6}

\section{Estudos de simulação - modelo de mistura com efeitos aleatórios}

Neste capítulo são apresentados um estudo de simulação feito para o modelo de mistura com efeitos aleatórios, apresentado no Capítulo 5, tanto para a abordagem bayesiana quanto para a clássica.

Para este estudo foram utilizados os programas R (versão 2.5.1), Winbugs (versão 1.4) e Maple (versão 10).

\subsection{Características}

Para este estudo, em uma primeira situação, estão assumidas as mesmas condições básicas apresentadas em Yau-Ng (2001) e que já foram apresentadas no Capítulo 3:

- $M=10$, ou seja, 10 clínicas;

- $n_{k}=15$ para todo $k=1,2, \ldots, M$, ou seja, cada clínica possui 15 indivíduos;

- em cada clínica, 5 pacientes são aleatoriamente designados para o grupo de tratamento $\left(x_{j k}=1\right)$ e os 10 restantes, para o grupo controle $\left(x_{j k}=0\right)$;

- 12 grupos de parâmetros a serem analisados:

1. $\left(\alpha=1 ; \sigma_{V}^{2}=0,5 ;\right.$ Censura $\left.=500 ; \beta_{1}=-0,5 ; \gamma_{0}=0 ; \gamma_{1}=0,5 ; \lambda=1\right)$,

2. $\left(\alpha=1,3 ; \sigma_{V}^{2}=0,5 ;\right.$ Censura $\left.=500 ; \beta_{1}=-0,5 ; \gamma_{0}=0 ; \gamma_{1}=0,5 ; \lambda=1\right)$,

3. $\left(\alpha=1 ; \sigma_{V}^{2}=1 ;\right.$ Censura $\left.=500 ; \beta_{1}=-0,5 ; \gamma_{0}=0 ; \gamma_{1}=0,5 ; \lambda=1\right)$,

4. $\left(\alpha=1 ; \sigma_{V}^{2}=0,5 ;\right.$ Censura $\left.=300 ; \beta_{1}=-0,5 ; \gamma_{0}=0 ; \gamma_{1}=0,5 ; \lambda=1\right)$,

5. $\left(\alpha=1 ; \sigma_{V}^{2}=0,5 ;\right.$ Censura $\left.=500 ; \beta_{1}=-0,5 ; \gamma_{0}=-0,5 ; \gamma_{1}=0,5 ; \lambda=1\right)$,

6. $\left(\alpha=1,3 ; \sigma_{V}^{2}=0,5 ;\right.$ Censura $\left.=500 ; \beta_{1}=-0,5 ; \gamma_{0}=-0,5 ; \gamma_{1}=0,5 ; \lambda=1\right)$,

7. $\left(\alpha=1 ; \sigma_{V}^{2}=1 ;\right.$ Censura $\left.=500 ; \beta_{1}=-0,5 ; \gamma_{0}=-0,5 ; \gamma_{1}=0,5 ; \lambda=1\right)$, 

8. $\left(\alpha=1 ; \sigma_{V}^{2}=0,5 ;\right.$ Censura $\left.=300 ; \beta_{1}=-0,5 ; \gamma_{0}=-0,5 ; \gamma_{1}=0,5 ; \lambda=1\right)$,
9. $\left(\alpha=1 ; \sigma_{V}^{2}=0,5 ;\right.$ Censura $\left.=500 ; \beta_{1}=-1 ; \gamma_{0}=-1 ; \gamma_{1}=1 ; \lambda=1\right)$,
10. $\left(\alpha=1,3 ; \sigma_{V}^{2}=0,5 ;\right.$ Censura $\left.=500 ; \beta_{1}=-1 ; \gamma_{0}=-1 ; \gamma_{1}=1 ; \lambda=1\right)$,
11. $\left(\alpha=1 ; \sigma_{V}^{2}=1 ;\right.$ Censura $\left.=500 ; \beta_{1}=-1 ; \gamma_{0}=-1 ; \gamma_{1}=1 ; \lambda=1\right)$,
12. $\left(\alpha=1 ; \sigma_{V}^{2}=0,5 ;\right.$ Censura $\left.=300 ; \beta_{1}=-1 ; \gamma_{0}=-1 ; \gamma_{1}=1 ; \lambda=1\right)$;

- 50 simulações para cada grupo de parâmetros.

Em um segundo momento foi estudado o caso em $n_{k}=50$ para todo $k=1,2, \ldots, 15$ com as demais condições mantidas.

O que se espera é que:

- o desempenho das estimativas melhore, quando $\alpha$ aumente de 1 para 1,3 , porque quanto menor o valor de $\alpha$, maior será a média da distribuição;

- uma diminuição na censura (de 500 para 300) piore o desempenho das estimativas, já que esta diminuição poderá fazer com que se tenha um aumento de indivíduos não curados que formam censurados;

- um aumento em $\sigma_{V}^{2}$ (de 0,5 para 1) produza uma maior variabilidade entre as clínicas, acarretando estimativas não tão boas quanto as obtidas para valores menores de $\sigma_{V}^{2}$;

- o aumento no número de indivíduos por clínica (de 15 para 50) melhore a acurácia dos resultados assintóticos.

\subsection{Simulação - abordagem bayesiana}

Em um primeiro momento foi feito um estudo de simulação com a utilização do programa Winbugs, versão 1.4. Os comando utilizados encontram-se no apêndice A.5. Os dados utilizados foram gerados com a utilização do programa R e encontram-se no apêndice A.4.

Os dados foram compilados sempre a partir de três cadeias iniciadas em valores distintos, com 30 mil iterações cada, seguindo o mesmo padrão já apresentado no Capítulo 3 para o modelo de Chen-Ibrahim-Sinha com efeitos aleatórios.

\subsubsection{Caso $n_{k}=15$}

Os resultados obtidos estão na Tabela 6.1, em que a terceira coluna apresenta a média das estimativas obtidas nas 50 simulações. Na quarta coluna está o desvio padrão calculado a partir dos 50 valores de estimativas de cada parâmetro. E, a quinta coluna contém a média dos desvios-padrão encontrados em cada uma das 50 simulações. 
Tabela 6.1: Resultado de 50 simulações via Metropolis-Hastings para $n_{k}=15$ para o modelo de mistura

\begin{tabular}{|c|c|c|c|c|}
\hline \multicolumn{5}{|c|}{ Grupo 1: $\left(\alpha=1 ; \sigma_{V}^{2}=0,5 ;\right.$ Censura $\left.=500 ; \beta_{1}=-0,5 ; \gamma_{0}=0 ; \gamma_{1}=0,5 ; \lambda=1\right)$} \\
\hline Parâmetro & Valor verdadeiro & Média da estimativa & DP da estimativa & Média dos DPs \\
\hline$\beta_{1}$ & $-0,5$ & $-0,4915$ & 0,2267 & 0,2234 \\
\hline$\gamma_{0}$ & 0 & $-0,0074$ & 0,3056 & 0,2352 \\
\hline$\gamma_{1}$ & 0,5 & 0,3519 & 0,3561 & 0,3599 \\
\hline$\sigma_{V}^{2}$ & 0,5 & 0,2645 & 0,0796 & 0,1481 \\
\hline \multicolumn{5}{|c|}{ Grupo 2: $\left(\alpha=1,3 ; \sigma_{V}^{2}=0,5 ;\right.$ Censura $\left.=500 ; \beta_{1}=-0,5 ; \gamma_{0}=0 ; \gamma_{1}=0,5 ; \lambda=1\right)$} \\
\hline Parâmetro & Valor verdadeiro & Média da estimativa & DP da estimativa & Média dos DPs \\
\hline$\beta_{1}$ & $-0,5$ & $-0,5375$ & 0,2572 & 0,2454 \\
\hline$\gamma_{0}$ & 0 & 0,0475 & 0,2794 & 0,2340 \\
\hline$\gamma_{1}$ & 0,5 & 0,5915 & 0,3927 & 0,3695 \\
\hline$\sigma_{V}^{2}$ & 0,5 & 0,2570 & 0,0687 & 0,1429 \\
\hline \multicolumn{5}{|c|}{ Grupo 3: $\left(\alpha=1 ; \sigma_{V}^{2}=1 ;\right.$ Censura $\left.=500 ; \beta_{1}=-0,5 ; \gamma_{0}=0 ; \gamma_{1}=0,5 ; \lambda=1\right)$} \\
\hline Parâmetro & Valor verdadeiro & Média da estimativa & DP da estimativa & Média dos DPs \\
\hline$\beta_{1}$ & $-0,5$ & $-0,5548$ & 0,2621 & 0,2326 \\
\hline$\gamma_{0}$ & 0 & 0,0097 & 0,3242 & 0,2489 \\
\hline$\gamma_{1}$ & 0,5 & 0,4804 & 0,3751 & 0,3677 \\
\hline$\sigma_{V}^{2}$ & 1 & 0,3769 & 0,2027 & 0,2351 \\
\hline \multicolumn{5}{|c|}{ Grupo 4: $\left(\alpha=1 ; \sigma_{V}^{2}=0,5 ;\right.$ Censura $\left.=300 ; \beta_{1}=-0,5 ; \gamma_{0}=0 ; \gamma_{1}=0,5 ; \lambda=1\right)$} \\
\hline Parâmetro & Valor verdadeiro & Média da estimativa & DP da estimativa & Média dos DPs \\
\hline$\beta_{1}$ & $-0,5$ & $-0,5048$ & 0,2446 & 0,2320 \\
\hline$\gamma_{0}$ & 0 & $-0,0033$ & 0,2721 & 0,2379 \\
\hline$\gamma_{1}$ & 0,5 & 0,4787 & 0,3635 & 0,3651 \\
\hline$\sigma_{V}^{2}$ & 0,5 & 0,2897 & 0,1127 & 0,1681 \\
\hline \multicolumn{5}{|c|}{ Grupo $5:\left(\alpha=1 ; \sigma_{V}^{2}=0,5 ;\right.$ Censura $\left.=500 ; \beta_{1}=-0,5 ; \gamma_{0}=-0,5 ; \gamma_{1}=0,5 ; \lambda=1\right)$} \\
\hline Parâmetro & Valor verdadeiro & Média da estimativa & DP da estimativa & Média dos DPs \\
\hline$\beta_{1}$ & $-0,5$ & $-0,5352$ & 0,1955 & 0,2061 \\
\hline$\gamma_{0}$ & $-0,5$ & $-0,4479$ & 0,3034 & 0,2401 \\
\hline$\gamma_{1}$ & 0,5 & 0,5038 & 0,3138 & 0,3598 \\
\hline$\sigma_{V}^{2}$ & 0,5 & 0,2690 & 0,0789 & 0,1527 \\
\hline
\end{tabular}




\begin{tabular}{|c|c|c|c|c|}
\hline \multicolumn{5}{|c|}{ Grupo 6: $\left(\alpha=1,3 ; \sigma_{V}^{2}=0,5 ;\right.$ Censura $\left.=500 ; \beta_{1}=-0,5 ; \gamma_{0}=-0,5 ; \gamma_{1}=0,5 ; \lambda=1\right)$} \\
\hline Parâmetro & Valor verdadeiro & Média da estimativa & DP da estimativa & Média dos DPs \\
\hline$\beta_{1}$ & $-0,5$ & $-0,5071$ & 0,2085 & 0,2054 \\
\hline$\gamma_{0}$ & $-0,5$ & $-0,4296$ & 0,3422 & 0,2371 \\
\hline$\gamma_{1}$ & 0,5 & 0,4576 & 0,3863 & 0,3592 \\
\hline$\sigma_{V}^{2}$ & 0,5 & 0,2394 & 0,0550 & 0,1276 \\
\hline \multicolumn{5}{|c|}{ Grupo $7:\left(\alpha=1 ; \sigma_{V}^{2}=1 ;\right.$ Censura $\left.=500 ; \beta_{1}=-0,5 ; \gamma_{0}=-0,5 ; \gamma_{1}=0,5 ; \lambda=1\right)$} \\
\hline Parâmetro & Valor verdadeiro & Média da estimativa & DP da estimativa & Média dos DPs \\
\hline$\beta_{1}$ & $-0,5$ & $-0,4753$ & 0,2108 & 0,2018 \\
\hline$\gamma_{0}$ & $-0,5$ & $-0,3909$ & 0,3245 & 0,2516 \\
\hline$\gamma_{1}$ & 0,5 & 0,3354 & 0,3991 & 0,3651 \\
\hline$\sigma_{V}^{2}$ & 1 & 0,3769 & 0,2021 & 0,2353 \\
\hline \multicolumn{5}{|c|}{ Grupo 8: $\left(\alpha=1 ; \sigma_{V}^{2}=0,5 ;\right.$ Censura $\left.=300 ; \beta_{1}=-0,5 ; \gamma_{0}=-0,5 ; \gamma_{1}=0,5 ; \lambda=1\right)$} \\
\hline Parâmetro & Valor verdadeiro & Média da estimativa & DP da estimativa & Média dos DPs \\
\hline$\beta_{1}$ & $-0,5$ & $-0,5054$ & 0,1912 & 0,2003 \\
\hline$\gamma_{0}$ & $-0,5$ & $-0,4829$ & 0,2451 & 0,2415 \\
\hline$\gamma_{1}$ & 0,5 & 0,4115 & 0,2892 & 0,3605 \\
\hline$\sigma_{V}^{2}$ & 0,5 & 0,2834 & 0,1225 & 0,1619 \\
\hline \multicolumn{5}{|c|}{ Grupo 9: $\left(\alpha=1 ; \sigma_{V}^{2}=0,5 ;\right.$ Censura $\left.=500 ; \beta_{1}=-1 ; \gamma_{0}=-1 ; \gamma_{1}=1 ; \lambda=1\right)$} \\
\hline Parâmetro & Valor verdadeiro & Média da estimativa & DP da estimativa & Média dos DPs \\
\hline$\beta_{1}$ & -1 & $-1,0252$ & 0,2245 & 0,2045 \\
\hline$\gamma_{0}$ & -1 & $-0,8421$ & 0,3188 & 0,2537 \\
\hline$\gamma_{1}$ & 1 & 0,8655 & 0,3110 & 0,3679 \\
\hline$\sigma_{V}^{2}$ & 0,5 & 0,2893 & 0,1789 & 0,1683 \\
\hline \multicolumn{5}{|c|}{ Grupo 10: $\left(\alpha=1,3 ; \sigma_{V}^{2}=0,5 ;\right.$ Censura $\left.=500 ; \beta_{1}=-1 ; \gamma_{0}=-1 ; \gamma_{1}=1 ; \lambda=1\right)$} \\
\hline Parâmetro & Valor verdadeiro & Média da estimativa & DP da estimativa & Média dos DPs \\
\hline$\beta_{1}$ & -1 & $-0,9669$ & 0,2247 & 0,2021 \\
\hline$\gamma_{0}$ & -1 & $-0,9506$ & 0,3294 & $-0,2528$ \\
\hline$\gamma_{1}$ & 1 & 0,9116 & 0,4410 & 0,3701 \\
\hline$\sigma_{V}^{2}$ & 0,5 & 0,2430 & 0,0556 & 0,1321 \\
\hline \multicolumn{5}{|c|}{ Grupo 11: $\left(\alpha=1 ; \sigma_{V}^{2}=1 ;\right.$ Censura $\left.=500 ; \beta_{1}=-1 ; \gamma_{0}=-1 ; \gamma_{1}=1 ; \lambda=1\right)$} \\
\hline Parâmetro & Valor verdadeiro & Média da estimativa & DP da estimativa & Média dos DPs \\
\hline$\beta_{1}$ & -1 & $-0,9903$ & 0,2395 & 0,2028 \\
\hline$\gamma_{0}$ & -1 & $-0,8847$ & 0,3715 & 0,2702 \\
\hline$\gamma_{1}$ & 1 & 0,8690 & 0,3170 & 0,3752 \\
\hline$\sigma_{V}^{2}$ & 1 & 0,4129 & 0,2366 & 0,2661 \\
\hline \multicolumn{5}{|c|}{ Grupo 12: $\left(\alpha=1 ; \sigma_{V}^{2}=0,5 ;\right.$ Censura $\left.=300 ; \beta_{1}=-1 ; \gamma_{0}=-1 ; \gamma_{1}=1 ; \lambda=1\right)$} \\
\hline Parâmetro & Valor verdadeiro & Média da estimativa & DP da estimativa & Média dos DPs \\
\hline$\beta_{1}$ & -1 & $-1,0042$ & 0,2149 & 0,2055 \\
\hline$\gamma_{0}$ & -1 & $-0,9379$ & 0,3115 & 0,2525 \\
\hline$\gamma_{1}$ & 1 & 0,9779 & 0,4146 & 0,3698 \\
\hline$\sigma_{V}^{2}$ & 0,5 & 0,2459 & 0,0624 & 0,1346 \\
\hline
\end{tabular}

Note que a diferença entre os valores médios estimados estão próximos dos valores verdadeiros, principalmente levando-se em consideração os valores dos desvios-padrão, o que indica que os resultados obtidos via simulação estão razoalvemente bons. Note que os valores estimados para $\sigma_{V}^{2}$ são sempre menores que o seu correspondente valor verdadeiro, e que em alguns casos, a média das 
estimativa não está muito próxima do valor verdadeiro para $\sigma_{V}^{2}$, podendo ser um indício de que esta abordagem pode não ser muito boa.

As Figuras de 6.1 a 6.3 mostram os histogramas feitos a partir das estimativas calculadas nas 50 simulações, para cada um dos parâmetros.
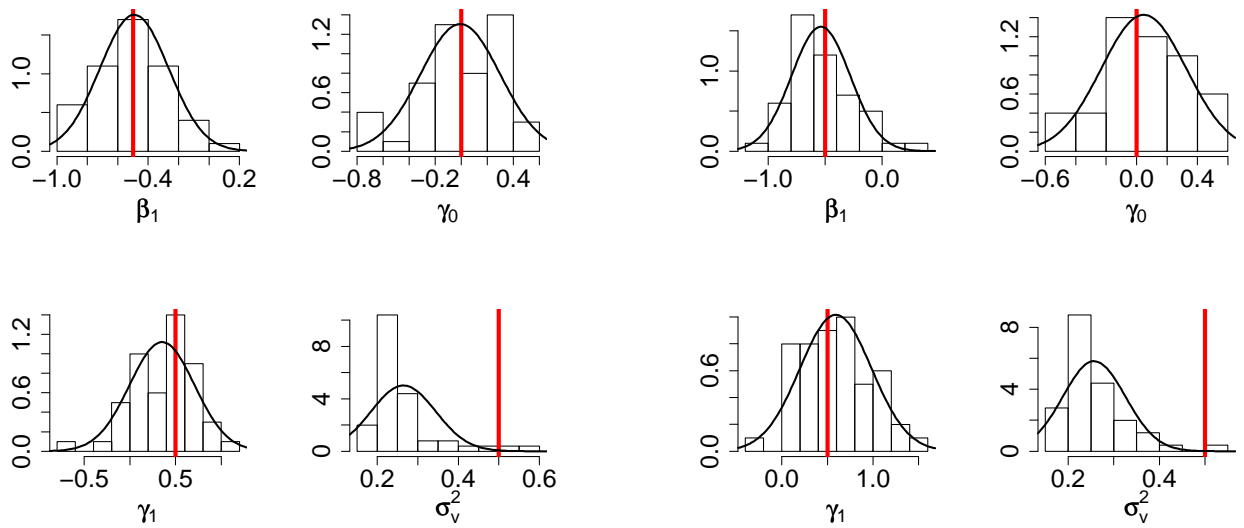

(a) Grupo 1

(b) Grupo 2
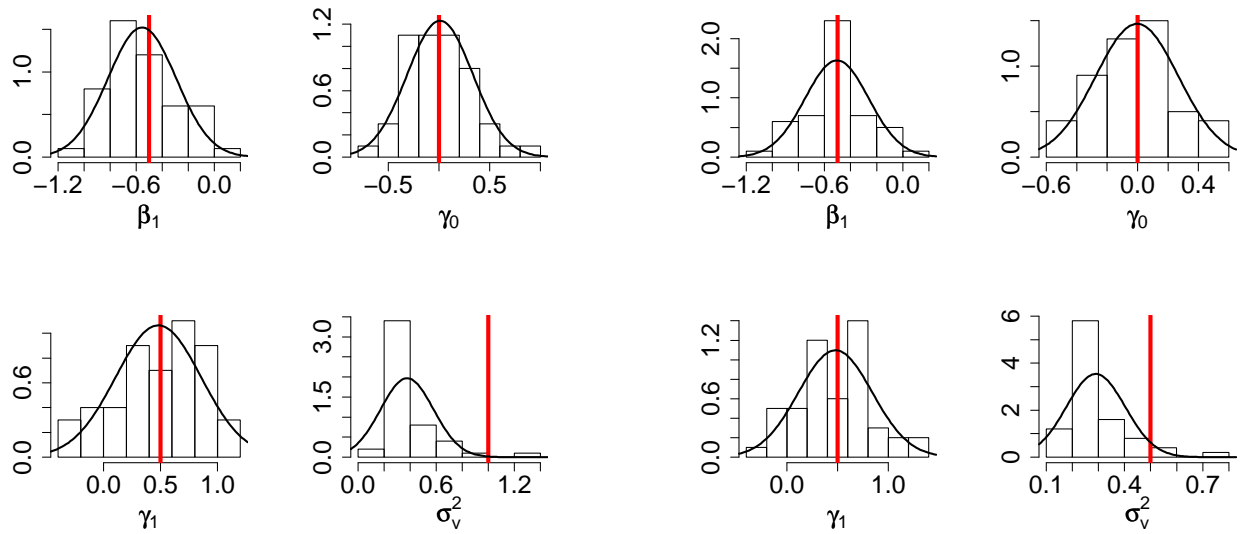

(c) Grupo 3
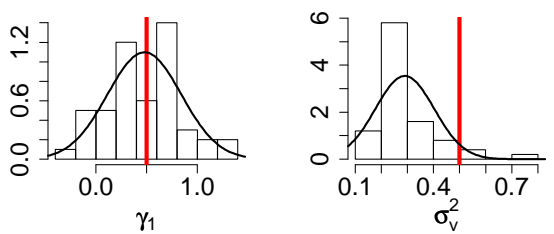

(d) Grupo 4

Figura 6.1: Histogramas das estimativas dos parâmetros estimados via Metropolis-Hastings $-n_{k}=15-$ modelo de mistura - parte 1 

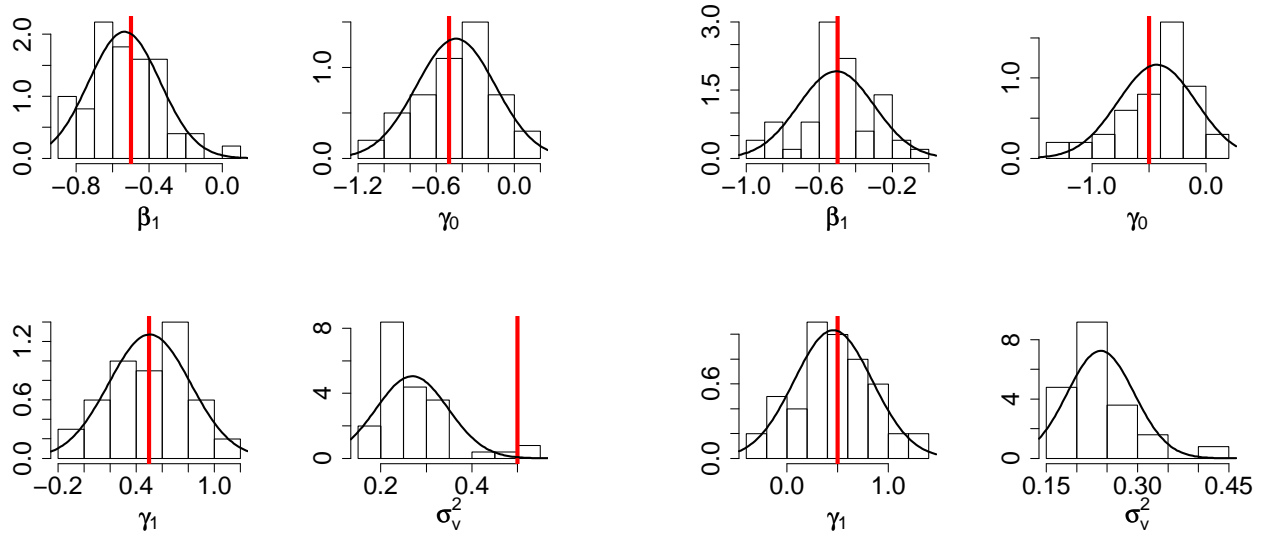

(a) Grupo 5

(b) Grupo 6
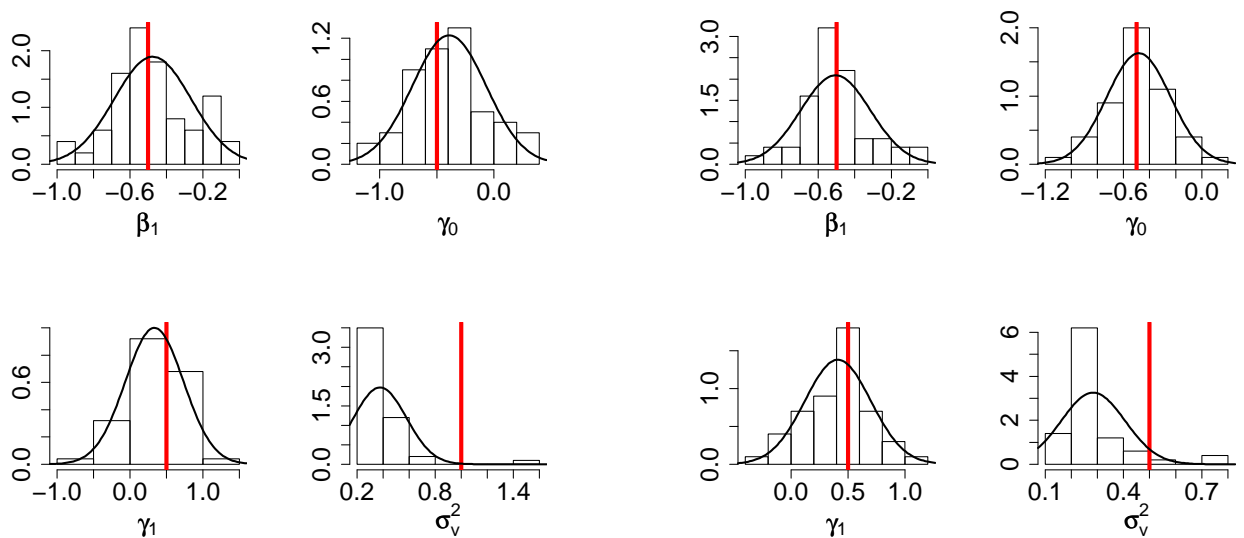

(c) Grupo 7

(d) Grupo 8

Figura 6.2: Histogramas das estimativas dos parâmetros estimados via Metropolis-Hastings $-n_{k}=15-$ modelo de mistura- parte 2 

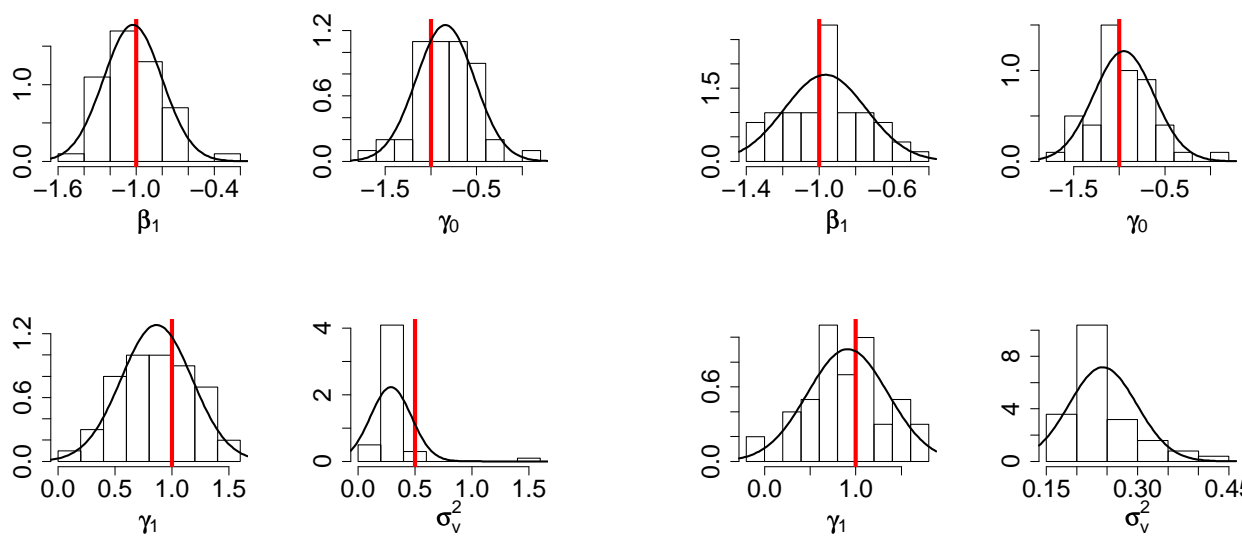

(a) Grupo 9
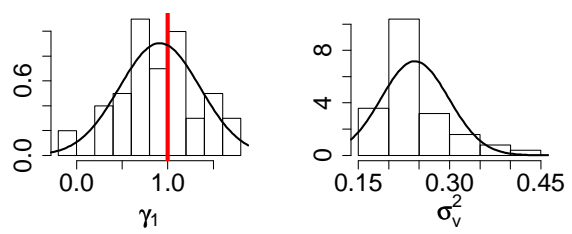

(b) Grupo 10
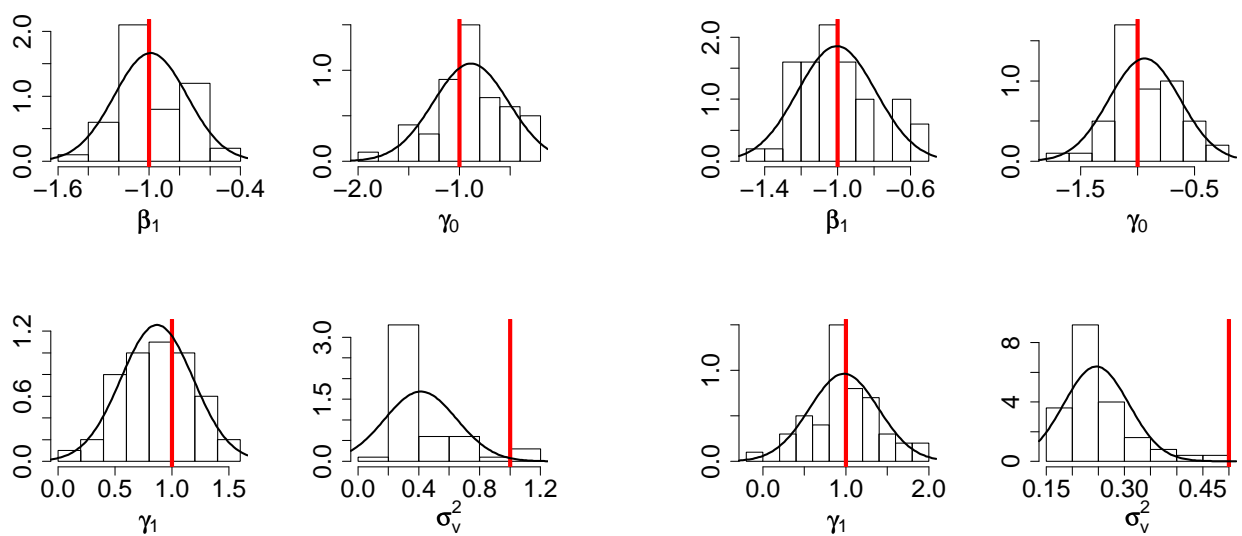

(c) Grupo 11
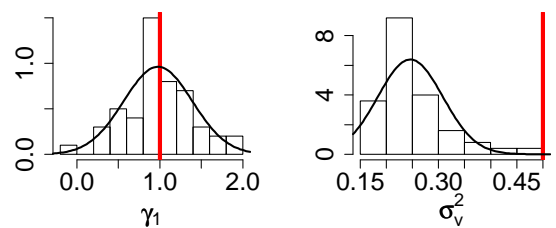

(d) Grupo 12

Figura 6.3: Histogramas das estimativas dos parâmetros estimados via Metropolis-Hastings $-n_{k}=15-$ modelo de mistura - parte 3

Estes histogramas indicam que as estimativas obtidas para $\beta_{1}, \gamma_{0}$ e $\gamma_{1}$ estão em torno dos valores verdadeiros, que estão representados pelas retas verticais. No caso de $\sigma_{V}^{2}$, há um indício de que os valores estão sendo subestimados.

Para se avaliar se a convergência do modelo está adequada, calcula-se os valores de $\sqrt{\hat{R}}$, como definido ao final da seção 2.2. Para isso, considere $m=3$ o número de cadeias utilizadas com valores iniciais distintos, em que cada uma apresenta $2 n=30000$ valores, ou seja, $n=15000$. Foram calculados os valores de $\sqrt{\hat{R}}$ para simulações em cada um dos 12 grupos. Os resultados obtidos encontram-se na Tabela 6.2. 
Tabela 6.2: Valores de $\sqrt{\hat{R}}-$ caso $n_{k}=15$ - modelo de mistura

\begin{tabular}{ccccccc}
\hline parâmetro & $\mathbf{1}$ & $\mathbf{2}$ & $\mathbf{3}$ & $\mathbf{4}$ & $\mathbf{5}$ & $\mathbf{6}$ \\
\hline$\beta_{1}$ & 1,00060 & 1,00028 & 1,00013 & 1,00008 & 1,00003 & 1,00017 \\
$\gamma_{0}$ & 1,00088 & 1,00158 & 1,00023 & 1,00011 & 1,00118 & 1,00046 \\
$\gamma_{1}$ & 1,00005 & 1,00087 & 1,00036 & 1,00005 & 1,00146 & 1,00041 \\
$\sigma_{V}^{2}$ & 1,00025 & 1,00090 & 1,00135 & 1,00195 & 1,00039 & 1,00039 \\
\hline \multicolumn{7}{c}{} \\
parâmetro & $\mathbf{7}$ & $\mathbf{8}$ & $\mathbf{9}$ & $\mathbf{1 0}$ & $\mathbf{1 1}$ & $\mathbf{1 2}$ \\
\hline$\beta_{1}$ & 1,00019 & 1,00023 & 1,00074 & 1,00009 & 1,00012 & 1,00010 \\
$\gamma_{0}$ & 1,00002 & 1,00082 & 1,00054 & 1,00156 & 1,00215 & 1,00219 \\
$\gamma_{1}$ & 1,00055 & 1,00088 & 1,00047 & 1,00213 & 1,00075 & 1,00093 \\
$\sigma_{V}^{2}$ & 1,00120 & 1,00248 & 1,00035 & 1,00103 & 1,00281 & 1,00034 \\
\hline
\end{tabular}

É perceptível que todos os valores estão bem próximos a 1 para todas as estimativas de interesse indicando que houve convergência da cadeia e que foi atingida a distribuição de cada estimador.

\subsubsection{Caso $n_{k}=50$}

Os resultados obtidos estão na Tabela 6.3, em que a terceira coluna apresenta a média das estimativas obtidas nas 50 simulações. Na quarta coluna está o desvio padrão calculado a partir dos 50 valores de estimativas de cada parâmetro. E, a quinta coluna contém a média dos desvios-padrão encontrados em cada uma das 50 simulações. 
Tabela 6.3: Resultado de 50 simulações via Metropolis-Hastings para $n_{k}=50$ para o modelo de mistura

\begin{tabular}{|c|c|c|c|c|}
\hline \multicolumn{5}{|c|}{ Grupo 1: $\left(\alpha=1 ; \sigma_{V}^{2}=0,5 ;\right.$ Censura $\left.=500 ; \beta_{1}=-0,5 ; \gamma_{0}=0 ; \gamma_{1}=0,5 ; \lambda=1\right)$} \\
\hline Parâmetro & Valor verdadeiro & Média da estimativa & DP da estimativa & Média dos DPs \\
\hline$\beta_{1}$ & $-0,5$ & $-0,5029$ & 0,1192 & 0,1245 \\
\hline$\gamma_{0}$ & 0 & 0,0070 & 0,2448 & 0,1790 \\
\hline$\gamma_{1}$ & 0,5 & 0,4848 & 0,1957 & 0,1996 \\
\hline$\sigma_{V}^{2}$ & 0,5 & 0,3889 & 0,1277 & 0,1746 \\
\hline \multicolumn{5}{|c|}{ Grupo 2: $\left(\alpha=1,3 ; \sigma_{V}^{2}=0,5 ;\right.$ Censura $\left.=500 ; \beta_{1}=-0,5 ; \gamma_{0}=0 ; \gamma_{1}=0,5 ; \lambda=1\right)$} \\
\hline Parâmetro & Valor verdadeiro & Média da estimativa & DP da estimativa & Média dos DPs \\
\hline$\beta_{1}$ & $-0,5$ & $-0,5240$ & 0,1434 & 0,1269 \\
\hline$\gamma_{0}$ & 0 & 0,0744 & 0,2255 & 0,1827 \\
\hline$\gamma_{1}$ & 0,5 & 0,4841 & 0,1963 & 0,2012 \\
\hline$\sigma_{V}^{2}$ & 0,5 & 0,4266 & 0,2389 & 0,1881 \\
\hline \multicolumn{5}{|c|}{ Grupo 3: $\left(\alpha=1 ; \sigma_{V}^{2}=1 ;\right.$ Censura $\left.=500 ; \beta_{1}=-0,5 ; \gamma_{0}=0 ; \gamma_{1}=0,5 ; \lambda=1\right)$} \\
\hline Parâmetro & Valor verdadeiro & Média da estimativa & DP da estimativa & Média dos DPs \\
\hline$\beta_{1}$ & $-0,5$ & $-0,4797$ & 0,1192 & 0,1228 \\
\hline$\gamma_{0}$ & 0 & $-0,0269$ & 0,3378 & 0,2216 \\
\hline$\gamma_{1}$ & 0,5 & 0,4873 & 0,1905 & 0,2065 \\
\hline$\sigma_{V}^{2}$ & 1 & 0,7425 & 0,3809 & 0,3222 \\
\hline \multicolumn{5}{|c|}{ Grupo $4:\left(\alpha=1 ; \sigma_{V}^{2}=0,5 ;\right.$ Censura $\left.=300 ; \beta_{1}=-0,5 ; \gamma_{0}=0 ; \gamma_{1}=0,5 ; \lambda=1\right)$} \\
\hline Parâmetro & Valor verdadeiro & Média da estimativa & DP da estimativa & Média dos DPs \\
\hline$\beta_{1}$ & $-0,5$ & $-0,4950$ & 0,1384 & 0,1246 \\
\hline$\gamma_{0}$ & 0 & $-0,0059$ & 0,2548 & 0,1856 \\
\hline$\gamma_{1}$ & 0,5 & 0,4976 & 0,1630 & 0,2007 \\
\hline$\sigma_{V}^{2}$ & 0,5 & 0,4361 & 0,1706 & 0,1953 \\
\hline \multicolumn{5}{|c|}{ Grupo $5:\left(\alpha=1 ; \sigma_{V}^{2}=0,5 ;\right.$ Censura $\left.=500 ; \beta_{1}=-0,5 ; \gamma_{0}=-0,5 ; \gamma_{1}=0,5 ; \lambda=1\right)$} \\
\hline Parâmetro & Valor verdadeiro & Média da estimativa & DP da estimativa & Média dos DPs \\
\hline$\beta_{1}$ & $-0,5$ & $-0,4916$ & 0,1097 & 0,1096 \\
\hline$\gamma_{0}$ & $-0,5$ & $-0,4421$ & 0,2599 & 0,1813 \\
\hline$\gamma_{1}$ & 0,5 & 0,4523 & 0,1915 & 0,1977 \\
\hline$\sigma_{V}^{2}$ & 0,5 & 0,3983 & 0,1571 & 0,1787 \\
\hline \multicolumn{5}{|c|}{ Grupo 6: $\left(\alpha=1,3 ; \sigma_{V}^{2}=0,5 ;\right.$ Censura $\left.=500 ; \beta_{1}=-0,5 ; \gamma_{0}=-0,5 ; \gamma_{1}=0,5 ; \lambda=1\right)$} \\
\hline Parâmetro & Valor verdadeiro & Média da estimativa & DP da estimativa & Média dos DPs \\
\hline$\beta_{1}$ & $-0,5$ & $-0,4979$ & 0,1179 & 0,1098 \\
\hline$\gamma_{0}$ & $-0,5$ & $-0,4696$ & 0,2345 & 0,1874 \\
\hline$\gamma_{1}$ & 0,5 & 0,4872 & 0,2021 & 0,1986 \\
\hline$\sigma_{V}^{2}$ & 0,5 & 0,4383 & 0,1998 & 0,1966 \\
\hline
\end{tabular}




\begin{tabular}{|c|c|c|c|c|}
\hline \multicolumn{5}{|c|}{ Grupo $7:\left(\alpha=1 ; \sigma_{V}^{2}=1 ;\right.$ Censura $\left.=500 ; \beta_{1}=-0,5 ; \gamma_{0}=-0,5 ; \gamma_{1}=0,5 ; \lambda=1\right)$} \\
\hline Parâmetro & Valor verdadeiro & Média da estimativa & DP da estimativa & Média dos DPs \\
\hline$\beta_{1}$ & $-0,5$ & $-0,4957$ & 0,1099 & 0,1115 \\
\hline$\gamma_{0}$ & $-0,5$ & $-0,4365$ & 0,2922 & 0,2232 \\
\hline$\gamma_{1}$ & 0,5 & 0,5192 & 0,1987 & 0,2052 \\
\hline$\sigma_{V}^{2}$ & 1 & 0,7532 & 0,4462 & 0,3244 \\
\hline \multicolumn{5}{|c|}{ Grupo 8: $\left(\alpha=1 ; \sigma_{V}^{2}=0,5 ;\right.$ Censura $\left.=300 ; \beta_{1}=-0,5 ; \gamma_{0}=-0,5 ; \gamma_{1}=0,5 ; \lambda=1\right)$} \\
\hline Parâmetro & Valor verdadeiro & Média da estimativa & DP da estimativa & Média dos DPs \\
\hline$\beta_{1}$ & $-0,5$ & $-0,4723$ & 0,1109 & 0,1079 \\
\hline$\gamma_{0}$ & $-0,5$ & $-0,5336$ & 0,2339 & 0,1851 \\
\hline$\gamma_{1}$ & 0,5 & 0,4759 & 0,1990 & 0,1986 \\
\hline$\sigma_{V}^{2}$ & 0,5 & 0,4195 & 0,1823 & 0,1894 \\
\hline \multicolumn{5}{|c|}{ Grupo 9: $\left(\alpha=1 ; \sigma_{V}^{2}=0,5 ;\right.$ Censura $\left.=500 ; \beta_{1}=-1 ; \gamma_{0}=-1 ; \gamma_{1}=1 ; \lambda=1\right)$} \\
\hline Parâmetro & Valor verdadeiro & Média da estimativa & DP da estimativa & Média dos DPs \\
\hline$\beta_{1}$ & -1 & $-0,9885$ & 0,1236 & 0,1100 \\
\hline$\gamma_{0}$ & -1 & $-0,9702$ & 0,2585 & 0,1888 \\
\hline$\gamma_{1}$ & 1 & 1,0073 & 0,2070 & 0,2046 \\
\hline$\sigma_{V}^{2}$ & 0,5 & 0,4014 & 0,1628 & 0,1836 \\
\hline \multicolumn{5}{|c|}{ Grupo 10: $\left(\alpha=1,3 ; \sigma_{V}^{2}=0,5 ;\right.$ Censura $\left.=500 ; \beta_{1}=-1 ; \gamma_{0}=-1 ; \gamma_{1}=1 ; \lambda=1\right)$} \\
\hline Parâmetro & Valor verdadeiro & Média da estimativa & DP da estimativa & Média dos DPs \\
\hline$\beta_{1}$ & -1 & $-0,9896$ & 0,1237 & 0,1096 \\
\hline$\gamma_{0}$ & -1 & $-0,9566$ & 0,2616 & $-0,1921$ \\
\hline$\gamma_{1}$ & 1 & 0,9566 & 0,2257 & 0,2054 \\
\hline$\sigma_{V}^{2}$ & 0,5 & 0,4296 & 0,2217 & 0,1975 \\
\hline \multicolumn{5}{|c|}{ Grupo 11: $\left(\alpha=1 ; \sigma_{V}^{2}=1 ;\right.$ Censura $\left.=500 ; \beta_{1}=-1 ; \gamma_{0}=-1 ; \gamma_{1}=1 ; \lambda=1\right)$} \\
\hline Parâmetro & Valor verdadeiro & Média da estimativa & DP da estimativa & Média dos DPs \\
\hline$\beta_{1}$ & -1 & $-1,0013$ & 0,1179 & 0,1096 \\
\hline$\gamma_{0}$ & -1 & $-0,9973$ & 0,3266 & 0,2416 \\
\hline$\gamma_{1}$ & 1 & 0,9934 & 0,2160 & 0,2141 \\
\hline$\sigma_{V}^{2}$ & 1 & 0,8491 & 0,4575 & 0,3723 \\
\hline \multicolumn{5}{|c|}{ Grupo 12: $\left(\alpha=1 ; \sigma_{V}^{2}=0,5 ;\right.$ Censura $\left.=300 ; \beta_{1}=-1 ; \gamma_{0}=-1 ; \gamma_{1}=1 ; \lambda=1\right)$} \\
\hline Parâmetro & Valor verdadeiro & Média da estimativa & DP da estimativa & Média dos DPs \\
\hline$\beta_{1}$ & -1 & $-0,9682$ & 0,0948 & 0,1084 \\
\hline$\gamma_{0}$ & -1 & $-0,9580$ & 0,2699 & 0,1884 \\
\hline$\gamma_{1}$ & 1 & 0,9214 & 0,2071 & 0,2040 \\
\hline$\sigma_{V}^{2}$ & 0,5 & 0,3949 & 0,1623 & 0,1815 \\
\hline
\end{tabular}

Note que a diferença entre os valores médios estimados e os valores verdadeiros são menores do que um desvio-padrão, em quase todos os casos, o que indica que os resultados obtidos via simulação estão bons.

Note ainda que as estimativas obtidas para este caso $n_{k}=50$ estão mais próximos dos valores verdadeiros do que no caso $n_{k}=15$.

As Figuras de 6.4 a 6.6 mostram os histogramas feitos a partir das estimativas calculadas nas 50 simulações, para cada um dos parâmetros. 

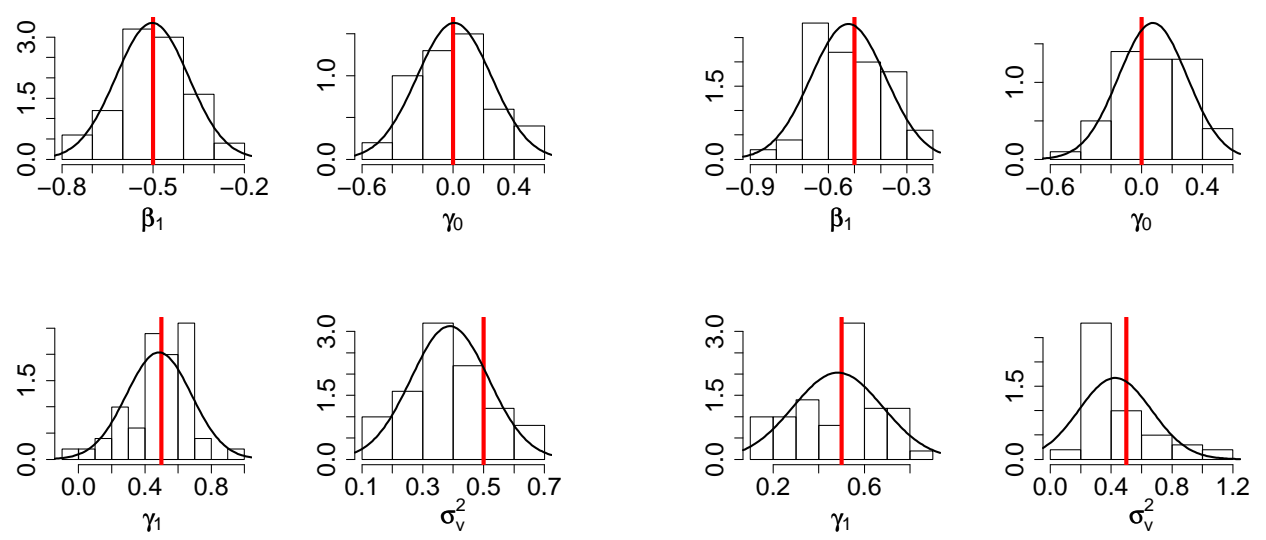

(a) Grupo 1

(b) Grupo 2
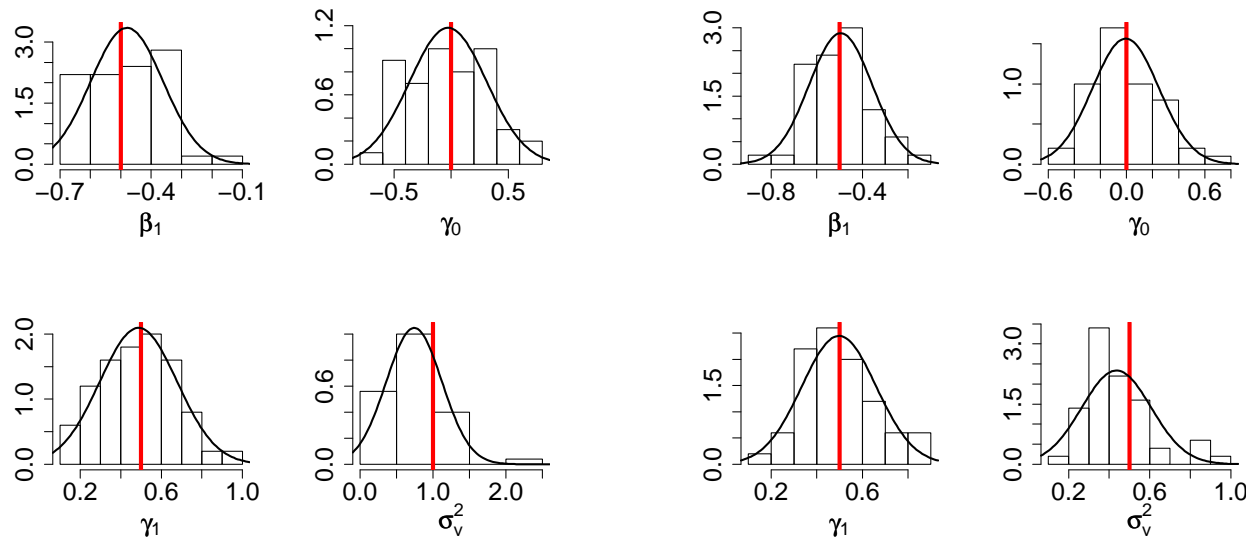

(c) Grupo 3

(d) Grupo 4

Figura 6.4: Histogramas das estimativas dos parâmetros estimados via Metropolis-Hastings $-n_{k}=50-$ modelo de mistura - parte 1 

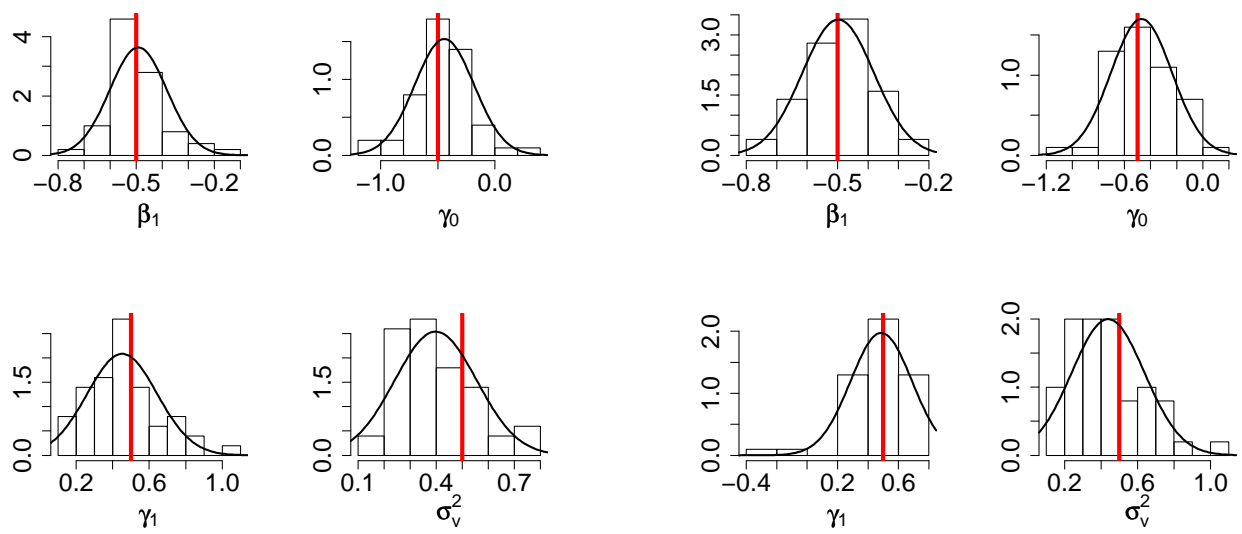

(a) Grupo 5

(b) Grupo 6
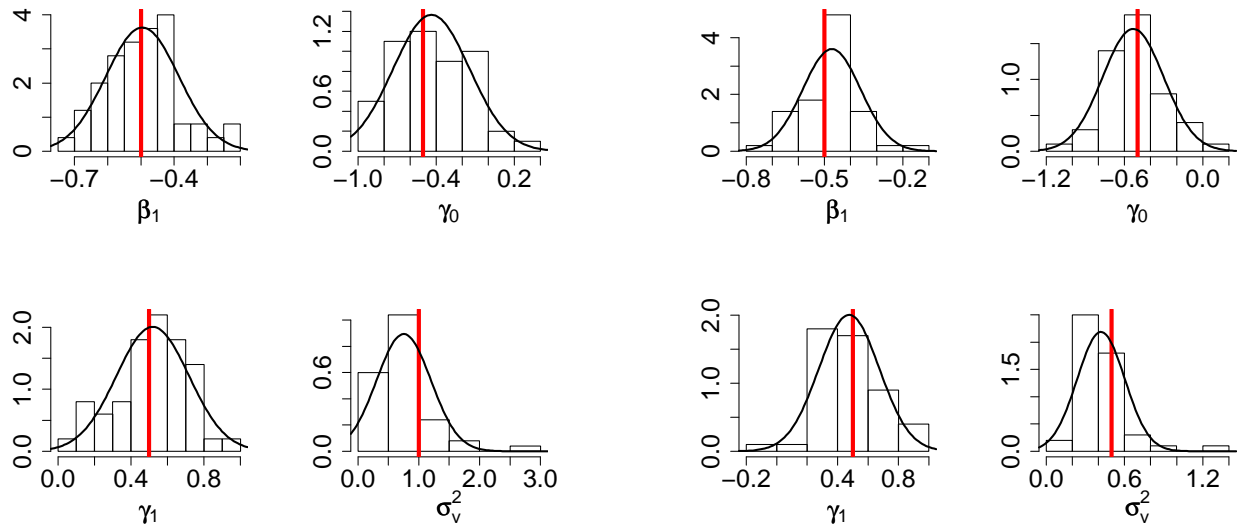

(c) Grupo 7
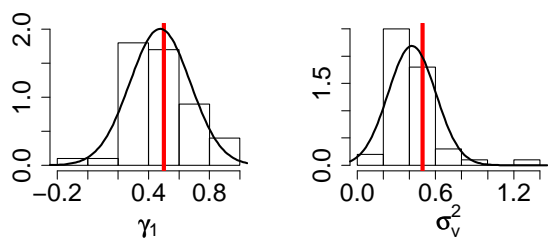

(d) Grupo 8

Figura 6.5: Histogramas das estimativas dos parâmetros estimados via Metropolis-Hastings $-n_{k}=50-$ modelo de mistura - parte 2 

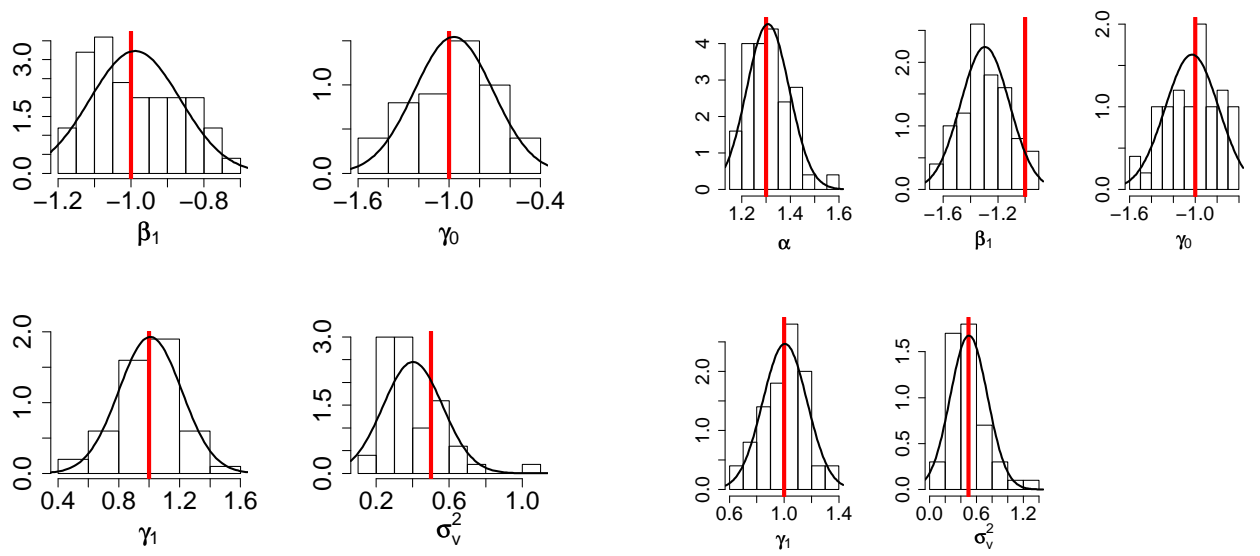

(a) Grupo 9

(b) Grupo 10
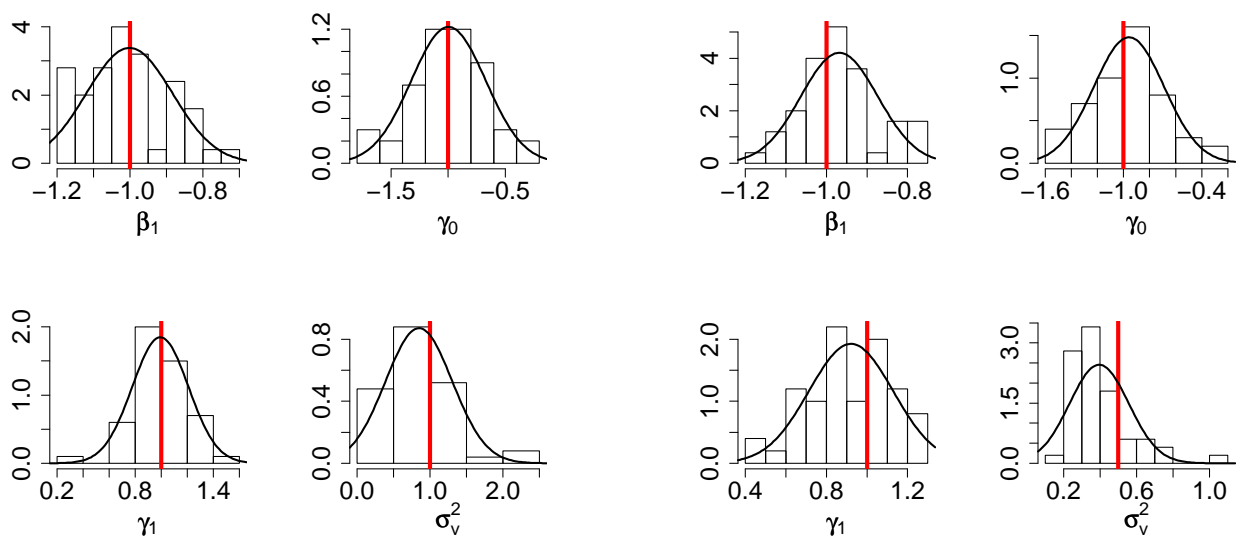

(c) Grupo 11
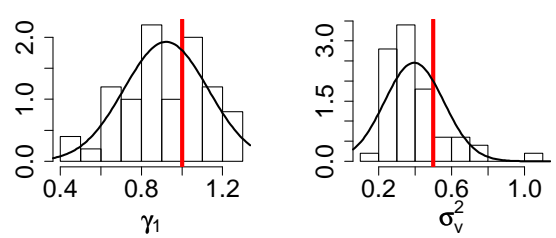

(d) Grupo 12

Figura 6.6: Histogramas das estimativas dos parâmetros estimados via Metropolis-Hastings $-n_{k}=50-$ modelo de mistura - parte 3

Estes histogramas indicam que as estimativas obtidas estão em torno dos valores verdadeiros, representados pelas retas verticais. Indicam também que os valores obtidos não estão muito longe da normalidade assintótica. Note ainda que os valores estimados para $\sigma_{V}^{2}$ estão melhores neste caso em que $n_{k}=50$ do que no caso em que $n_{k}=15$.

Para se avaliar se a convergência do modelo está adequada, calcula-se os valores de $\sqrt{\hat{R}}$, como definido ao final da seção 2.2. Para isso, considere $m=3$ o número de cadeias utilizadas com valores iniciais distintos, em que cada uma apresenta $2 n=30000$ valores, ou seja, $n=15000$. Foram calculados os valores de $\sqrt{\hat{R}}$ para simulações em cada um dos 12 grupos. Os resultados obtidos encontram-se na Tabela 6.4. 
Tabela 6.4: Valores de $\sqrt{\hat{R}}-$ caso $n_{k}=50$ - modelo de mistura

\begin{tabular}{ccccccc}
\hline parâmetro & $\mathbf{1}$ & $\mathbf{2}$ & $\mathbf{3}$ & $\mathbf{4}$ & $\mathbf{5}$ & $\mathbf{6}$ \\
\hline$\beta_{1}$ & 1,00026 & 1,00016 & 1,00078 & 1,00001 & 1,00007 & 1,00023 \\
$\gamma_{0}$ & 1,00038 & 1,00232 & 1,00156 & 1,00030 & 1,00120 & 1,00003 \\
$\gamma_{1}$ & 1,00028 & 1,00144 & 1,00127 & 1,00016 & 1,00035 & 1,00153 \\
$\sigma_{V}^{2}$ & 1,00001 & 1,00025 & 1,00029 & 1,00047 & 1,00021 & 1,00004 \\
\hline \multicolumn{7}{c}{} \\
\hline parâmetro & $\mathbf{7}$ & $\mathbf{8}$ & $\mathbf{9}$ & $\mathbf{1 0}$ & $\mathbf{1 1}$ & $\mathbf{1 2}$ \\
\hline$\beta_{1}$ & 1,00016 & 1,00085 & 1,00035 & 1,00037 & 1,00019 & 1,00107 \\
$\gamma_{0}$ & 1,00062 & 1,00260 & 1,00539 & 1,00253 & 1,00007 & 1,00218 \\
$\gamma_{1}$ & 1,00019 & 1,00024 & 1,00113 & 1,00044 & 1,00027 & 1,00061 \\
$\sigma_{V}^{2}$ & 1,00006 & 1,00045 & 1,00027 & 1,00078 & 1,00046 & 1,00008 \\
\hline
\end{tabular}

É perceptível que todos os valores estão bem próximos a 1 para todas as estimativas de interesse indicando que houve convergência da cadeia e que foi atingida a distribuição de cada estimador.

\subsection{Simulação - abordagem clássica}

Um estudo de simulação da parte clássica foi feito com o auxílio do programa Maple, versão 10. Os dados foram gerados com o programa $\mathrm{R}$ e são os mesmos utilizados na seção 6.2. Os comando utilizados encontram-se no apêndice A.6.

\subsubsection{Caso $n_{k}=15$}

Os resultados obtidos estão na Tabela 6.5, em que a terceira coluna apresenta a média das estimativas obtidas nas 50 simulações. Na quarta coluna está o desvio padrão calculado a partir dos 50 valores de estimativas de cada parâmetro. E, a quinta coluna contém a diferença entre os valores verdadeiros e os médios-estimados. 
Tabela 6.5: Resultado de 50 simulações via REML para $n_{k}=15$ para o modelo de mistura

\begin{tabular}{|c|c|c|c|c|}
\hline \multicolumn{5}{|c|}{ Grupo 1: $\left(\alpha=1,0 ; \sigma_{V}^{2}=0,5 ;\right.$ Censura $\left.=500 ; \beta_{1}=-0,5 ; \gamma_{0}=0 ; \gamma_{1}=0,5 ; \lambda=1\right)$} \\
\hline Parâmetro & Valor verdadeiro & Média da estimativa & DP da estimativa & Verdadeiro - estimado \\
\hline$\beta_{1}$ & $-0,5$ & $-0,4523$ & 0,2011 & $-0,0477$ \\
\hline$\gamma_{0}$ & 0 & $-0,0070$ & 0,3056 & 0,0070 \\
\hline$\gamma_{1}$ & 0,5 & 0,3701 & 0,3607 & 0,1299 \\
\hline$\sigma_{V}^{2}$ & 0,5 & 0,6265 & 0,1435 & $-0,1265$ \\
\hline \multicolumn{5}{|c|}{ Grupo 2: $\left(\alpha=1,3 ; \sigma_{V}^{2}=0,5 ;\right.$ Censura $\left.=500 ; \beta_{1}=-0,5 ; \gamma_{0}=0 ; \gamma_{1}=0,5 ; \lambda=1\right)$} \\
\hline Parâmetro & Valor verdadeiro & Média da estimativa & DP da estimativa & Verdadeiro - estimado \\
\hline$\beta_{1}$ & $-0,5$ & $-0,4863$ & 0,2467 & $-0,0137$ \\
\hline$\gamma_{0}$ & 0 & 0,0459 & 0,2771 & $-0,0459$ \\
\hline$\gamma_{1}$ & 0,5 & 0,6048 & 0,3917 & $-0,1048$ \\
\hline$\sigma_{V}^{2}$ & 0,5 & 0,6129 & 0,1591 & $-0,1129$ \\
\hline \multicolumn{5}{|c|}{ Grupo 3: $\left(\alpha=1 ; \sigma_{V}^{2}=1 ;\right.$ Censura $\left.=500 ; \beta_{1}=-0,5 ; \gamma_{0}=0 ; \gamma_{1}=0,5 ; \lambda=1\right)$} \\
\hline Parâmetro & Valor verdadeiro & Média da estimativa & DP da estimativa & Verdadeiro - estimado \\
\hline$\beta_{1}$ & $-0,5$ & $-0,5075$ & 0,2508 & 0,0075 \\
\hline$\gamma_{0}$ & 0 & 0,0102 & 0,3300 & $-0,0102$ \\
\hline$\gamma_{1}$ & 0,5 & 0,5005 & 0,3805 & $-0,0005$ \\
\hline$\sigma_{V}^{2}$ & 1 & 0,7787 & 0,2061 & 0,2213 \\
\hline \multicolumn{5}{|c|}{ Grupo 4: $\left(\alpha=1 ; \sigma_{V}^{2}=0,5 ;\right.$ Censura $\left.=300 ; \beta_{1}=-0,5 ; \gamma_{0}=0 ; \gamma_{1}=0,5 ; \lambda=1\right)$} \\
\hline Parâmetro & Valor verdadeiro & Média da estimativa & DP da estimativa & Verdadeiro - estimado \\
\hline$\beta_{1}$ & $-0,5$ & $-0,4576$ & 0,2308 & $-0,0424$ \\
\hline$\gamma_{0}$ & 0 & $-0,0066$ & 0,2745 & 0,0066 \\
\hline$\gamma_{1}$ & 0,5 & 0,4977 & 0,3646 & 0,0023 \\
\hline$\sigma_{V}^{2}$ & 0,5 & 0,6609 & 0,1812 & $-0,1609$ \\
\hline \multicolumn{5}{|c|}{ Grupo $5:\left(\alpha=1 ; \sigma_{V}^{2}=0,5 ;\right.$ Censura $\left.=500 ; \beta_{1}=-0,5 ; \gamma_{0}=-0,5 ; \gamma_{1}=0,5 ; \lambda=1\right)$} \\
\hline Parâmetro & Valor verdadeiro & Média da estimativa & DP da estimativa & Verdadeiro - estimado \\
\hline$\beta_{1}$ & $-0,5$ & $-0,4987$ & 0,1912 & $-0,0013$ \\
\hline$\gamma_{0}$ & $-0,5$ & $-0,4590$ & 0,3081 & $-0,0410$ \\
\hline$\gamma_{1}$ & 0,5 & 0,5152 & 0,3227 & $-0,0152$ \\
\hline$\sigma_{V}^{2}$ & 0,5 & 0,6351 & 0,1542 & $-0,1351$ \\
\hline \multicolumn{5}{|c|}{ Grupo $6:\left(\alpha=1,3 ; \sigma_{V}^{2}=0,5 ;\right.$ Censura $\left.=500 ; \beta_{1}=-0,5 ; \gamma_{0}=-0,5 ; \gamma_{1}=0,5 ; \lambda=1\right)$} \\
\hline Parâmetro & Valor verdadeiro & Média da estimativa & DP da estimativa & Verdadeiro - estimado \\
\hline$\beta_{1}$ & $-0,5$ & $-0,4651$ & 0,1979 & $-0,0349$ \\
\hline$\gamma_{0}$ & $-0,5$ & $-0,4299$ & 0,3402 & $-0,0701$ \\
\hline$\gamma_{1}$ & 0,5 & 0,4771 & 0,3833 & 0,0229 \\
\hline$\sigma_{V}^{2}$ & 0,5 & 0,5741 & 0,1338 & $-0,0741$ \\
\hline
\end{tabular}




\begin{tabular}{|c|c|c|c|c|}
\hline \multicolumn{5}{|c|}{ Grupo $7:\left(\alpha=1 ; \sigma_{V}^{2}=1 ;\right.$ Censura $\left.=500 ; \beta_{1}=-0,5 ; \gamma_{0}=-0,5 ; \gamma_{1}=0,5 ; \lambda=1\right)$} \\
\hline Parâmetro & Valor verdadeiro & Média da estimativa & DP da estimativa & Verdadeiro - estimado \\
\hline$\beta_{1}$ & $-0,5$ & $-0,4385$ & 0,2023 & $-0,0615$ \\
\hline$\gamma_{0}$ & $-0,5$ & $-0,3993$ & 0,3304 & $-0,1007$ \\
\hline$\gamma_{1}$ & 0,5 & 0,3652 & 0,4038 & 0,1348 \\
\hline$\sigma_{V}^{2}$ & 1 & 0,7950 & 0,1807 & 0,2050 \\
\hline \multicolumn{5}{|c|}{ Grupo 8: $\left(\alpha=1 ; \sigma_{V}^{2}=0,5 ;\right.$ Censura $\left.=300 ; \beta_{1}=-0,5 ; \gamma_{0}=-0,5 ; \gamma_{1}=0,5 ; \lambda=1\right)$} \\
\hline Parâmetro & Valor verdadeiro & Média da estimativa & DP da estimativa & Verdadeiro - estimado \\
\hline$\beta_{1}$ & $-0,5$ & $-0,4661$ & 0,1828 & $-0,0339$ \\
\hline$\gamma_{0}$ & $-0,5$ & $-0,4828$ & 0,2420 & $-0,0172$ \\
\hline$\gamma_{1}$ & 0,5 & 0,4360 & 0,2923 & 0,0640 \\
\hline$\sigma_{V}^{2}$ & 0,5 & 0,6414 & 0,1858 & $-0,1414$ \\
\hline \multicolumn{5}{|c|}{ Grupo 9: $\left(\alpha=1 ; \sigma_{V}^{2}=0,5 ;\right.$ Censura $\left.=500 ; \beta_{1}=-1 ; \gamma_{0}=-1 ; \gamma_{1}=1 ; \lambda=1\right)$} \\
\hline Parâmetro & Valor verdadeiro & Média da estimativa & DP da estimativa & Verdadeiro - estimado \\
\hline$\beta_{1}$ & -1 & $-0,9991$ & 0,2166 & $-0,0009$ \\
\hline$\gamma_{0}$ & -1 & $-0,8801$ & 0,3234 & $-0,1199$ \\
\hline$\gamma_{1}$ & 1 & 0,9175 & 0,3163 & 0,0825 \\
\hline$\sigma_{V}^{2}$ & 0,5 & 0,6741 & 0,1818 & $-0,1741$ \\
\hline \multicolumn{5}{|c|}{ Grupo 10: $\left(\alpha=1,3 ; \sigma_{V}^{2}=0,5 ;\right.$ Censura $\left.=500 ; \beta_{1}=-1 ; \gamma_{0}=-1 ; \gamma_{1}=1 ; \lambda=1\right)$} \\
\hline Parâmetro & Valor verdadeiro & Média da estimativa & DP da estimativa & Verdadeiro - estimado \\
\hline$\beta_{1}$ & -1 & $-0,9434$ & 0,2167 & $-0,0566$ \\
\hline$\gamma_{0}$ & -1 & $-0,9822$ & 0,3285 & $-0,0178$ \\
\hline$\gamma_{1}$ & 1 & 0,9606 & 0,4443 & 0,0394 \\
\hline$\sigma_{V}^{2}$ & 0,5 & 0,6097 & 0,1347 & $-0,1097$ \\
\hline \multicolumn{5}{|c|}{ Grupo 11: $\left(\alpha=1 ; \sigma_{V}^{2}=1 ;\right.$ Censura $\left.=500 ; \beta_{1}=-1 ; \gamma_{0}=-1 ; \gamma_{1}=1 ; \lambda=1\right)$} \\
\hline Parâmetro & Valor verdadeiro & Média da estimativa & DP da estimativa & Verdadeiro - estimado \\
\hline$\beta_{1}$ & -1 & $-0,9670$ & 0,2316 & $-0,0330$ \\
\hline$\gamma_{0}$ & -1 & $-0,9280$ & 0,3767 & $-0,0720$ \\
\hline$\gamma_{1}$ & 1 & 0,9287 & 0,3252 & 0,0713 \\
\hline$\sigma_{V}^{2}$ & 1 & 0,8357 & 0,2166 & 0,1643 \\
\hline \multicolumn{5}{|c|}{ Grupo 12: $\left(\alpha=1 ; \sigma_{V}^{2}=0,5 ;\right.$ Censura $\left.=300 ; \beta_{1}=-1 ; \gamma_{0}=-1 ; \gamma_{1}=1 ; \lambda=1\right)$} \\
\hline Parâmetro & Valor verdadeiro & Média da estimativa & DP da estimativa & Verdadeiro - estimado \\
\hline$\beta_{1}$ & -1 & $-0,9780$ & 0,2071 & $-0,0220$ \\
\hline$\gamma_{0}$ & -1 & $-0,9679$ & 0,3127 & $-0,0321$ \\
\hline$\gamma_{1}$ & 1 & 1,0251 & 0,4179 & $-0,0251$ \\
\hline$\sigma_{V}^{2}$ & 0,5 & 0,6081 & 0,1480 & $-0,1081$ \\
\hline
\end{tabular}

Note que, a diferença entre os valores médios estimados e os valores verdadeiros são relativamente pequenas, o que indica que os resultados obtidos via simulação estão bons. 
As Figuras de 6.7 a 6.9 mostram os histogramas feitos a partir das estimativas calculadas nas 50 simulações, para cada um dos parâmetros.
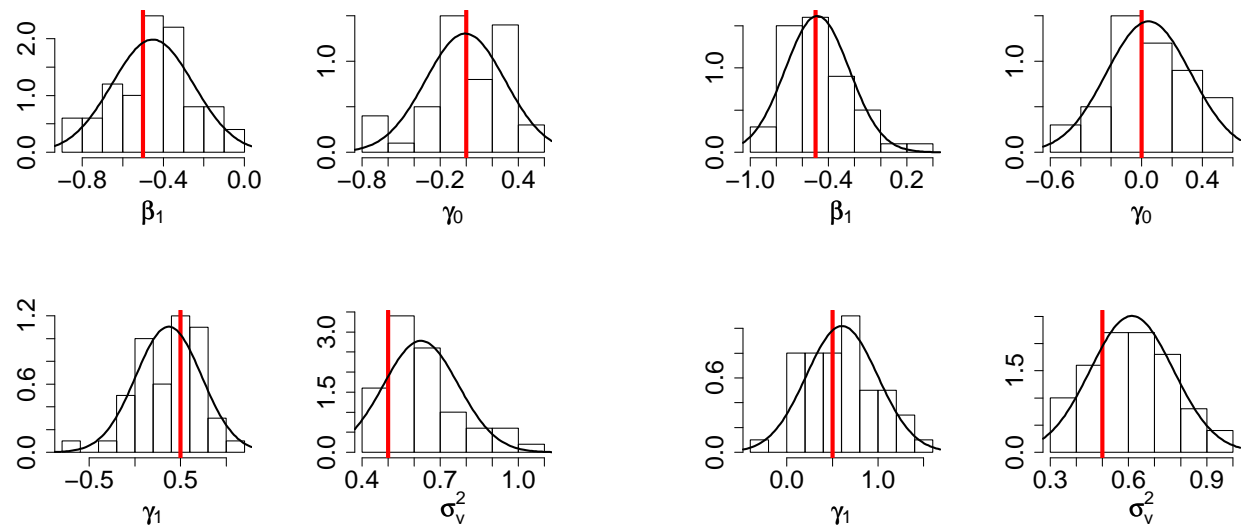

(a) Grupo 1

(b) Grupo 2
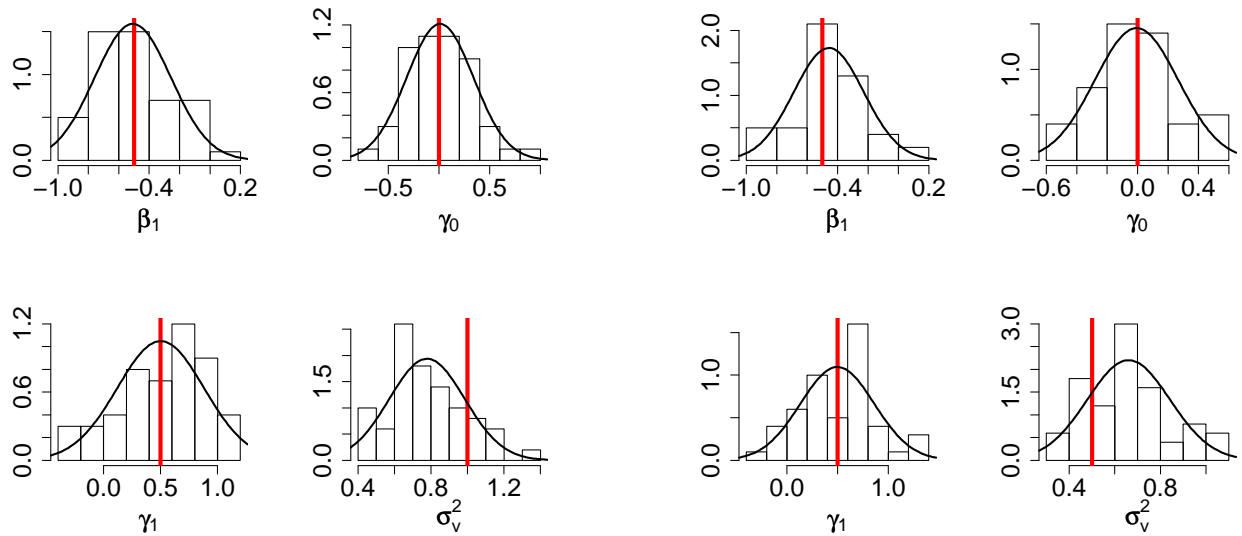

(c) Grupo 3

(d) Grupo 4

Figura 6.7: Histogramas das estimativas dos parâmetros estimados via REML $-n_{k}=15$ - modelo de mistura - parte 1 

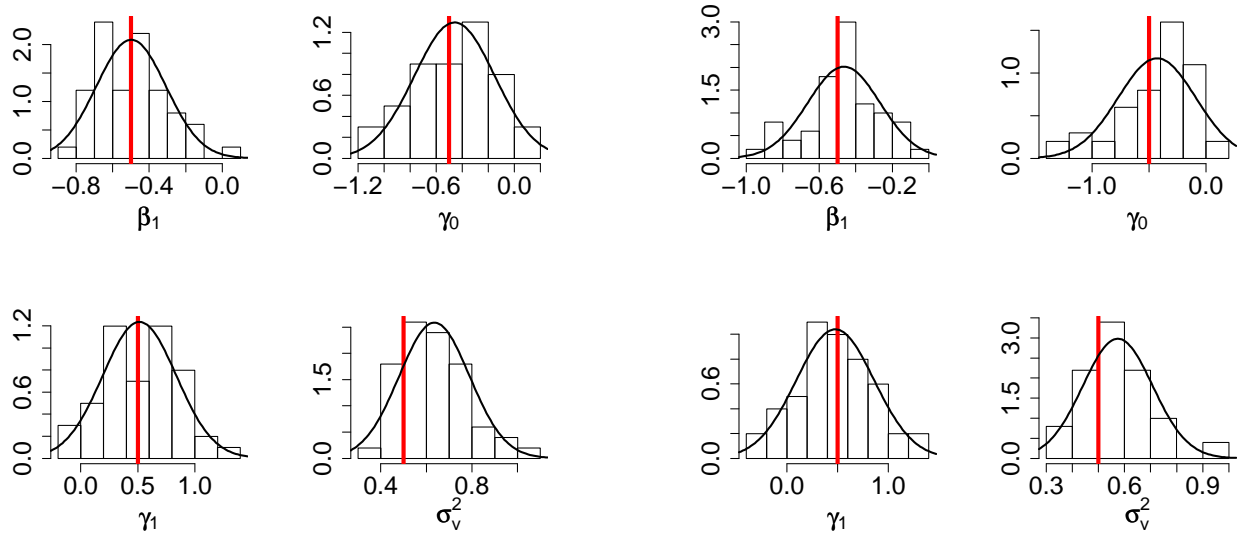

(a) Grupo 5

(b) Grupo 6
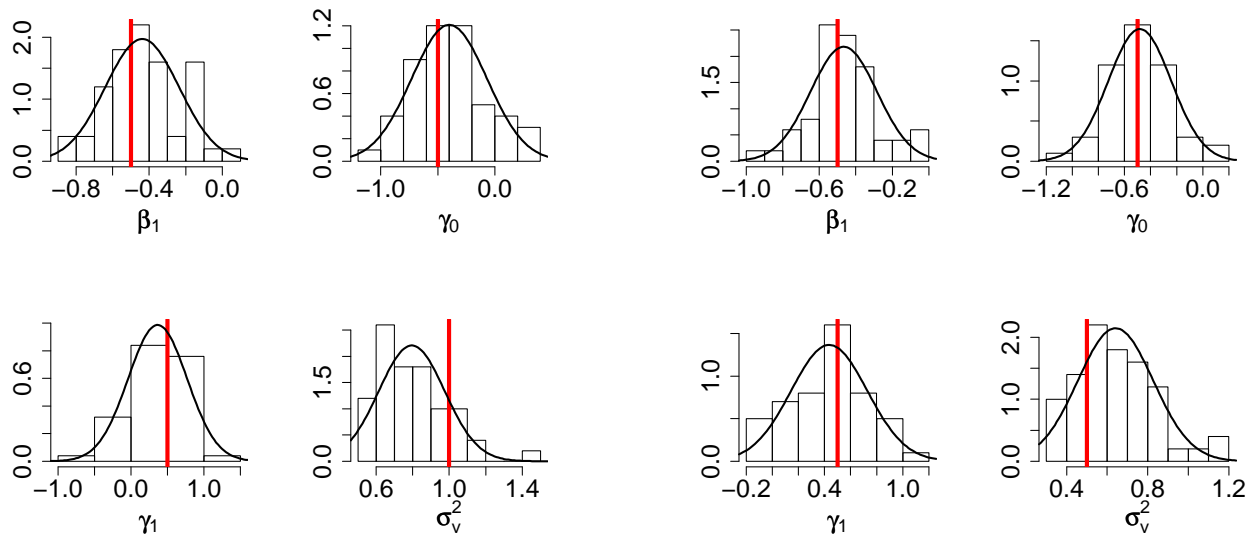

(c) Grupo 7

(d) Grupo 8

Figura 6.8: Histogramas das estimativas dos parâmetros estimados via REML $-n_{k}=15$ - modelo de mistura - parte 2 

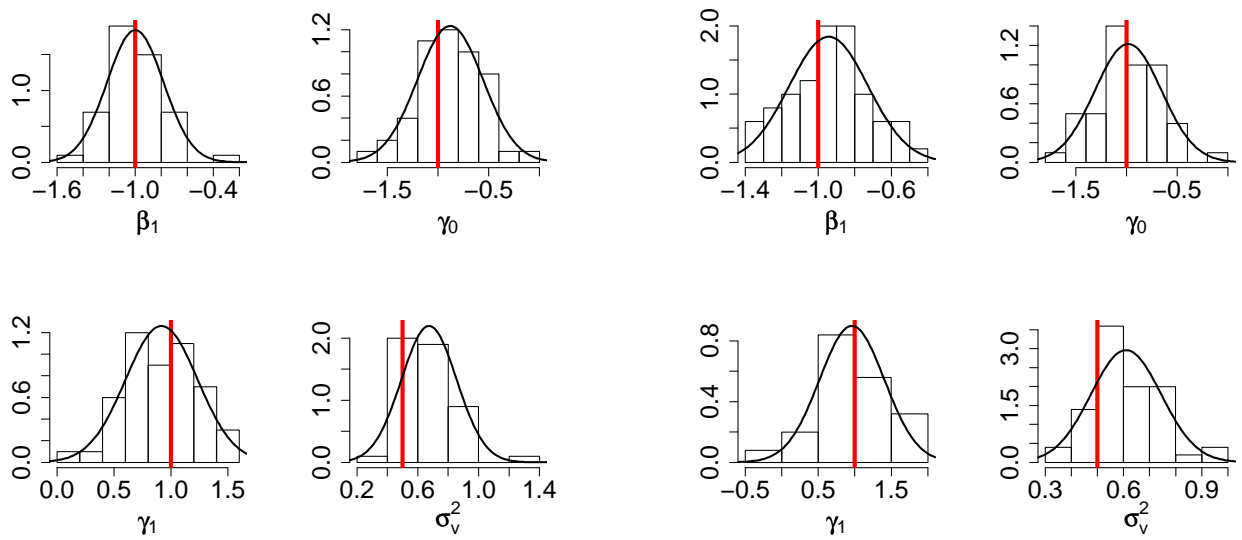

(a) Grupo 9

(b) Grupo 10
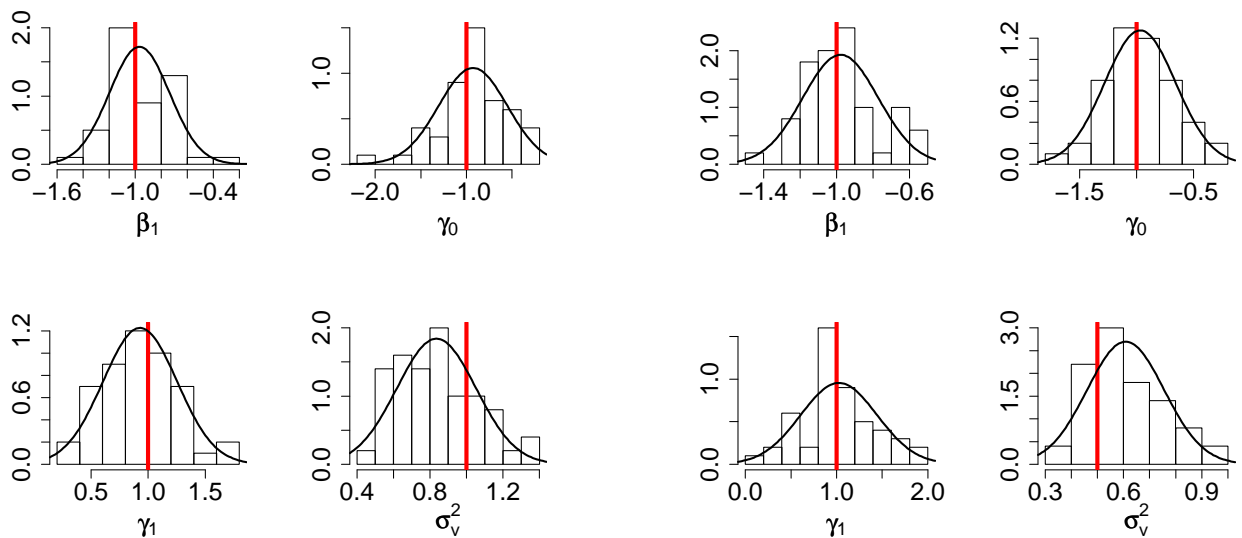

(c) Grupo 11
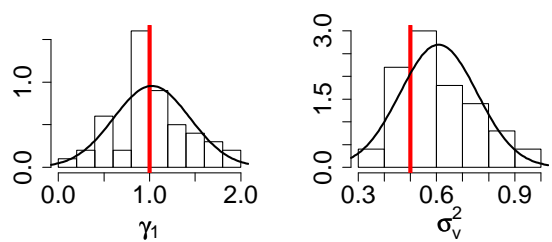

(d) Grupo 12

Figura 6.9: Histogramas das estimativas dos parâmetros estimados via REML $-n_{k}=15$ - modelo de mistura - parte 3

Estes histogramas indicam que as estimativas obtidas estão em torno dos valores verdadeiros, representados pelas retas verticais. Indicam também que os valores obtidos não estão muito longe da normalidade assintótica.

\subsubsection{Caso $n_{k}=50$}

Os resultados obtidos estão na Tabela 6.6, em que a terceira coluna apresenta a média das estimativas obtidas nas 50 simulações. Na quarta coluna está o desvio padrão calculado a partir dos 50 valores de estimativas de cada parâmetro. E, a quinta coluna contém a diferença entre os valores verdadeiros e os médios-estimados. 
Tabela 6.6: Resultado de 50 simulações via REML para $n_{k}=50$ para o modelo de mistura

\begin{tabular}{|c|c|c|c|c|}
\hline \multicolumn{5}{|c|}{ Grupo 1: $\left(\alpha=1,0 ; \sigma_{V}^{2}=0,5 ;\right.$ Censura $\left.=500 ; \beta_{1}=-0,5 ; \gamma_{0}=0 ; \gamma_{1}=0,5 ; \lambda=1\right)$} \\
\hline Parâmetro & Valor verdadeiro & Média da estimativa & DP da estimativa & Verdadeiro - estimado \\
\hline$\beta_{1}$ & $-0,5$ & $-0,4728$ & 0,1135 & $-0,0272$ \\
\hline$\gamma_{0}$ & 0 & 0,0060 & 0,2381 & $-0,0060$ \\
\hline$\gamma_{1}$ & 0,5 & 0,4931 & 0,1923 & 0,0069 \\
\hline$\sigma_{V}^{2}$ & 0,5 & 0,5100 & 0,1360 & $-0,0100$ \\
\hline \multicolumn{5}{|c|}{ Grupo 2: $\left(\alpha=1,3 ; \sigma_{V}^{2}=0,5 ;\right.$ Censura $\left.=500 ; \beta_{1}=-0,5 ; \gamma_{0}=0 ; \gamma_{1}=0,5 ; \lambda=1\right)$} \\
\hline Parâmetro & Valor verdadeiro & Média da estimativa & DP da estimativa & Verdadeiro - estimado \\
\hline$\beta_{1}$ & $-0,5$ & $-0,4927$ & 0,1368 & $-0,0073$ \\
\hline$\gamma_{0}$ & 0 & 0,0713 & 0,2215 & $-0,0713$ \\
\hline$\gamma_{1}$ & 0,5 & 0,4918 & 0,1933 & 0,0082 \\
\hline$\sigma_{V}^{2}$ & 0,5 & 0,5338 & 0,2160 & $-0,0338$ \\
\hline \multicolumn{5}{|c|}{ Grupo 3: $\left(\alpha=1 ; \sigma_{V}^{2}=1 ;\right.$ Censura $\left.=500 ; \beta_{1}=-0,5 ; \gamma_{0}=0 ; \gamma_{1}=0,5 ; \lambda=1\right)$} \\
\hline Parâmetro & Valor verdadeiro & Média da estimativa & DP da estimativa & Verdadeiro - estimado \\
\hline$\beta_{1}$ & $-0,5$ & $-0,4560$ & 0,1133 & $-0,0440$ \\
\hline$\gamma_{0}$ & 0 & $-0,0264$ & 0,3328 & 0,0264 \\
\hline$\gamma_{1}$ & 0,5 & 0,4932 & 0,1836 & 0,0068 \\
\hline$\sigma_{V}^{2}$ & 1 & 0,8095 & 0,2842 & 0,1905 \\
\hline \multicolumn{5}{|c|}{ Grupo 4: $\left(\alpha=1 ; \sigma_{V}^{2}=0,5 ;\right.$ Censura $\left.=300 ; \beta_{1}=-0,5 ; \gamma_{0}=0 ; \gamma_{1}=0,5 ; \lambda=1\right)$} \\
\hline Parâmetro & Valor verdadeiro & Média da estimativa & DP da estimativa & Verdadeiro - estimado \\
\hline$\beta_{1}$ & $-0,5$ & $-0,4667$ & 0,1312 & $-0,0333$ \\
\hline$\gamma_{0}$ & 0 & $-0,0069$ & 0,2528 & 0,0069 \\
\hline$\gamma_{1}$ & 0,5 & 0,5060 & 0,1607 & $-0,0060$ \\
\hline$\sigma_{V}^{2}$ & 0,5 & 0,5569 & 0,1603 & $-0,0569$ \\
\hline \multicolumn{5}{|c|}{ Grupo 5: $\left(\alpha=1 ; \sigma_{V}^{2}=0,5 ;\right.$ Censura $\left.=500 ; \beta_{1}=-0,5 ; \gamma_{0}=-0,5 ; \gamma_{1}=0,5 ; \lambda=1\right)$} \\
\hline Parâmetro & Valor verdadeiro & Média da estimativa & DP da estimativa & Verdadeiro - estimado \\
\hline$\beta_{1}$ & $-0,5$ & $-0,4663$ & 0,1049 & $-0,0337$ \\
\hline$\gamma_{0}$ & $-0,5$ & $-0,4360$ & 0,2580 & $-0,0640$ \\
\hline$\gamma_{1}$ & 0,5 & 0,4624 & 0,1889 & 0,0376 \\
\hline$\sigma_{V}^{2}$ & 0,5 & 0,5212 & 0,1569 & $-0,0212$ \\
\hline
\end{tabular}




\begin{tabular}{|c|c|c|c|c|}
\hline \multicolumn{5}{|c|}{ Grupo 6: $\left(\alpha=1,3 ; \sigma_{V}^{2}=0,5 ;\right.$ Censura $\left.=500 ; \beta_{1}=-0,5 ; \gamma_{0}=-0,5 ; \gamma_{1}=0,5 ; \lambda=1\right)$} \\
\hline Parâmetro & Valor verdadeiro & Média da estimativa & DP da estimativa & Verdadeiro - estimado \\
\hline$\beta_{1}$ & $-0,5$ & $-0,4712$ & 0,1119 & $-0,0288$ \\
\hline$\gamma_{0}$ & $-0,5$ & $-0,4638$ & 0,2328 & $-0,0362$ \\
\hline$\gamma_{1}$ & 0,5 & 0,4972 & 0,1973 & 0,0028 \\
\hline$\sigma_{V}^{2}$ & 0,5 & 0,5562 & 0,1985 & $-0,0562$ \\
\hline \multicolumn{5}{|c|}{ Grupo 7: $\left(\alpha=1 ; \sigma_{V}^{2}=1 ;\right.$ Censura $\left.=500 ; \beta_{1}=-0,5 ; \gamma_{0}=-0,5 ; \gamma_{1}=0,5 ; \lambda=1\right)$} \\
\hline Parâmetro & Valor verdadeiro & Média da estimativa & DP da estimativa & Verdadeiro - estimado \\
\hline$\beta_{1}$ & $-0,5$ & $-0,4724$ & 0,1068 & $-0,0276$ \\
\hline$\gamma_{0}$ & $-0,5$ & $-0,4281$ & 0,2867 & $-0,0719$ \\
\hline$\gamma_{1}$ & 0,5 & 0,5256 & 0,1957 & $-0,0256$ \\
\hline$\sigma_{V}^{2}$ & 1 & 0,8070 & 0,3210 & 0,1930 \\
\hline \multicolumn{5}{|c|}{ Grupo 8: $\left(\alpha=1 ; \sigma_{V}^{2}=0,5 ;\right.$ Censura $\left.=300 ; \beta_{1}=-0,5 ; \gamma_{0}=-0,5 ; \gamma_{1}=0,5 ; \lambda=1\right)$} \\
\hline Parâmetro & Valor verdadeiro & Média da estimativa & DP da estimativa & Verdadeiro - estimado \\
\hline$\beta_{1}$ & $-0,5$ & $-0,4487$ & 0,1063 & $-0,0513$ \\
\hline$\gamma_{0}$ & $-0,5$ & $-0,5283$ & 0,2304 & 0,0283 \\
\hline$\gamma_{1}$ & 0,5 & 0,4868 & 0,1968 & 0,0132 \\
\hline$\sigma_{V}^{2}$ & 0,5 & 0,5450 & 0,1728 & $-0,0450$ \\
\hline \multicolumn{5}{|c|}{ Grupo 9: $\left(\alpha=1 ; \sigma_{V}^{2}=0,5 ;\right.$ Censura $\left.=500 ; \beta_{1}=-1 ; \gamma_{0}=-1 ; \gamma_{1}=1 ; \lambda=1\right)$} \\
\hline Parâmetro & Valor verdadeiro & Média da estimativa & DP da estimativa & Verdadeiro - estimado \\
\hline$\beta_{1}$ & -1 & $-0,9767$ & 0,1186 & $-0,0233$ \\
\hline$\gamma_{0}$ & -1 & $-0,9859$ & 0,2582 & $-0,0141$ \\
\hline$\gamma_{1}$ & 1 & 1,0294 & 0,2051 & $-0,0294$ \\
\hline$\sigma_{V}^{2}$ & 0,5 & 0,5422 & 0,1555 & $-0,0422$ \\
\hline \multicolumn{5}{|c|}{ Grupo 10: $\left(\alpha=1,3 ; \sigma_{V}^{2}=0,5 ;\right.$ Censura $\left.=500 ; \beta_{1}=-1 ; \gamma_{0}=-1 ; \gamma_{1}=1 ; \lambda=1\right)$} \\
\hline Parâmetro & Valor verdadeiro & Média da estimativa & DP da estimativa & Verdadeiro - estimado \\
\hline$\beta_{1}$ & -1 & $-0,9775$ & 0,1191 & $-0,0225$ \\
\hline$\gamma_{0}$ & -1 & $-0,9732$ & 0,2593 & $-0,0268$ \\
\hline$\gamma_{1}$ & 1 & 0,9803 & 0,2235 & 0,0197 \\
\hline$\sigma_{V}^{2}$ & 0,5 & 0,5694 & 0,1981 & $-0,0694$ \\
\hline \multicolumn{5}{|c|}{ Grupo 11: $\left(\alpha=1 ; \sigma_{V}^{2}=1 ;\right.$ Censura $\left.=500 ; \beta_{1}=-1 ; \gamma_{0}=-1 ; \gamma_{1}=1 ; \lambda=1\right)$} \\
\hline Parâmetro & Valor verdadeiro & Média da estimativa & DP da estimativa & Verdadeiro - estimado \\
\hline$\beta_{1}$ & -1 & $-0,9912$ & 0,1143 & $-0,0088$ \\
\hline$\gamma_{0}$ & -1 & $-1,0061$ & 0,3266 & 0,0061 \\
\hline$\gamma_{1}$ & 1 & 1,0072 & 0,2164 & $-0,0072$ \\
\hline$\sigma_{V}^{2}$ & 1 & 0,9018 & 0,3301 & 0,0982 \\
\hline \multicolumn{5}{|c|}{ Grupo 12: $\left(\alpha=1 ; \sigma_{V}^{2}=0,5 ;\right.$ Censura $\left.=300 ; \beta_{1}=-1 ; \gamma_{0}=-1 ; \gamma_{1}=1 ; \lambda=1\right)$} \\
\hline Parâmetro & Valor verdadeiro & Média da estimativa & DP da estimativa & Verdadeiro - estimado \\
\hline$\beta_{1}$ & -1 & $-0,9572$ & 0,0914 & $-0,0428$ \\
\hline$\gamma_{0}$ & -1 & $-0,9737$ & 0,2682 & $-0,0263$ \\
\hline$\gamma_{1}$ & 1 & 0,9450 & 0,2034 & 0,0550 \\
\hline$\sigma_{V}^{2}$ & 0,5 & 0,5374 & 0,1631 & $-0,0374$ \\
\hline
\end{tabular}

Note que, a diferença entre os valores médios estimados e os valores verdadeiros são menores do que um desvio-padrão, o que indica que os resultados obtidos via simulação estão precisos. Note também que esta diferença é menor do que no caso $n_{k}=15$. Ou seja, como no caso bayesiano, o fato de termos mais indivíduos em cada clínica nos fornece valores estimados mais acurados. 
As Figuras de 6.10 a 6.12 mostram os histogramas feitos a partir das estimativas calculadas nas 50 simulações, para cada um dos parâmetros.
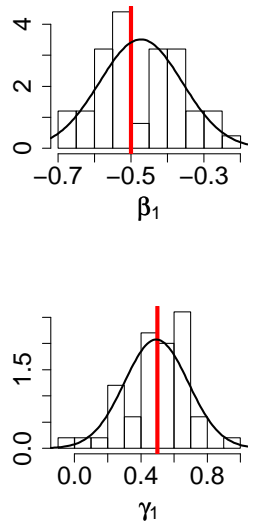

(a) Grupo 1
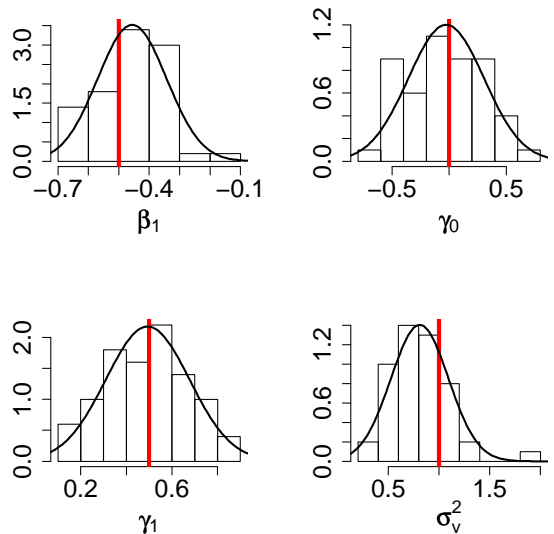

(c) Grupo 3
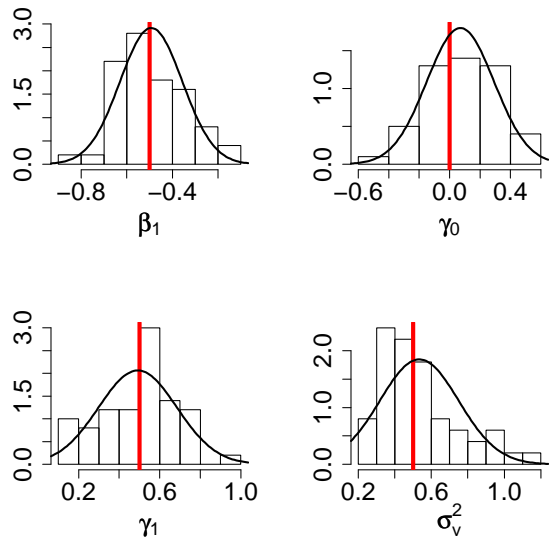

(b) Grupo 2
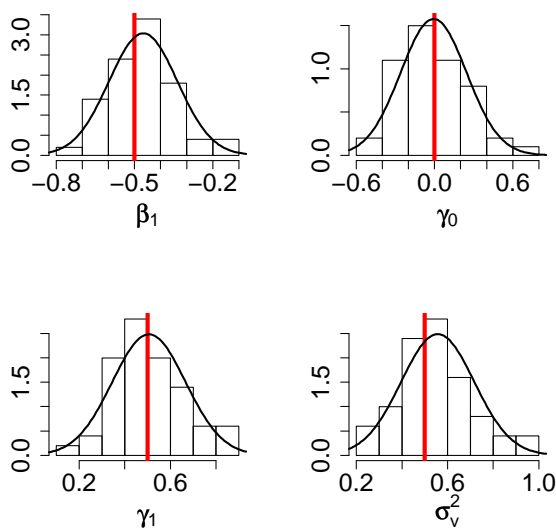

(d) Grupo 4

Figura 6.10: Histogramas das estimativas dos parâmetros estimados via REML $-n_{k}=50$ - modelo de mistura - parte 1 

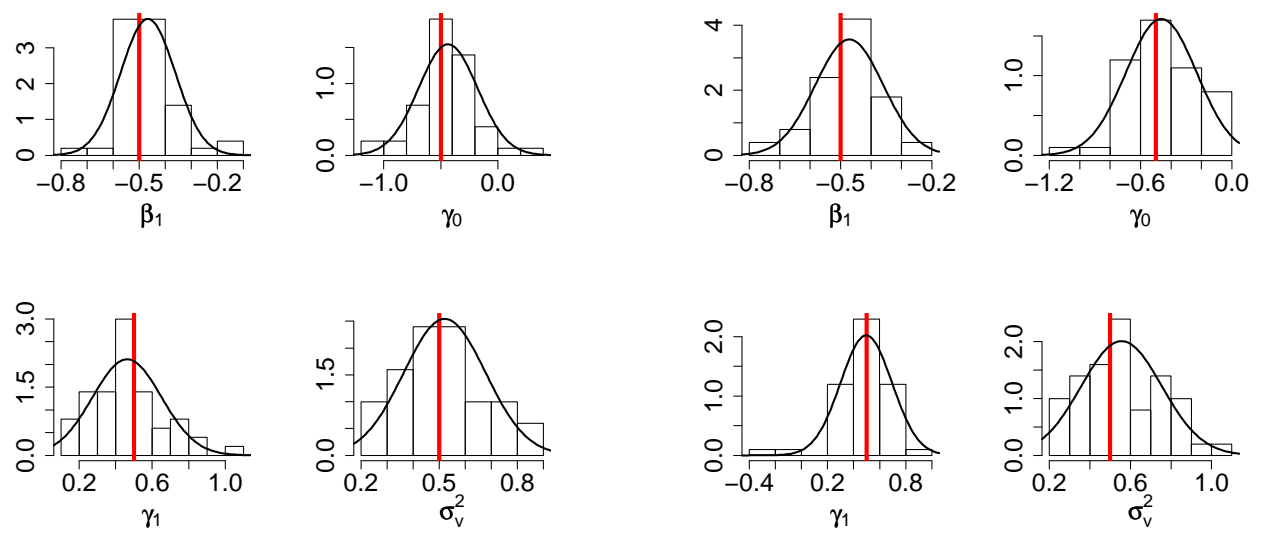

(a) Grupo 5

(b) Grupo 6
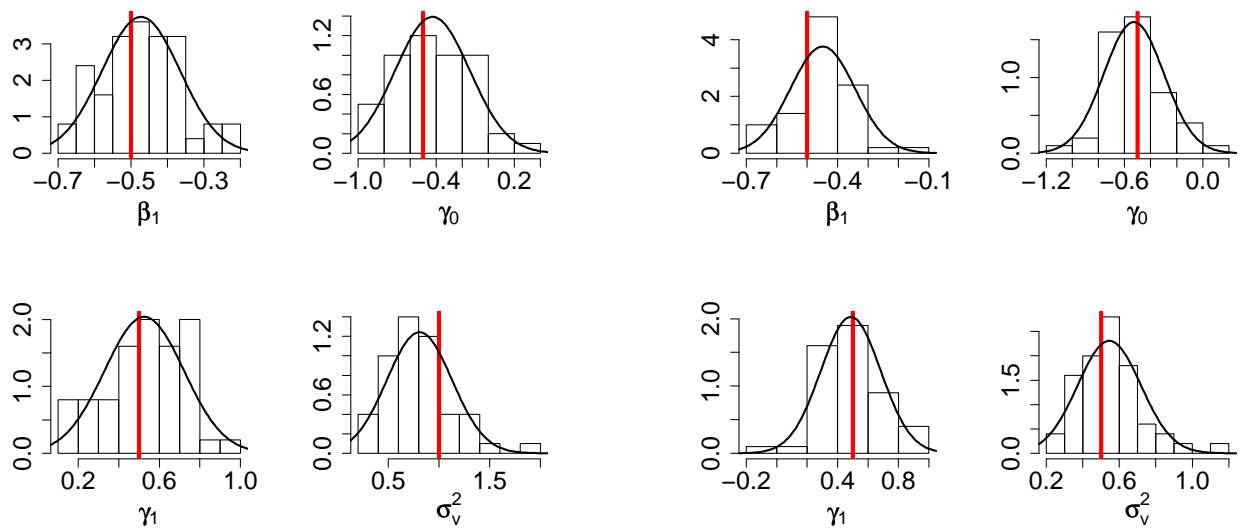

(c) Grupo 7

(d) Grupo 8

Figura 6.11: Histogramas das estimativas dos parâmetros estimados via REML $-n_{k}=50$ - modelo de mistura - parte 2 

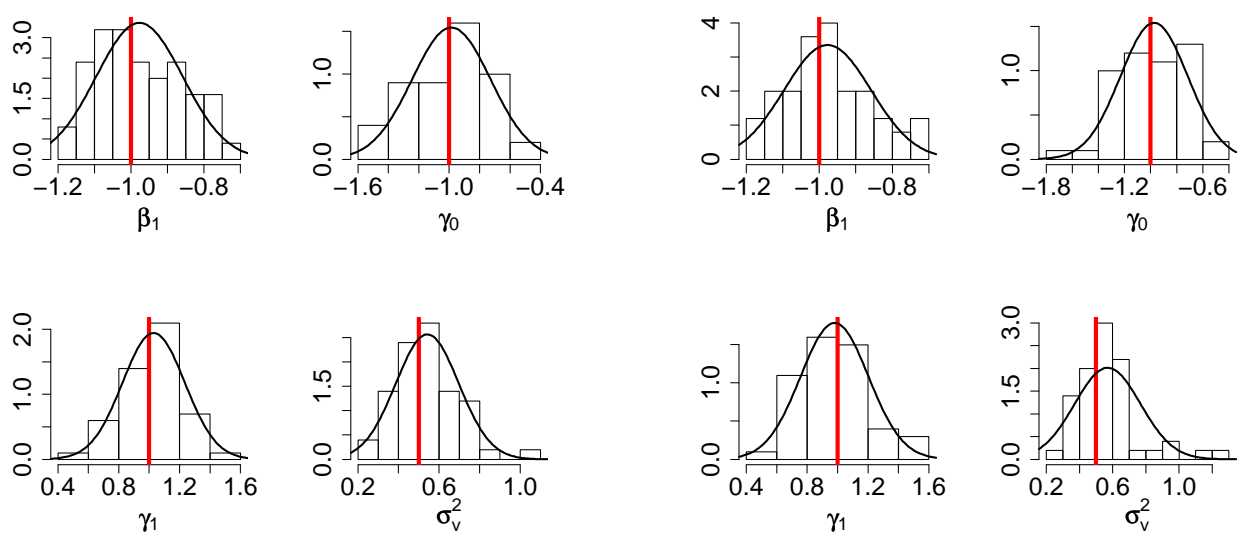

(a) Grupo 9

(b) Grupo 10
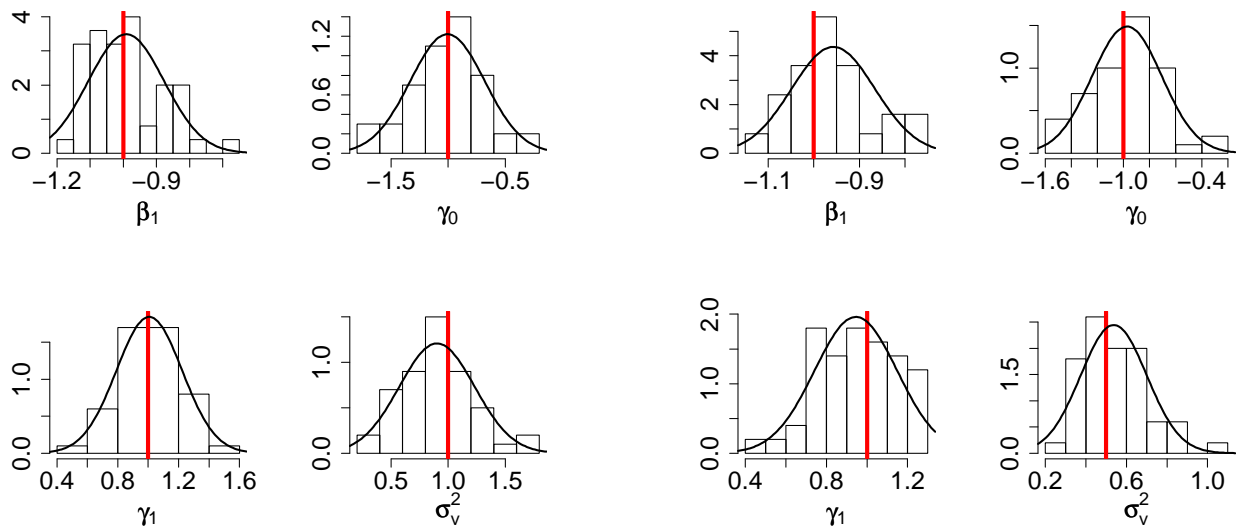

(c) Grupo 11
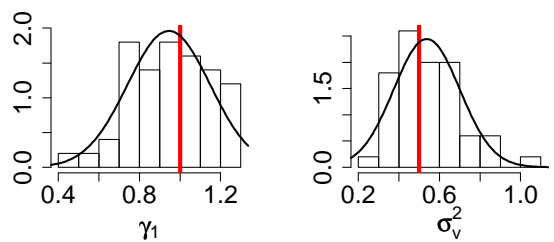

(d) Grupo 12

Figura 6.12: Histogramas das estimativas dos parâmetros estimados via REML $-n_{k}=50$ - modelo de mistura - parte 3

Estes histogramas indicam que as estimativas obtidas estão em torno dos valores verdadeiros, representados pelas retas verticais. Indicam também que os valores obtidos não estão muito longe da normalidade assintótica. Este resultado é semelhante a todos os casos estudados.

\subsection{Conclusões}

Os resultados, tanto bayesiano quanto clássico, mostram que os valores obtidos nas estimativas estão próximos aos valores reais, o que sugere que as estimações utilizando ambos os métodos de inferência implementados foram adequadas. A única ressalva se deve quanto à estimação de $\sigma_{V}^{2}$ via Metropolis-Hastings, em que no caso $n_{k}=15$, há uma subestimação e as estimativas não estão muito próximas dos valores verdadeiros.

Para $\beta_{1}, \gamma_{0}$ e $\gamma_{1}$, praticamente em todos os casos, a diferença entre a média do valor estimado e o 
valor verdadeiro é menor do que um desvio-padrão, o que sugere que os resultados estão razoavelmente precisos. Os histogramas feitos com os valores estimados nas simulações ilustram que os valores verdadeiros, representados pelas retas verticais, estão dentro do esperado. E, que os valores têm distribuições aproximadamente normais.

Com relação à convergência das cadeias, na abordagem bayesiana, os valores de $\sqrt{\hat{R}}$ estão bem próximos a 1 para todas as estimativas de interesse indicando que houve a convergência das cadeias e que foi atingida a distribuição de cada estimador.

Nas simulações clássica e bayesiana, os valores estimados no caso $n_{k}=50$ estão mais próximos dos valores verdadeiros do que no caso $n_{k}=15$, mas em ambos os casos os valores estimados e verdadeiros diferem, quase sempre, por uma quantidade menor que um desvio-padrão. Note que, para $\sigma_{V}^{2}$, com abordagem bayesiana, os resultados obtidos com $n_{k}=50$ estão bem melhores do que os com $n_{k}=15$.

Note, também, que, em ambas as abordagens, a diferença entre a média das estimativas e o valor verdadeiro para o caso $n_{k}=50$ é menor do que no caso $n_{k}=15$. Ou seja, o fato de termos mais indivíduos em cada clínica nos fornece valores estimados mais acurados.

Com relação aos desvios-padrão das estimativas, é perceptível que os valores estão, quase sempre, menores no caso $n_{k}=50$ em comparação com $n_{k}=15$. A exceção ocorre no caso de $\sigma_{v}^{2}$ em que essa situação se inverte, mas a acurácia do valor estimado é melhor em $n_{k}=50$ do que em $n_{k}=15$. Isso em ambas as inferências, bayesiana e clássica. 
86CAPÍTULO 6. ESTUDOS DE SIMULAÇÃO - MODELO DE MISTURA COM EFEITOS ALEATÓRIOS 


\section{Capítulo 7}

\section{Análise de dados - modelo de mistura com efeitos aleatórios}

Para ilustrar uma aplicação de uso do modelo de mistura com efeitos aleatórios nas frações de curados e não curados, Yau e Ng (2001) utilizaram um conjunto de dados em Kalbfleisch e Prentice (1980, pp.225-229). Tais dados são parte de um estudo feito pelo Radiation Therapy Oncology Group nos Estados Unidos.

A seguir, é apresentada a utilização deste mesmo conjunto de dados no modelo apresentado no Capítulo 5, para fração de cura com efeitos aleatórios na fração de curados, a partir do modelo de mistura. Os dados foram descritos na seção 4.1.

\subsection{Inferência bayesiana via Metropolis-Hastings}

Uma primeira análise desses dados foi feita com a utilização do programa Winbugs (versão 1.4). Os comandos utilizados encontram-se no apêndice A.5. Os resultados obtidos estão apresentados na Tabela 7.1, em que a coluna de média apresenta o valor da média entre os valores amostrados, DP apresenta o desvio-padrão e erro MC está mostrando os valores obtidos pelo erro padrão de Monte Carlo para a média.

Tabela 7.1: Resultados - câncer na orofaringe - modelo de mistura com efeitos aleatórios Metropolis-Hastings

\begin{tabular}{ccccccccc}
\hline \multicolumn{7}{c}{ Resultados das posterioris, baseados em 30000 amostras } \\
\hline parâmetro & média & DP & erro MC & $\mathbf{2 , 5 0 \%}$ & mediana & $\mathbf{9 7 , 5 0 \%}$ & início & amostras \\
\hline$\alpha$ & 0,67740 & 0,03343 & 0,00029 & 0,61040 & 0,67790 & 0,74150 & 4001 & 78000 \\
$\beta_{1}$ & $-0,29330$ & 0,27830 & 0,00249 & $-0,86610$ & $-0,28540$ & 0,22860 & 4001 & 78000 \\
$\gamma_{0}$ & $-5,63400$ & 1,60300 & 0,01496 & $-9,27800$ & $-5,42700$ & $-3,13000$ & 4001 & 78000 \\
$\gamma_{1}$ & $-1,43000$ & 2,52100 & 0,02185 & $-6,73700$ & $-1,26200$ & 3,07100 & 4001 & 78000 \\
$\lambda$ & 0,03366 & 0,00586 & 0,00005 & 0,02404 & 0,03303 & 0,04679 & 4001 & 78000 \\
$\sigma_{V}^{2}$ & 0,32590 & 0,22260 & 0,00211 & 0,11350 & 0,26870 & 0,89020 & 4001 & 78000 \\
\hline
\end{tabular}

Note que $\sigma_{V}^{2}$ é muito pequeno o que indicaria que a componente de variância não é significativamente diferente de zero, de modo que não há efeito de clínica na parte da população de curados. 
Este resultado já tinha sido apontado na análise pelo modelo de Chen-Ibrahim-Sinha com efeitos aleatórios, apresentado na seção 4.2.

O software Winbugs, neste exemplo, executou o algoritmo Metropolis-Hastings.

A Figura 7.1 mostra os histogramas feitos com os dados gerados pelo programa Winbugs.
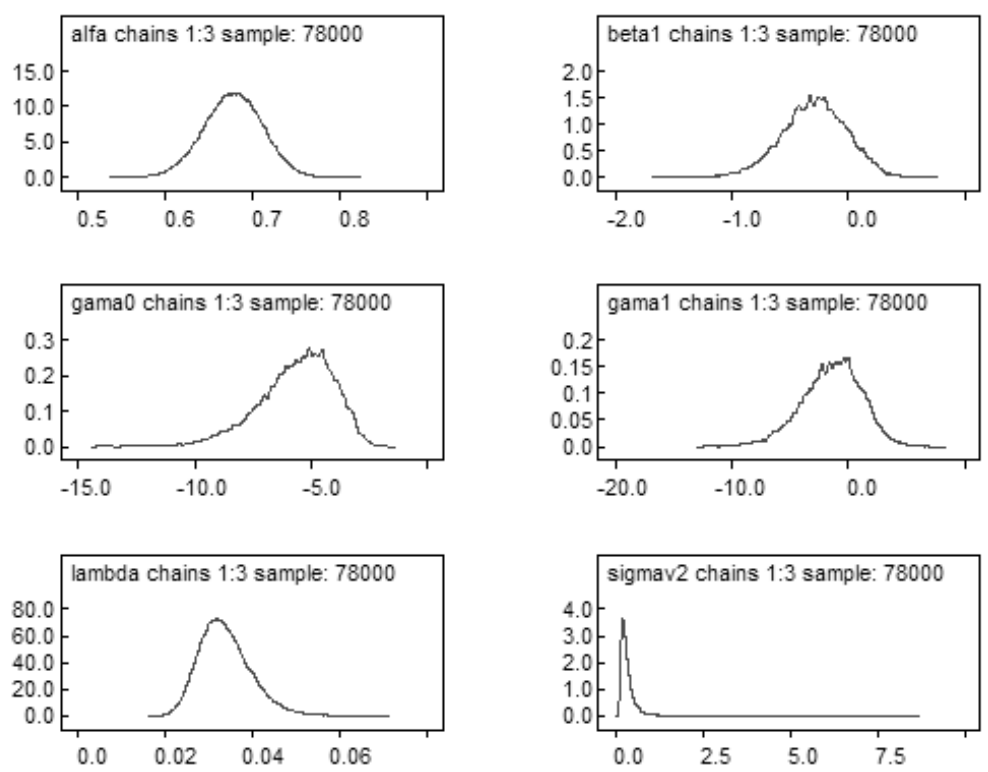

Figura 7.1: Histogramas - câncer na orofaringe - modelo de mistura com efeitos aleatórios - MetropolisHastings

A Figura 7.2 mostra os gráficos dos valores de $\sqrt{\hat{R}}$ (Gelman e Rubin, 1992), como definidos na seção 2.2 .

Note que para todos os parâmetros, os valores estão tendendo ao valor 1, o que indica que as cadeias estão convergindo. 

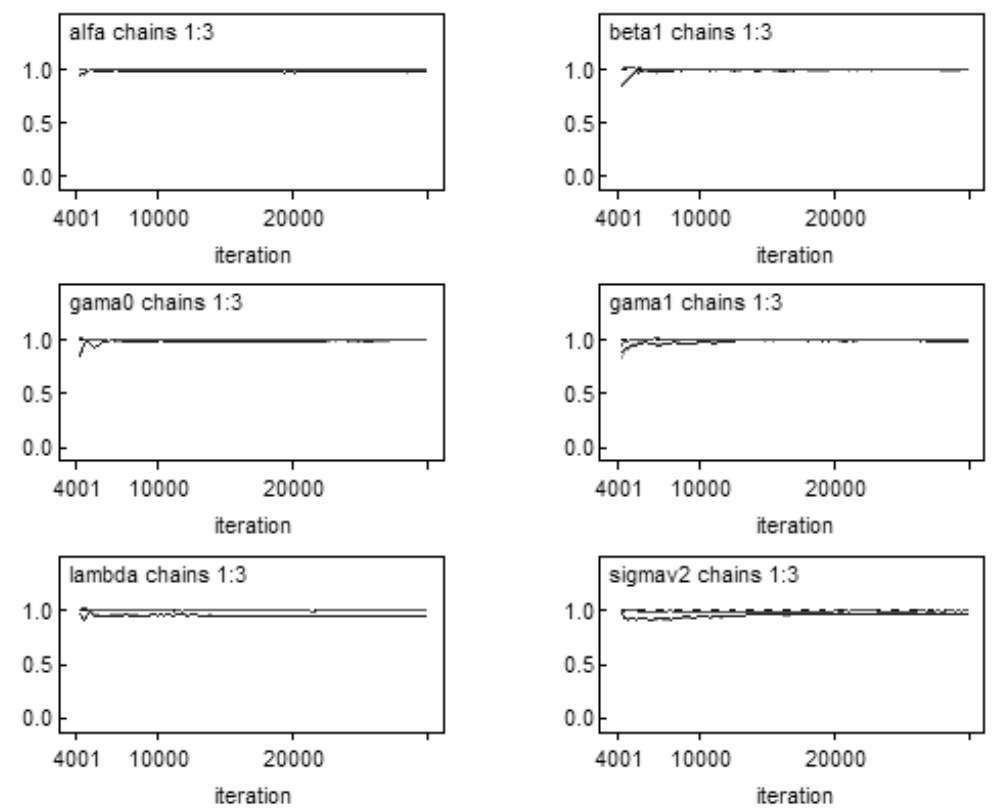

Figura 7.2: $\sqrt{\hat{R}}$ - câncer na orofaringe - modelo de mistura com efeitos aleatórios - Metropolis-Hastings

\subsection{Análise clássica}

O conjunto de dados, apresentado na seção 4.1, foi novamente utilizado sendo feita a abordagem relatada na seção 5.3. Desta vez, o software utilizado para a compilação dos dados foi Maple versão 10. Os comandos estão apresentados no apêndice A.6.

Os resultados obtidos estão na Tabela 7.2. 
Tabela 7.2: Resultados - câncer na orofaringe - modelo de mistura com efeitos aleatórios - REML

\begin{tabular}{ccc}
\hline parâmetros & estimativa & desvio-padrão \\
\hline$\alpha$ & 0,1412 & 0,0206 \\
$\lambda$ & 0,4717 & 0,4356 \\
$\beta_{1}$ & $-0,5736$ & 0,4356 \\
$\gamma_{0}$ & $-0,8482$ & 0,4154 \\
$\gamma_{1}$ & $-2,7615$ & 7,1688 \\
$\sigma_{V}^{2}$ (BLUP) & 0,0468 & - \\
$\sigma_{V}^{2}$ (REML) & 0,2872 & 0,3674 \\
\hline \multicolumn{4}{|}{} \\
\hline efeito de clínica & preditores (Blup) \\
\hline$V_{1}$ & 0,2912 & \\
$V_{2}$ & $-0,2933$ & \\
$V_{3}$ & 0,1505 & \\
$V_{4}$ & 0,0461 & \\
$V_{5}$ & $-0,2794$ & \\
$V_{6}$ & 0,0849 & \\
\hline
\end{tabular}

Note que os valores obtidos para $\sigma_{V}^{2}$, apresentados nas Tabelas 7.1 e 7.2 estão bem próximos. Em ambos, tem-se valores pequenos para a variância dos efeitos aleatórios, indicando que pode não haver diferenças entre as clínicas.

As variâncias para $\alpha$ e $\lambda$ estão subestimadas. Valores melhores para os desvios-padrão destes dois parâmetros podem ser obtidos por bootstrap (Efron, 1979; Efron, 1982).

\subsection{Conclusões}

Os resultados obtidos, para $\sigma_{V}^{2}$ com a estimação bayesiana, via Metropolis-Hastings estão próximos aos obtidos com a abordagem clássica, via REML.

É nítida uma diferença de estimativas para alguns parâmetros, fato que pode ter ocorrido devido às características dos dados utilizados, como, por exemplo, baixo número de falhas em algumas clínicas, poucas observações com estágio avançado do tumor.

Os resultados obtidos através do modelo de mistura com efeitos aleatórios para a variância dos efeitos aleatórios estão coerentes com os obtidos com o modelo de Chen-Ibrahim-Sinha com efeitos aleatórios, em termos da análise de efeito de clínica na fração de curados. 


\section{Capítulo 8}

\section{Conclusões}

Os modelos propostos nos Capítulos 2 e 5 foram analisados por duas perspectivas: bayesiana e clássica. Para avaliarmos as técnicas estudadas, foram feitos estudos de simulação, apresentados nos Capítulos 3 e 6. Para exemplificar o uso dos modelos, foram feitas análises com dados reais de um estudo de câncer na orofaringe, apresentados na seção 4.1 e cujos resultados encontram-se nos Capítulos 4 e 7.

\subsection{Resultados e conclusões}

Em todos os casos simulados para o modelo de Chen-Ibrahim-Sinha com efeitos aleatórios, os resultados tanto para as estimativas dos parâmetros quanto para os desvios-padrão foram muito bons. Em quase todas as situações, a diferença entre os valores médios estimados e os valores verdadeiros foram menores do que um desvio-padrão, o que indica que os resultados obtidos via simulação, para os parâmetros estudados, estão bons.

Além disso, tal diferença apresentou-se menor nos casos em que tínhamos mais indivíduos em cada clínica (caso $n_{k}=50$ em comparação com $n_{k}=15$ ). Tanto no caso bayesiano, quanto no clássico, o fato de termos mais indivíduos em cada clínica nos fornece valores estimados mais acurados, como esperado.

Com relação às estimativas para os desvios-padrão, as simulações mostraram que os desviospadrão das médias estão próximos aos devios-médio em praticamente todos os casos. Isso é um bom indício de que os desvios estão sendo bem estimados pelas segundas-derivadas da log-verossimilhança, ou seja, pela informação de Fisher, no caso clássico. Somente para o parâmetro $\sigma_{V}^{2}$ esta diferença é um pouco maior. Mas, este é o parâmetro cuja variância está sendo estimada por REML.

Esta proximidade entre os valores de desvio da média e médio e entre as médias das estimativas e os valores verdadeiros são um indício de que as técnicas estudadas estão adequadas para a análise do modelo proposto no Capítulo 2.

Ainda no modelo de Chen-Ibrahim-Sinha com efeitos aleatórios, é perceptível que os valores dos desvios-padrão das estimativas estão, quase sempre, menores no caso $n_{k}=50$ em comparação com 
$n_{k}=15$. A exceção ocorre no caso de $\sigma_{V}^{2}$ em que essa situação se inverte, mas a acurácia do valor estimado é melhor em $n_{k}=50$ do que em $n_{k}=15$. Isso em ambas as inferências bayesiana e clássica.

Os histogramas das estimativas indicam que os valores parecem apresentar distribuições normal. No caso clássico, este resultado é condizente com uma análise via estimadores de máxima verossimilhança.

É interessante notar que os resultados obtidos via abordagens clássica e bayesiana estão próximos, ou seja, ambos estão apresentando estimativas próximas para o modelo em estudo.

Já com relação ao modelo de mistura com efeitos aleatórios, quando analisados por MetropolisHastings, há indícios de que os valores estimados para $\sigma_{V}^{2}$ estão sendo subestimados, isso é mais aparente no caso $n_{k}=15$, em que, em alguns casos, o resultado não está bom. No caso $n_{k}=$ 50 , os resultados estão melhores. Para os demais parâmetros, os resultados das simulações estão satisfatórios.

Com relação à abordagem clássica, tem-se que os valores estimados para $\sigma_{V}^{2}$ estão, em média, subestimados quando o valor verdadeiro é 1 , e superestimados, quando seu valor verdadeiro é 0,5 . Essa diferença é mais aparente no caso $n_{k}=15$, mas ainda assim os resultados estão satisfatórios. Como esperado, os resultados para todos os parâmetros estão melhores no caso $n_{k}=50$ em que todos os grupos de parâmetros simulados apresentam resultados bons.

Levando-se em conta o modelo de mistura com efeitos aleatórios, o melhor resultado apresentado nas simulações foi obtido através da abordagem clássica, especialmente quando o número de indivíduos por clínica é maior.

Comparando as simulações tanto para o modelo de mistura quanto para o modelo de ChenIbrahim-Sinha com efeitos aleatórios, percebe-se que os resultados estão melhores quando se tem mais indivíduos em cada clínica. De todos os casos estudados via simulação, o que apresentou o pior resultado foi o modelo de mistura com efeitos aleatórios, quando analisado por Metropolis-Hastings. Nos demais casos, os resultados estão dentro do esperado.

\subsection{Perspectivas futuras}

Devido às características do modelo estudado no Capítulo 2, os efeitos aleatórios não puderam entrar separadamente nos curados e não-curados, para um mesmo modelo, como feito em Yau e Ng (2001). Por uma questão de não identificabilidade do modelo, foram colocados efeitos aleatórios no modelo de Chen-Ibrahim-Sinha somente para a parcela de curados. Em um próximo passo, analisaremos o caso em que o efeito aleatório será estudado para toda a população.

Com relação à abordagem clássica uma alternativa aos BLUP e REML seria utilizar o algoritmo EM para a estimação. O novo resultado poderá ser utilizado para compararmos os resultados das estimativas. Esta nova abordagem poderá ser utilizada tanto no modelo de mistura quanto no modelo de Chen-Ibrahim-Sinha com efeitos aleatórios. 
Com relação à abordagem bayesiana uma alternativa ao uso do programa BUGS seria a utilização do pacote UMACS do R ou a programação direta do Metropolis-Hastings. Os resultados poderão ser utilizados para compararmos a acertividade dos diversos softwares.

Para o modelo de mistura com efeitos aleatórios, em um próximo passo, pretendemos utilizar bootstrap de Efron (Efron, 1979; Efron, 1982) para a obtenção dos valores dos desvios-padrão para $\alpha$ e $\lambda$.

Ainda no modelo de mistura, pode ser feita uma análise semi-paramétrica para a estimação da função de risco basal $h_{20}(t)$, como alternativa ao uso da distribuição Weibull que adotamos.

Como alternativa ao uso dos resultados das simulações para a comparação dos modelos de mistura e de Chen-Ibrahim-Sinha com efeitos aleatórios, pretendemos estudar técnicas de comparação de modelos. Ressalto que estas comparações têm que ser feitas com muito cuidado, visto que os parâmetros têm interpretações diferentes nos dois modelos.

Há dois artigos de Cancho; Rodrigues \& Castro (2008) e de Castro; Cancho \& Rodrigues (2007), em desenvolvimento, em que o modelo de Chen-Ibrahim-Sinha é trabalhado utilizando distribuição binomial negativa no lugar da distribuição de Poisson. Pretendemos analisar como fica este modelo quando inserimos efeitos aleatórios.

Finalmente, pretendemos analisar como estes dois modelos apresentados aqui poderão ser analisados com a "teoria unificada para modelos de longa duração", que foi publicada em uma versão por Yin e Ibrahim (2005) e, em um outro enfoque, submetida para publicação por Rodrigues e Louzada-Neto (2006). 


\section{Apêndice A}

\section{Programas}

\section{A.1 Gerando os dados para a simulação - modelo de Chen-Ibrahim-Sinha com efeitos aleatórios}

A seguir está descrito o procedimento que foi feito para que o programa $\mathrm{R}$ (versão 1.19.2.3) gerasse os dados a serem utilizados na simulação para o modelo de Chen-Ibrahim-Sinha com efeitos aleatórios.

1. Foram atribuídos valores para os parâmetros, para o número de clínicas, para o número de pacientes por clínica e para a censura, conforme apresentados no Capítulo 3;

2. para as covariáveis $X$, foram atribuídos, aleatoriamente, valores 0 e 1, na proporção 2:1;

3. os efeitos aletórios $V_{k}$, para cada uma das $k$ clínicas foram sorteados de uma distribuição normal, com média 0 e variância $\sigma_{V}^{2}$;

4. para cada indivíduo de cada uma das clínicas, foram sortedaos valores para $N_{j k}$, a partir da distribuição de Poisson de média $\exp \left(\gamma_{0}+\gamma_{1} * x_{j k}+V_{k}\right)$;

5. para cada célula de 0 a $N_{j k}$, foi armazenado um valor para $z_{j k}$, sorteado de uma Weibull, com parâmetros $\alpha$ e $\exp \left(-\beta * x_{j k}\right)$;

6. $T_{j k}$ armazenou o valor mínimo entre todos os $z_{j k}$;

7. se o valor dde censura fosse menor do que $T_{j k}$ então $T_{j k}$ passa a ter o valor da censura e, neste caso, o indicador de censura recebe o valor 0 .

A seguir estão os principais comandos, feitos para o progarama $R$, que foi utilizado para gerar os dados. A variável "listpar" recebeu os valores dos parâmetros.

for (ipar in 1:totallinhaslistpar) \{ 


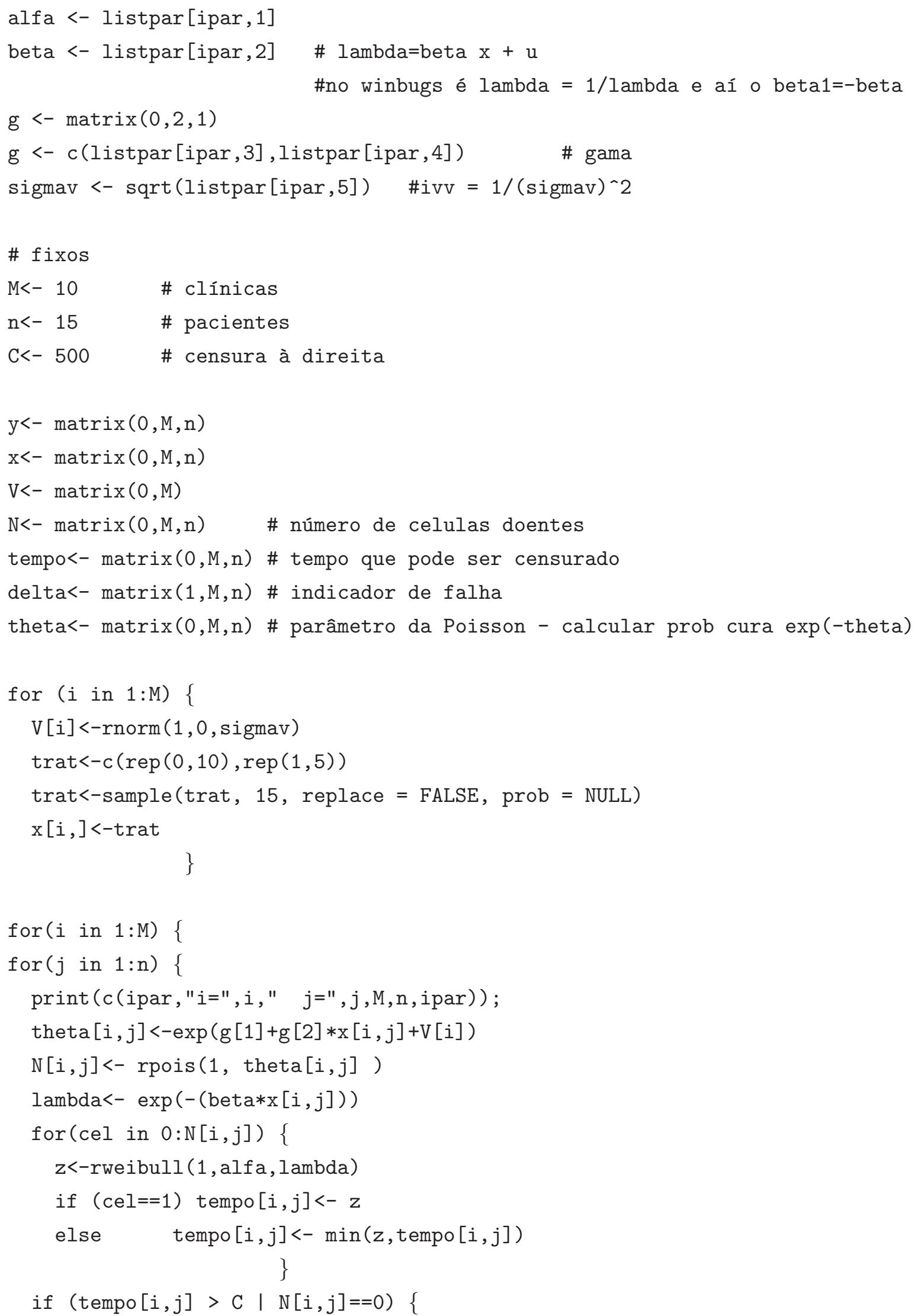




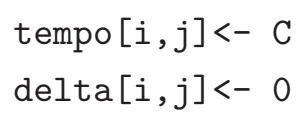

\section{A.2 Comandos em Winbugs - modelo de Chen-Ibrahim-Sinha com efeitos aleatórios}

Nesta seção estão apresentados os comandos para o modelo 2.2 utilizado no programa Winbugs (versão 1.4), relatados na seção 4.2 e na simulação apresentada na seção 3.2.

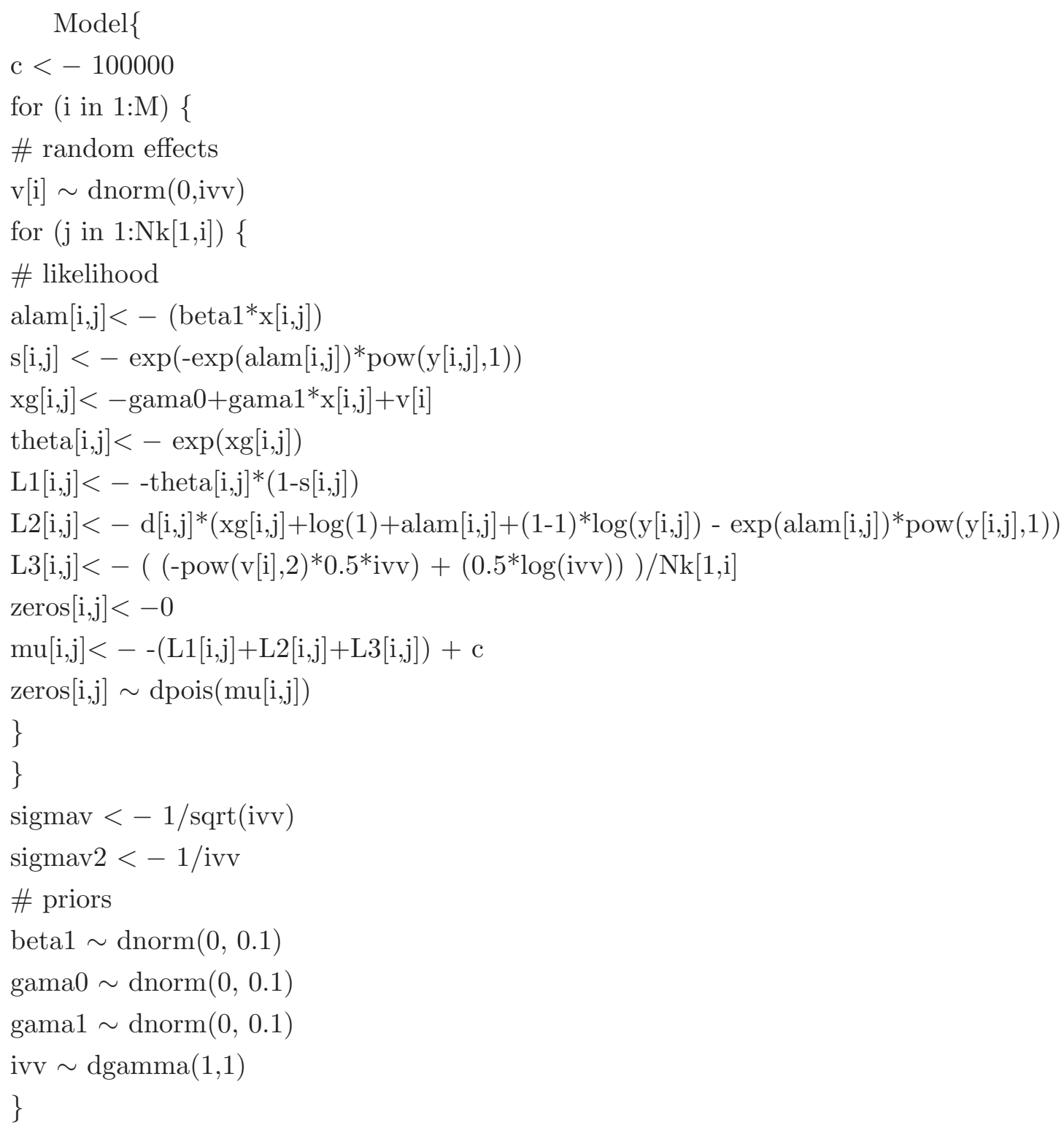

Os dados da Tabela 4.1 entraram do seguinte modo: 
$\operatorname{list}(\mathrm{M}=6, \mathrm{Nk}=\operatorname{structure}(. \operatorname{Data}=\mathrm{c}(9,14,16,7,11,9), . \mathrm{Dim}=\mathrm{c}(1,6))$,

$\mathrm{y}=$ structure $($. Data $=\mathrm{c}(1312,1219,264,11,170,372,182,723,90,-1,-1,-1,-1,-1,-1,-1,270,162$, $228,370,805,517,599,89,99,219,154,266,407,751,-1,-1,184,1064,1472,526,1495,661,407$, $548,1317,1317,1307,800,666,1060,465,518,235,324,1565,1234,293,593,213,-1,-1,-1,-1,-1$, $-1,-1,-1,-1,245,262,915,1377,172,15,382,363,107,757,38,-1,-1,-1,-1,-1,222,1644,785,11$, 1095, 914, 346, 608, 112, -1, -1, -1, -1, -1, -1, -1), .Dim=c(6,16)),

$\mathrm{d}=$ structure $($ Data $=\mathrm{c}(0,0,1,1,1,1,0,0,0,-1,-1,-1,-1,-1,-1,-1,1,1,1,1,1,1,1,1,1,1,1,1,1$, $0,-1,-1,1,1,0,1,0,1,1,1,0,0,0,1,1,0,1,1,1,1,0,0,1,0,1,-1,-1,-1,-1,-1,-1,-1,-1,-1,1,1$, $1,0,1,1,1,1,1,1,1,-1,-1,-1,-1,-1,1,0,1,1,0,0,1,1,1,-1,-1,-1,-1,-1,-1,-1)$, .Dim=c $(6,16))$, $\mathrm{x}=$ structure $($ Data $=\mathrm{c}(0,0,1,1,0,0,0,1,0,-1,-1,-1,-1,-1,-1,-1,0,0,1,1,1,0,0,1,0,1,1$, $1,0,0,-1,-1,0,0,0,0,0,0,0,0,0,0,0,0,0,0,0,0,0,0,0,0,0,1,0,-1,-1,-1,-1,-1,-1,-1$, $-1,-1,1,0,0,1,1,1,1,0,1,1,0,-1,-1,-1,-1,-1,0,0,0,1,0,0,0,0,0,-1,-1,-1,-1,-1,-1,-1)$, $. \operatorname{Dim}=c(6,16)))$

\section{A.3 Comandos em Maple - modelo de Chen-Ibrahim-Sinha com efeitos aleatórios}

Nesta seção estão apresentados os comandos referentes ao modelo de mistura com efeitos aleatórios apresentado no Capítulo 5 utilizado no programa Maple (versão 10), relatados na seção 4.3 e na simulação apresentada na seção 3.3 .

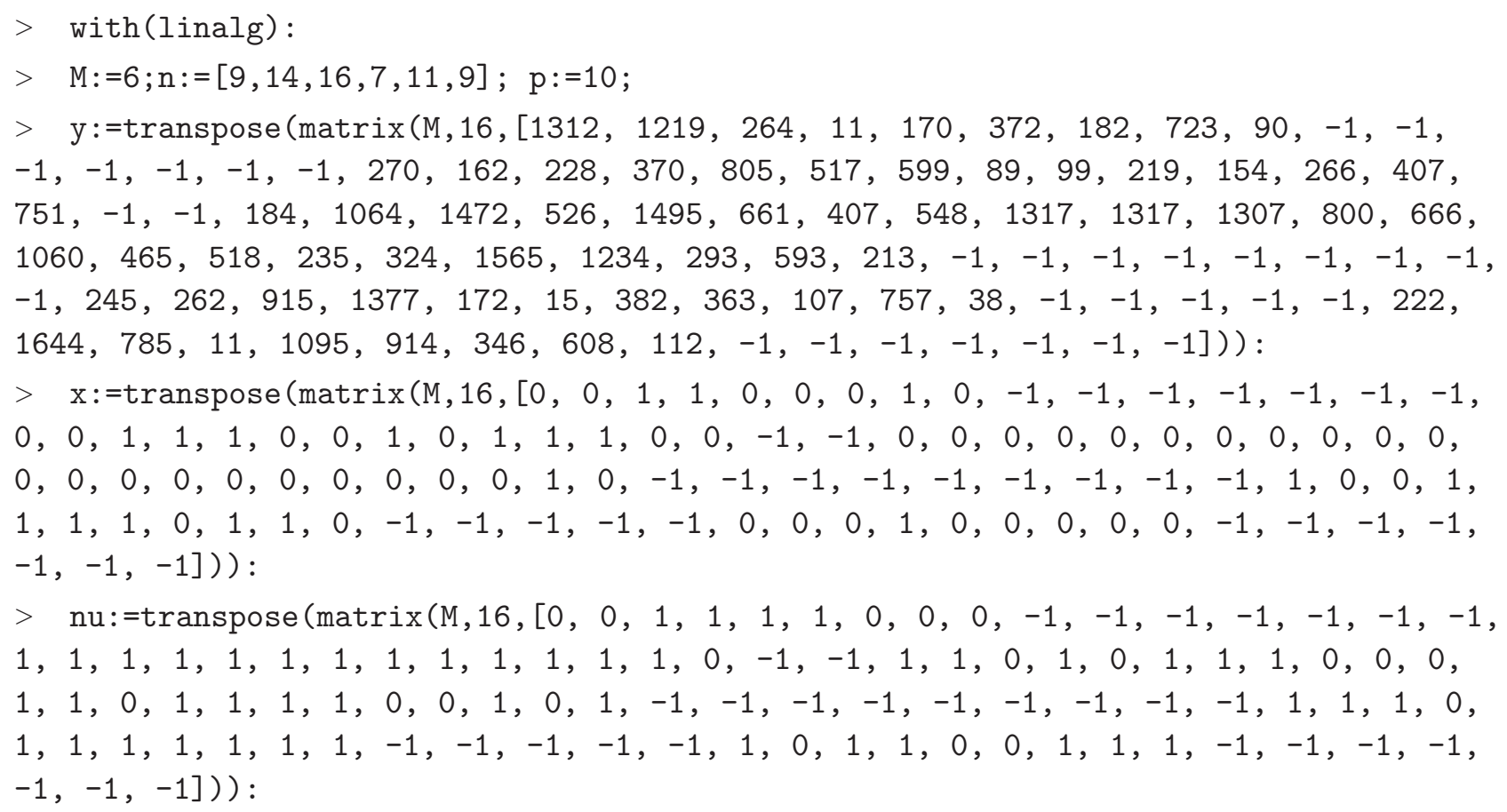

Contas intermediárias para facilitar a definição de l, a log-verossimilhança completa, já somada em N

$>f 1:=-1 / 2 * \ln (2 * \mathrm{Pi} * \operatorname{sigmav} 2)-\left(\mathrm{V}[\mathrm{K}]^{\wedge} 2\right) /(2 * \operatorname{sigmav} 2)$; 


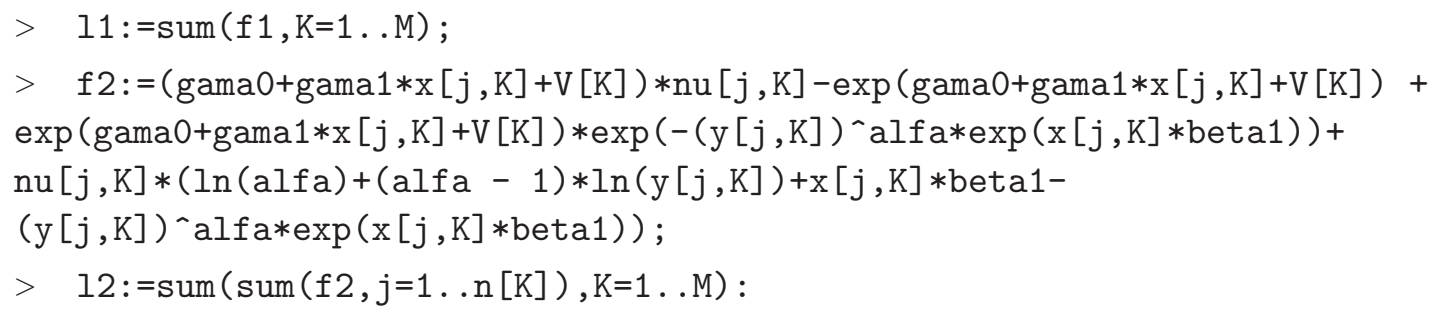

Definindo a matriz hessiana

$>\mathrm{H}:=\operatorname{hessian}(1,[a l f a, \operatorname{beta1}$, gama0, gama1, V[1], V [2], V[3], V[4], V[5], V [6] ]):

$>\mathrm{H} 2:=\operatorname{matrix}(\mathrm{p}, \mathrm{p})$;

Calculando a norma da diferença de phi em dois estágios consecutivos

$>\operatorname{difphi}:=\operatorname{evalf}(\operatorname{norm}(\operatorname{col}(\operatorname{phi}, 1)-\operatorname{col}(\operatorname{phi}, 2), 2))$;

$>$ passo $:=0.1$;

$>$ for $z$ from 1 to $M$ do $\operatorname{dV}[z]:=\operatorname{diff}(1, V[z])$; end do:

Programando o "laço" para a iteração

$>$ for $t$ from 1 to 1000 while (difphi > 0.001) do

for a from 1 to $p$ do

phi $[a, 1]:=\operatorname{phi}[a, 2]$ :

end do;

alfa:=phi $[1,1]$; beta1:=phi $[2,1] ; \operatorname{gama} 0:=\operatorname{phi}[3,1] ; \operatorname{gama1}:=\operatorname{phi}[4,1]$;

$\mathrm{V}[1]:=\operatorname{phi}[5,1] ; \mathrm{V}[2]:=\operatorname{phi}[6,1] ; \mathrm{V}[3]:=\operatorname{phi}[7,1] ; \mathrm{V}[4]:=\operatorname{phi}[8,1]$;

$\mathrm{V}[5]:=\operatorname{phi}[9,1] ; \mathrm{V}[6]:=\operatorname{phi}[10,1]$;

for a from 1 to $p$ do

$\mathrm{U} 2[a, 1]:=\operatorname{evalf}(\operatorname{subs}(\{\operatorname{alfa}=\operatorname{phi}[1,1]$, beta1=phi $[2,1]$,gama $0=\operatorname{phi}[3,1]$, gama1=phi $[4,1]$, sigmav2=sigmav2, $V[1]=\operatorname{phi}[5,1], V[2]=\operatorname{phi}[6,1]$, $\mathrm{V}[3]=\operatorname{phi}[7,1], \mathrm{V}[4]=\operatorname{phi}[8,1], \mathrm{V}[5]=\operatorname{phi}[9,1], \mathrm{V}[6]=\operatorname{phi}[10,1]\}, \mathrm{U}[\mathrm{a}, 1]))$; end do; 
for $b$ from 1 to $p$ do

$\mathrm{H} 2[\mathrm{a}, \mathrm{b}]:=\operatorname{evalf}(\operatorname{subs}(\{\mathrm{alfa}=\operatorname{phi}[1,1]$, beta1=phi $[2,1]$,gama0=phi $[3,1]$, gama1=phi $[4,1]$, sigmav2=sigmav2, $V[1]=\operatorname{phi}[5,1], \mathrm{V}[2]=\operatorname{phi}[6,1]$, $\mathrm{V}[3]=\operatorname{phi}[7,1], \mathrm{V}[4]=\operatorname{phi}[8,1], \mathrm{V}[5]=\operatorname{phi}[9,1], \mathrm{V}[6]=\operatorname{phi}[10,1]\}, \mathrm{H}[\mathrm{a}, \mathrm{b}]))$; end do;

end do;

for a from 1 to $p$ do

$\operatorname{phi}[\mathrm{a}, 2]$ := phi $[\mathrm{a}, 1]-\operatorname{passo*}(\operatorname{evalm}(\operatorname{inverse}(\mathrm{H} 2)$ \&* (U2)) $[\mathrm{a}, 1])$;

end do;

sigmav2:= sum('V[z] '2', 'z'=1..M) / M;

\# atualiza a condição de parada para a convergência

difphi:=evalf $(\operatorname{norm}(\operatorname{col}(p h i, 1)-\operatorname{col}(p h i, 2), 2))$;

print (V, sigmav2);

print ("t=", $t)$;

end do;

Lendo da segunda coluna de phi quem são os "chapéus", as estimativas de $\alpha, \beta_{1}, \gamma_{0}, \gamma_{1}$ e os BLUP para $\mathbf{V}$. Lendo também o valor estimado para $\sigma_{V}^{2}$ por este método

$>$ alfachapeu:=phi $[1,2]$; beta1chapeu:=phi $[2,2]$; gama0chapeu:=phi $[3,2]$; gama1chapeu:=phi $[4,2] ; \mathrm{V}[1]:=\operatorname{phi}[5,2] ; \mathrm{V}[2]:=\operatorname{phi}[6,2] ; \mathrm{V}[3]:=\operatorname{phi}[7,2]$; $\mathrm{V}[4]:=\operatorname{phi}[8,2] ; \mathrm{V}[5]:=\operatorname{phi}[9,2] ; \mathrm{V}[6]:=\operatorname{phi}[10,2]$;

sigmav2chapeublup:= sum('V[z]`2', 'z'=1..M) / M;

Atualizando a matriz hessiana para os valores dos chapéus

$>$ for a from 1 to $p$ do

for $b$ from 1 to $p$ do

$\mathrm{H} 2[\mathrm{a}, \mathrm{b}]:=\operatorname{evalf}(\operatorname{subs}(\{\operatorname{alfa}=\operatorname{phi}[1,2]$, beta1=phi $[2,2]$, gama0=phi $[3,2]$, gama1=phi $[4,2]$, sigmav2=sigmav2chapeublup, $V[1]=p h i[5,2]$, $\mathrm{V}[2]=\operatorname{phi}[6,2], \mathrm{V}[3]=\operatorname{phi}[7,2], \mathrm{V}[4]=\operatorname{phi}[8,2], \mathrm{V}[5]=\operatorname{phi}[9,2]$, $\mathrm{V}[6]=\operatorname{phi}[10,2]\}, \mathrm{H}[\mathrm{a}, \mathrm{b}]))$;

end do;

end do;

REML: estimar o $\sigma_{V}^{2}$ de modo menos viesado do que pelo método acima Selecionando a parte da matriz hessiana correspondente a $\mathrm{V}$

$>\mathrm{A} 33:=\operatorname{matrix}(6,6,[])$;

$>$ H2inv:=inverse(H2):

$>A 33:=\operatorname{evalm}(-1 * \operatorname{matrix}([\operatorname{col}(\operatorname{matrix}([\operatorname{col}(\mathrm{H} 2 \mathrm{inv}, 5 \ldots 10)]), 5 \ldots 10)]))$;

$>\operatorname{sigmav} 2 \operatorname{chapeu}:=1 / \mathrm{M} * \operatorname{evalm}(\operatorname{trace}(\mathrm{A} 33)+(\operatorname{transpose}(\mathrm{V}) \& * \mathrm{~V}))$;

Variância de sigmav2chapeu, pelo REML

$>\operatorname{varsigma2}:=1 /(2 *(1 /(\operatorname{sigmav} 2$ chapeu $) \sim 2 *(\mathrm{M}-2 / \operatorname{sigmav} 2$ chapeu*trace $(\mathrm{A} 33))+$ 1/( igmav2chapeu )^4*trace (evalm (A33^2)))) ;

Calculando as variâncias de $\alpha, \beta_{1}, \gamma_{0}, \gamma_{1}$ 
$>\operatorname{var}:=\operatorname{evalm}(-1 * \operatorname{matrix}([\operatorname{col}(\operatorname{matrix}([\operatorname{col}(H 2 i n v, 1 \ldots 4)]), 1.4)]))$;

\section{A.4 Gerando os dados para a simulação - modelo de mistura com efeitos aleatórios}

A seguir está descrito o procedimento que foi feito para que o programa $\mathrm{R}$ (versão 1.19.2.3) gerasse os dados a serem utilizados na simulação para o modelo de mistura com efeitos aleatórios.

1. Foram atribuídos valores para os parâmetros, para o número de clínicas, para o número de pacientes por clínica e para a censura, conforme apresentados no Capítulo 6;

2. para as covariáveis $X$, foram atribuídos, aleatoriamente, valores 0 e 1, na proporção 2:1;

3. os efeitos aletórios $V_{k}$, para cada uma das $k$ clínicas foram sorteados de uma distribuição normal, com média 0 e variância $\sigma_{V}^{2}$;

4. para cada indivíduo de cada uma das clínicas, foram armazenados valores para $\pi_{j k}$, dados por $1 /\left(1+\exp \left(\gamma_{0}+\gamma_{1} * x_{j k}+V_{k}\right)\right)$

5. para decidir se o indivíduo $j$ da clínica $k$ seria curado ou não, foi feito um sorteio a partir de uma distribuição binomial com probabilidade $\pi_{j k}$ de o indivíduo ser não curado;

6. os indivíduos sorteados com não-curados receberam $T_{j k}$ igual a censura;

7. os indivíduos sorteados com curados receberam $T_{j k}$ um valor $\left(-\ln (1-u) / \lambda * \exp \left(\eta_{j k}\right)\right)^{1 / \alpha}$ em que $u$ foi sorteado da distribuição uniforme $(0,1)$.

A seguir estão os principais comandos, feitos para o progarama $R$, que foi utilizado para gerar os dados. A variável "listpar" recebeu os valores dos parâmetros.

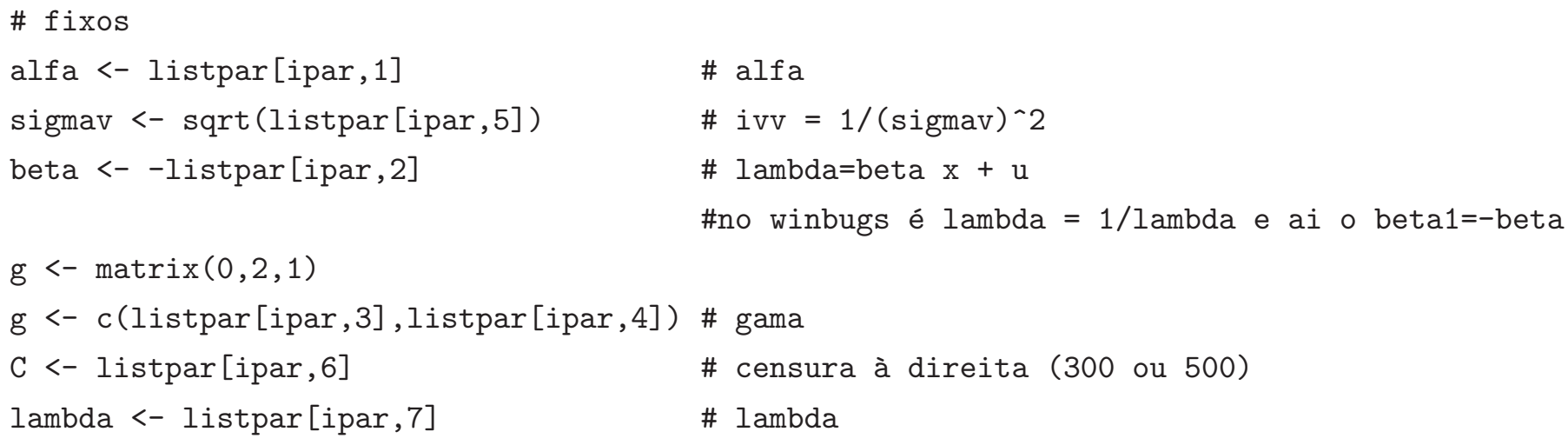




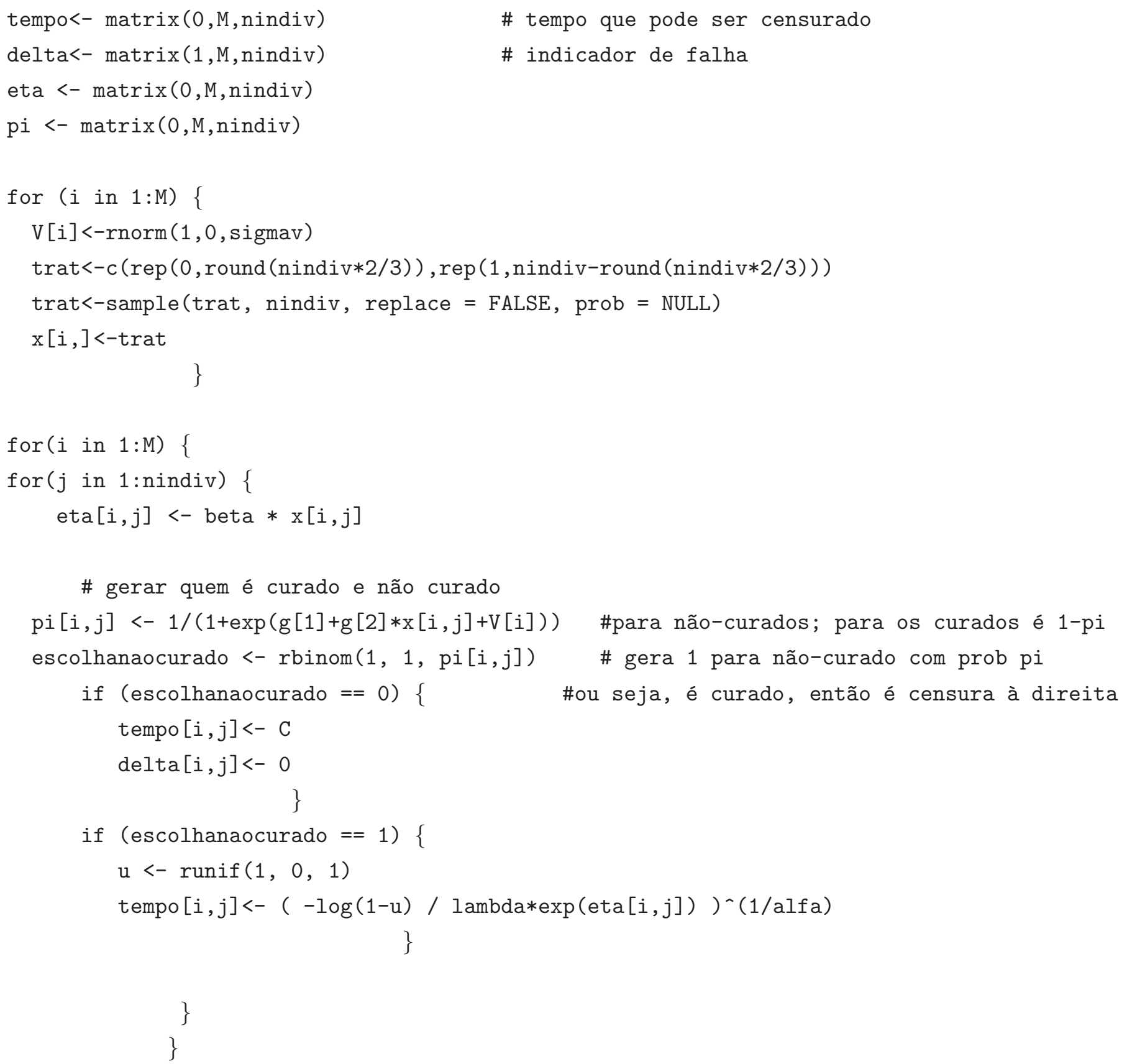

\section{A.5 Comandos em Winbugs - modelo de mistura com efeitos aleatórios}

Nesta seção estão apresentados os comandos utilizados na abordagem bayesiana para o modelo de mistura com efeitos aleatórios utilizado no programa Winbugs (versão 1.4), relatados na seção 5.2 e na simulação apresentada na seção 6.2.

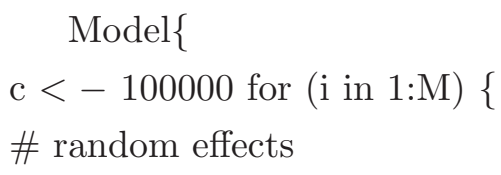




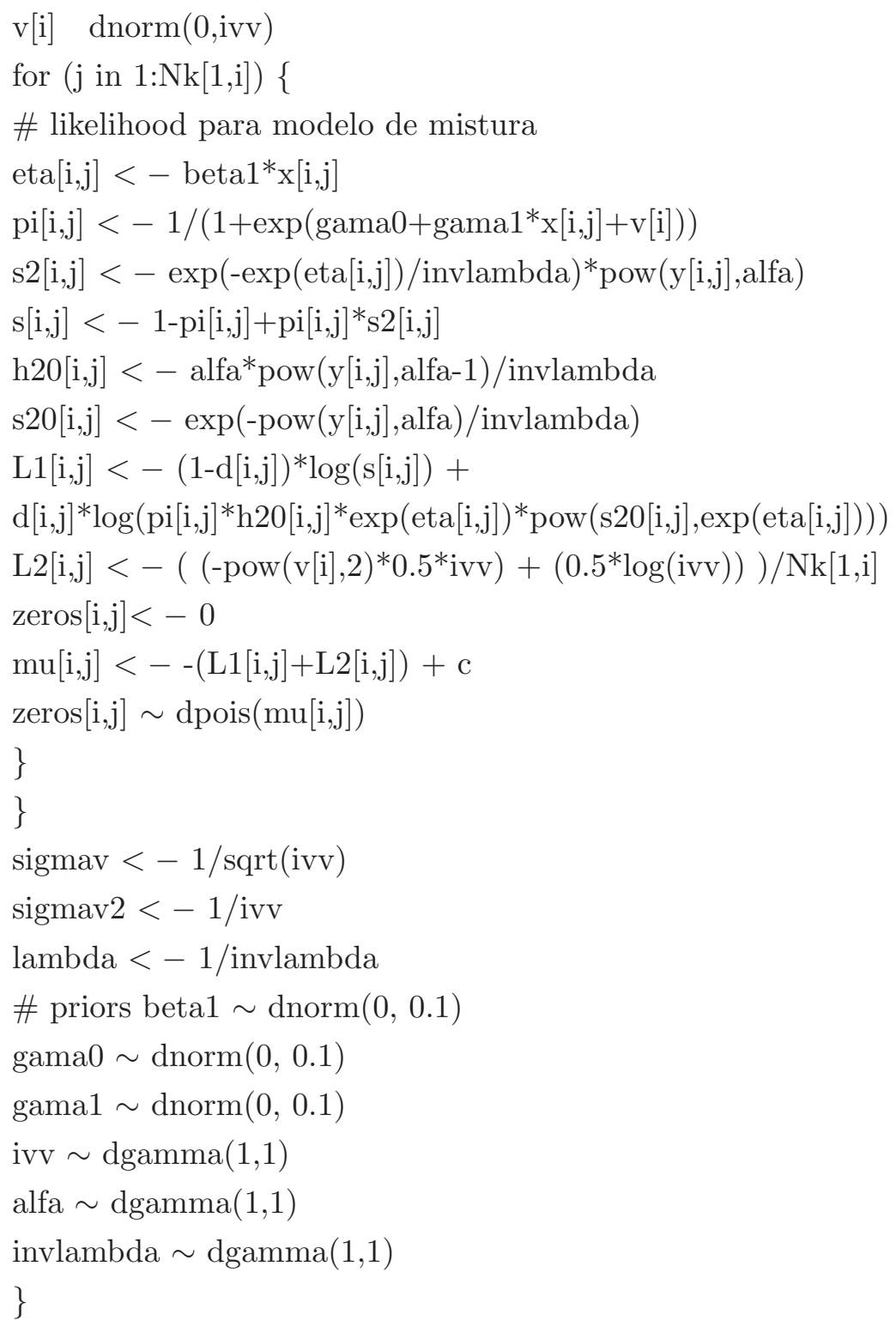

\section{A.6 Comandos em Maple - modelo de mistura com efeitos aleatórios}

Nesta seção estão apresentados os comandos utilizados na abordagem clássica para o modelo de mistura com efeitos aleatórios utilizado no programa Maple (versão 10), relatados na seção 5.3 e na simulação apresentada na seção 6.3 .

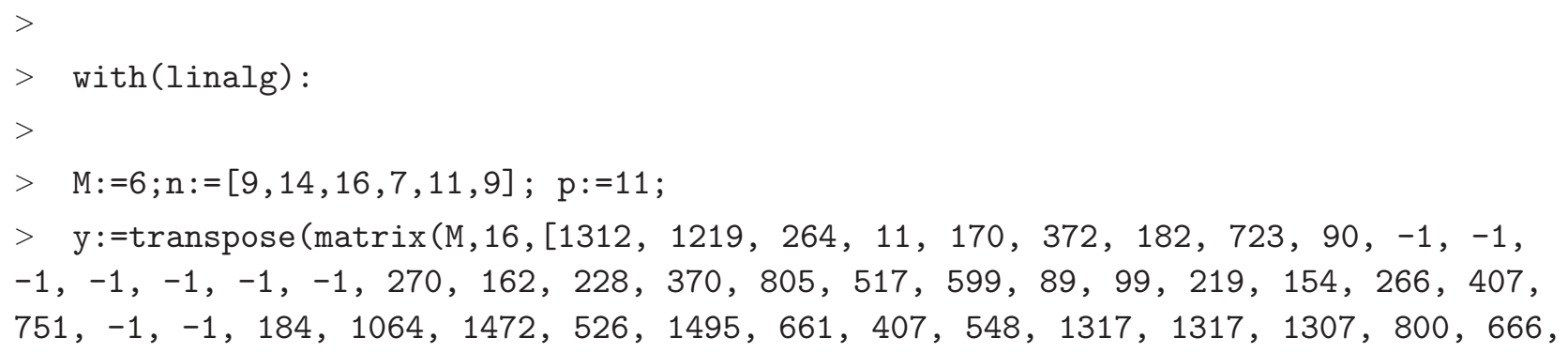


$1060,465,518,235,324,1565,1234,293,593,213,-1,-1,-1,-1,-1,-1,-1,-1$, $-1,245,262,915,1377,172,15,382,363,107,757,38,-1,-1,-1,-1,-1,222$, $1644,785,11,1095,914,346,608,112,-1,-1,-1,-1,-1,-1,-1]))$ :

$>\mathrm{x}:=$ transpose $(\operatorname{matrix}(\mathrm{M}, 16,[0,0,1,1,0,0,0,1,0,-1,-1,-1,-1,-1,-1,-1$, $0,0,1,1,1,0,0,1,0,1,1,1,0,0,-1,-1,0,0,0,0,0,0,0,0,0,0,0$, $0,0,0,0,0,0,0,0,0,0,1,0,-1,-1,-1,-1,-1,-1,-1,-1,-1,1,0,0,1$, $1,1,1,0,1,1,0,-1,-1,-1,-1,-1,0,0,0,1,0,0,0,0,0,-1,-1,-1,-1$, $-1,-1,-1]))$ :

$>\mathrm{nu}:=\operatorname{transpose}(\operatorname{matrix}(\mathrm{M}, 16,[0,0,1,1,1,1,0,0,0,-1,-1,-1,-1,-1,-1,-1$, $1,1,1,1,1,1,1,1,1,1,1,1,1,0,-1,-1,1,1,0,1,0,1,1,1,0,0,0$, $1,1,0,1,1,1,1,0,0,1,0,1,-1,-1,-1,-1,-1,-1,-1,-1,-1,1,1,1,0$, $1,1,1,1,1,1,1,-1,-1,-1,-1,-1,1,0,1,1,0,0,1,1,1,-1,-1,-1,-1$, $-1,-1,-1]))$ :

Contas intermediárias para facilitar a definição de l, a log-verossimilhança completa, já somada em N

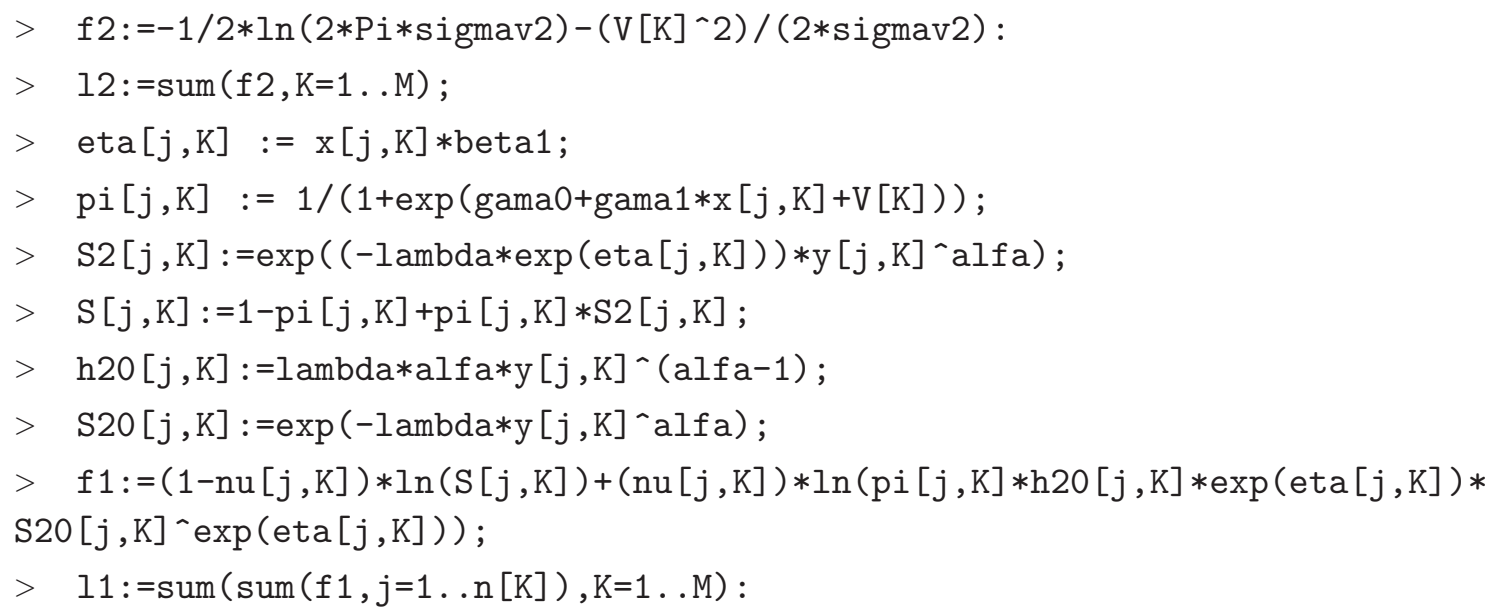

Aqui abaixo está a definição da log-verossimilhança completa

$>1:=11+12$ :

Definindo as dimensões de $\mathrm{V}$ e de sua derivada

$>\mathrm{V}:=\operatorname{array}(1 \ldots \mathrm{M}): \mathrm{dV}:=\operatorname{array}(1 \ldots \mathrm{M}):$

Definir os valores iniciais para os parâmetros, que entram na segunda coluna; a primeira coluna tem valores "grandes" o suficiente para que o difphi entre no loop. Vou sair com alfa(primeiro) e lambda(último) pré fixados, conhecidos

$>$ phi $:=\operatorname{matrix}(\mathrm{p}, 2,[1.26,1.26,10,0.6,10,-1,10,-0.8,10,0.5,10,0.5,10$,

$0.5,10,-0.5,10,-0.5,10,-0.5,0.0004,0.0004]): \quad$ sigmav $2:=0.3$ :

Definir vetor de derivadas

$>\mathrm{U}:=\operatorname{matrix}(\mathrm{p}-2,1,[\operatorname{diff}(1, \operatorname{beta} 1), \operatorname{diff}(1, \operatorname{gama} 0), \operatorname{diff}(1, \operatorname{gama} 1)$, $\operatorname{diff}(1, V[1]), \operatorname{diff}(1, V[2]), \operatorname{diff}(1, V[3]), \operatorname{diff}(1, V[4]), \operatorname{diff}(1, V[5])$, $\operatorname{diff}(1, V[6])])$ :

$>\mathrm{U} 2:=\operatorname{matrix}(\mathrm{p}-2,1)$ :

Definindo a matriz hessiana 


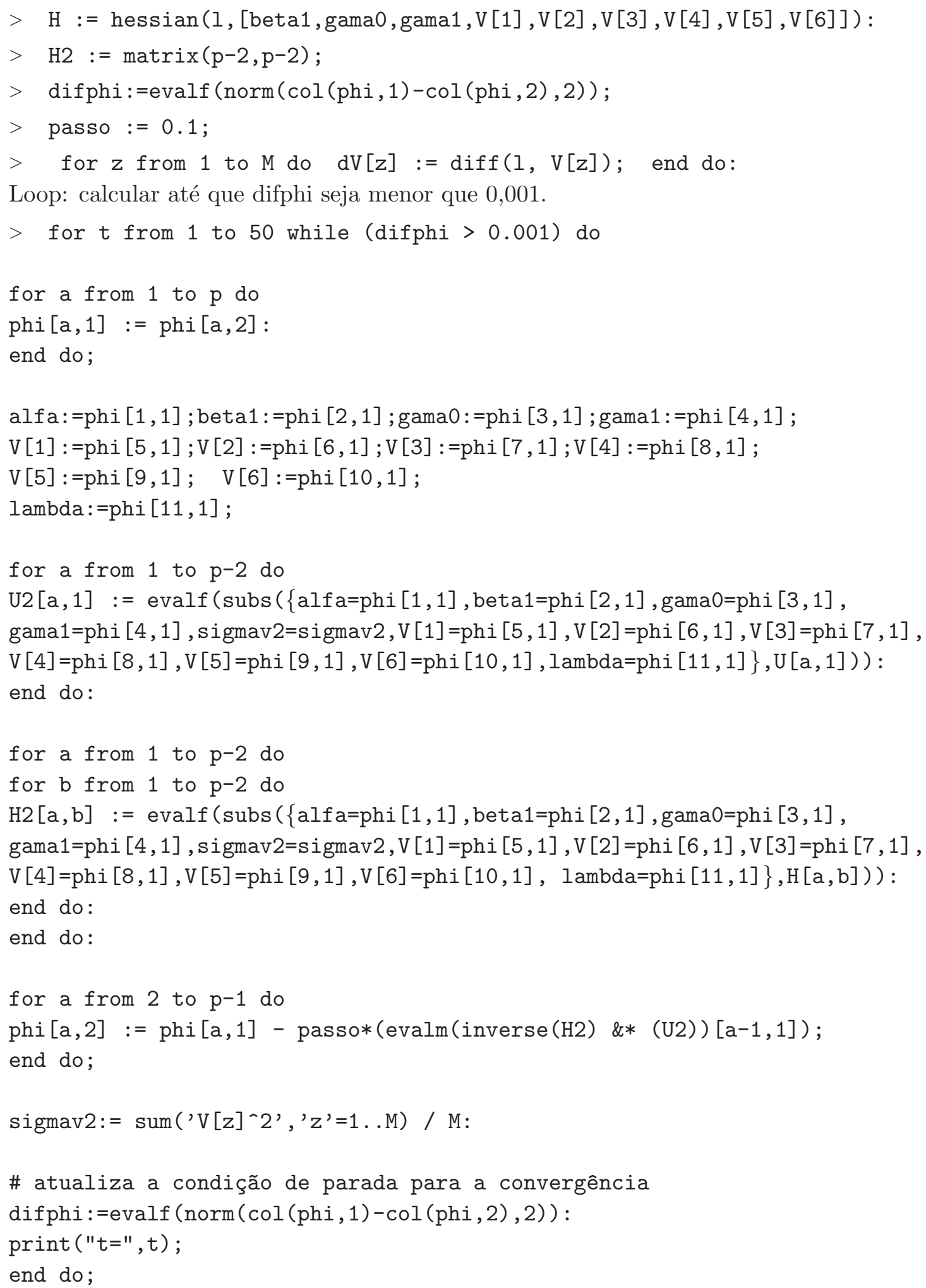


Lendo da segunda coluna de phi quem são os "chapéus", os estimadores de alfa, beta1, gama0, gama1 e os BLUP para os V.

$>$ alfachapeu:=phi $[1,2]$; beta1chapeu:=phi $[2,2]$; gama0chapeu:=phi $[3,2]$;

gama1chapeu:=phi $[4,2] ; \mathrm{V}[1]:=\operatorname{phi}[5,2] ; \mathrm{V}[2]:=\operatorname{phi}[6,2] ; \mathrm{V}[3]:=\operatorname{phi}[7,2]$;

$\mathrm{V}[4]:=\operatorname{phi}[8,2] ; \mathrm{V}[5]:=\operatorname{phi}[9,2] ; \mathrm{V}[6]:=\operatorname{phi}[10,2] ;$ lambdachapeu:=phi $[11,2]$;

sigmav2chapeublup: $=\operatorname{sum}\left({ }^{\prime} V[z]^{\prime} 2^{\prime},{ }^{\prime} z^{\prime}=1 \ldots M\right) / M$;

Atualizando a matriz hessiana para os valores dos "chapéus"

$>$ for a from 1 to $\mathrm{p}-2$ do

for $b$ from 1 to $p-2$ do

$\mathrm{H} 2[\mathrm{a}, \mathrm{b}]:=\operatorname{evalf}(\operatorname{subs}(\{\operatorname{alfa}=\operatorname{phi}[1,2]$, beta1=phi $[2,2]$, gama $0=\operatorname{phi}[3,2]$,

gama1=phi $[4,2]$, sigmav2=sigmav2chapeublup, $V[1]=\operatorname{phi}[5,2], V[2]=\operatorname{ph} i[6,2]$,

$\mathrm{V}[3]=\operatorname{phi}[7,2], \mathrm{V}[4]=\operatorname{phi}[8,2], \mathrm{V}[5]=\operatorname{phi}[9,2], \mathrm{V}[6]=\operatorname{phi}[10,2]$,

lambda=phi $[11,2]\}, \mathrm{H}[\mathrm{a}, \mathrm{b}]))$;

end do;

end do;

REML: estimar o sigmav2 de modo menos viesado do que pelo método acima

$>$ A33:=matrix $(\mathrm{M}, \mathrm{M},[])$;

$>$ H2inv:=inverse(H2):

$>\mathrm{A} 33:=\operatorname{evalm}(-1 * \operatorname{matrix}([\operatorname{col}(\operatorname{matrix}([\operatorname{col}(\mathrm{H} 2 \mathrm{inv}, 4 \ldots \mathrm{p}-2)]), 4 \ldots \mathrm{p}-2)]))$;

$>$ sigmav2chapeu:=1/M*evalm ( $\operatorname{trace}(\mathrm{A} 33)+(\operatorname{transpose}(\mathrm{V}) \& * \mathrm{~V}))$;

Substituindo os valores das estimativas no verossimilhança, que agora vai depender só de alfa e lambda, para podermos estimar alfa e lambda

$>$ \#subs (beta1=beta1chapeu, subs (gama0=gama0chapeu, subs (gama1= gama1chapeu, $\operatorname{subs}(V[1]=\operatorname{phi}[5,2], \operatorname{subs}(V[2]=\operatorname{phi}[6,2], \operatorname{subs}(V[3]=\operatorname{ph} i[7,2]$, $\operatorname{subs}(V[4]=\operatorname{phi}[8,2], \operatorname{subs}(V[5]=\operatorname{phi}[9,2], \operatorname{subs}(V[6]=\operatorname{phi}[10,2]$, $\operatorname{subs}(\operatorname{sigmav2}=\operatorname{sigmav2}$ chapeu, 1 )))) ))))));

Definir a log-verossimilhança já com as estimativas substituídas, de modo a ficarmos somente com alfa e lambda

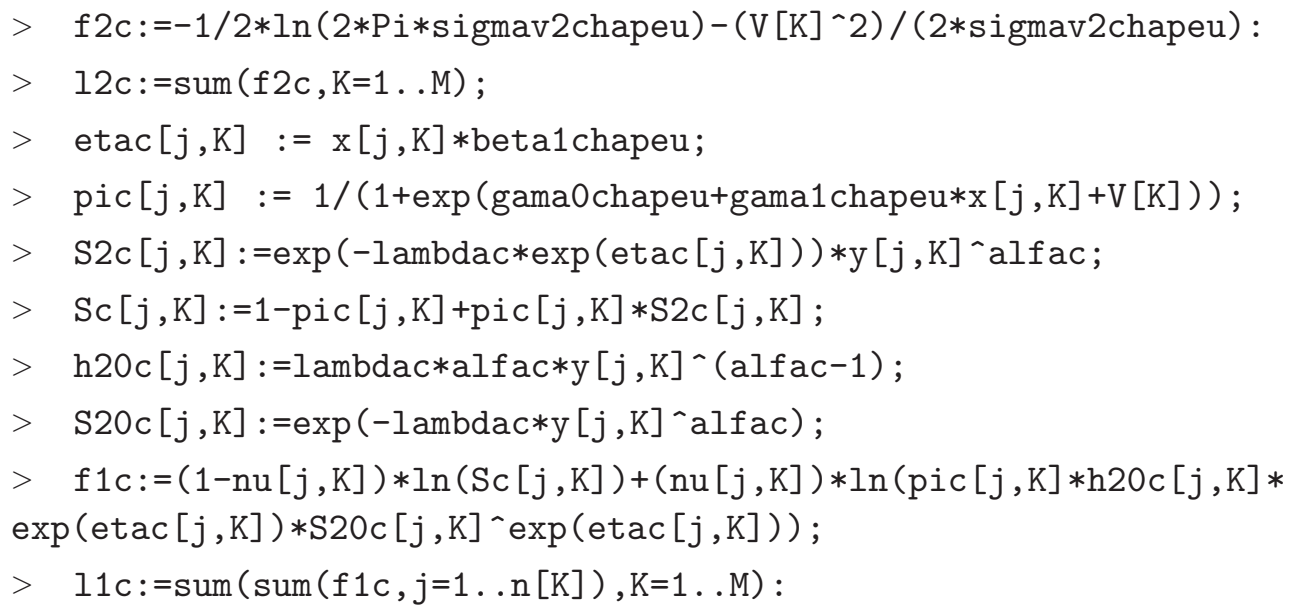

Aqui abaixo está a definição da log-verossimilhança completa 
$>1 \mathrm{c}:=11 \mathrm{c}+12 \mathrm{c}:$

$>1 c c:=\operatorname{subs}(V[1]=\operatorname{phi}[5,2], \operatorname{subs}(\mathrm{V}[2]=\operatorname{phi}[6,2], \operatorname{subs}(\mathrm{V}[3]=\operatorname{phi}[7,2]$, $\operatorname{subs}(\mathrm{V}[4]=\operatorname{phi}[8,2], \operatorname{subs}(\mathrm{V}[5]=\operatorname{phi}[9,2], \operatorname{subs}(\mathrm{V}[6]=\operatorname{phi}[10,2]$, $\operatorname{subs}($ sigmav2 $=$ sigmav2chapeu, 1c)))))) ):

Aqui tem que fazer o novo loop, para estimar alfa e lambda

$>\operatorname{phi2:=matrix}(2,2,[10, a l f a, 10,1$ ambda $])$;

Definir vetor de derivadas

$>\mathrm{Uc}:=\operatorname{matrix}(2,1,[\operatorname{diff}(1 \mathrm{cc}, \mathrm{alfac}), \operatorname{diff}(1 \mathrm{cc}, \mathrm{lambdac})])$ :

$>\mathrm{U} 2 \mathrm{c}:=\operatorname{matrix}(2,1)$ :

Definindo a matriz hessiana

$>$ Hc := hessian (lcc, [alfac, lambdac]):

$>\mathrm{H} 2 \mathrm{c}:=$ matrix $(2,2)$;

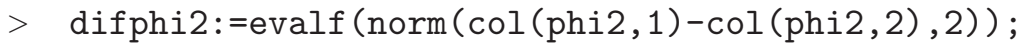

$>$ passo2 :=0.1;

$>$ for t from 1 to 1000 while (difphi2 > 0.001) do

for a from 1 to 2 do

phi2 [a, 1] := phi2 [a, 2]:

end do;

alfac: $=\operatorname{phi} 2[1,1]$;

lambdac: $=\operatorname{phi} 2[2,1]$;

for a from 1 to 2 do

$\mathrm{U} 2 \mathrm{c}[\mathrm{a}, 1]:=\operatorname{evalf}(\operatorname{subs}(\{\operatorname{alfac}=\operatorname{phi} 2[1,1], \operatorname{lambdac}=\operatorname{phi} 2[2,1]\}, \mathrm{Uc}[\mathrm{a}, 1]))$ :

end do:

for a from 1 to 2 do

for $b$ from 1 to 2 do

$\mathrm{H} 2 \mathrm{c}[\mathrm{a}, \mathrm{b}]:=\operatorname{evalf}(\operatorname{subs}(\{\mathrm{alfac}=\operatorname{phi} 2[1,1], \operatorname{lambdac}=\operatorname{phi} 2[2,1]\}, \mathrm{Hc}[\mathrm{a}, \mathrm{b}]))$ :

end do:

end do:

for a from 1 to 2 do

phi2 $[\mathrm{a}, 2]:=\operatorname{phi2}[\mathrm{a}, 1]-\operatorname{passo} 2 *($ evalm(inverse(H2c) \&* (U2c)) $[\mathrm{a}, 1])$;

end do;

phi2 $[1,2]:=$ 'if' $(\operatorname{phi} 2[1,2]<=0,0.01$, phi2 $[1,2])$;

phi2 $[2,2]:=$ 'if' $(\operatorname{phi} 2[2,2]<=0,0.01$, phi2 $[2,2])$;

\# atualiza a condição de parada para a convergência

difphi2:=evalf (norm $(\operatorname{col}(\operatorname{phi} 2,1)-\operatorname{col}(\operatorname{phi} 2,2), 2))$ :

print $(" t=", t)$;

end do; 
$>$ alfachapeu:=phi2 [1,2]; lambdachapeu:=phi2 [2,2];

Aqui estão as estimativas finais

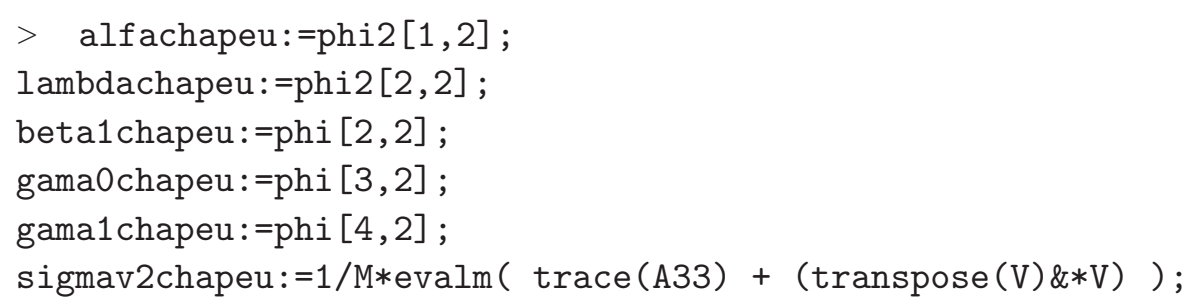




\section{Referências Bibliográficas}

[1] Berger, J. O. (1985). Statistical decision theory and bayesian analysis. New York: Springer Verlag.

[2] Berkson, J.; Gage, R. (1952). Survival curve for cancer patients following treatment, Journal of the American Statistical Association 47:501-515.

[3] Cancho, V. G.; Rodrigues, J.; Castro, M. (2008). A bayesian model for survival data with a cure rate. Manuscrito a ser submetido.

[4] Castro, M.; Cancho, V. G.; Rodrigues, J. (2007). A flexible model for survival data with a surviving fraction. Manuscrito a ser submetido para Statistical Modelling.

[5] Chen, M.-H.; Ibrahim, J. G.; Sinha, D. (1999). A new bayesian model for survival data with a surviving fraction, Journal of the American Statistical Association 94:909-919.

[6] Chen, M.-H.; Ibrahim, J. G.; Sinha, D. (2002). Bayesian inference for multivariate survival data with a surviving fraction, Journal of Multivariate Analysis 80:101-126.

[7] Chib, S.; Greenberg, E. (1995). Understanding the Metropolis-Hastings algorithm, The American Statistician; 49(4): 327-335.

[8] Cowless, M. K.; Carlin, B. P. (1996). Markov Chain Monte Carlo convergence diagnostics: a comparative review, Journal of the American Statistical Association 91(434):883-904.

[9] Cox, D. R. (1972). Regression models and life tables (with discussion), Journal of Royal Statistical Society, Ser. B 34:187-220.

[10] Dempster, A.; Laird, N.; Rubin, D. (1977). Maximum likelihood from incomplete data via EM algorithm, Journal of the Royal Statistical Society, Ser. B 39: 1-38.

[11] Efron, B. (1979). Bootstrap methods: another look at the jackknife, Annals of Statistics 7: 1-26. 
[12] Efron, B. (1982). The jackknife, the bootstrap and other resampling plans. Philadelphia: SIAM.

[13] Feller, W. (1967). An introduction to probability theory and its applications, v.1, 3.ed. New York: Wiley.

[14] Gelman, A.; Rubin, D. R. (1992). Inference from iterative simulation using multiple sequences. Statistical Science, 7(4):457-511.

[15] Geman, S.; Geman, D. (1984). Stochastic relaxation, Gibbs distributions and the bayesian restoration of images. IEEE Transactions on pattern analysis and machine intelligence, $\mathbf{6}$, 721-741.

[16] Henderson, C. R. (1975). Best linear unbiased estimation and prediction under a selection model, Biometrics 31:423-447.

[17] Ibrahim, J. G.; Chen, M.-H.; Sinha, D. (2001) Bayesian survival analysis. New York: Springer Verlag.

[18] Kalbfleisch, J. D.; Prentice R. L. (1980). The statistical analysis of failure data. New York: Wiley.

[19] Louis, T. (1982). Finding the observed information matrix when using the EM algorithm. Journal of the Royal Statistical Society Series B, 44: 226-233.

[20] Maller, R. A.; Zhou, X. (1996). Survival analysis with long-term survivors. New York: Wiley.

[21] Maplesoft. (2005). Maple User Manual. Maplesoft.

[22] McGilchrist, C. A. (1994). Estimation in generalized mixed models, Journal of the Royal Statistical Society Series B 56:61-69.

[23] McGilchrist, C. A. e Yau, K. K. W. (1995). The derivation of BLUP, ML, REML estimation methods for generalised linear mixed models, Communications in Statistics - Theory and Methods 24(12):2963-2980.

[24] McLachan, G. J. e Krishnan, T. (1997). The EM algorithm and extensions. New York: Wiley.

[25] Mizoi, M. F. (2004). Influência local em modelos de sobrevivência com fração de cura. Tese de Doutorado - IME - USP. São Paulo. 
[26] Rodrigues, J.; Louzada-Neto, F. (2006). On the unification of the long-term survival models. Manuscrito submetido para Statistics and Probability Letters.

[27] Spiegelhalter, D.; Thomas, A.; Best, N.; Lunn, D. (2007). Winbugs user manual. Emperial College and MRC, UK.

[28] Tanner, M.; Wong, W. (1987). The calculation of posterior distributions by data augmentation. Journal of the American Statistical Association, 82: 528-550.

[29] Thompson, R. (1980). Maximum likelihood estimation of variance components. Math. Operationsforsch. Statist., Ser. Statistics, 11: 545-561.

[30] Venables, W. N.; Smith, D. M., R Development Core Team (2008). An introduction to R. Disponível em ¡http://cran.r-project.org/doc/manuals/R-intro.pdf ¿. Acesso em 23 maio 2008.

[31] Yau, K. K. W.; Ng, A. S. K. (2001). Long-term survivor mixture model with random effects: application to a multi-centre clinical trial of carcinoma, Statistics in Medicine 20: 1591-1607.

[32] Yin, G.; Ibrahim, J. G. (2005). Cure rate models: a unified approach, The Canadian Journal of Statistics 33(4): 559-570. 